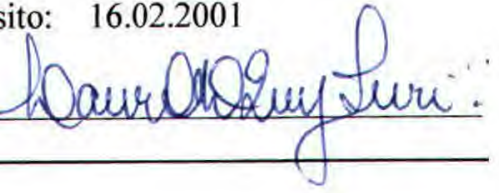

\title{
Métodos Computacionais na Teoria de Curvas Algebróides Irredutíveis
}

\author{
Marcelo Escudeiro Hernandes
}

Orientador: Prof. Dr. Abramo Hefez

Tese apresentada ao Instituto de Ciências Matemáticas e de Computação - ICMC-USP, como parte dos requisitos para obtenção do título de Doutor em Ciências - Matemática.

USP - São Carlos

Fevereiro de 2001

2001 Mathematics Subject Classification. 13P10, 13F25, 14B05,14H20, 14H50, 14Q05, 32S05, 32S10, $32 \mathrm{~S} 15$. Palavras chaves: base Standard, Curvas Algebróides Irredutiveis, Semigrupo de Valores, Anel Local, Módulo de Diferenciais, Equisingularidade, Equidiferenciabilidade, Classificação de Curvas.

E-mail:mehernandes@uem.br 


\section{A Comissão Julgadora:}

Prof. Dr. Abramo Hefez

Prof. Dr. Arnaldo Leite Pinto Garcia

Prof. Dr. Paulo Roberto Brumatti

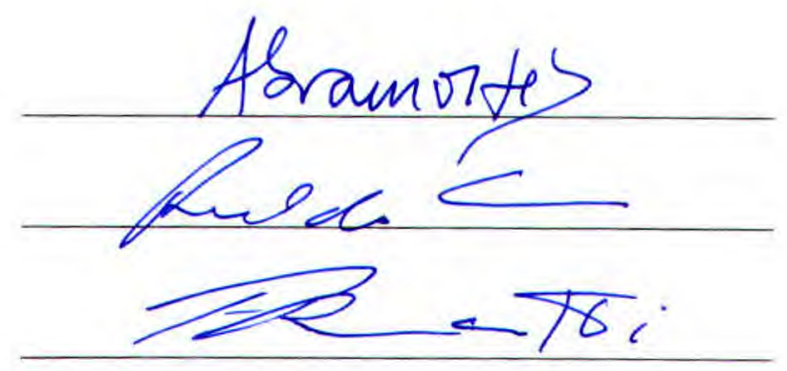

Profa. Dra. Maria Aparecida Soares Ruas

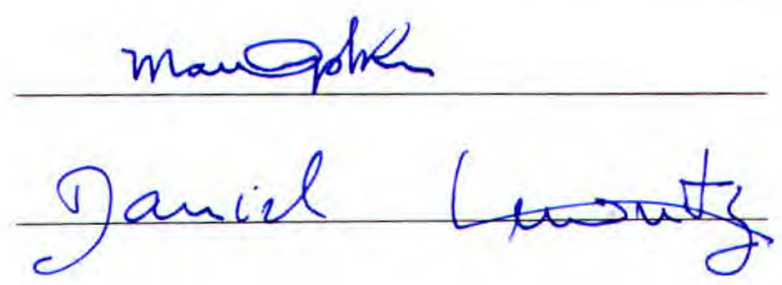

Prof. Dr. Daniel Levcovitz 


\section{Agradecimentos}

À Deus, pelo dom da vida e pela oportunidade de fazer o que gosto.

Ao Prof. Dr. Abramo Hefez pela orientação e pela amizade consolidada nestes últimos anos.

Aos meus pais, Cláudio e Izabel, minha irmã Sheila e a pequena Gabriela, que percorreram comigo mais esta caminhada.

À Maria Elenice Rodrigues por todo o seu carinlıo e apoio.

Aos vellos e novos amigos, pelo companherismo.

Ao DMA-UEM, ICMC-USP e pós-graduação em Matemática da UFF, por propiciarem as condições ideais para a execução deste trabalho.

A CAPES, pelo financiamento parcial deste trabalho. 


\section{Abstract}

In this work we will present algorithms that, allow to compute. Staudard Bases for local rings and module of differentials associated to algebroid irreducible curves.

Several applications are given, as for example, the computation of the semigroup values and the set of orders of differentials of a given curve, some criteria for elimination of parameters and the classification, modulo isomorphism between local rings, of all irreducible algebroid plane curves up to multiplicity 4. 


\section{Resumo}

Neste trabalho apresentamos algoritmos que permitem computar Bases Standard de anéis locais e módulos de diferenciais associados a curvas algebróides irredutíveis.

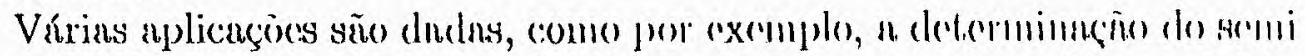
grupo e o do conjunto das ordens de diferenciais de uma curva, alguns critérios de eliminação de parâmetros e a classificação, a menos de isomorfismo entre anéis locais, de todas as curvas algebróides irredutíveis planas até a multiplicidade 4 . 


\section{Índice}

Introdução $\quad$ xiii

1 Base Standard 1

1.1 Ordens Monomiais . . . . . . . . . . . . . . . . . 1

1.2 Base Standard para Subálgebras . . . . . . . . . . . . 3

1.3 Base Standard para Submódulos . . . . . . . . . . . 17

2 Equidiferenciabilidade $\quad 25$

2.1 Semigrupo de Valores de uma Curva . . . . . . . . . . . . 25

2.1.1 Semigrupos de Curvas Planas ... . . . . . . . 35

2.2 O Módulo de Diferenciais de uma Curva . . . . . . . . . . 42

2.2.1 Curvas de Interseção Completa . . . . . . . . 51

3 Equivalência de Curvas $\quad 59$

3.1 Invariantes Aritméticos . . . . . . . . . . . . . . 59

3.2 Classificação de Curvas . . . . . . . . . . . . . . 63

4 Eliminação de Parâmetros $\quad 89$

5 Ramos de Gênero 1 103

5.1 Base Standard e Lacunas Especiais . . . . . . . . . . . . . 103

5.2 Ramos com Invariante de Zariski Fixo . . . . . . . . . . . . 109

5.3 Ramo $\lambda$-canônico . . . . . . . . . . . . . . . . 121

5.4 Diagramas Admissíveis . . . . . . . . . . . . . 131

6 Ramos de Multiplicidade Baixa 141

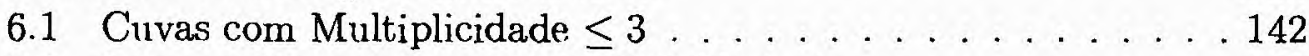

6.2 Lacunas Especiais para Multiplicidade 4 . . . . . . . . 143

6.3 Formas Normais para Multiplicidade 4 . . . . . . . . . 146 
xii

6.4 Classificação das Curvas de Multiplicidade 4 . . . . . . . . 150

6.5 Ramos com Multiplicidade 5 . . . . . . . . . . . . . 161

6.6 Tabelas . . . . . . . . . . . . . . . 166

6.7 Resposta à uma questão de Zariski . . . . . . . . . . 168

A Semigrupos do tipo $\left\langle 2 n_{1}, 2 m_{1}, v_{2}\right\rangle \quad 181$

$\begin{array}{ll}\text { Bibliografia } & 197\end{array}$

$\begin{array}{ll}\text { Símbolos e Notações } & 201\end{array}$

$\begin{array}{ll}\text { Indice Remissivo } & 203\end{array}$ 


\section{Introdução}

O estudo local de uma singularidade isolada se mostra ainda hoje um campo vasto, com questões relevantes e que permanecem sem respostas.

Tomemos o caso mais simples, curvas analíticas planas irredutíveis, on seja, séries analíticas irredutíiveis de $\mathbb{C}[X, Y]]$ módulo a relação de associado. Uma questão natural que surge é reconhecer quando duas tais curvas são topologicamente equivalentes, isto é, dadas duas séries analíticas $f, g \in$ $\mathbb{C}[X, Y]]$, como decidir se existem vizinhanças abertas $U$ e $V$ da origern de $\mathbb{C}^{\mathbb{E}}$ e um homeomomorfismo $\Phi: U \rightarrow V$ tais que $\Phi\left(U \cap f^{-1}(0)\right)=V \cap g^{-1}(0)$.

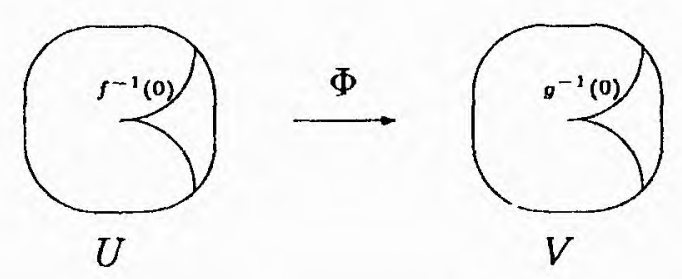

Tal questão foi completamente respondida por K. Brauner, W. Burau e $\mathrm{O}$. Zariski, que utilizando ferramentas algébricas e topológicas, mostraram que dois germes de curvas analíticas irredutíveis planas são topologicamente equivalentes se, e somente se, os germes possuem os mesmos pares característicos de Puiseux, ou equivalentemente, o mesmo semigrupo de valores.

$\mathrm{O}$ conceito de equivalência topológica, traduzido em termos de igualdade de semigrupos de valores, que Zariski denominon de equisingularidade, se estende às curvas algebróides espaciais sobre corpos arbitrários.

A primeira pergunta que surge é: Como determinar o scmigrupo de valores de uma dada curva algebróide irredutível?

Uma outra pergunta interessante é: Como decidir se dois germes de curvas analíticas irredutíveis planas equisingulares são analiticamente equiva- 
xiv

lentes; ou seja, como decidir se algum dos homeomorfismos $\Phi$ que realizam a equivalência topológica é um isomorfismo analítico?

Tal questão central da teoria de curvas irredutíveis planas ainda permanece abèrta.

$\mathrm{Na}$ falta de invariantes discretos completos para a equivalência analítica, corno é o caso do semigrupo para a equivalência topológica, uma possível estratégia para resolver o problema da classificação analítica é obter formas normais para as curvas, isto é, encontrar uma representação simples para as curvas de modo a reconhecer facilmente quando duas curvas são analiticamente equivalentes.

No entanto, vários obstáculos surgem. Por exemplo, o que é uma representação simples? Tal representação existe? Como obtê-la?

No caso de curvas algebróides irredutíveis planas (ramos) com multiplicidade baixa, encontramos na literatura respostas às questões acima e tabelas.com as referidas formas normais. Em, [E] (1965), Ebey classifica os ramos com multiplicidade menor que 4 e alguns de multiplicidade 4 . Bruce e Gaffuey, em [BGa] (1982), utilizando métodos da teoria de Mather obtém parte dos resultados de Ebey.

A exemplo da equivalência topológica, podemos estender a relação de equivalência analítica ao caso formal e às curvas espaciais em característica arbitráriar." Tal relação será denominada simplesmente de equivalência.

Em característica zero, vários invariantes com respeito à equivalência são conliecidos. Por exemplo, o invariante $\lambda$ de Zariski (no caso plano), o número $\tau$ de Tjurina (no caso de interseção completa) e o conjunto $\Lambda$ das ordens de diferenciais (curvas quaisquer).

Como computar tais invariantes?

O invariante $\lambda$ de Zariski, que é definido para curvas irredutíveis planas, é facilmente computado quando a curva é dada por uma parametrização de Puiseux (Veja [Z2]). Quando a curva tem gênero 1 e é definida por meio de uma série $f \in K[[X, Y]]$, Peraire em [Pe2], indica como computá-lo. Mas, e para curvas com gênero superior a 1 ?

O número $\tau$ de Tjurina de uma série $f \in K[[X, Y]]$, é talvez um dos invariantes mais estudados por estar relacionado a vários conceitos importantes, como por exemplo, a dimensão da base da deformação miniversal de $f$ e o comprimento do submódulo de torção do módulo de diferenciais da curva.

Quảndo temos uma curva específica dada por uma série $f \in K[[X, Y]]$, podemos computar $\tau$ através da codimensão do ideal Jacobiano (Veja [Z2]), 
mas e quando a curva se apresenta na forma paramétrica?

Vários trabalhos sobre o número $\tau$ de Tjurina merecem destaque. Por exemplo, Delorme em [D2], 1978, descreve um algoritmo para calcular o número de Tjurina mínimo para curvas com fixado semigrupo de gênero 1 , obtendo fórmulas explícitas. Briançon, Granger e Maisonobe, em [BGM], descrevem um algoritmo para computar o menor valor de $\tau$ para curvas com um semigrupo com os dois primeiros geradores fixados, estendendo assim, o resultado de Delorme. Peraire, em [Pe2], modifica tal algoritmo e computa o menor valor de $\tau$ para curvas com semigrupo de gêuero $1 \mathrm{e}$ invariante $\lambda$ de Zariski fixo. Em [Pe1] apresenta utm algoritmo para calcular $\tau$ mínino para um semigrupo fixado de gênero qualquer.

E quanto a $\Lambda$ ?

Este invariante permite obter os invariantes $\lambda$ e $\tau$ e portanto é mais fino do que os anteriores. Baseado nesse invariante, define-se a relação de equidiferenciabilidade que intermedia a equisingularidade $e$ a equivalência de curvas algebóides irredutíveis. Assim, podemos nos perguutar como decidir se duas curvas são equidiferenciáveis, ou seja, como computar $\Lambda$ ? Delorme em [D2] fornece um algoritmo para calcular $\Lambda$ no caso de curvas genéricas de gênero 1.

Algumas dessas qucstões serão respondidas neste traballıo, in!̣́meras permanecem e outras tantas podem ser formuladas, perpetuando assim, o estudo das curvas algebróides irredutíveis.

O objetivo central deste trabalho é o de apresentar métodos sistemáticos, que podem ser implementados, para o cálculo dos invariantes aritméticos descritos acima, unificar os resultados conhecidos que ellvolvem tais invarianl.cs sob o ponto de vista dos algoritmos introduzidos e estudar algumas aplicações dos mesmos ao problema da classificação de curvas planas irredutíveis.

Passemos à descrição dos capítulos.

O Capítulo 1 contém as ferramentas gerais básicas para o nosso estudo. Apresentamos uma generalização da teoria de SAGBI desenvolvida em [RS], para subálgebras e submódulos do anel das séries de potências formaịs. Tais objetos serão chamados de Bases Standard.

No Capítulo 2, aplicamos a teoria desenvolvida no Capítulo 1 para o caso de curvas algebróides irredutíveis. Na primeira seção, obtẹmos um algoritmo que permite computar uma Base Standard para o ancl local $\mathcal{O}$ do numa curva algebróide irredutível qualquer, e apartir dessa Base obtemos o semigrupo $\Gamma$ associado a curva em questão. Isto permite, entre outras coisas, decidir quando duas curvas dadas são equisiugulares. No caso de curvas planas em 
característica zero, otimizamos o algoritmo e o comparamos com os métodos conhecidos.

Na segunda seção, um outro algoritmo, que permite computar uma Base Standard para o módulo de diferenciais $\mathcal{O} d \mathcal{O}$, é apresentado. Apartir disso calculamos o conjunto $\Lambda$ para qualquer curva algebróide irredutível sobre $1 \mathrm{~m}$ corpo de característica zero. Isso possibilita, por exemplo, decidir se duas curvas são equidiferenciáveis ou não. As curvas de interseção completa mereceram um parágrafo próprio, pois para estas existe uma interessante relação entre o ideal jacobiano e o módulo de diferenciais, mais especificamente entre suas Bases Standard Mínimas.

$\mathrm{O}$ Capítulo 3 se restinge às curvas algebróides planas (ramos). Relacionamos o problema da classificação de ramos com isomorfismos entre. Anéis de Puiseux. Apresentamos um exemplo de como os métodos desenvolvidos, permitem computar todos os possíveis conjuntos $\Lambda$ para uma particular classe de equisingularidade. Introduzimos ainda o diagrama de lacunas especiais, que é também um invariante com respeito à relação de equivalência de ramos e otimizamos o algoritmo para computar parte das diferenciais não exatas minimais.

Ò Capítulo 4 contém generalizações dos critérios de eliminação de parâmetros contidos em [E] e [Z2].

No Capítulo 5, nos restringimos ao estudo dos ramos de gênero 1. Além de.reinterpretar resultados conhecidos utilizando os algoritmos do Capítulo 2, caracterizamos todos os posșíveis diagramas de lacunas especiais, 'ou seja, todos os conjuntos $\Lambda$ que podem ocorrer para curvas de gênero 1 .

No Capítulo 6 realizamos a classificação dos ramos para todas as classes de equisingularidades dadas por semigrupos de multiplicidade menor que 5 e para algumas de multiplicidade 5, o que estende a tabela até então conlhecida dada por Ebey em $[\mathrm{E}]$. Respondemos também a uma pergunta formulada por Zariski em [Z2] e para ist,o, realizamos a classificação das classes de equisingularidades dadas pelos semigrupos gerados por 6 e 7 e por 7 e 8 .

Incluimos ainda um apêndice no qual estudamos os ramos com semigrupos considerados em [LuP], caracterizando todos os possíveis conjuntos $\Lambda$ em função de certas lacunas. 


\section{Capítulo 1}

\section{Base Standard}

A teoria de Base de Groebner e o algoritmo de Buchberger para determinar um conjundo de geradores privilegiados para ideais em anéis de polinômios e séries de potências foi estendida, com sucesso, para outras situações. Isto permite aplicar a teoria ao estudo das singularidades de curvas algebróides irredutíveis, como mostraremos no próximo capítulo.

Em [RS], Robbiano e Sweedler, introduzem o conceito de Subălgebra Analog to Groebner Bases for Ideals (SAGBI). Nesse trabalho, são apresentados algoritmos para obter uma SAGBI para subálgebras finitamente geradas de um anel de polinômio sobre 1 m corpo, sıjeitas a determinàdas condições. O objetivo deste capítulo é estender tais resultados para o caso do anel de séries de potências, a fim de obtermos algoritmos para gerar bases especiais para algumas subálgebras e submódulos finitamente gerados no anel de séries de potências sobre um corpo $K$, tais bases serão por nós chamadas de Base Standard.

\subsection{Ordens Monomiais}

Denotemos por $K[[\underline{X}]]=K\left[\left[X_{1}, \ldots, X_{n}\right]\right]$ o anel das séries de potências em $X_{1}, \ldots, X_{n}$, com coeficientes no corpo $K$. O monômio $\prod_{i=1}^{n} X_{i}^{\alpha_{1}}$. será representado por $\underline{X}^{\alpha}$ onde $\alpha=\left(\alpha_{1}, \ldots, \alpha_{n}\right)$. Note que $1=X_{1}^{0} \ldots \ldots X_{n}^{0}$ é $\mathrm{um}$ monômio.

O conjunto de todos os monômios será denotado por TI.

Definição 1.1 Uma ordem monomial sobre $\mathrm{T \Gamma}$ é uma ordem total $\preceq$, tal que: 
- $1 \preceq t$ para todo $t \in \mathbb{T}$.

- Se $t_{1} \preceq t_{2}$, então $t t_{1} \preceq t t_{2}$, para todo $t, t_{1}, t_{2} \in \mathbb{T}$.

Uma propriedade importante de uma ordem monomial sobre Tr, é que com essa ordem, TT é bem ordenado. Isso é consequiência do lema abaixo e do fato de que se $t_{1} \mid t_{2}$, então $t_{1} \preceq t_{2}$ para todo $t_{1}, t_{2} \in \mathrm{T}$.

Lema 1.1 (Lema de Dickson) Todo subconjunto, não vazio, $C \subseteq \mathrm{T}$ possui um subconjunto finito $R \subseteq C$, tal que para todo $c \in C$, existe $r \in R$ com $r \mid c$.

Dem.: Seja $C=\left\{t_{1}, t_{2}, \ldots\right\} \subseteq \mathbb{T}$ e $I=\langle C\rangle$ o ideal gerado por $C$ em $K[[\underline{X}]]$. Como $K[[\underline{X}]]$ é noetheriano, a seqüência ascendente de ideais

$$
I_{1}=\left\langle t_{1}\right\rangle \subset I_{2}=\left\langle t_{1}, t_{2}\right\rangle \subset \cdots
$$

é estacionária, i.e., $I=\left\langle t_{1}, t_{2}, \ldots, t_{k}\right\rangle$ para algum $k$.

Uma vez que todo $t \in C$ pode ser escrito como

$$
t=\sum_{i=1}^{k} f_{i} t_{i},
$$

com $f_{i} \in K[[\underline{X}]]$, temos que $t=p_{i} t_{i}$ para algum $1 \leq i \leq k$ e algum $p_{i} \in \mathbb{T}$. Assim, para todo elemento do conjunto $C$, existe um elemento de $\left\{t_{1}, t_{2}, \ldots, t_{k}\right\} \subseteq C$ que o divide.

Dentre as ordens monomiais mais comuns sobre $\mathbb{T}$, destacamos:

1. Ordem Lexicográfica $\left(\leq_{L e x}\right)$ : Define-se $\underline{X}^{\alpha} \leq_{\text {Lex }} \underline{X}^{\beta}$ se, e somente se, $\alpha=\beta$ ou a primeira coordenada não mula, a partir da esquerda, se existir, de $\beta-\alpha$ é positiva.

2. Ordem Lexicográfica Graduada $\left(\leq_{G r L e x}\right)$ : Dizemos que $\underline{X}^{\alpha} \leq_{G r L e x} \underline{X}^{\beta}$ se, e somente se:

a) $\sum_{i=1}^{n} \alpha_{i}<\sum_{i=1}^{n} \beta_{i}$, onde $<$ é a ordern usual em $\mathbb{N}$, ou

b) $\sum_{i=1}^{n} \alpha_{i}=\sum_{i=1}^{n} \beta_{i}$ e $\underline{X}^{\alpha} \leq_{L e x} \underline{X}^{\beta}$.

Definimos o grau de um monômio $\underline{X}^{\alpha}$, como sendo $\operatorname{deg}\left(X^{\alpha}\right)=\sum_{i=1}^{n} \alpha_{i}$. 
3. Ordem Pesada $\left(\leq_{\rho}\right)$ : Sejam $\rho=\left(\rho_{1}, \ldots, \rho_{n}\right) \in \mathbb{N}^{n},<$ a ordem usual de $\mathbb{N}$ e $\preceq$ uma ordem monomial sobre $\mathbb{T}$, denotaremos por $\alpha$. $\beta$ o produto escalar usual de $\alpha$ e $\beta$.

Dizemos que $\underline{X}^{\alpha} \leq_{\rho} \underline{X}^{\beta}$ se, e somente se, uma das possibilidades abaixo ocorre:

a) $\rho . \alpha<\rho . \beta$, ou

b) $\rho . \alpha=\rho . \beta$ e $\underline{X}^{\alpha} \preceq \underline{X}^{\beta}$.

Chamamos $\rho$ de peso e $\leq_{\rho}$ de ordem pesada com respeito ao peso $\rho$ e a ordem monomial $\preceq$. Definimos o grau pesado relativo à $\rho$, como sendo $\operatorname{deg}_{\rho}\left(\underline{X}^{\alpha}\right)=\rho . \alpha$.

Note que se $\rho=(0, \ldots, 0)$, então $\leq_{\rho}$ coincide com $\preceq$ e ainda que, se $\preceq$ é a ordem lexicográfica e $\rho=(1, \ldots, 1)$, cntão $\leq_{\rho}$ é a ordem lexicográfica graduada.

Seja $f=\sum_{\alpha \in A} a_{\alpha} \underline{X}^{\alpha} \in K[[\underline{X}]]$, denotamos por $\mathbb{T}(f)$, o conjunto dos monômios de $f$, i.e., $\left\{\underline{X}^{\alpha} ; a_{\alpha} \neq 0\right\}$.

Definimos a potência líder de $f$ com respeito a uma dada ordem monomial, como sendo o menor elemento $l p(f)$ de $\mathbb{T}(f)$.

Seja $G \subseteq K[[X]]$, denotaremos por $l p(G)$, o conjunto de todas as potências líderes dos elementos de $G$, ou seja, $l p(G)=\left\{l p(g) ; g \in G^{*}\right\}$, onde $G^{*}=G \backslash\{0\}$.

A partir deste ponto, fixamos uma ordem monomial $\preceq$. Assim, ao mencionarmos menor ou maior elemento, potência lídler, etc., estaremos nos referindo à ordem monomial fixada.

Indicaremos, por $l c(f)$ o coeficiente da potência líder na expressão de $f$ e por $l t(f)=l c(f) l p(f)$ o termo líder de $f$.

\subsection{Base Standard para Subálgebras}

Nesta seção, estenderemos os resultados de Robbiano e Sweedler para anéis de séries de potências.

Denotaremos por $\mathcal{M}_{\underline{X}}$, o ideal maximal de $K[[\underline{X}]]$.

Considere um elemento de $K[[\underline{X}]]^{*}$ escrito sob a forma

$$
\sum_{i \in I} a_{i} f_{i}
$$


com $a_{i} \in K^{*}$ e $f_{i} \in K[[\underline{X}]]$, definimos a altura de $\sum_{i \in I} a_{i} f_{i}$, como sendo

$$
h t\left(\sum_{i \in I} a_{i} f_{i}\right)=\min _{i \in I}\left\{l p\left(f_{i}\right)\right\} .
$$

Note que esta definição depende da representação $\sum_{i \in I} a_{i} f_{i}$, e não do elemento $\sum_{i \in I} a_{i} f_{i}$ em si. De fato, tem-se que $h t\left(\sum_{i \in I} a_{i} f_{i}\right) \preceq l p\left(\sum_{i \in I} a_{i} f_{i}\right)$.

Dizemos que $f_{j}$ contribui para a altura de $\sum_{i \in I} a_{i} f_{i}$, se

$$
l p\left(f_{j}\right)=\min _{i \in I}\left\{l p\left(f_{i}\right)\right\} .
$$

A amplitude de $\sum_{i \in I} a_{i} f_{i}$ é definida como sendo o número de termos $f_{j}$ em $\sum_{i \in I} a_{i} f_{i}$ que contribuem para a altura.

Seja $F=\left\{f_{1}, \ldots, f_{m}\right\} \subset \mathcal{M}_{\underline{X}}^{*}$. Considere o homomorfismo substil.uição

$$
\begin{array}{ccc}
\mathcal{S}_{\left(f_{1}, \ldots, f_{m}\right)}: K\left[\left[Y_{1}, \ldots, Y_{m}\right]\right] & \longrightarrow & K[[\underline{X}]] . \\
g & \mapsto g\left(f_{1}, \ldots, f_{m}\right)
\end{array}
$$

Um $F$-produto de potências é um elemento da forma

$$
F^{\alpha}=\mathcal{S}_{\left(f_{1}, \ldots, f_{m}\right)}\left(\prod_{i=1}^{m} Y_{i}^{\alpha_{i}}\right)=\prod_{i=1}^{m} f_{i}^{\alpha_{i}}
$$

onde $\alpha=\left(\alpha_{i}, \ldots, \alpha_{m}\right)$.

Definimos a $K$-subálgebra $K[[F]]$ de $K[[\underline{X}]]$ como sendo

$$
K[[F]]=\mathcal{S}_{\left(f_{1}, \ldots, f_{m}\right)}\left(K\left[\left[Y_{1}, \ldots, Y_{m}\right]\right]\right)
$$

Note que se $a \in K$, então $a=a F^{0}=a \prod_{i=1}^{m} f_{i}^{0}$.

Sejam $f \in K[[\underline{X}]]$ e $F \subset \mathcal{M}_{\underline{X}}^{*}$. Dizemos que $f$ se reduz a $g$ módulo $F$, escrevendo

$$
f \stackrel{F}{\longrightarrow} g
$$

se, existem um $F$-produto de potências $F^{\alpha}$ e $a \in K$, tais que

$$
g=f-a F^{\alpha},
$$

com $g=0$ ou $l p(f) \prec l p(g)$.

Escreveremos

$$
f \stackrel{F_{+}}{\longrightarrow} g
$$


quando existir uma cadeia, eventualmente infinita, de reduções módulo $F$, iniciando em $f$ e finalizando em $g$, i.e., $g$ não se reduz módulo $F$. Diremos, neste caso, que $g$ é uma redução final de $f$ módulo $F$.

Uma redução final $g$ de $f$, por um conjunto finito $F$, como acima, será chamada de redução completa, se para todo $t \in \mathbb{T}(g), t$ não se reduz módulo $F$.

Observação 1.1 1. Um processo de redução corresponde, de certo modo, ao processo de divisão pelos elementos de F. Note que, podemos eventualmente reduzir um elemento de várias maneiras distintas. Por exemplo, se $f=Y^{12}$ e $F=\left\{f_{1}=Y^{4}, f_{2}=Y^{3}+\sum_{i=4}^{\infty} X^{i}\right\}$, onde, a ordem monomial considerada é a ordem lexicográfica graduada, podemos obter duas reduções distintas de $f$, a saber, $g_{1}=f-f_{1}^{3}=0$ e $g_{2}=f-f_{2}^{4}$. Por este motivo é interessante indicar por qual $F$-produto de potências efetuamos a redução. Assim, escrevemos

$$
f \stackrel{f_{1}^{3}}{\longrightarrow} g_{1} \quad f \stackrel{f_{2}^{4}}{\longrightarrow} g_{2} .
$$

2. Note que se $f \stackrel{F_{+}}{\longrightarrow} g$, então $f-g \in K[[F]]$.

3. Como estamos manipulando séries de potências, o processo de redução pode eventualmente não finalizar em um número finito de passos. Admitiremos que de algum modo sempre possamos obter uma redução final e uma redução completa de um elemento de $K[[\underline{X}]]$ m.ódulo um subconjunto finito $F \subset \mathcal{M}_{\underline{X}}^{*}$.

4. Note que é possivel reduzir $f \in K[[\underline{X}]]=K\left[\left[X_{1}, \ldots, X_{n}\right]\right]$ módulo $F=\left\{f_{1}, \ldots, f_{m}\right\} \subset \mathcal{M}_{\underline{X}}^{*}$ se, e somente se, $l p(f)=\underline{X}^{\alpha}$ pertence ao semigrupo multiplicativo gerado por $l p(F)=\left\{l p\left(f_{i}\right)=\underline{X}^{\alpha_{i}} ; f_{i} \in F\right\}$, ou equivalentemente se $\alpha$ pertence ao semigrupo aditivo $\Gamma$ gerado por $\left\{\alpha_{i} ; i=1, \ldots, n\right\}$. Em particular, se $\mathbb{N}^{n} \backslash \Gamma$ é finito, então é possível. decidir, em um número finito de passos, se uma redução final de $f$ módulo $F$ é zero.

Definição 1.2 Dizemos que $F=\left\{f_{1}, \ldots, f_{m}\right\} \subset \mathcal{M}_{\underline{X}}^{*}$ é uma Base Standard de álgebras, se para todo $f \in K[[F]]^{*}$

$$
l p(f)=\operatorname{lp}\left(F^{\alpha}\right)
$$


para algum $\alpha \in \mathbb{N}^{m}$.

Uma Base Standard para uma subálgebra $A \subseteq K[[\underline{X}]]$ é uma Base St.andard de álgebras $F$, tal que $A=K[[F]]$.

No que 'segue, nesta seção, ıma Base Standard será sempre do álgebras e o semigrupo gerado por $v_{0}, \ldots, v_{m}$, será denotado por $\left\langle v_{0}, \ldots, v_{m}\right\rangle$.

Com essa notação, podemos reescrever a definição anterior do seguinte modo:

Um conjunto finito $F \subset \mathcal{M}_{\underline{X}}^{*}$ é uma Base Standard de álgebras se, e somente se,

$$
\langle l p(F)\rangle=l p(K[[F]]) .
$$

Note que, se existir uma Base Standard $F$ para uma subálgebra $A$, temos necessariamente $A=K[[F]]$ e que $l p(A)$ é um semigrupo multiplicativo finitamente gerado por $l p(F)$. Portanto, nem toda subálgebra de $K[[\underline{X}]]$ possui uma Base Standard.

Exemplo 1.1 Seja $F=\left\{X^{2}, \sum_{i=3}^{\infty} X^{i}\right\}$. O conjunto $F$ é uma Base Standard. De fato, se $f \in K[[F]]^{*}$, ent.ão $l p(f)=1$ ou $l p(f)=X^{\alpha}$, com $\alpha \geq 2$. Portanto $l p(f) \in\left\langle X^{2}, X^{3}\right\rangle=\langle l p(F)\rangle$.

Exemplo 1.2 Seja $F=\left\{X^{4}, X^{6}+X^{7}\right\}$. O conjunto $F$ não é uma Base. Standard. De fato, $2 X^{13}+X^{14}=\left(X^{6}+X^{7}\right)^{2}-\left(X^{4}\right)^{3} \in K[[F]]$, mas $X^{13} \notin$ $\left\langle X^{4}, X^{6}\right\rangle=\langle l p(F)\rangle$.

Como observamos anteriormente, a redução final de um elemento de $K[[\underline{X}]]$ módulo um subconjunto finito $F \subset \mathcal{M}_{\underline{X}}^{*}$ pode não ser única. No entanto, se $F$ for uma Base Standard, então a redução completa é única, como mostra a próxima proposição.

Proposição 1.1 Seja $F \subset \mathcal{M}_{X}^{*}$ uma Base Standard. Se $r_{1}$ e $r_{2}$ são duas reduções completas de $f \in K \|[\underline{X}]]$, ent.ão $r_{1}=r_{2}$.

Dem.: Sejam $r_{1}=f-\sum_{\alpha \in I} a_{\alpha} F^{\alpha}$ e $r_{2}=f-\sum_{\beta \in J} b_{\beta} F^{\beta}$ duas reduções completas de $f$ módulo $F$, assim $r_{2}-r_{1} \in K[[F]]$. Suponha que $r_{2} \neq r_{1}$. Como $r_{2}-r_{1} \in K[[F]]^{*}$ e $F$ é Base Standard de $A$, existiria um elemento de $l p(F)$ que dividiria algum elemento de $\mathbb{T}\left(r_{1}\right) \cup \mathbb{T}\left(r_{2}\right)$, um absurdo.

A proposição a seguir nos fornece uma caracterização para uma Base Standard. 
Proposição 1.2 Sejam $F=\left\{f_{1}, \ldots, f_{m}\right\} \subset \mathcal{M}_{\underline{X}}^{*}$ e $\left.A=K \|[F]\right]$. São equivalentes:

1. F é uma Base Standard para álgebras.

2. Para todo $f \in A$, qualquer redução final de $f$ módulo $F$ é zero.

3. Para todo $f \in A$, existe um processo de redução, tal que a redução final de $f$ módulo $F$, é zero.

Dem.: 1) $\Rightarrow$ 2) Seja $f \in A$ e $g$ uma redução final de $f$ módulo $F$, tal que $g \neq 0$, como $g=f-\sum_{\alpha \in I} a_{\alpha} F^{\alpha} \in A$ e $F$ é uma Base Standard, existe $\alpha \in I$ tal que $l p(g)=l p\left(F^{\alpha}\right)$, mas desta forma $g$ se reduz módulo $F$, o que contradiz a hipótese de $g$ ser uma redução final de $f$ módulo $F$.

2) $\Rightarrow 3$ ) Óbvio.

3) $\Rightarrow$ 1) Seja $f \in A^{*}$, como existe uma redução final de $f$ módulo $F$ que é zero, podemos escrever $f=\sum_{\alpha \in I} a_{\alpha} F^{\alpha}$, com $l p(f)=l p\left(F^{\alpha}\right)$ para algum $\alpha \in I$, assim $l p(f) \in\left\langle l p\left(F^{\alpha}\right)\right\rangle$ e portanto, $F$ é Base Standard.

Observação 1.2 1. É óbvio que $F=\left\{X_{1}, \ldots, X_{n}\right\}$ é uma Base Standard de $K\left[\left[X_{1}, \ldots, X_{n}\right]\right]$ com respeito a qualquer ordem monomial.

2. $O$ item 3 da proposição anterior, afirma que todo $f \in A^{*}$, tem uma representação da forma

$$
f=\sum_{\alpha \in I} a_{\alpha} F^{\alpha} \quad a_{\alpha} \in K^{*}
$$

com amplitude 1. No entanto, tal representação não é necessariamente única.

De fato sejam $f=\sum_{i=1}^{\infty} X^{i} Y Z, g=\sum_{i=1}^{\infty} X^{i}$ e $h=Y Z$ elementos de $K[[X, Y, Z]]$. $O$ conjunto $F=\{f, g, h\}$ é uma Base Standard de álgebras, com respeito a ordem lexicográfica graduada; uma vez que todo elemento não nulo de $K[[F]]$ tem sua potência líder divisivel pela potência líder de um elemento de $F$. Um exemplo de que a representação mencionada não é única pode ser dado por:

$$
f=f^{1} g^{0} h^{0} \quad f=f^{0} g^{1} h^{1}
$$

ambas de amplitude 1. Mais ainda, este exemplo sugere que pode haver elementos que podem ser omitidos em uma Base Standard. 
A observação anterior sugere definirmos Base Standard Mínima.

Definição 1.3 Seja $F=\left\{f_{1}, \ldots, f_{m}\right\} \subset \mathcal{M}_{\underline{X}}^{*}$ uma Base Standard de álgebras, dizemos que $F$ é Mínima, se para todo $\bar{f}_{i} \in F$,

$$
\left.l p\left(f_{i}\right) \notin\left\langle l p\left(f_{1}\right), \ldots, l \widehat{p}_{i}\right), \ldots, l p\left(f_{m}\right)\right\rangle .
$$

Neste sentido, no item 2 da observação anterior, $F^{\prime}=\{g, h\}$ é uma Base Standard Mínima.

Lema 1.2 Seja $F=\left\{f_{1}, \ldots, f_{m}\right\} \subset \mathcal{M}_{\underline{X}}^{*}$ uma Base Standard. $\operatorname{Se} l p\left(f_{i}\right) \in$ $\left\langle\dot{l} p\left(f_{1}\right), \ldots, l \widehat{p\left(f_{i}\right)}, \ldots, l p\left(f_{m}\right)\right\rangle$, então $F^{\prime}=F \backslash\left\{f_{i}\right\}$ é Base Standard de álgebras e. $K\left[\left[F^{\prime}\right]\right]=K[[F]]$.

Dem.: É claro que $\left\langle l p\left(F^{\prime}\right)\right\rangle=\langle l p(F)\rangle=l p(K[[F]])$ e que $K\left[\left[F^{\prime}\right]\right] \subseteq K[[F]]$.

Assim $\left\langle l p\left(F^{\prime}\right)\right\rangle \subseteq l p\left(K\left[\left[F^{\prime}\right]\right]\right) \subseteq l p(K[[F]])=\left\langle l p\left(F^{\prime}\right)\right\rangle$, i.e., $F^{\prime}$ é Base Standard.

Agora, sejam $f \in K[[F]]$ e $r$ a redução completa de $f$ módulo $F^{\prime}$. Se $r \neq 0$, então $r \in K[[F]] \backslash K\left[\left[F^{\prime}\right]\right]$, mas desta forma $l p(r) \in\langle l p(F)\rangle=\left\langle l p\left(F^{\prime}\right)\right\rangle$, um absurdo. Portanto $f \in K\left[\left[F^{\prime}\right]\right]$.

Pelo lema anterior, para obtermos uma Base Standard Mínima para uma suibálgebra $A \subseteq K[[\underline{X}]]$, basta eliminarmos de uma Base Standard $F=$ $\left\{f_{1}, \ldots, f_{m}\right\}$ de $A$, todos os elementos $f_{i} \in F$, tais que

$$
l p(f) \in\left\langle l p\left(f_{1}\right), \ldots, l \underline{l\left(f_{i}\right)}, \ldots, l p\left(f_{m}\right)\right\rangle .
$$

Para obtermos um algoritmo para Bases Standard de álgebras, é necessário definir $S$-processo de um par de séries, neste contexto das álgebras.

Definição 1.4 Seja $F$ um subconjunto finito de $K[[\underline{X}]]$, um. $S$-processo de $F$ é definido por:

$$
a F^{\alpha}-b F^{\beta}
$$

com a propriedade

$$
l p\left(a F^{\alpha}-b F^{\beta}\right) \succ \min \left\{l p\left(F^{\alpha}\right), l p\left(F^{\beta}\right)\right\}\left(=h t\left(a F^{\alpha}-b F^{\beta}\right)\right),
$$

sempre que $a F^{\alpha}-b F^{\beta} \neq 0$. 
Exemplo 1.3 Seja $F=\left\{f=X, g=X Y^{3}, h=Y^{4}+Y^{5}, l=Y^{6}+Y^{7}\right\}$, considerando a ordem lexicográfica graduada, alguns $S$-processos de $F$ são:

$$
\begin{gathered}
f g-g f=0 \\
h^{3} f^{4}-g^{4}=3 X^{4} Y^{13}+3 X^{4} Y^{14}+X^{4} Y^{15} \\
l^{2} f^{4}-g^{4}=2 X^{4} Y^{13}+X^{4} Y^{14}
\end{gathered}
$$

O teorema abaixo, cuja demonstração é análoga a demonstração dada em [RS] (theorem 2.8) é de extrema relevância para obter um algoritmo para Base Standard de álgebras.

Teorema 1.1 Seja $F \subset \mathcal{M}_{\underline{X}}^{*}$ finito. Então $F$ é uma Base Standard de álgebras se, e somente se, todo $S$-processo de $F$, se reduz a zero módulo $F$ por algum processo de redução.

Dem.: $\Rightarrow$ )Seja $S$ um $S$-processo de $F$. Como $S \in K[[F]]$ e $F$ é Base Standard, segue do item 3) da Proposição 1.2, que $S$ possui uma redução final zero, por algum processo de redı̧̧ão módulo $F$.

$\Leftrightarrow)$ Seja $f \in K[[F]]^{*}$. De todas as possíveis representações

$$
\text { (*) } f=\sum_{\alpha \in I} a_{\alpha} F^{\alpha}
$$

com $a_{\alpha} \in K^{*}$, considere as que tem altura máxima $h\left(=l p\left(F^{\alpha}\right)\right.$ para algum $\alpha)$ e dentre essas, escolha uma com menor amplitude possível.

Vamos provar que $l p(f)=h$, mostrando assim que $l p(K[[F]]) \subseteq\langle l p(F)\rangle$ e portanto que $F$ é uma Base Standard de álgebras.

Sabemos que $l p(f) \succeq h$. Suponha por absurdo que $l p(f) \succ h$, i.e., a amplitude da soma $(*)$ é no mínimo 2 . Sem perda de generalidade, podemos supor que $h=l p\left(F^{\beta}\right)=l p\left(F^{\gamma}\right)$, onde $\beta, \gamma \in I$. Dessa forma, existe $b \in K^{*}$ tal que $S=a_{\beta} F^{\beta}-b a_{\gamma} F^{\gamma}$ é um $S$-processo de $F$. Por hipótese, $S \stackrel{F_{+}}{\longrightarrow} 0$ por algum processo de redução, e portanto, podemos escrever

$$
S=\sum_{\delta \in J} c_{\delta} F^{\delta} \quad c_{\delta} \in K^{*}
$$

com $l p(S) \succ h$ e amplitude 1.

Agora, escrevendo

$$
a_{\beta} F^{\beta}+a_{\gamma} F^{\gamma}=S+(b+1) a_{\gamma} F^{\gamma}
$$


temos que .

$$
f=\sum_{\alpha \in I} a_{\alpha} F^{\alpha}=S+(b+1) a_{\gamma} F^{\gamma}+\sum_{\alpha \in I \backslash\{\beta, \gamma\}} a_{\alpha} F^{\alpha} .
$$

Se $b \neq-1$, obtemos uma representação de $f$ com altura $h$ e amplitude menor do que a da representação (*), uma vez que $S$ não contribui para a altura. Absurdo!

Se $b=-1$, obtemos uma representação de maior altura ou menor amplitude do que as da representação $(*)$. Novamente um absurdo.

Observação 1.3 Observe que na demonstração do teorema anterior, bastaria garantir que todo $S$-processo $S=a F^{\beta}-b F^{\gamma}$ admita uma representação da forma $S=\sum_{\alpha \in I} c_{\alpha} F^{\alpha}, c_{\alpha} \in K^{*}$ com altura maior que $\ln \left(F^{\beta}\right)=\operatorname{lp}\left(F^{\gamma}\right)$.

Antes de prosseguirmos, vejamos mais de perto o conjunto de todos os $S$ prọcessos de um conjunto finito $F=\left\{f_{1}, \ldots, f_{m}\right\} \subset \mathcal{M}_{\underline{X}}^{*} \subset K\left[\left[X_{1}, \ldots, X_{n}\right]\right]$.

Denotaremos por $\operatorname{ord}_{X_{j}}\left(\underline{X}^{\alpha}\right)=\alpha_{j}$ a ordem de $\underline{X}^{\alpha}$ na indeterminada $X_{j}$.

Um $S$-processo $S=a F^{\alpha}-b F^{\beta}$, a menos de constantes, é determinado pelos vetores $\alpha, \beta \in \mathbb{N}^{m}$, soluções do sistema homegêneo de equações linearr diofantinas:

$$
\left\{\begin{array}{ccc}
\sum_{j=1}^{m} \alpha_{j} \operatorname{ord}_{X_{1}}\left(l p\left(f_{j}\right)\right) & = & \sum_{j=1}^{m} \beta_{j} \operatorname{ord}_{X_{1}}\left(l p\left(f_{j}\right)\right) \\
\sum_{j=1}^{m} \alpha_{j} \operatorname{ord}_{X_{2}}\left(l p\left(f_{j}\right)\right) & = & \sum_{j=1}^{m} \beta_{j} \operatorname{ord}_{X_{2}}\left(l p\left(f_{j}\right)\right) \\
\vdots & \vdots & \vdots \\
\sum_{j=1}^{m} \alpha_{j} \operatorname{ord}_{X_{n}}\left(l p\left(f_{j}\right)\right) & = & \sum_{j=1}^{m} \beta_{j} \operatorname{ord}_{X_{n}}\left(l p\left(f_{j}\right)\right) .
\end{array}\right.
$$

$\mathrm{O}$ conjunto de todas as soluções de (1.1) é um semigrupo aditivo de $\mathbb{N}^{2 m}$, gerado pelo conjunto $M$ de todas as soluções não nulas de (1.1) que são mínimas com respeito à ordem parcial

$$
(\alpha, \beta) \preceq(\gamma, \delta) \Leftrightarrow\left\{\begin{array}{l}
\alpha_{j} \leq \gamma_{j} \text { e } \beta_{j} \leq \delta_{j} \\
\text { para } j=1, \ldots, m .
\end{array}\right.
$$

Pelo Lema de Dickson (Lema 1.1), segue imediatamente, que o conjunto $M$ é finito.

No caso em que $K \cdot[[\underline{X}]]=K[[X]]$, o sistema (1.1) se reduz a uma equação linear diofantina homogênea, e nesse caso, Clausen e. Fortenbacher, om [CF], fornecem um algoritmo eficiente para obter o conjunto $M$. 
Exemplo 1.4 Seja $\left.F=\left\{X^{4}, X^{6}+X^{7}, 2 X^{13}+X^{14}\right\} \subset \mathbb{C}[X]\right]$, o sistema (1.1) se resume a equação $4 W_{1}+6 W_{2}+13 W_{3}-4 Z_{1}-6 Z_{2}-13 Z_{3}=0$. Usando o algoritmo acima citado, temos que o conjunto $M$ das soluçóes mínimas desta equação é:

$$
\begin{aligned}
M= & (1,0,0,1,0,0),(3,0,0,0,2,0),(13,0,0,0,0,4),(0,2,0,3,0,0), \\
& (0,1,0,0,1,0),(0,13,0,0,0,6),(0,0,4,13,0,0),(0,0,6,0,13,0), \\
& (0,0,1,0,0,1),(2,3,0,0,0,2),(8,0,0,0,1,2),(1,0,2,0,5,0), \\
& (0,0,2,2,3,0),(0,1,2,8,0,0),(0,5,0,1,0,2),(5,1,0,0,0,2), \\
& (1,8,0,0,0,4),(0,0,2,5,1,0),(0,0,4,1,8,0)\} .
\end{aligned}
$$

Note que, toda solução de (1.1) é obtida como soma de elementos de $M$. Denotamos por $S_{M}$ o conjunto dos $S$-processos minimos, obtidos pelas soluções mínimas de (1.1). Vejamos através de um exemplo, como o conjunto $S_{M}$ determina, todos os $S$-processos de $F$.

No contexto do Exemplo 1.4, considere o $S$-processo de $F$ dado por $S=$ $X^{4}\left(X^{6}+X^{7}\right)^{2}\left(2 X^{13}+X^{14}\right)^{6}-2^{6}\left(X^{4}\right)^{4}\left(X^{6}+X^{7}\right)^{13}$. Como $S$ é determinado por $(\alpha, \beta)=(1,2,6,4,13,0)$ e $(\alpha, \beta)=(0,2,0,3,0,0)+(0,0,6,0,13,0)+$ $(1,0,0,1,0,0)$, podemos escrever:

$$
S=\left[\left(X^{6}+X^{7}\right)^{2}-\left(X^{4}\right)^{3}\right] *\left[\left(2 X^{13}+X^{14}\right)^{6}-2^{6}\left(X^{6}+X^{7}\right)^{13}\right] *\left[X^{4}-X^{4}\right]
$$

onde $[a-b] *[c-d]=[a c-b d]$.

Temos o seguinte lema:

Lema 1.3 Seja $F \subset \mathcal{M}_{\underline{X}}^{*}$ um subconjunto finito. Se alguma redução final de todo $S$-processo mínimo de $F$ é zero, então todo $S$-processo não nulo $S=a F^{\alpha}-b F^{\beta}$ de $F$ pode ser representado como

$$
S=\sum_{\omega \in \Omega} d_{\omega} F^{\omega},
$$

com $d_{\omega} \in K^{*} e h t\left(a F^{\alpha}-b F^{\beta}\right) \prec h t\left(\sum_{\omega \in \Omega} d_{\omega} F^{\omega}\right)$.

Dem.: Seja $A=K[[F]]$ e $S_{M}$ o conjunto dos $S$-processos mínimos de $F$.

Podemos supor, sem perda de generalidade, que todo $S$-processo é da forma $F^{\alpha}-a F^{\beta}$, onde $a \in K$ é univocamente determinado.

Seja $S_{i}=F^{\alpha_{i}}-a_{i} F^{\beta_{i}} \in S_{M}, \operatorname{com} \alpha_{i}, \beta_{i} \in \mathbb{N}^{\sharp F}$. Como $S_{i} \stackrel{F_{+}}{\longrightarrow} 0$, por algum processo de redução, pelo item 2 da Observação 1.2 podemos escrever

$$
S_{i}=\sum_{\gamma_{i} \in \Gamma_{i}} b_{\gamma_{i}} F^{\gamma_{i}}
$$


com $b_{\gamma_{i}} \in K^{*}$ e amplitude 1, logo ht. $\left(\sum_{\gamma_{i} \in \Gamma_{i}} b_{\gamma_{i}} F^{\gamma_{i}}\right) \succ \operatorname{lp}\left(F^{\alpha_{i}}\right)=\operatorname{lp}\left(F^{\beta_{i}}\right)$. $\Lambda \operatorname{ssim} F^{\gamma_{i}}=a_{i} F^{\beta_{i}}+\sum_{\gamma_{i} \in \Gamma_{i}} b_{\gamma_{i}} F^{\gamma_{i}}$.

Seja $S=F^{\delta}-c F^{*}$ um $S$-processo não mulo de $F$, então

$$
\begin{gathered}
F^{\delta}=\prod_{i \in I}\left(F^{\alpha_{i}}\right)^{n_{i}} e \\
F^{\epsilon}=\prod_{i \in I}\left(F^{\beta_{i}}\right)^{n_{i}},
\end{gathered}
$$

$\operatorname{com} n_{i} \in \mathbb{N}$.

Assim,

$$
\begin{aligned}
F^{\delta} & =\prod_{i \in I}\left(F^{\alpha_{i}}\right)^{n_{i}}=\prod_{i \in I}\left(a_{i} F^{\beta_{i}}+\sum_{\gamma_{i} \in \Gamma_{i}} b_{\gamma_{i}} F^{\gamma_{i}}\right)^{n_{i}}= \\
& =\prod_{i \in I} a_{i}\left(F^{\beta_{i}}\right)^{n_{i}}+\sum_{\omega \in \Omega} d_{\omega} F^{\omega}=\prod_{i \in I} a_{i} F^{\epsilon}+\sum_{\omega \in \Omega} d_{\omega} F^{\omega},
\end{aligned}
$$

onde $h t\left(\sum_{\omega \in \Omega} d_{\omega} F^{\omega}\right) \succ h t\left(F^{\delta}-c F^{\epsilon}\right)$. Como a constante $c$ é univocamente determinada, temos que $\prod_{i \in I} a_{i}=c$, seguindo assim que

$$
S=\sum_{\omega \in \Omega} d_{\omega} F^{\omega} .
$$

Dester modo, do lema acima e da Obscrvação 1.3, podemos reescrever o Teorema 1.1 como segue.

Teorema 1.2 Seja $F \subset \mathcal{M}_{X}^{*}$ finito. Então $F$ é uma Base Standard para $K[[F]]$ se, e somente se, todo $S$-processo mínimo de $F$ se reduz a zero, por algum processo de redução.

Exemplo 1.5 Seja $F=\left\{X^{4}, X^{6}+X^{7}, 2 X^{13}+X^{14}\right\} \subset \mathbb{C}[[X]]$. Vimos no Exemplo 1.4 que os $S$-processos mínimos de $F$ são da forma $S=a F^{\alpha}-b F^{\beta}$ com.

$$
\begin{aligned}
(\alpha, \beta) \in\{ & (1,0,0,1,0,0),(3,0,0,0,2,0),(13,0,0,0,0,4),(0,2,0,3,0,0), \\
& (0,1,0,0,1,0),(0,13,0,0,0,6),(0,0,4,13,0,0),(0,0,6,0,13,0), \\
& (0,0,1,0,0,1),(2,3,0,0,0,2),(8,0,0,0,1,2),(1,0,2,0,5,0), \\
& (0,0,2,2,3,0),(0,1,2,8,0,0),(0,5,0,1,0,2),(5,1,0,0,0,2), \\
& (1,8,0,0,0,4),(0,0,2,5,1,0),(0,0,4,1,8,0)\}
\end{aligned}
$$


Note que o $1^{\circ}, 5^{\circ}$ e o 90 elementos deste conjunto, nos dão S-processos nulos, portanto não necessitam ser analisados.

$O 2^{\circ}$ e o o 4으, o $3^{\circ}$ e e o $7^{\circ}$, o $6^{\circ}$ e o o $8^{\circ}$, o $10^{\circ}$ e e o $13^{\circ}$, o $11^{\circ}$ e e o $14^{\circ}$, o $12^{\circ}$ e o 15o, o $16^{\circ}$ e o $18^{\circ}$, o $17^{\circ}$ e o $19^{\circ}$ nos dão, a menos de constantes, $o$ mesmo $S$-processo. Assim, basta analisar os $S$-processos mínimos dados por: $(3,0,0,0,2,0),(13,0,0,0,0,4),(0,13,0,0,0,6),(2,3,0,0,0,2),(8,0,0,0,1,2)$, $(1,0,2,0,5,0),(5,1,0,0,0,2)$ e. $(1,8,0,0,0,4)$, i.e..,

$$
\begin{gathered}
\left(X^{4}\right)^{3}-\left(X^{6}+X^{7}\right)^{2}=-\left(2 X^{13}+X^{14}\right) \stackrel{F_{+}}{\longrightarrow} 0 \\
16\left(X^{4}\right)^{13}-\left(2 X^{13}+X^{14}\right)^{4}=-32 X^{53}-24 X^{54}-8 X^{55}-X^{56} \\
64\left(X^{6}+X^{7}\right)^{13}-\left(2 X^{13}+X^{14}\right)^{6}=640 X^{79}+\cdots \\
4\left(X^{4}\right)^{2}\left(X^{6}+X^{7}\right)^{3}-\left(2 X^{13}+X^{14}\right)^{2}=8 X^{27}+11 X^{28}+4 X^{29} \\
4\left(X^{4}\right)^{8}-\left(X^{6}+X^{7}\right)\left(2 X^{13}+X^{14}\right)^{2}=-8 X^{33}-5 X^{34}-4 X^{35} \\
\left(X^{4}\right)\left(2 X^{13}+X^{14}\right)^{2}-4\left(X^{6}+X^{7}\right)^{5}=-16 X^{31}-39 X^{32}-40 X^{33}-20 X^{34}-4 X^{35} \\
4\left(X^{4}\right)^{5}\left(X^{6}+X^{7}\right)-\left(2 X^{13}+X^{14}\right)^{2}=-X^{28} \\
16\left(X^{4}\right)\left(X^{6}+X^{7}\right)^{8}-\left(2 X^{13}+X^{14}\right)^{4}=96 X^{53}+\cdots
\end{gathered}
$$

Como qualquer potência acima de 15 pode ser obtida usando produto de potências dos elementos de $F$, temos que todo $S$-processo mínimo possui uma redução final zero. Desta forma, $F$ é uma Base Standard, a saber uma Base Standard Minima, para a subálgebra $A=\mathbb{C}[[F]]$.

Fixada uma ordem monomial e uma subálgebra finitamente gerada $A$ de $K[[\underline{X}]]$, temos que $A$ admite uma Base Standard se, e somente se, o semigrupo (multiplicativo) das potências líderes de $A$, i.e., $l p(A)$ é finitamente gerado. É claro que se $F \subset A$ é tal que $l p(F)$ gera (como semigrupo) $l p(A)$, então $F$ é uma Base Standard para $A$.

Observação 1.4 Toda subálgebra $A \subseteq K[[X]]$ possui uma Base Standard. Basta verificar que $l p(A)$ é um semigrupo multiplicativo finitamente gerado, ou equivalentemente, que o semigrupo aditivo

$$
\Gamma=\left\{\alpha ; X^{\alpha}=l p(f) \text { para algum } f \in A\right\} \subseteq \mathbb{N}
$$

é finitamente gerado. 
Defina

$$
\begin{gathered}
v_{0}=\min \{\Gamma \backslash\{0\}\} \\
v_{1}=\min \left\{\Gamma \backslash\left\langle v_{0}\right\rangle\right\} \\
\vdots \\
v_{i}=\min \left\{\Gamma \backslash\left\langle v_{0}, \ldots, v_{i-1}\right\rangle\right\}
\end{gathered}
$$

onde $\left\langle v_{0}, \ldots, v_{i}\right\rangle$ é o semigrupo gerado por $\left\{v_{0}, \ldots, v_{i}\right\}$.

Agora note que existe $i<v_{0}$, tal que $\Gamma=\left\langle v_{0}, \ldots, v_{i}\right\rangle$. Isto segue dirrtamente do fato $v_{j}<v_{j+1}$ e $v_{j} \not \equiv v_{k} \bmod v_{0}$ para todo $j \neq k$.

O conjunto de geradores $\left\langle v_{0}, \ldots, v_{g}\right\rangle$, definido como ua olsservação antẹrior, é chamado de sistema mínimo de geradores de $\Gamma$, a saber, tal sistema está contido em qualquer outro sistema de geradores $\left\langle w_{0}, \ldots, w_{r}\right\rangle$.

De fato, como $v_{i}=\sum_{j=0}^{r} a_{j} w_{j}$ e $w_{j}=\sum_{k=0}^{g} b_{k j} v_{k}$, com $a_{j}, b_{k j} \in \mathbb{N}$ para todo $j=0, \ldots, r$, temos que

$$
v_{i}=\sum_{k=0}^{g} \sum_{j=0}^{r} a_{j} b_{k j} v_{k}
$$

assim existe um único $l \in\{0, \ldots, r\}$, tal que $a_{l}=b_{i l}=1$ e $a_{i j} b_{k j}=0$ para todo $k \neq i$ e todo $j=0, \ldots, r$. Em particular, tomando $j=l$, temos que $b_{k l}=0$ para todo $k \neq i$. Deste modo,

$$
w_{l}=\sum_{k=0}^{g} b_{k l} v_{k}=b_{i l} v_{i}=v_{i}
$$

i.e., $v_{i} \in\left\{w_{0}, \ldots, w_{r}\right\}$.

Assumiremos, a partir deste ponto, que dada uma subálgebra $A$, esta possua uma Base Standard com respeito a ordem monomial fixada.

Para efeito algorítmico, é interessante fixar uma maneira de efetuar o processo de redução (que estamos supondo sempre ser possível cliegar ao seu ponto final). Por exemplo, escolha a ordenação $\left(f_{1}, \ldots, f_{m}\right)$ para o conjunto $F=\left\{f_{1}, \ldots, f_{m}\right\} \subset \mathcal{M}_{\underline{X}}^{*}$ e se pudermos efetuar a redução de um elemento de $K[[\underline{X}]]$ por dois $F$-produtos $F^{\alpha}$ e $F^{\beta}$, escollemos efetuar a redução pelo $F$-produto de maior expoente $\alpha$, com respeito a ordem lexicográfica. 
Teorema 1.3 (Algoritmo) Seja $B \subset \mathcal{M}_{\underline{X}}^{*}$ finito e suponha que $\left.\operatorname{lp}(K \mid[B]]\right)$ seja um semigrupo multiplicativo finitamente gerado. Então, podemos obter uma Base Standard $F$ de $K[[B]]$ aplicando o algoritmo abaixo:

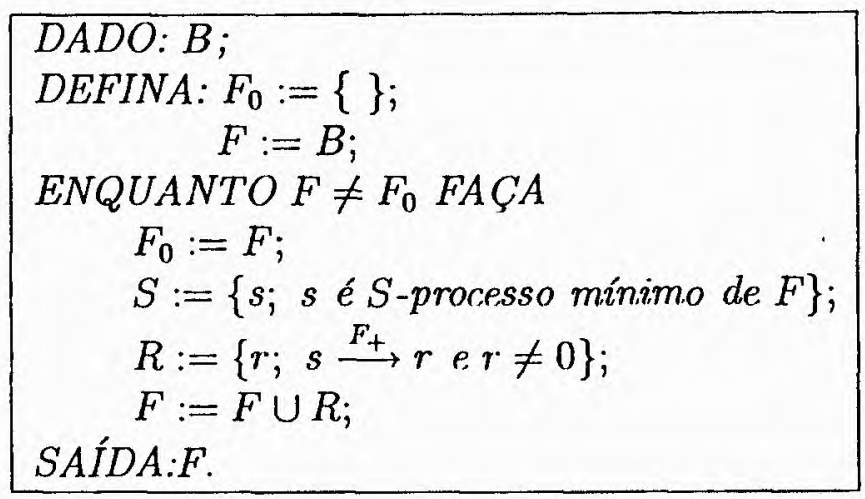

Dem.: Inicialmente fixemos uma maneira de efetuar o processo de redução. Veja que o conjunto $S$, assim como o conjunto $R$ são finitos para cada passo do algoritmo.

Supouha que $r_{i} \neq 0$ seja uma redução final de menor ordem de um $S$-processo mínimo em um dado passo $i$ do algoritmo acima. No passo $i+1$, além dos $S$-processos mínimos já considerados nos passos anteriores, que não nos trarão informações novas, introduzimos (eventualmente) $S$-processos mínimos cuja ordem é estritamente superior à ordem de $r_{i}$. Desta forma, como $l p(A)$ é um semigrupo finitamente gerado, a potência líder de uma redução final de menor ordem em cada passo $i$, não pode sulperar $m=\max \{l p(g) ; g$ pertence ao conjunto de geradores da Base Standard de $A$ \}. Portanto, existe um número finito de passos, tal que a partir deste, qualquer $S$-processo mínimo do conjunto $F$ possui uma redução final igual a zero, conseqüentemente, pelo Teorema 1.2, $F$ é uma Base Standard para $A$.

Exemplo 1.6 Seja $A \subset K[[X, Y]]$, a subálgebra gerada por $B=\left\{f_{1}=\right.$ $\left.X^{2}, f_{2}=\sum_{i=4}^{\infty} X^{i}, f_{3}=X^{7}, f_{4}=Y^{2}, f_{5}=Y^{3}+X^{8}\right\}$ e considere a ordem. lexicográfica graduada sobre $\mathrm{T}$.

Apliquemos o algoritmo do teorema anterior para o conjunto $B$.

Inicialmente note que todo monômio da forma $X^{\alpha} Y^{\beta}$ com $\alpha \in\langle 2,7\rangle$ e $\beta \in\langle 2,3\rangle$ pertence $a A$. Deste modo, todos os $S$-processos minimos de $F=B$ 
possuem uma redução final zero, exceto $S\left(f_{1}, f_{2}\right)=f_{2}-f_{1}^{2}=\sum_{i=5}^{\infty} X^{i}$, que coincide com sua redução final.

No próximo passo do algoritmo t.emos

$$
F=\left\{f_{1}=X^{2}, f_{2}=\sum_{i=4}^{\infty} X^{i}, f_{3}=X^{7}, f_{4}=Y^{2}, f_{5}=Y^{3}+X^{8}, f_{6}=\sum_{i=5}^{\infty} X^{i}\right\} .
$$

Uma simples verificação mostra que todo $S$-processo mínimo de $F$ se reduz a zero. Portanto F é uma Base Standard para $A$.

De acordo com o Lema 1.2, vemos ainda que $F_{0}=\left\{f_{1}, f_{4}, f_{5}, f_{6}\right\}$ é uma Base Standard Mínima para A. Uma vez que $X^{5}=f_{6}-f_{1}^{3}-f_{1} f_{6}$ e $Y^{3}=$ $f_{5}-f_{1}^{4}$, temos que $F_{1}=\left\{X^{2}, X^{5}, Y^{2}, Y^{3}\right\}$ é também uma Base Standard Mínima para $A$.

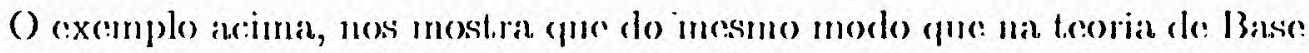
Standard de um ideal (Veja [Bec1] e [Bec2]), ıma Base Standard Mímima para subálgebra não ń nccessariamente úuica, no cuitanto, podemos definir Base Standard Reduzida, que é única, como mostramos a seguir.

Definição 1.5 Uma Base Standard Mínima $B$ é dita Reduzida se todo $f \in$ $B$ é mônico e $p \notin l p(K[[B]])$ para todo $p \in \mathbb{T}(f) \backslash\{l p(f)\}$.

Proposição 1.3 Seja uma subálgebra $A \subseteq K[[\underline{X}]]$. Se A possui uma Base Standard, então A possui uma única Base Standard Reduzida.

Dèm.: (Existência) Dada uma Base Standard, sempre podemos obter uma Base Standard Mínima usando o Lema 1.2. Assim, seja $F=\left\{f_{1}, \ldots, f_{m}\right\}$ uma Base Standard Mínima de $A$, podemos assumir, sem perda de generalidade, que todos os elementos de $F$ são mônicos.

Para cada $i=1, \ldots, m$, seja $r_{i}$ a redução completa de $f_{i}-\operatorname{lp}\left(f_{i}\right)$ módulo $F$. Então $F_{0}=\left\{l p\left(f_{1}\right)+r_{1}, \ldots, l p\left(f_{m}\right)+r_{m}\right\}$ é uma Base Standard Reduzida.

(Unicidade) Sejam $G=\left\{g_{1}, \ldots, g_{k}\right\}$ e $F=\left\{f_{1}, \ldots, f_{m}\right\}$ duas Bases Standard Reduzidas de $A$.

Tome $f_{i} \in F$ assim, existem $g_{j}, g_{l} \in G$ tais que $l p\left(g_{j}\right) \mid l p\left(f_{i}\right)$ e $l p\left(f_{i}\right) \mid$ $l p\left(g_{l}\right)$, como $l p\left(g_{j}\right) \chi l p\left(g_{l}\right)$ para $j \neq l$, segue que $l=j$ e $l p\left(f_{i}\right)=l p\left(g_{j}\right)$, em particular $m=k$, ou seja todas as Bases Standard Mínimas possuem o mesmo número de elementos.

- Podemos ordenar $G$ e $F$ de modo que $l p\left(f_{i}\right)=l p\left(g_{i}\right)$ para $i=1, \ldots, m$. Se existe $f_{i} \neq g_{i}$, então. $f_{i}-g_{i} \in A^{*}$, assim temos $p=\ln \left(f_{i}-g_{i}\right) \succ l p\left(f_{i}\right)=\ln p\left(g_{i}\right)$, onde $p \in \mathbb{I}\left(f_{i}\right) \cup \mathbb{T}\left(g_{i}\right)$. 
Como $f_{i}-g_{i} \in A^{*}, G$ e $F$ são-Bases Standard de $A$, pela Proposição 1.2 existem um $G$-produto de potências e um $F$-produto de potências, tais que $l p\left(G^{\alpha}\right)=l p\left(F^{\beta}\right)=p$, mas como $G$ e $F$ são Reduzidas isto não pode ocorrer, portanto $G=F$.

Observe que no exemplo anterior, $F_{1}$ é a Base Standard Reduzida da subálgebra $A$.

\subsection{Base Standard para Submódulos}

Sejam dados $A \subseteq K[[X]]$ uma subálgebra e $I$ um ideal de $A$. Uma questão natural que surge, é como obter uma Base Standard para o ideal $I$. Miller, em $[M]$, responde essa questão, no caso polinomial e denomina a Base Standard de $S G$-Base. Nesta seção adaptaremos o método de Miller para o anel das séries de potências e consideraremos a situação mais geral de um $A$ submódulo $M$ de $K[[\underline{X}]]$ finitamente gerado. É claro que se o conjunto $M$ for um subconjunto de $A$, então $M$ é um ideal de $A$ e estaremos no caso tratado por Miller, porém no contexto das séries de potências.

Deste ponto em diante, $A$ denotará uma subálgebra de $K[[\underline{X}]]$ com uma dada Base Standard $F$.

Definição 1.6 Sejam $A$ uma $K$-subálgebra de $K[[\underline{X}]]$ e $M$ um A-submódulo de $K[[\underline{X}]]$ finitamente gerado. Um subconjunto finito $G \subset \dot{M}$ é uma Base Standard de $A$-módulos para $M$, se para todo $m \in M^{*}$ tivermos

$$
l p(m)=l p(a g),
$$

com $a \in A$ e $g \in G$.

No que segue estaremos apenas considerando a ordem monomial que nos fornece a Base Standard $F$ de $A$.

Observação 1.5 Se $F \subset A$ é uma Base Standard de álgebras para A, então podemos escrever $a \in A^{*}$ como $a=\sum_{\alpha \in I} b_{\alpha} F^{\alpha}$, onde a soma tem amplitude 1 (Observação 1.2). Seja $F^{\alpha}$ o F-produto de potências de ordem mínima. Assim, $l p(a)=l p\left(F^{\alpha}\right)$ e portanto, podemos reescrever a definição acima do seguinte modo: 
Um subconjunto $G \subset M$ é uma Base Standard de módulos para $M$, se para todo $m \in M^{*}$

$$
l p(m)=l p\left(F^{\alpha} g\right)
$$

com $g \in G$ e algum $\alpha \in \mathbb{N}^{\sharp F}$.

Como no caso de Base Standard para ideais e para álgebras, podemos definir redução final módulo um subconjunto finito $G$ de $M$ no contexto de módulos.

Sejam $h \in K[[\underline{X}]]$ e $G=\left\{g_{1}, \ldots, g_{r}\right\} \subset M$. Dizemos que $h$ se reduz a $r$ módulo $(G, F)$, escrevendo $h \stackrel{G_{F}}{\longrightarrow} r$, se

$$
r=h-\sum_{i=1}^{r}\left(\sum_{\alpha_{i} \in \Theta_{i}} b_{\alpha_{i}} F^{\alpha_{i}}\right) g_{i},
$$

$\operatorname{com} b_{\alpha_{i}} \in K$ e $r \in K[[\underline{X}]]$ de forma que $r=0$ ou $l p(h) \prec l p(r)$. Quando não for mais possível a redução de $r$ módulo $(G, F)$, chamamos $r$ de uma redução final de $h$ e indicamos $h \stackrel{G_{F+}}{\longrightarrow} r$.

Como antes, uma redução final $r$ será chamada de uma redução completa, se para todo $t \in \mathbb{T}(r), t$ não se reduz módulo $(G, F)$.

A proposição a seguir nos dá uma caracterização de Base Standard para $A$-módulos.

Proposição 1.4 Sejam $A \subseteq K[[X]]$ uma $K$-subálgebra com Base Standard $F, M$.um $A$-submódulo de $K[[\underline{X}]]$ e $G=\left\{g_{1}, \ldots, g_{r}\right\} \subset M$. As seguintes afirmações são equivalentes:

1. G'é uma Base Standard de A-módulos para $M$.

2. Para todo $m \in M$, qualquer redução final de $m$ módulo $(G, F)$ é zero.

3. Para todo $m \in M$, existe uma redução final nula de $m$ módulo $(G, F)$.

4. Todo $m \in M^{*}$ tem uma representação (não necessariamente única!) da forma

$$
m=\sum_{i=1}^{r}\left(\sum_{\alpha_{i} \in \Theta_{i}} b_{\alpha_{i}} F^{\alpha_{i}}\right) g_{i}
$$

com amplitude ${ }^{-1}$. 
Dem.: Análoga a demonstração da Proposição 1.2.

Definição 1.7 Sejam $F$ uma Base Standard para a subálgebra $A \subseteq$ $K[[\underline{X}]]=K\left[\left[X_{1}, \ldots, X_{n}\right]\right]$ e um subconjunto $H \subseteq K[[\underline{X}]]$. Um $S$-processo do $\operatorname{par}(g, h) \in H \times H$, no contexto de A-módulos, é definido como sendo

$$
S(g, h)=a F^{\alpha} g-b F^{\beta} h,
$$

onde $a, b \in K, \alpha, \beta \in \mathbb{N}^{\sharp F}$ e com a propriedade de que se $a F^{\alpha} g-b F^{\beta} h \neq 0$, então

$$
l p\left(a F^{\alpha} g-b F^{\beta} h\right) \succ \min \left\{l p\left(F^{\alpha} g\right), l p\left(F^{\beta} h\right)\right\}=h t\left(a F^{\alpha} g-b F^{\beta} h\right) .
$$

Note que se $F=\left\{f_{1}, \ldots, f_{s}\right\}$ e fixado um par $(g, h) \in H \times H$, então um $S$-processo $a F^{\alpha} g-b F^{\beta} h$ é determinado, a menos de multiplicação por constantes, pelo vetor $(\alpha, \beta) \in \mathbb{N}^{2 s}$ que é solução do sistema

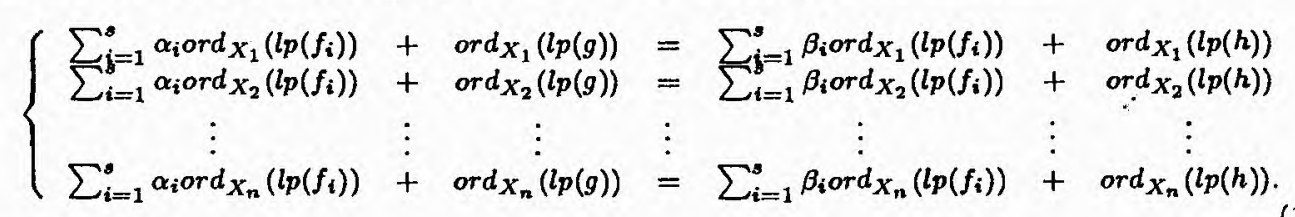

Apesar do conjunto das soluções do sistema (1.3) não ter estrutura de semigrupo quando $\operatorname{ord} d_{X_{j}}(g) \neq \operatorname{ord}_{X_{j}}(h)$ para algum $j=1, \ldots, n$, podemos definir o conjunto $N$ de todas as soluções (não nulas) que são mínimas com respeito à ordem parcial (1.2).

Além disso, pelo Lema de Dickson (Lema 1.1), temos que o conjunto $N$ é finito.

Uma solução de (1.3) é obtida através da adição de uma solução mínima pertencente a $N$ com uma solução do sistema homogêneo associado à (1.3), cujo conjunto de soluções sabemos ser finitamente gerado.

Como antes, toda solução de (1.3) nos dá um $S$-processo do par $(g, h)$. Os $S$-processos obtidos apartir dos elementos de $N$ serão chamados de $S$ processos mínimos de $(g, h)$, e o conjunto dos $S$-processo mínimos de $(g, h)$ será denotado por $S_{(g, h)}$. Os $S$-processos mínimos de todos os pares $(g, h) \in$ $H \times H$, serão chamados de $S$-processos mínimos de $H$.

O lema abaixo será útil para a apresentação de um algoritmo para uma Base Standard para módulos. 
Lema 1.4 Seja $A \subseteq K[[\underline{X}]]$ uma subálgebra com Base Standard $F$ e $M o$ A-módulo gerado por um subconjunto $G=\left\{g_{1}, \ldots, g_{r}\right\} \subset K[[\underline{X}]]$. Se uma redução final dos $S$-processos mínimos de $G$ é zero, então todo $S$-processo não nulo $S=a F^{\alpha} g-b F^{\beta} h$ com $(g, h) \in G \times G$ pode ser representado como

$$
S=\sum_{i=1}^{r}\left(\sum_{\omega_{i} \in \Omega_{i}} l_{\omega_{i}} F^{\omega_{i}}\right) g_{i},
$$

com. $l_{\omega_{i}} \in K \operatorname{eht}\left(\sum_{i=1}^{r}\left(\sum_{\omega_{i} \in \Omega_{i}} l_{\omega_{i}} F^{\omega_{i}}\right) g_{i}\right) \succ l p\left(F^{\alpha} g\right)=l p\left(F^{\beta} h\right)$.

Dem.: Podemos assumir, sem perda de generalidade que um $S$-processo do $\operatorname{par}(g, h) \in G \times G$ seja dado por $S=F^{\alpha} g-a F^{\beta} h$, onde $a \in K$ é univocamente determinado.

Seja $S_{i} \in S_{(g, h)}$. Como, $S_{i} \stackrel{G_{F+}}{\longrightarrow} 0$ pela Proposição 1.4, temos

$$
S_{i}=F^{\alpha_{i}} g-a_{i} F^{\beta_{i}} h=\sum_{i=1}^{r}\left(\sum_{\tau_{i} \in \Xi_{i}} b_{\tau_{i}} F^{\tau_{i}}\right) g_{i},
$$

onde $b_{\tau_{i}} \in K^{*}$ e $h t\left(\sum_{i=1}^{r}\left(\sum_{\tau_{i} \in \Xi_{i}} b_{\tau_{i}} F^{\tau_{i}}\right) g_{i}\right) \succ l p\left(F^{\alpha_{i}} g\right)=l p\left(F^{\beta_{i}} h\right)$. Donde segue o resultado para os $S$-processos mínimos.

Tome um $S$-processo não nulo qualquer do par $(g, h)$, i.e., $S=F^{\delta} g-c F^{\epsilon} h$, com $c \in K^{*}$ e $\delta, \epsilon \in \mathbb{N}^{\sharp F}$, então:

$$
\begin{aligned}
& F^{\delta} g=\left(\prod_{i \in I}\left(F^{\mu_{i}}\right)^{n_{i}}\right) F^{\alpha_{i}} g \\
& F^{\epsilon} h=\left(\prod_{i \in I}\left(F^{\lambda_{i}}\right)^{n_{i}}\right) F^{\beta_{i}} h
\end{aligned}
$$

com $n_{i} \in \mathbb{N},\left(\alpha_{i}, \beta_{i}\right)$ solução mínima do sistema (1.3) e $\left(\mu_{i}, \lambda_{i}\right)$ solução mínima do sistema homogêneo associado à (1.3).

Note que existe $d \in K^{*}$, tal que $\left(\prod_{i \in I}\left(F^{\mu_{i}}\right)^{n_{i}}\right)-d\left(\prod_{i \in I}\left(F^{\lambda_{i}}\right)^{n_{i}}\right)$ é um $S$-processo de $F$, assim usando o Teorema 1.1 , podemos escrever

$$
\prod_{i \in I}\left(F^{\mu_{i}}\right)^{n_{i}}=d \prod_{i \in I}\left(F^{\lambda_{i}}\right)^{n_{i}}+\sum_{\rho \in P} e_{\rho} F^{\rho}
$$

$\operatorname{com} e_{\rho} \in K^{*} \mathrm{e} \cdot h t\left(\sum_{\rho \in P} e_{\rho} F^{\rho}\right) \succ l p\left(\prod_{i \in I}\left(F^{\mu_{i}}\right)^{n_{i}}\right)=l p\left(\prod_{i \in I}\left(F^{\lambda_{i}}\right)^{n_{i}}\right)$.

Desse modo, 


$$
\begin{aligned}
F^{\delta} g & =\left(d \prod_{i \in I}\left(F^{\lambda_{i}}\right)^{n_{i}}+\sum_{\dot{\rho} \in P} e_{\rho}^{\rho} F^{\rho}\right) F^{\alpha_{i}} g= \\
& =\left(d \prod_{i \in I}\left(F^{\lambda_{i}}\right)^{n_{i}}+\sum_{\rho \in P} e_{\rho} F^{\rho}\right)\left(a_{i} F^{\beta_{i}} h+\sum_{i=1}^{r}\left(\sum_{\tau_{i} \in \Xi_{i}} b_{\tau_{i}} F^{\tau_{i}}\right) g_{i}\right)= \\
& =a_{i} d\left(\prod_{i \in I}\left(F^{\lambda_{i}}\right)^{n_{i}} F^{\beta_{2}} h\right)+\sum_{i=1}^{r}\left(\sum_{\omega_{i} \in \Omega_{i}} l_{\omega_{i}} F^{\omega_{i}}\right) g_{i}= \\
& =a_{i} d F^{\epsilon} h+\sum_{i=1}^{r}\left(\sum_{\omega_{i} \in \Omega_{i}} l_{\omega_{i}} F^{\omega_{i}}\right) g_{i},
\end{aligned}
$$

onde $h t\left(\sum_{i=1}^{r}\left(\sum_{\omega_{i} \in \Omega_{i}} l_{\omega_{i}} F^{\omega_{i}}\right) g_{i}\right) \succ l p\left(F^{\epsilon} g\right)=l p\left(F^{\delta} h\right)$.

Como a constante $c$ é univocamente determinada, segue que $c=a_{i} d \mathrm{e}$ assim

$$
S=\sum_{i=1}^{r}\left(\sum_{\omega_{i} \in \Omega_{i}} l_{\omega_{i}} F^{\omega_{i}}\right) g_{i}
$$

Com o auxílio do lema anterior e analogamente ao Teorema 1.2, temos o seguinte resultado.

Teorema 1.4 Sejam $A \subseteq K[[\underline{X}]]$ uma subálgebra com Base Standard F e. $M$ um A-submódulo de $K[[\underline{X}]]$ gerado por um subconjunto finito $G \subset K[[X]]$. Então $G$ é uma Base Standard para $M$ se, e somente se, a redução final, módulo $(G, F)$, de todo $S$-processo mínimo de $G$ é zero.

Observação 1.6 1. Note que se tomarmos o par $(g, g) \in G \times G$, um $S$-processo mínimo não é necessariamente trivial, pois o sistema (1.3) será homogêneo e como já vimos, um sistema deste tipo não admite apenas soluções triviais como mínimas.

No entanto, um $S$-processo não trivial de $(g, g)$ é da forma $S=a F^{\alpha} g-$ $b F^{\beta} g=\left(a F^{\alpha}-b F^{\beta}\right) g$. Como $a F^{\alpha}-b F^{\beta} \in A$ e $F$ é Base Standard de $A$, temos que $a F^{\alpha}-b F^{\beta} \stackrel{F_{+}}{\longrightarrow} 0$. Desta forma, se reduzirmos $a F^{\alpha}-b F^{\beta}$ por $c_{\gamma} F^{\gamma}$, podemos reduzir $S$ por $c_{\gamma} F^{\gamma} g$, assim $S \stackrel{G_{F+}}{\longrightarrow} 0$.

2. Se A é uma subálgebra com Base Standard $F$, então um: A-módulo $M$ finitamente gerado tem Base Standard se, e somente se, $l p(M)$ é finitamente gerado sobre $l p(A)$, ou melhor, $l p(M)$ é finitamente gerado sobre o semigrupo $\langle l p(F)\rangle$. Isto significa que existem $g_{1}, \ldots, g_{r} \in M$, tais que, para todo $m \in M$, existem $\alpha \in \mathbb{N}^{\| F}$ e $i \in\{1, \ldots ; r\}$, de modo que $l p(m)=l p\left(F^{\alpha} g_{i}\right)$. 
No que segue, vamos considerar apenas $A$-submódulos $M$ de $K[[\underline{X}]]$, tais que $l p(M)$ seja finitamente gerado sobre $l p(A)$.

Teorema 1.5 (Algoritmo) Seja A uma subálgebra dr: K\|XX\| conn. Bass. Standard $F$ e seja $B \subset K[[\underline{X}]]$ finito. Se $M$ é o $A$-módulo gerado por $B$ $l p(M)$ é finitamente gerado sobre l $p(A)$, então $M$ tem uma Base Standard $G$ dada pelo seguinte algoritmo:

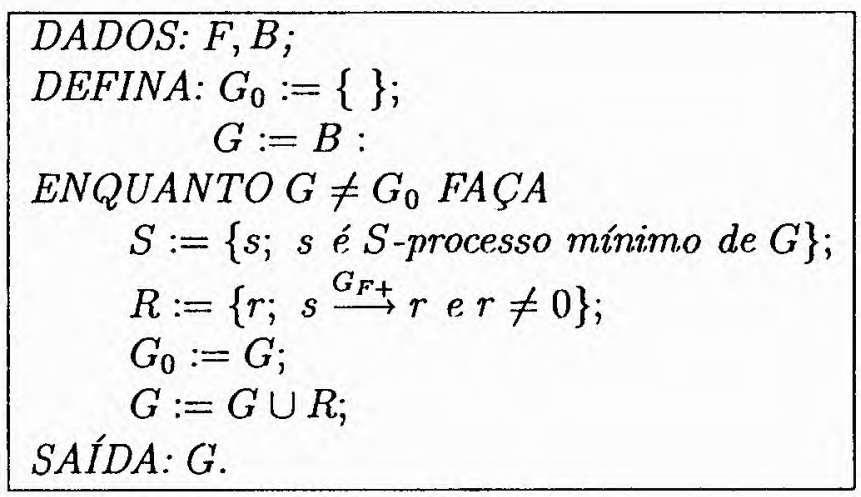

Dem.: Como $M$ admite Base Standard, existe $\max \{l p(g) ; g$ pertence à Base Standard de $M\}$, desta forma, usando o Teorema 1.4 o resultado segue como no Teorema 1.3 .

Da mesma forma que no caso de Base Standard para ideais e para subálgebras, podemos definir Base Standard Mínima e Base Standard Reduzida para módulos.

Definição 1.8 Sejam $M$ um A-módulo com Base Standard $G$ e F uma Base Standard da subálgebra $A \subseteq K[[\underline{X}]]$. A Base Standard $G$ é chamada de Base Standard Mínima, se para todo $h \in G$ tivermos $l p(h) \neq l p\left(F^{\alpha} g\right)$ para todo $g \in G \backslash\{h\}$, e todo $\alpha \in \mathbb{N}^{\sharp F}$.

Uma Base Standard Mínima $G$ de $M$ é dita Reduzida, se todo elemento $g \in G$ é mônico e $p \neq l\left(p\left(F^{\alpha} h\right)\right.$ para todo $h \in G$, todo $p \in \mathbb{T}(g) \backslash\{l p(g)\}$ e. qualquer $\alpha \in \mathbb{N}^{\sharp F}$.

Proposição 1.5 Seja $A \subseteq K[[\underline{X}]]$ uma subálgebra com Base Standard $F$. Se . $M$ é um A-submódulo de $K[[\underline{X}]]$ que possui Base Standard, então $M$ possui. uma única Base Standard Reduzida. 
Dem.: A existência, bem como a unicidade da Base Standard Reduzida segue como na Proposição 1.3.

Exemplo 1.7 Seja a subálgebra $\left.A=K \|\left[X^{4}, X^{9}+X^{15}\right]\right] \subset K[[X]]$, cuja Base. Standard Reduzida é $F=\left\{X^{4}, X^{9}+X^{15}\right\}$.

Se $a \in A$, então $\operatorname{ord}_{X}(a) \in\langle 4,9\rangle$, mais ainda, se $\left.\left.f \in K \| X\right]\right]$ é tal que $\operatorname{ord}_{X}(f) \geq 24$, então $f \in A$.

Considere o A-módulo $M$ gerado por $B=\left\{X^{3}, X^{8}-X^{14}\right\}$.

Note que, dado $f \in K[[X]]$ temos:

- Se ord $d_{X}(f) \geq 27$, então $l p(f)=\operatorname{lp}\left(X^{3}\right) l p(a), a \in A$.

- Se $\operatorname{ord}_{X}(f)=26$, então $l p(f)=l p\left(X^{8}-X^{14}\right) l p\left(\left(X^{9}+X^{15}\right)^{2}\right)$.

- Se ord $\operatorname{or}_{X}(f) \in\{23,24,25\}$, então $l p(f)=l p\left(X^{3}\right) \operatorname{lp}(a), a \in A$.

Assim, se $\operatorname{ord}_{X}(f) \geq 23$, então $f \in M$. Em particular, tem.os que $l p(M)$ é finitamente gerado sobre $l p(A)$, portanto $M$ possui Base Standard.

Apliquemos o algoritmo para $F=\left\{X^{4}, X^{9}+X^{15}\right\}$ e $B=\left\{X^{3}, X^{8}-X^{14}\right\}$.

\section{Passo 1}

T'emos que $S$ é a união dos conjuntos $S_{\left(X^{3}, X^{3}\right)}, S_{\left(X^{8}-X^{14}, X^{8}-X^{14}\right)} e$ $S_{\left(X^{3}, X^{8}-X^{14}\right)}$.

Pela Observação 1.6, vemos que os dois primeiros conjuntos nos dão $S$ processos cuja redução final é zero. No terceiro conjunto temos, a menos de constantes, dois $S$-processos, um deles é

$$
S_{1}=\left(X^{4}\right)^{8} X^{3}-\left(X^{9}+X^{15}\right)^{3}\left(X^{8}-X^{14}\right)
$$

com $\operatorname{ord}_{X}\left(S_{1}\right)>35$, que certamente terá uma redução final zero, módulo $(G, F)$.

$O$ segundo $S$-processo é

$$
S_{2}=\left(X^{9}+X^{15}\right) X^{3}-X^{4}\left(X^{8}-X^{14}\right)=2 X^{18}
$$

Se $\operatorname{car}(K)=2$, então o algoritmo finaliza.

Se car $(K) \neq 2$, então passamos ao próximo passo do algoritmo com $G:=$ $\left\{X^{3}, X^{8}-X^{14}, 2 X^{18}\right\}$. 


\section{Passo 2}

Além dos $S$-processos mínimos já computados no passo anterior, os quais terão redução zero neste passo, temos ainda $S_{\left(2 X^{18}, 2 X^{18}\right)}$, que não nos trará informações relevantes,

$$
\begin{aligned}
& S_{\left(X^{3}, 2 X^{18}\right)}=\left\{2\left(X^{4}\right)^{6} X^{3}-\left(X^{9}+X^{15}\right)\left(2 X^{18}\right),\right. \\
& \left.\left(X^{9}+X^{15}\right)^{3} X^{3}-\left(X^{4}\right)^{3}\left(2 X^{18}\right)\right\}, e \\
& S_{\left(X^{8}-X^{14}, 2 X^{18}\right)}=\left\{2\left(X^{4}\right)^{7}\left(X^{8}-X^{14}\right)-\left(X^{9}+X^{15}\right)^{2}\left(2 X^{18}\right)\right. \text {, } \\
& \left.2\left(X^{9}+X^{15}\right)^{2}\left(X^{8}-X^{14}\right)-\left(X^{4}\right)^{2}\left(2 X^{18}\right)\right\} \text {. }
\end{aligned}
$$

Uma vez que as alturas desses $S$-processos tem ordem superior a 23 , todos terão uma redução final zero módulo $(G, F)$, e portanto, o algoritmo finaliza.

Temos, assim, que uma Base Standard de $M$, na verdade Mínima, é dada. em qualquer característica por

$$
G:=\left\{X^{3}, X^{8}-X^{14}, 2 X^{18}\right\} .
$$




\section{Capítulo 2}

\section{Equidiferenciabilidade}

Neste capítulo aplicaremos a teoria desenvolvida no capítulo anterior para o estudo de uma curva algebróide irredutível $C$, veremos que importantes informações a respeito de $C$, tais como seu semigrupo de valores e valores de diferenciais não exatas, que até então pareciam difíceis de determinar, podem ser sistematicamente obtidos por meio dos algoritmos apresentados anteriormente.

No restante deste trabalho, $K$ é um corpo algebricamente fechado.

\subsection{Semigrupo de Valores de uma Curva}

Nesta seção, aplicaremos a teoria de Base Standard para subálgebras, de modo a obter o sistema mínimo de geradores do semigrupo de valores de uma curva algebróide irredutível.

Uma curva algebróide irredutivel $C$, em $K[[\underline{X}]]=K\left[\left[X_{1}, \ldots, X_{n}\right]\right]$, é um conjunto de equações $f_{1}, \ldots, f_{r}$ com $f_{i} \in \mathcal{M}_{\underline{X}}^{*} \subset K[[\underline{X}]]$ para $i=1, \ldots, r$, tal

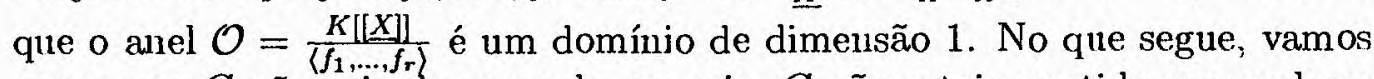
supor que $C$ não seja degenerada, ou seja, $C$ não esteja contida em nenhım hiperplano $X_{i}=0$ para algum $i=1, \ldots, n$.

$\mathrm{O}$ fecho inteiro $\overline{\mathcal{O}}$ no corpo de frações $\mathcal{K}$ de $\mathcal{O}$ é um anel de valorização discreta. Seja $t$ um parâmetro uniformizante de $\overline{\mathcal{O}}$, assim podemos considerar $\overline{\mathcal{O}}=K[[t]]$. Denotaremos por $\mathcal{M}$ o ideal maximal de $K[[t]]$ e por $x_{i}$, a classe residual de $X_{i}$ módulo o ideal $\left\langle f_{1}, \ldots, f_{r}\right\rangle$, i.e., $x_{i}=X_{i}+\left\langle f_{1}, \ldots, f_{r}\right\rangle$.

Dessa forma, temos $\mathcal{O}=K\left[\left[x_{1}, \ldots, x_{n}\right]\right] \subseteq \overline{\mathcal{O}}$ e representaremos $x_{i}$ em $\overline{\mathcal{O}}$ por $p_{i}(t)$, onde $p_{i}(t) \in \mathcal{M}^{*}$ para $i=1, \ldots, n$. 
Temos assim o monomorfismo de $K$-álgebras

$$
\begin{aligned}
\varphi: \mathcal{O} & \longrightarrow K[[t]] . \\
x_{i} & \mapsto p_{i}(t)
\end{aligned}
$$

A representação

$$
\left\{\begin{array}{ccc}
x_{1}= & p_{1}(t) \\
\vdots & \vdots & \vdots \\
x_{n} & = & p_{n}(t)
\end{array}\right.
$$

será chamada de paramètrização de $C$, onde identificamos $x_{i}$ com $\varphi\left(x_{i}\right)$. Claramente tem-se que $\varphi(\mathcal{O})=K\left[\left[p_{1}(t), \ldots, p_{n}(t)\right]\right]$.

Se $\operatorname{car}(K)=0$, então $C$ pode ser dada por uma parametrização de Puiseux, i.e., podemos, a menos de uma permutação dos $x_{i}$ 's, reparametrizar a curva $C$, de modo que $x_{1}=t^{v_{0}}$, onde $t^{v_{0}}=\min _{1 \leq i \leq n}\left\{l p\left(p_{i}(t)\right)\right\}$. Na verdade, é fácil verificar que, se $\operatorname{car}(K)$ não divide $v_{0}$, então $C$ admite uma parametrização de Puiseux.

O inteiro $v_{0}$, como acima definido, será chamado de multiplicidade de $C$ e denotado por mult $(C)$.

Anéis do tipo $\mathcal{A}=K\left[\left[t^{v_{0}}, p_{2}(t), \ldots, p_{n}(t)\right]\right]$, tais que o $\mathrm{MDC}$ de $v_{0}$ e dos expoentes dos termos das séries $p_{i}(t)$ 's é 1 , i.e., a parametrização $t^{v_{0}}, p_{2}(t)$, $p_{3}(t), \ldots, p_{n}(t)$ é primitiva, serão chamados de anéis de Puiseux.

Denotaremos por $v$ a valorização de $K[[t]]$, que obviamente é uma órdem monomial. Assim, apartir deste ponto, não mais mencionaremos a ordem monomial usada, uma vez que utilizaremos sempre a ordem monomial $v$.

Todo subanel $B$ de $K[[t]]$, determina um semigrupo $v(B) \subseteq \mathbb{N}$, em particular o semigrupo $v(\mathcal{O}):=v(\varphi(\mathcal{O}))$ é denominado de semigrupo associado a $C$.

Note que obter $\Gamma=v(\mathcal{O})$ não é trivial, uma vez que devemos calcular $v(g)$ para todo $g \in \mathcal{O}^{*}$. No entanto, como $\Gamma$ é finitamente gerado (Veja Observação 1.4), temos que $\mathcal{O}$, mais rigorosamente $\varphi(\mathcal{O})$, admite uma Base Standard em $K[[t]]$. A saber, as ordens dos elementos de uma Base Standard Mínima de $\mathcal{O}$, formam o sistema mínimo de geradores de $\Gamma$.

Como.vimos no.capítulo anterior (Proposição 1.3), todas as Bases Standard Mínimas de uma subálgebra possuem o mesmo número de elementos. No caso particular de curvas, esse número é o número de elementos do sistema mínimo de geradores de $\Gamma$, que será chamado de gênero do semigrupo $\Gamma$ ou gênero da curva $C$. 


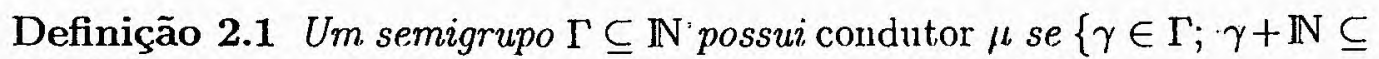
$\Gamma\} \neq\{\}$ e neste caso, define-se $\mu=\min \{\gamma \in \Gamma ; \gamma+\mathbb{N} \subseteq \Gamma\}$.

Uma importante propriedade do semigrupo $\Gamma$ de uma curva algebróide irredutível, é que este sempre possui condutor. De fato, tome $\frac{g}{h} \in \mathcal{K}$, com $g, h \in \mathcal{O}$, tais que $v\left(\frac{g}{h}\right)=1$. Logo $v(g)=v(h)+1$. É fácil verificar que para todo inteiro $i \geq(v(h)-1)(v(h)+1)$, temos $i \in\langle v(h), v(g)\rangle \subseteq \Gamma$.

$\mathrm{Na}$ verdade, o semigrupo associado a um anel de Puiseux $\mathcal{A}=$ $K\left[\left[t^{v_{0}}, p_{2}(t), \ldots, p_{n}(t)\right]\right]$ sempre possui condutor. Para verificar isto, é suficiente mostrar que existem elementos de $\mathcal{A}$, de modo que o MDC de suas ordens seja 1. Tais elementos podem ser obtidos aplicando o seguiute algoritmo:

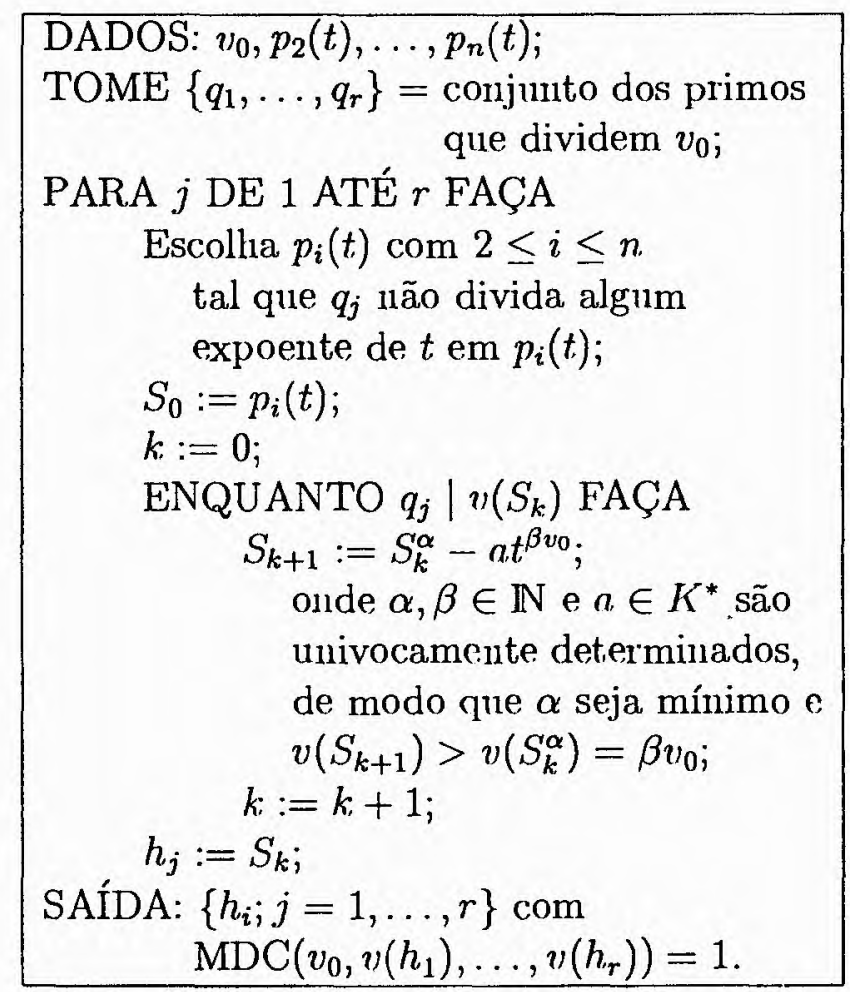

Note que a existência de $p_{i}(t)$, tal que $q_{j}$ seja coprimo com algum expoente de $t$ em $p_{i}(t)$, bem como a existência de $k<\infty$ tal que $q_{j} \backslash v\left(S_{k}\right)$, são garantidos pela primitividade da parametrização.

Uma vez que existem $h_{i} \in \mathcal{A}, \operatorname{com} w_{i}=v\left(h_{i}\right)$ para $i=1, \ldots, r$, tais que $\operatorname{MDC}\left(v_{0}, w_{1}, \ldots, w_{r}\right)=1$, podemos encontrar inteiros $\alpha_{i}$ para $i=0, \ldots, r$, de modo que $\sum_{i=0}^{r} \alpha_{i} w_{i}=1$. 
Denotando por $P=\left\{\alpha_{i} ; \alpha_{i} \geq 0\right\}$ e $N=\left\{\alpha_{i} ; \alpha_{i}<0\right\}$, podemos escrever

$$
v\left(\prod_{i \in P} h_{i}^{\alpha_{i}}\right)=1+v\left(\prod_{i \in N} h_{i}^{-\alpha_{i}}\right) .
$$

Como $\prod_{i \in P} h_{i}^{\alpha_{i}}, \prod_{i \in N} h_{i}^{-\alpha_{i}} \in \mathcal{A}$, o resultado segue como no caso do semigrupo de uma curva $C$, i.e., qualquer inteiro maior ou igual a

$$
\left(v\left(\prod_{i \in N} h_{i}^{-\alpha_{i}}\right)-1\right)\left(v\left(\prod_{i \in N} h_{i}^{-\alpha_{i}}\right)+1\right)
$$

pertence ao semigrupo $\left\langle v\left(\prod_{i \in N} h_{i}^{-\alpha_{i}}\right), v\left(\prod_{i \in P} h_{i}^{\alpha_{i}}\right)\right\rangle \subseteq v(\mathcal{A})$.

Observação 2.1 $O$ corpo de frações de um anel de Puiseux $\mathcal{A}$ é $K((t))$. Assim, seu fecho inteiro é $K[[t]]$.

De fato, seja $\mu$ o condutor de $v(\mathcal{A})$, isto implica que $t^{\mu}, t^{\mu+1} \in \mathcal{A}$, conseqüentemente $t$ pertence ao corpo de frações de $\mathcal{A}$, e a afirmação segue.

Os $S$-processos a serem calculados no Teorema 1.3 , são obtidos a partir das soluções mínimas de uma equação linear diofantina homogênea, como já observamos na capítulo anterior. Assim, faremos sempre uso do algoritmo de $[\mathrm{CF}]$ também citado no capítulo anterior.

As equações diofantinas, com as quais nos deparamos, são sempre da forma,

$$
\sum_{i=1}^{s} a_{i} W_{i}=\sum_{i=1}^{s} a_{i} Z_{i}
$$

onde para cada $i=1, \ldots, s$, temos uma solução mínima da forma

$$
(0, \ldots, 1, \ldots, 0,0, \ldots, 1, \ldots, 0)
$$

onde as entradas não nulas estão nas posições $i$ e $i+s$. Tais soluções nos dão $S$-processos nulos, portanto desnecessários. Além disso, note que se $(\alpha, \beta)$ é uma solução mínima, então $(\beta, \alpha)$ também o é, no entanto, essas soluções geram, a menos de constante, o mesmo $S$-processo mínimo. Deste ponto em diante, ao mencionarmos $S$-processos mínimos, estaremos excluindo os casos triviais e as redundâncias acima citadas.

Utilizando $o$ fato de que o semigrupo $\Gamma$ de uma curva algebróide irredutível possui condutor $\mu$, podemos refinar o algoritmo do Teorema 1.3. De 
fato, seja $\Gamma_{i}$ o semigrupo gerado pelas ordens dos elementos do conjunto $F$ em um dado passo $i$. Se $\Gamma_{i}$ possuir condutor $\mu_{i}$, então $\mu \leq \mu_{i}$. Desse modo, em cada passo, basta considerarmos os $S$-processos mínimos cujas alturas sejam inferiores a $t^{\mu_{i}-1}$

Temos assim:

Algoritmo 2.1

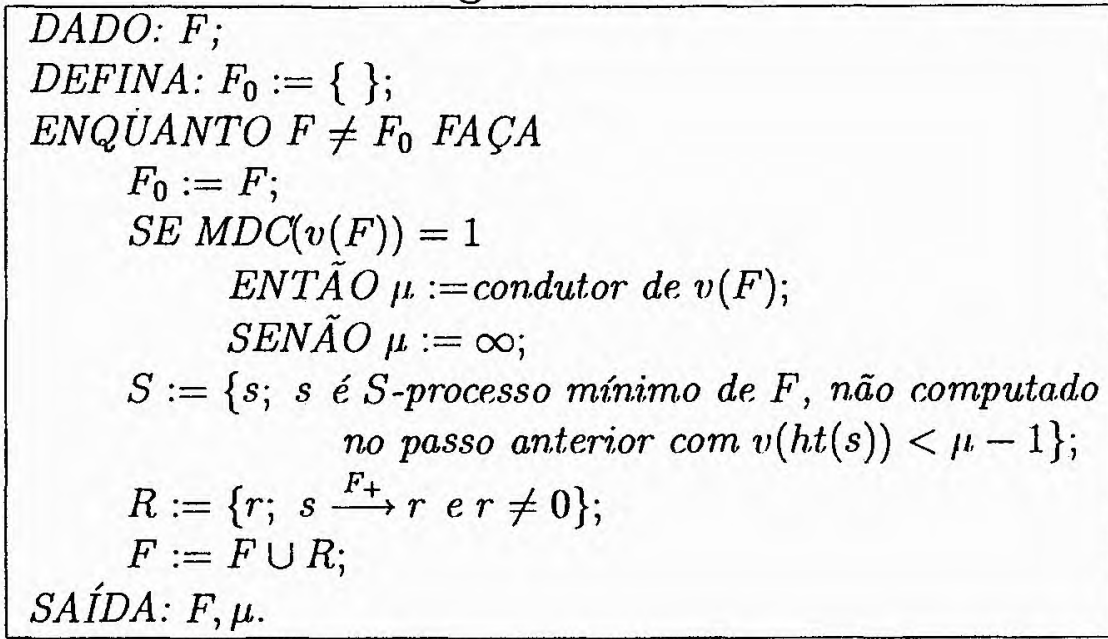

Exemplo 2.1 1. Seja

$$
C:\left\{\begin{array}{l}
x=t^{8} \\
y=t^{10}+t^{13}
\end{array}\right.
$$

Tomando $F=\{x, y\}$, o único $S$-processo mínimo a ser considerado é $y^{4}-x^{5}$.

Temos assim, que

$$
z=y^{4}-x^{5} \begin{cases}=4 t^{43}+6 t^{46}+4 t^{59}+t^{52} & \text { se } \operatorname{car}(K) \neq 2 \\ \stackrel{x^{4} y^{2}}{\longrightarrow} \stackrel{x^{6} y}{\longrightarrow} t^{61} & \text { se } \operatorname{car}(K)=2\end{cases}
$$

Independente da característica, uma análise mostra que não existe $S$ processos mínimos relevantes no próximo passo do algoritmo. Portanto, $F=\{x, y, z\}$ é uma Base Standard Mínima para $\mathcal{O}$, com

\begin{tabular}{|c|c|c|}
\hline & $\Gamma$ & $\mu$ \\
\hline $\operatorname{car}(K) \neq 2$ & $\langle 8,10,43\rangle$ & 66 \\
\hline $\operatorname{car}(\bar{K})=2$ & $\langle 8,10,61\rangle$ & 84 \\
\hline
\end{tabular}


2. Considere $\operatorname{car}(K)=0$ e seja

$$
C:\left\{\begin{array}{l}
x=t^{8}+a t^{13} \\
y=t^{10}+t^{15}
\end{array}\right.
$$

$O$ único S-processo minimo, a ser considerado no primeiro passo do algoritmo, é $y^{4}-x^{5}$, a saber

$$
z=y^{4}-x^{5}\left\{\begin{array}{c}
=(4-5 a) t^{45}+\left(6-10 a^{2}\right) t^{50}+\left(4-10 a^{3}\right) t^{55}+ \\
+\left(1-5 a^{4}\right) t^{60}-a^{5} t^{65} \text { se } a \neq \frac{4}{5}
\end{array}\right.
$$

Em. qualquer caso, não teremos $S$-processos mínimos que mereçam ser analisados nos passos seguintes. Desse modo, finalizamos o algorit.mo obtendo $F=\{x, y, z\}$ como Base Standard Minima de $\mathcal{O} e$

\begin{tabular}{|c|c|c|}
\hline & $\Gamma$ & $\mu$ \\
\hline$a \neq \frac{4}{5}$ & $\langle 8,10,45\rangle$ & 68 \\
\hline$a=\frac{4}{5}$ & $\langle 8,10,55\rangle$ & 78 \\
\hline
\end{tabular}

3. Considere $\operatorname{car}(K)=0$ e seja

$$
C:\left\{\begin{array}{l}
x=t^{8} \\
y=t^{10}+t^{13} \\
z=t^{12}+a t^{15}
\end{array}\right.
$$

Como $M D C(8,10,12)=2$ não temos condutor no primeiro passo.

$O s S$-processos mínimos e suas respectivas reduções finais módulo $F=$ $\{x, y, z\}$ são:

- $y^{4}-x^{5}=4 t^{43}+6 t^{46}+4 t^{49}+t^{52}$.

Note que a ordem deste S-processo nos indica que o condutor de $\Gamma$ é menor qûe 58, que é o condutor de $\langle 8,10,12,43\rangle \subseteq \Gamma$.

$y^{2}-x z=\left\{\begin{array}{l}(2-a) t^{23}+t^{26} \text { se } a \neq 2 \\ t^{26} \stackrel{x^{2} y}{\longrightarrow}-t^{29} \text { se } a=2 .\end{array}\right.$ 


$$
\begin{aligned}
& \text { - } z^{2}-x^{3}=\left\{\begin{array}{l}
2 a t^{27}+a^{2} t^{30} \text { se } a \neq 0 \\
0 \text { se } a=0
\end{array}\right. \\
& y^{2} z-x^{4}=\left\{\begin{array}{l}
(2+a) t^{35}+(1+2 a) t^{38}+a t^{41} \text { se } a \neq-2 \\
-3 t^{38}-2 t^{41} \stackrel{-3 x y^{3}}{\longrightarrow} 7 t^{41}+9 t^{44}+3 t^{47} \text { se } a=-2
\end{array}\right. \\
& z^{3}-x^{2} y^{2}=\left\{\begin{array}{l}
(3 a-2) t^{39}+\left(3 a^{2}-1\right) t^{42}+a^{3} t^{45} \text { se } a \neq \frac{2}{3} \\
\frac{1}{3} t^{42}+\frac{8}{27} t^{45} \stackrel{\frac{1}{3} x^{4} y}{\longrightarrow}-\frac{1}{27} t^{45} \text { se } a=\frac{2}{3}
\end{array}\right. \\
& z^{4}-y^{6}=\left\{\begin{array}{l}
4(a-1) t^{51}+6\left(a^{2}-1\right) t^{54}+4\left(a^{3}-1\right) t^{57}+ \\
+\left(a^{4}-1\right) t^{60} \text { se } a \neq 1 \\
0 \text { se } a=1 .
\end{array}\right. \\
& \text { - } z^{5}-y^{6}=(5 a-6) t^{63}+\left(10 a^{2}-15\right) t^{66}+\left(10 a^{3}-20\right) t^{69}+ \\
& +\left(5 a^{4}-15\right) t^{72}+\left(a^{5}-6\right) t^{75}-t^{78}
\end{aligned}
$$

Não efetuamos a redução do último $S$-processo, uma vez que a ordem deste supera o condutor de $\Gamma$.

Analisando as possibilidades, temos:

- Se $a=0$, então $F=\left\{t^{8}, t^{10}+t^{13}, t^{12}, 2 t^{23}+t^{26}\right\}$ é uma Base Standard Mínima para $\mathcal{O}, \Gamma=\langle 8,10,12,23\rangle$ é seu semigrupo de valores e, $\mu=38$ seu condutor.

- Se $a=2$, então $F=\left\{t^{8}, t^{10}+t^{13}, t^{12}+2 t^{15}, 4 t^{27}+4 t^{30},-t^{29}\right\}$ é Base Standard Mínima, onde $\Gamma=\langle 8,10,12,27,29\rangle$ é seu semigrupo de valores com condutor 34 .

- Se $a \neq 0$ e $a \neq 2$, então $F=\left\{t^{8}, t^{10}+t^{13}, t^{12}+a t^{15},(2-a) t^{23}+\right.$ $\left.t^{26}, 2 a t^{27}+a^{2} t^{30}\right\}$ é uma Base Standard Mínima para $K\left[\left[t^{8}, t^{10}+\right.\right.$ $\left.\left.t^{13}, t^{12}+a t^{15}\right]\right], \Gamma=\langle 8,10,12,23,27\rangle$ é o semigrupo de valores $e$ $\mu=30$ é seu condutor.

Este exemplo mostra que o semigrupo de valores de curvas algebróides irredutiveis espaciais, não depende exclusivamente dos expoentes da 
parametrização, diferente do caso de curvas planas, dadas por uma parametrização de Puiseux, veja Théorème 3.9 de [Z2].

Observação 2.2 Seja $F$ uma Base Standard Mínima para o anel local $\mathcal{O}$ de uma curva algebróide irredutível $C$. Fixemos uma mancira de efciuar a redução final, módulo $F$. Por exemplo, ordenamos os elementos de $F$ segundo suas ordens e se for possível efetuar a redução por dois $F$-produtos de potências a $F^{\alpha}$ e $b F^{\beta}$, escolhemos $a F^{\alpha}$ se a primeira coordenada, apartir da esquerda, de $\alpha-\beta$ for positiva.

Desse modo, todo elemento de $\mathcal{O}$ pode ser univocamente representado em. termos de $F$, utilizando este processo de realizar a redução final, módulo $F$..

Considere $\operatorname{car}(K)=0$ e a curva dada no item 1) do Exemplo 2.1, onde $\mathcal{O}=K[[F]]$ e $F$ é uma Base Standard Mínima $\left\{x=t^{8}, y=t^{10}+t^{13}, z=\right.$ $\left.4 t^{43}+6 t^{46}+4 t^{49}+t^{52}\right\}$.

As séries $g=4 t^{43}-14 t^{49}-17 t^{52}-6 t^{55}$ e $h=4 t^{43}-13 t^{49}-17 t^{52}-6 t^{55}$ pertencem a $\mathcal{O}$ ?

Para responder essa questão, basta computar a redução final de $g$ e $h$, módulo $F$. A saber, temos

$$
\begin{aligned}
& g \stackrel{z}{\longrightarrow}-6 t^{46}-18 t^{49}-18 t^{52}-6 t^{55} \stackrel{6 x^{2} y^{3}}{\longrightarrow} 0 \\
& h \stackrel{z}{\longrightarrow}-6 t^{46}-17 t^{49}-18 t^{52}-6 t^{55} \stackrel{6 x^{2} y^{3}}{\longrightarrow} t^{49}
\end{aligned}
$$

Portanto $g \in \mathcal{O}$, enquanto que $h \notin \mathcal{O}$.

Seja $C$ uma curva algebróide irredutível, dada por equações $f_{1}, \ldots, f_{r}$, então o semigrupo de valores $\Gamma$ de $C$ é obtido através do cálculo de $v(\bar{g}) \mathrm{com}$ $g=\bar{g}+\left\langle f_{1}, \ldots, f_{r}\right\rangle$, onde $g \in K[[\underline{X}]]$ é uma hipersuperfície.

O cálculo de $v(\bar{g})$ em termos das equações cartesianas $g, f_{1}, \ldots, f_{r}$ pode ser realizado usando a relação

$$
v(\bar{g})=\operatorname{dim}_{K} \frac{K[[\underline{X}]]}{\left\langle f_{1}, \ldots, f_{r}, g\right\rangle},
$$

e a codimensão de $\left\langle f_{1}, \ldots, f_{r}, g\right\rangle$ se resume a computar uma Base Standard para este ideal (Veja [Bec1] e [Bec2]).

Uma vez que podemos computar a ordem de um elemento de $K[[\underline{X}]$, podemos obter expressões da forma $S=F^{\alpha}-a F^{\beta}, \operatorname{com} v\left(F^{\alpha}\right)=v\left(F^{\beta}\right)$ onde $\alpha, \beta \in \mathbb{N}^{\sharp F}$ e $F \subseteq \mathcal{M}_{\underline{X}}^{*}$. Sabemos que' existe um único $a \in K^{*}$ de modo 
que $S$ é um $S$-processo, i.e., $v(S)>v\left(F^{\alpha}\right)=v\left(F^{\beta}\right)$, tal constante pode ser determinada, computando uma Base Standard para o ideal $\left\langle f_{1}, \ldots, f_{r}, F^{\alpha}-\right.$ $\left.a F^{\beta}\right\rangle \subset K[[\underline{X}]]$ (Veja [Bec1] e [Bec2]) e analisando para que valor de $a \in K^{*}$ temos

$\left.\operatorname{dim}_{K} \frac{K[[\underline{X}]]}{\left\langle f_{1}, \ldots, f_{r}, F^{\alpha}-a F^{\beta}\right\rangle}\right\rangle \operatorname{dim}_{K} \frac{K[[\underline{X}]]}{\left\langle f_{1}, \ldots, f_{r}, F^{\alpha}\right\rangle}=\operatorname{dim}_{K} \frac{K[[\underline{X}]]}{\left\langle f_{1}, \ldots, f_{r}, F^{\beta}\right\rangle}$.

Do mesmo modo, podemos efetuar a redução de um eleménto de $K[[\underline{X}]]$ módulo $F \subseteq \mathcal{M}_{X}^{*}$, quando $C$ é dada por. equações cartesianas. Portanto, podemos aplicar o algoritmo para obter uma Base Standard de álgebras para $\mathcal{O}$.

Note no entanto, que teremos controle apenas sobre a ordem dos elementos, mas não de seus termos. Desse modo, no caso em que $C$.é dada por equações cartesianas, podemos obter uma Base Standard Mínima para $\mathcal{O}$, mas não a Base Standard Reduzida.

Exemplo 2.2 Considere car $(K)=0$ e seja $C$ a curva algebróide irredutivel dada pela série irredutivel $f(X, Y)=Y^{8}-4 X^{3} Y^{6}-8 X^{5} Y^{5}+\left(6 X^{6}-\right.$ $\left.26 X^{7}\right) Y^{4}+\left(16 X^{8}-24 X^{9}\right) Y^{3}+\left(-4 X^{9}+36 X^{10}-20 X^{11}\right) Y^{2}+\left(-8 X^{11}+\right.$ $\left.16 X^{12}-8 X^{13}\right) Y+X^{12}+6 X^{13}+21 X^{14}-X^{15}$.

Como $C$ é uma curva plana, temos

$$
v(\bar{g})=\operatorname{dim}_{K} \frac{K[[X, Y]]}{\langle f, g\rangle}=I(f, g)=\operatorname{ord}_{X}\left(R_{Y}(f, g)\right),
$$

onde, $I(f, g)$ denota a multiplicidade de interseçâo de $g$ com $f_{x:} R_{Y}(f, g)$ o resultante em. $Y$ de $f$ e. $g$ e $\vec{g}=g+\langle f\rangle$, com $g \in K[[X, Y]]$.

Apliquemos o algoritmo para $F=\{X, Y\}$, a fim de obter uma Base. Standard para $\mathcal{O}$.

Como $I(f, X)=8$ e $I(f, Y)=12$, não temos condutor no primeiro passo.

$O$ único $S$-processo mínimo não trivial nesse passo é da forma $Y^{2}-a X^{3}$. Como $R_{Y}\left(f, Y^{2}-a X^{3}\right)=(a-1)^{8} X^{24}+(a-1)^{4}\left(-52 a^{2}+8 a+12\right) X^{25}+$ $\left(-40 a^{5}+494 a^{4}-1256 a^{3}+500 a^{2}+480 a+78\right) X^{26}+\cdots$, a unica escolha para obtermos um $S$-processo é $a=1$, e desta forma $I\left(f, Y^{2}-X^{3}\right)=26$, passamos para o segundo passo com $F=\left\langle X, Y, Z=Y^{2}-X^{3}\right\rangle$.

Como o semigrupo $\langle 8,12,26\rangle$ não possui condutor, devemos analisar todos os $S$-processos mínimos de $F$, cujo resultante com $f$ são:

$R_{Y}\left(f, Z^{2}-a_{1} X^{2} Y^{3}\right)=\left(a_{1}-4\right)^{8} X^{52}+\left(18 a_{1}^{8}+632 a_{1}^{7}-13156 a_{1}^{6}+17248 a_{1}^{5}+\right.$ $\left.457280 a_{1}^{4}-10782772 a_{1}^{3}-2153472 a_{1}^{2}-1417216 a_{1}-16384\right) X^{53}+\cdots$ 
$R_{Y}\left(f, Z^{2}-a_{2} X^{5} Y\right)=\left(a_{2}-4\right)^{8} X^{52}+\left(6 a_{2}^{8}-128 a_{2}^{7}+769 a_{2}^{6}-2336 a_{2}^{5}+\right.$ $\left.11840 a_{2}^{4}-130048 a_{2}^{3}-384000 a_{2}^{2}-1122304 a_{2}-80384\right) X^{53}+\cdots$

$R_{Y}\left(f, X Z^{2}-a_{3} Y^{5}\right)=\left(a_{3}-4\right)^{8} X^{60}+\left(30 a_{3}^{8}+4272 a_{3}^{7}-44260 a_{3}^{6}-149536 a_{3}^{5}+\right.$ $\left.2287168 a_{3}^{4}-3451904 a_{3}^{3}-7003136 a_{3}^{2}-1712128 a_{3}-16384\right) X^{61}+\cdots$

$R_{Y}\left(f, Y Z^{2}-a_{4} X^{8}\right)=\left(a_{4}-4\right)^{8} X^{64}+\left(-120 a_{4}^{7}-9956 a_{4}^{6}+42592 a_{4}^{5}+\right.$ $\left.9792 a_{4}^{4}-590848 a_{4}^{3}+1614848 a_{4}^{2}-2138112 a_{4}+376832\right) X^{65}+\cdots$

$$
\begin{aligned}
& R_{Y}\left(f, Z^{4}-a_{5} X Y^{8}\right)=\left(a_{5}-16\right)^{8} X^{104}+\cdots \\
& R_{Y}\left(f, Z^{4}-a_{6} X^{13}\right)=\left(a_{6}-16\right)^{8} X^{104}+\cdots \\
& R_{Y}\left(f, Z^{6}-a_{7} Y^{13}\right)=\left(a_{7}-64\right)^{8} X^{156}+\cdots
\end{aligned}
$$

Note que para obtermos $S$-processos, devemos tormar $a_{1}=a_{2}=a_{3}=$ $a_{4}=4, a_{5}=a_{6}=16$ e $a_{7}=64$, mas deste modo $I\left(f, Z^{2}-4 X^{5} Y\right)=53$ e como o condutor do semigrupo $\langle 8,12,26,53\rangle$ é 84 , podemos dispensar os três últimos $S$-processos.

Uma verificação nos indica que no próximo passo não teremos $S$-processos mínimos, não triviais, com altura menor que 84.

Temos assim, que $F=\left\{X, Y, Z=Y^{2}-X^{3}, W=Z^{2}-4 X^{5} Y, U_{1}=\right.$ $\left.Z^{2}-4 X^{2} Y^{3}, U_{2}=X Z^{2}-4 Y^{5}, U_{3}=Y Z^{2}-4 X^{8}\right\}$ é uma Base Standard para $\mathcal{O}$. Como $I\left(f, U_{1}\right)=I(f, W), I\left(f, U_{2}\right)=I(f, X W)$ e $I\left(f, U_{3}\right)=$ $I(f, Y W)$, temos que $\{X, Y, Z, W\}$ é uma Base Standard Mínima para $\mathcal{O}$ e $\Gamma=\langle 8,12,26,53\rangle$ é o semigrupo de valores associado à. $C$.

$\mathrm{O}$ algoritmo apresentado anteriormente, não se restringe a um tipo particular de curva algebróide irredutível. De fato, podemos aplicá-lo a qualquer curva algebróide irredutível sobre qualquer corpo algebricamente fechado $K$, independentemente de sua característica.

Uma Base Standard do anel local $\mathcal{O}$ de uma curva algebróide irredutível, depende da equação que a define e da parametrização obtida, no entanto, o semigrupo de valores não, uma vez que este é obtido pelas ordens de elementos de.uma Base Standard Mínima de $\mathcal{O}$ e não depende das expressões destes.

Definição 2.2 Dizemos que duas curvas algebróides irredutíveis em $K[[\underline{X}]]$ são equisingulares, se elas possuem o mesmo semigrupo de valores.

Obter 0 .semigrupo de valores $\Gamma$ de uma curva algebróide é de grande importância, pois através dele, podemos obter várias informações sobre a 
natureza da curva em questão. Temos assim, um algoritmo para detcrminar se duas curvas algebróides irredutíveis são equisingulares ou não.

Além disso, podemos verificar se uma curva algebróide irredutível é Gorenstein, uma vez que estas são definidas através de propriedades do scmigrupo, a saber, uma curva é Gorenstein se, e somente sc, $\sharp(\mathbb{N} \backslash \Gamma)=\frac{\mu}{2}$.

Dentre as curvas de Gorenstein, uma categoria importante, são as curvas de interseção completa, ou seja, aquelas que são dadas por $f_{1}, \ldots, f_{n-1}$, com $f_{i} \in \mathcal{M}_{\underline{X}}^{*} \subset K\left[\left[X_{1}, \ldots, X_{n}\right]\right]$. Mesmo sem entrar em detallies, mencionamos que se o semigrupo de valores $\left\langle v_{0}, v_{1}, \ldots, v_{g}\right\rangle$ de uma curva é um semigrupo de interseção completa, então a curva certamente é de interseção completa.

Delorme, em [D1], apresenta um algoritmo para decidir se um semigrupo de $\mathbb{N}$ é um semigrupo de interseção completa.

Exemplo 2.3 Considere $\operatorname{car}(K)=0$ e seja

$$
C:\left\{\begin{array}{l}
x=t^{6} \\
y=t^{8}+2 t^{9} \\
z=t^{10}+t^{11}
\end{array}\right.
$$

Aplicando o algoritmo para obter uma Base Standard Mínima para $\mathcal{O}$, obtem.os $F=\{x, y, z, w, y\}$, onde $w=x z-y^{2}=-3 t^{17}-4 t^{18}$ e $u=y z-x^{3}=$ $3 t^{19}+2 t^{20}$. Desse modo, o semigrupo associado à $C$ é $\Gamma=\langle 6,8,10,17,19\rangle$, cujo condutor é $\mu=22$. Uma vcz que $\mathbb{N} \backslash \Gamma=\{1,2,3,4,5,7,9,11,13,15,21\}$, a curva é Gorenstein.

$O$ algoritmo de Delorme, citado anteriormente, indica que $\Gamma$ não é um. semigrupo de interseção completa. No entanto, esse é um exemplo de que podemos ter curvas de interseção completa sem que o semigrupo associàdo o seja, como mostram Herzog e Kunz, em. [HK].

\subsubsection{Semigrupos de Curvas Planas}

Vimos que podemos encontrar uma Base Standard Mínima para qualquer curva algebróide irredutível $C$. Entretanto, no caso de curvas planas e quando $\operatorname{car}(K)$ não divide a multiplicidade de $C$, Azevedo e Zariski, Abhyankar e Moh, também descrevem métodos para encontrar uma Base Standard Mínima, tais métodos serão apresentados e comparados com nosso algoritmo mais adiante.

Seja $C$ uma curva algebróide irredutível plana com multiplicidade $v_{0}$. Pelo Teorema de Preparação de Weierstrass (Veja Prop. 3.3 de [Ru]), $C$ pode ser 
dada por uma série irredutível

$$
f(X, Y)=Y^{v_{0}}+\sum_{i=1}^{v_{0}} a_{i}(X) Y^{v_{0}-i}
$$

e pelo Teorema de Newton-Puiseux (Veja Prop. 4.4 de [Ru]), por uma parametrização de Puiseux

$$
C:\left\{\begin{array}{l}
x=t^{v_{0}} \\
y=t^{v_{1}}+\sum_{i>v_{1}} b_{i} t^{i},
\end{array}\right.
$$

com $b_{i} \in K$. Podemos supor que $v_{0}<v_{1}$ e que $v_{0} \backslash v_{1}$.

Vamos nos referir a uma curva algebróide irredutível plana simplesmente por ramo.

Definimos as seguintes sequiências de inteiros:

$$
\begin{array}{lll}
\beta_{0}=v_{0}, & e_{0}=v_{0}, & n_{0}=1, \\
\beta_{1}=v_{1}, & e_{1}=\operatorname{MDC}\left(e_{0}, \beta_{1}\right), & n_{1}=\frac{e_{0}}{e_{1}} \\
\beta_{j}=\min \left\{i ; b_{i} \neq 0 \text { e } e_{j-1} \backslash \chi i\right\}, & e_{j}=\operatorname{MDC}\left(e_{j-1}, \beta_{j}\right), & n_{j}=\frac{e_{j-1}}{e_{j}} .
\end{array}
$$

Cómo observamos anteriormente, pela primitividade da parametrização, existe $g \geq 1$, tal que $e_{g}=1$.

A seqüência $\left(\beta_{0}, \beta_{1}, \ldots, \beta_{g}\right)$ é chamada seqüêncịa característica de $C$.

\section{MÉTODO DE AZEVEDO-ZARISKI}

Sejam $\omega$ uma raiz $v_{0}$-ésima primitiva da unidade e $\varphi_{\alpha} \circ K$-automorfismo de $K[[t]]$ definido por $\varphi_{\alpha}(t)=\omega^{\alpha} t$.

Defina

$$
Z_{k}=\sum_{\beta_{1} \leq i<\beta_{k}} b_{i} X^{\frac{i}{v_{0}}}
$$

$\operatorname{com} k=2, \ldots, g \mathrm{e}$

$$
P_{k}(Y)=\prod_{\alpha=1}^{n_{0} n_{1} \ldots n_{k-1}}\left(Y-\varphi_{\alpha}\left(Z_{k}\right)\right)
$$

Azevedo, em $[\mathrm{Az}]$, e Zariski, em $[\mathrm{Z} 2]$, mostram que $\left\{X, Y, P_{k}(Y) ; k=\right.$ $2, \ldots, g\}$ são séries cujas multiplicidades de interseção com $f(X, Y)$, nos dão o sistema mínimo de geradores do semigrupo $\Gamma$ da curva. 


\section{MÉTODO DE ABHYANKAR-MOH}

Seja $d \in \mathbb{N}$, tal que $d \mid v_{0}$. Definimos a d-ésima aproximação de raízes de $f$, como sendo a série

$$
\sqrt[d]{f}=Y^{\frac{v_{0}}{d}}+\sum_{i=1}^{\frac{v_{0}}{d}} c_{i}(X) Y^{\frac{v_{0}}{d}-i},
$$

onde $c_{i}(X) \in K[[X]]$ são obtidos univocamnute pelas relaçõns

$$
a_{i}(X)=d c_{i}(X)+\sum_{\sum_{j=1}^{i-1} j \delta_{j}=i} \alpha_{\delta_{1}, \ldots, \delta_{i-1}} c_{1}(X)^{\delta_{1}} \cdots, c_{i-1}(X)^{\delta_{i-1}}
$$

$i=1, \ldots, \frac{v_{0}}{d}$, onde

$$
\alpha_{\delta_{1}, \ldots, \delta_{i-1}}=\left(\begin{array}{c}
d \\
\delta_{1}+\cdots+\delta_{i-1}
\end{array}\right) \frac{\left(\sum_{j=1}^{i-1} \delta_{j}\right) !}{\prod_{j=1}^{i-1}\left(\delta_{j} !\right)} .
$$

Abhyankar e Moh em [AM], provam a existência e a unicidade das aproximações de raízes de $f$, e que as multiplicidades de interseção de $X$ e

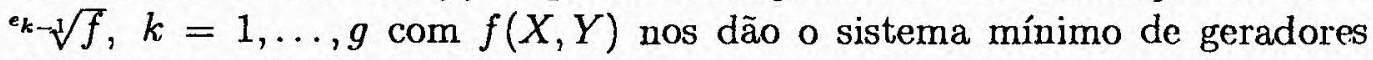
do semigrupo $\Gamma$ da curva.

\section{COMPARAÇÃO DOS MÉTODOS}

Embora o algoritmo que apresentamos, o resultado de Azevedo-Zariski e o de Ablyyankar-Moh nos dêem uma Base Staudard Mínima para $\mathcal{O}$, estas bases ıão são necessariamente as mesmas.

Considere o seguinte exemplo

$$
C:\left\{\begin{array}{l}
x=t^{8} \\
y=t^{12}+t^{14}+t^{15}
\end{array}\right.
$$

cuja equação cartesiana é $f(X, Y)=Y^{8}-4 X^{3} Y^{6}-8 X^{5} Y^{5}+\left(6 X^{6}-26 X^{7}\right) Y^{4}+$ $\left(16 X^{8}-24 X^{9}\right) Y^{3}+\left(-4 X^{9}+36 X^{10}-20 X^{11}\right) Y^{2}+\left(-8 X^{11}+16 X^{12}-8 X^{13}\right) Y+$ $X^{12}+6 X^{13}+21 X^{14}-X^{15}$.

Couforme o Exemplo 2.2, vemos que nosso algortimo forneceu a Base Standard Mínima $F=\left\{x, y, z=y^{2}-x^{3}, w=z^{2}-4 x^{5} y=\left(y^{3}-x^{3}\right)^{2}-4 x^{5} y\right\}$, enquanto que o método de Abhyankar-Moh, nos fornece $F=\{x, y, z=$ $\left.y^{2}-x^{3}, w_{1}=z^{2}-4 x^{5} y-13 x^{7}\right\}$ e o método de Azevedo-Zariski, $F=\{x, y, z=$ $\left.y^{2}-x^{3}, w_{2}=z^{2}-4 x^{5} y-x^{7}\right\}$. 


\section{OTIMIZAÇÃO DO ALGORITMOO}

Enquanto que os métodos de Azevedo-Zariski e de Abhyankar-Molı, dêem expressões explícitas, a priori, dos elementos de uma Base Standard Mínima de $\mathcal{O}$, o algoritmo que apresentamos, calcula, eventualmente, vários $S$-processos mínimos que são redundantes ou desnecessários.

Quando $\operatorname{car}(K) \backslash v_{0}$, mostraremos abaixo, como nosso algoritmo pode ser otimizado, evitando cálculos de $S$-processos dispensáveis.

Inicialmente, seja $h, \in K[[t]]$ uma série da forma

$$
h=a_{1} t^{b_{1} c_{1}}+\left(c_{1}\right)+a_{2} t^{b_{2} c_{2}}+\left(c_{2}\right)+\cdots+a_{k} t^{b_{k} c_{k}}+\left(c_{k}\right),
$$

com $a_{i} \in K^{*}, 0<c_{i+1}<c_{i}$ para todo $i \geq 1, c_{j} \mid c_{i}$ para $i \leq j$ e onde a expressão $a_{i} t^{b_{i} c_{i}}+\left(c_{i}\right)$ representa uma soma de termos cujos expoentes são divisíveis por $c_{i}$, mas não por $c_{i-1}$.

Note que o primeiro termo em $h^{p}$ onde $p$ é um inteiro positivo, cujo expoente é divisível por $c_{k}(k>1)$ é dado por

$$
\text { (*) } \quad p a_{1}^{p-1} a_{k} t^{(p-1) b_{1} c_{1}+b_{k} c_{k}} \text {. }
$$

Recordemos alguns fatos acerca do semigrupo $\Gamma=\left\langle v_{0}, v_{1}, \ldots, v_{g}\right\rangle$, de $1 \mathrm{um}$ ramo $C$. Tais fatos podem ser encontrados, por exemplo, em [Z2] on em [An].

A seqüência $e_{0}>e_{1}>\cdots>e_{g}=1$, definida no ínicio desta subseção, em termós da seqüência característica $\beta_{0}<\beta_{1}<\cdots<\beta_{g}$, pode também, ser obtida do seguinte modo:

$$
\begin{gathered}
e_{0}=v_{0}, \\
e_{i}=\operatorname{MDC}\left(e_{i-1}, v_{i}\right) .
\end{gathered}
$$

Uma vez que $\operatorname{car}(K) \not\left\langle v_{0}\right.$, podemos considerar que $C$ seja dada em termos de uma parametrização de Puiseux da forma

$$
C:\left\{\begin{array}{l}
x=t^{\beta_{0}} \\
y=t^{\beta_{1}}+\left(e_{1}\right)+a_{2} t^{\beta_{2}}+\left(e_{2}\right)+\cdots+a_{g} t^{\beta_{g}}+\left(e_{g}\right)
\end{array}\right.
$$

com $a_{i} \neq 0, \beta_{0}=v_{0}=n_{0} n_{1} \cdots n_{g}$ e $\beta_{1}=v_{1}$. Além disto, temos que

$$
v_{i+1}>n_{i} v_{i} \quad e \quad n_{i+1} v_{i+1} \in\left\langle v_{0}, \ldots, v_{i}\right\rangle
$$

para todo $i=0, \ldots, g-1$. 
Os semigrupos $\Gamma_{i}=\left\langle\frac{v_{0}}{e_{i}}, \ldots, \frac{v_{i}}{e_{i}}\right\rangle$, onde $i=0, \ldots, g$, possuem condutor

$$
\mu_{i}=\sum_{j=1}^{i}\left(n_{j}-1\right) \frac{v_{j}}{e_{i}}-\frac{v_{0}}{e_{i}}+1
$$

Como

$$
\begin{aligned}
n_{i} v_{i} & =\left(n_{i}-1\right) v_{i}+v_{i}>\sum_{j=1}^{i}\left(n_{j}-1\right) v_{j}+v_{1} \geq \\
& \geq e_{i}\left(\sum_{j=1}^{i}\left(n_{i j}-1\right) \frac{v_{j}}{e_{i}}+\frac{v_{0}}{e_{i}}+1\right)>e_{i} \mu_{i}, .
\end{aligned}
$$

temos que todo inteiro múltiplo de $e_{i}$, maior que $n_{i} v_{i}$, pertence à $e_{i} \Gamma_{i}$.

Além disso, a sequiência característica $\beta_{0}<\beta_{1}<\cdots<\beta_{g}$, se relaciona com os elementos do sistema mínimo de geradores do semigrupo $\Gamma=\left\langle v_{0}, v_{1}, \ldots, v_{g}\right\rangle$ de $C$ através da equação

$$
v_{i+1}=\sum_{j=1}^{i}\left(n_{j}-1\right) v_{j}+\beta_{i+1}=n_{i} v_{i}-\beta_{i}+\beta_{i+1}
$$

Proposição 2.1 Seja C um ramo dado por uma parametrização de Puiseux

$$
C:\left\{\begin{array}{l}
x=a_{0} t^{\beta_{0}} \\
y=a_{1} t^{\beta_{1}}+\left(e_{1}\right)+a_{2} t^{\beta_{2}}+\left(e_{2}\right)+\cdots+a_{g} t^{\beta_{g}}+\left(e_{g}\right) .
\end{array}\right.
$$

Uma Base Standard Mínima para $\mathcal{O}$ é $\left\{h_{0}, h_{1}, \ldots, h_{g}\right\}$, com $h_{0}=x, h_{1}=$ $y$ e $h_{i+1}$ obtido por uma redução final, módulo $\left\{h_{0}, h_{1}, \ldots, h_{i}\right\}$, do $S$-processo

$$
\frac{1}{n_{i} a_{i}^{n_{i}-1}} h_{i}^{n_{i}}-\frac{a_{i}}{\left.n_{i}\right\rfloor \prod_{j=0}^{i-1} a_{j}^{\alpha_{j}}} \prod_{j=0}^{i-1} h_{j}^{\alpha_{j}}
$$

para todo $i=1, \ldots, g-1$ e $\sum_{j=0}^{i-1} \alpha_{j} v_{j}=n_{i} v_{i}$.

Além. disso,

$$
h_{i+1}=\sum_{j=i+1}^{g} a_{j} t^{\gamma_{i}+\beta_{j}}+\left(e_{j}\right)
$$

$\operatorname{com} \gamma_{i}=\sum_{l=1}^{i}\left(n_{l}-1\right) v_{l}$

Dem.: Procedemos por indução sobre $i$.

Para $i=1$, tome $\alpha_{0} \in \mathbb{N}$ tal que $\alpha_{0} v_{0}=n_{1} v_{1}$. Assim, $x^{\alpha_{0}}=a_{0}^{\alpha_{0}} t^{n_{1} v_{1}} \mathrm{e}$

$$
\begin{gathered}
y^{n_{1}}=a_{1}^{n_{1}} t^{n_{1} v_{1}}+\left(e_{1}\right)+n_{1} a_{1}^{n_{1}-1} a_{2} t^{\left(n_{1}-1\right) v_{1}+\beta_{2}}+\left(e_{2}\right)+\cdots \\
\cdots+n_{1} a_{1}^{n_{1}-1} a_{g} t^{\left(n_{1}-1\right) v_{1}+\beta_{g}}+\left(e_{g}\right) .
\end{gathered}
$$


Temos, deste modo, o $S$-processo

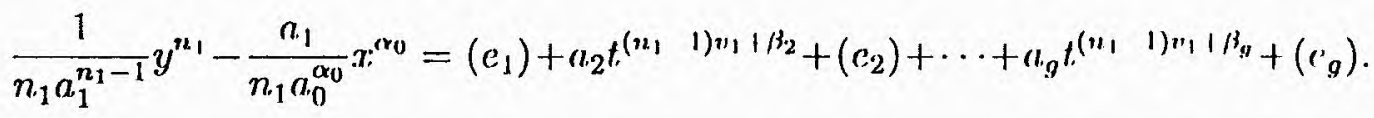

Uma vez que, na expressão acima, os expoentes divisíveis por $e_{1}$, são maiores do que $n_{1} v_{1}$, sempre podemos obter um elemento da forma $x^{\alpha} y^{\beta}$ que possui tal ordem.

Note que em $x^{\alpha} y^{\beta}$, onde $\alpha v_{0}+\beta v_{1}>n_{1} v_{1}$, o menor expoente de $t$ não divisível por $e_{k}(k>1)$ é por $(*), \alpha v_{0}+(\beta-1) v_{1}+\beta_{k}$, que em vista da desigualdade anterior nos dá

$$
\alpha v_{0}+(\beta-1) v_{1}+\beta_{k}>\left(n_{1}-1\right) v_{1}+\beta_{k}
$$

Ou seja, ao efetuarmos a redução do $S$-processo por produtos $x^{\alpha} y^{\beta}$ como acima, podemos sempre eliminar os termos com expoente divisível por $e_{1}$, sem alterar os termos da forma $a_{k} t^{\left(n_{1}-1\right) v_{1}+\beta_{k}}$, conseqüentemente, a redução final do $S$-processo em questão, módulo $\left\{h_{0}, h_{1}\right\}$ é da forma

$$
h_{2}=a_{2} t^{\left(n_{1}-1\right) v_{1}+\beta_{2}}+\left(e_{2}\right)+\cdots+a_{g} t^{\left(n_{1}-1\right) v_{1}+\beta_{g}}+\left(e_{g}\right),
$$

cuja ordem é $\left(n_{1}-1\right) v_{1}+\beta_{2}=v_{2}$, provando assim a proposição para $i=1$.

Vamos supor o resultado válido para todo $1 \leq j \leq i<g-1$, isto é,

$$
h_{j}=\sum_{k=j}^{g} a_{k} t^{\sum_{l=1}^{j-1}\left(n_{l}-1\right) v_{l}+\beta_{k}}+\left(e_{k}\right),
$$

cuja ordem é $\sum_{l=1}^{j-1}\left(n_{l}-1\right) v_{l}+\beta_{j}=v_{j}$.

$O$ termo líder de $\frac{1}{n_{i} a_{i}^{n_{i}-1}} h_{i}^{n_{i}}$ é

$$
\frac{1}{n_{i} a_{i}^{n_{i}-1}} a_{i}^{n_{i}} t^{n_{i} v_{i}}=\frac{a_{i}}{n_{i}} t^{n_{i} v_{i}}
$$

e por $(*)$, o seı termo de menor ordem, não divisível por $c_{k}(k>i)$ é

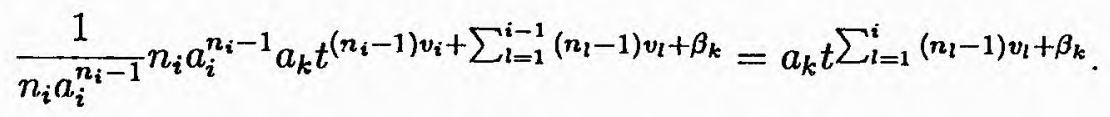

Sejam $\alpha_{j}, \operatorname{com} j=0, \ldots, i-1$, tais que $n_{i} v_{i}=\sum_{j=0}^{i-1} \alpha_{j} v_{j}$. 
Então o termo líder de $\frac{a_{i}}{n_{i} \prod_{j=0}^{i-1} a_{j}^{\alpha_{j}}} \prod_{j=0}^{i-1} h_{j}^{\alpha_{j}}$ é o produto dos termos líderes de cada $h_{j}^{\alpha_{j}}$, o1 seja,

$$
\frac{a_{i}}{n_{i} \prod_{j=0}^{i-1} a_{j}^{\alpha_{j}}} \prod_{j=0}^{i-1} a_{j}^{\alpha_{j}} t^{\alpha_{j} v_{j}}=\frac{a_{i}}{n_{i}} t^{n_{i} v_{i}}
$$

O termo em $t$, de menor ordem no produt,o $\prod_{j=0}^{i-1} h_{j}^{\alpha_{j}}$, cujo expoente 11 ão é divisível por $e_{k}$, é obtido tomando o termo de menor ordem não divisível por $e_{k}$ em um fator e os termos líderes dos demais. Assim, por (*) sua ordem será

$$
\begin{aligned}
\sum_{j=0, j \neq l}^{i-1} \alpha_{j} v_{j}+ & \left(\alpha_{l}-1\right) v_{l}+\sum_{j=1}^{l-1}\left(n_{j}-1\right) v_{j}+\beta_{k}= \\
& =\sum_{j=0}^{i-1} \alpha_{j} v_{j}-v_{l}+\sum_{j=1}^{l-1}\left(n_{j}-1\right) v_{j}+\beta_{k}= \\
& =n_{i} v_{i}-\beta_{l}+\beta_{k}>n_{i} v_{i}-\beta_{i}+\beta_{k}=\sum_{j=1}^{i}\left(n_{j}-1\right) v_{j}+\beta_{k} .
\end{aligned}
$$

Desse modo,

$$
\frac{1}{n_{i} a_{i}^{n_{i}-1}} h_{i}^{n_{i}}-\frac{a_{i}}{n_{i} \prod_{j=0}^{i-1} a_{j}^{\alpha_{j}}} \prod_{j=0}^{i-1} h_{j}^{\alpha_{j}}
$$

é de fato um $S$-processo e o termo de menor expoente não divisível por $e_{k}(k>i)$ é o respectivo termo proveuiente de $\frac{1}{n_{i} a_{i}^{n_{i}-1}} h_{i}^{n_{i}}$, ou seja, o $S$-processo acima é da forma

$$
\left(e_{i}\right)+\sum_{j=i+1}^{g} a_{j} t \sum_{l=1}^{i}\left(n_{l}-1\right) v_{l}+\beta_{j}+\left(e_{j}\right) .
$$

Uma vez que na série acima, os expoentes divisíveis por $e_{i}$ são superiores a $n_{i} v_{i}$, podemos obter elementos da forma $\prod_{j=0}^{i} h_{j}^{\gamma_{j}}$ que possuem tais ordens. Novamente por $(*)$, o menor expoente divisível por $e_{k}(k>i)$ nesses produtos, é da forma

$$
\begin{gathered}
\sum_{j=0, j \neq l}^{i} \gamma_{j} v_{j}+\left(\gamma_{l}-1\right) v_{l}+\sum_{j=1}^{l-1}\left(n_{j}-1\right) v_{j}+\beta_{k}>n_{i} v_{i}-\beta_{l}+\beta_{k} \geq \\
\geq n_{i} v_{i}-\beta_{i}+\beta_{k}=\sum_{j=1}^{i}\left(n_{j}-1\right) v_{j}+\beta_{k} .
\end{gathered}
$$

Assim, as reduções não alteram o termo de menor ordem, no $S$-processo, que é divisível por $e_{k}(k>i)$.

Portanto, a reḍução final é da forma

$$
h_{i+1}=\sum_{j=i+1}^{g} a_{j} t^{\sum_{l=1}^{i}\left(n_{l}-1\right) v_{l}+\beta_{j}}+\left(e_{j}\right),
$$


cuja ordem é $\sum_{l=1}^{i}\left(n_{l}-1\right) v_{l}+\beta_{i+1}=v_{i+1}$.

\subsection{O Módulo de Diferenciais de uma Curva}

No que segue, $K$ é um corpo algebricamente fechado com $\operatorname{car}(K)=0$.

Seja $C$ uma curva algebróide irredutível sobre $K$, dada por $f_{1}, \ldots, f_{r}$ com $f_{i} \in \mathcal{M}_{\underline{X}}^{*} \subset K\left[\left[X_{1}, \ldots, X_{n}\right]\right], \mathcal{O}$ o anel local de $C$ e $\overline{\mathcal{O}}$ sen fecho integral. Como vimos na seção anterior, podemos considerar $\mathcal{O}$ como uma subálgebra de $K[[t]]$, por meio do $K$-homomorfismo de álgebras $\varphi$ definido em (2.1).

Considere a $K$-derivação usual $d$, definida em um monômio $x_{1}^{\alpha_{1}} \cdots x_{n}^{\alpha_{n}}$ por

$$
d\left(x_{1}^{\alpha_{1}} \cdots \cdot x_{n}^{\alpha_{n}}\right)=\sum_{i=1}^{n} \alpha_{i} x_{1}^{\alpha_{1}} \cdots \cdot x_{i}^{\alpha_{i}-1} \cdot \cdots x_{n}^{\alpha_{n}} d x_{i}
$$

e estendendo por linearidade.

Definimos o $\mathcal{O}$-módulo de diferenciais da curva $C$, como sendo

$$
\mathcal{O} d \mathcal{O}=\mathcal{O} d x_{1}+\cdots+\mathcal{O} d x_{n}
$$

Observação $2.3 \quad$ 1. Note que em $\mathcal{O}$, temos

$$
f_{1}=\cdots=f_{r}=0
$$

assim,

$$
\sum_{i=1}^{n} f_{1_{x_{i}}} d x_{i}=\cdots=\sum_{i=1}^{n} f_{n_{x_{i}}} d x_{i}=0 .
$$

2. $O \mathcal{O}$-módulo $\mathcal{O} d \mathcal{O}$ não é necessariamente livre de torção.

De fato, seja $C$ a curva dada por $f(X, Y)=Y^{v_{0}}-X^{v_{1}}$ com $\min \left\{v_{0}, v_{1}\right\}>1$ e $\Omega=v_{0} x d y-v_{1} y d x \neq 0$.

Temos assim, que

$$
\begin{aligned}
y^{v_{0}-1} \Omega & =v_{0} x y^{v_{0}-1} d y-v_{1} y^{v_{0}} d x= \\
& =v_{0} x y^{v_{0}-1} d y-v_{1} x^{v_{1}} d x= \\
& =x\left(v_{0} y^{v_{0}-1} d y-v_{1}^{v_{1}-1} d x\right)= \\
& =x\left(f_{Y} d y-f_{X} d x\right)=0
\end{aligned}
$$


Denotaremos o $\mathcal{O}$-submódulo de torção de $\mathcal{O} d \mathcal{O}$ por $T$. Veremos adiante. que tal submódulo reserva propriedades importantes a respeito da curva $C$.

Denotemos $x_{i}^{\prime}=p_{i}^{\prime}(t)=\frac{d p_{i}(t)}{d t}$ e consideremos a aplicação

$$
\psi: \underset{\sum_{i=1}^{n} g_{i} d x_{i}}{\mathcal{O} d \mathcal{O}} \stackrel{\mapsto}{\sum_{i=1}^{n} \varphi(g) x_{i}^{\prime}}
$$

onde $g \in \mathcal{O}$ e $\varphi$ é o monomorfismo de $K$-álgebras definido em (2.1).

Se identificarmos $\mathcal{O} \operatorname{com} \varphi(\mathcal{O})$, então a aplicação acima é um homomorfismo de $\mathcal{O}$-módulos. Além disso, $\operatorname{Ker}(\psi)=T$, ou seja,

$$
\frac{\mathcal{O} d \mathcal{O}}{T} \cong \operatorname{Im}(\psi)
$$

Uma vez que nossa teoria de Base Standard para Módulos foi desenvolvida para módulos livres de torção em $K[[\underline{X}]]$, ntilizaremos para efeito computacional o $\varphi(\mathcal{O})$-módulo $\operatorname{Im}(\psi)$ em $K[[t]]$.

Seja $\Omega \in \mathcal{O} d \mathcal{O} \backslash T$, definimos a ordem de $\Omega$ como $v(\Omega)=v(\psi(\Omega)$ ), onde $v$ é a valorização discreta de $\overline{\mathcal{O}}(=K[[t]])$.

A proposição abaixo, garante que o $\mathcal{O}$-módulo $\mathcal{O} d \mathcal{O}$, mais exatament. o $\varphi(\mathcal{O})$-módulo $\operatorname{Im}(\psi)$, possui Base Standard de $\mathcal{O}$-módulos.

Proposição 2.2 Seja $A \subseteq K[[X]]$ uma subálgebra. Todo A-submódulo $M$ de $K[[X]]$ é finitamente. gerado.

Dem.: Se $\Gamma=\left\{\operatorname{ord}_{X}(a) ; a \in A\right\}$ e $\Xi=\left\{\operatorname{ord}_{X}(m) ; m \in M\right\}$, então a proposição é equivalente a dizer que $\Xi$ é finitamente gerado sobre $\Gamma$.

Pela Observação 1.4 sabemos que o semigrupo $\Gamma$ é finitamente gerado, ou seja, $\Gamma=\left\langle v_{0}, v_{1}, \ldots, v_{g}\right\rangle$. Seja $e=\operatorname{MDC}\left(v_{0}, v_{1}, \ldots, v_{g}\right)$, assim $\left\langle\frac{v_{0}}{e}, \frac{v_{1}}{e}, \ldots, \frac{v_{g}}{e}\right\rangle$ possui condutor, ou seja, existe $\mu$ tal que, para todo $\nu \geq \mu$ temos que $e \nu \in \Gamma$.

Considere $\Xi^{*}=\Xi \backslash\{0\}$ e defina

$$
\begin{gathered}
\lambda_{0}=\min \left\{\Xi^{*}\right\} \\
\lambda_{1}=\min \left\{\Xi^{*} \backslash\left\{\lambda_{0}+\Gamma\right\}\right\} \\
\lambda_{2}=\min \left\{\Xi^{*} \backslash \cup_{j=0}^{1}\left\{\lambda_{j}+\Gamma\right\}\right\}
\end{gathered}
$$




$$
\lambda_{i+1}=\min \left\{\Xi^{*} \backslash \cup_{j=0}^{i}\left\{\lambda_{j}+\Gamma\right\}\right\}
$$

Agora basta observar que existe $i$, tal que $\Xi^{*}=\cup_{j=0}^{i}\left\{\lambda_{j}+\Gamma\right\}$. Suponha que a seqüência acima seja infinita, assim, existem infinitos $\lambda_{i}$ que são congruentes entre si módulo $e$. Em particular, existe $j$ suficientemente grande, de modo que $\lambda_{i}-\lambda_{j} \geq e \mu$ e $\lambda_{i} \equiv \lambda_{j} \bmod (e)$, mas desta forma $\lambda_{j}=\lambda_{i}+\nu$ com $\nu \geq \mu$, o que implica $\lambda_{j} \in\left\{\lambda_{i}+\Gamma\right\}$ que é um absurdo.

Definição 2.3 Dizemos que uma diferencial $\omega \in \mathcal{O} d \mathcal{O}$ é exata, se existe $g \in \mathcal{O}$ tal que $\omega=d g$. Caso contrário, dizemos que $\omega$ é uma diferencial não exata (DNE).

OK-espaço vetorial constituído das diferenciais exatas será denotado por $d \mathcal{O}$.

Seja $\Gamma$ o semigrupo de valores associado à $C$ e $\mu$ seu condutor. Se $\omega \in$ $\mathcal{O} d \mathcal{O}$ é uma diferencial exata, então $v(\omega)+1 \in \Gamma$. Por outro lado, se $v(\omega)+1 \notin$ $\Gamma$, ou seja, $v(\omega)+1$ é uma lacuna de $\Gamma$, certamente $\omega$ é DNE. Além disso, se $v(\omega) \geq \mu-1$, então $\omega$ é uma diferencial exata.

Desse modo, ao invés de considerar $\Xi$, como na proposição anterior, utilizaremos $\Lambda=\{v(\omega)+1 ; \omega \in \mathcal{O} d \mathcal{O}\}$. Veja que $\Gamma \subseteq \Lambda$ e portanto, para todo inteiro $l \geq \mu$, temos que $l \in \Lambda$.

Definição 2.4 Seja $\Lambda$ como acima. O maior inteiro l tal que $l \notin \Lambda e ́$ chamado de lacuna limitante de $\Lambda$.

Note que $l \notin \Gamma$ el $l \leq \mu-1$.

Podemos melhorar o algoritmo para obter uma Base Standard para módulos no caso específico de $\mathcal{O} d \mathcal{O}$, levando-se em consideração alguns fatos.

Inicialmente observe que os $S$-processos mínimos de um par $(g, g) \in G \times G$ com $G \subseteq K[[\underline{X}]]$, apesar de não serem nulos, como já observamos, sempre se. reduzirão a zero, assim são triviais e não necessitam ser computados.

Seja $C$ é dada por

$$
\left\{\begin{array}{lll}
x_{1} & = & t^{v_{0}} \\
\vdots & \vdots & \vdots \\
x_{n} & = & p_{n}(t) .
\end{array}\right.
$$


Podemos iniciar o algoritmo para calcular uma Base Standard para o $\mathcal{O}$-módulo $\mathcal{O} d \mathcal{O}$ com o conjunto de geradores $\left\{d x_{1}, \ldots, d x_{n}\right\}$. No entanto, se $F=\left\{h_{i} ; i=0, \ldots, g\right\}$ é uma Base Standard Mínima para $\mathcal{O}$, então $d h_{i} \in \mathcal{O} d \mathcal{O}$ para todo $i=0, \ldots, g$ e $\left\{d h_{1}, \ldots, d h_{g}\right\}$ é também um conjuuto de geradores para $\mathcal{O} d \mathcal{O}$, podemos assim, iniciar o algoritmo com este conjunto, evitando assim, que efetuemos cálculos desnecessários.

Além dessas economias no algoritmo do Tcorema 1.5, podemos usar o conceito de lacuna linitante para eliminar alguns $S$-processos irrelevantes. De fato, se temos $\Lambda_{i}=\left\{v\left(F^{\alpha} \omega\right)+1 ; \omega \in G_{i}\right.$ e $\left.\alpha \in \mathbb{N}^{\sharp F}\right\}$ em um passo $i$ do algoritmo do Teorema 1.5 e $l_{i}$ é sua lacuna limitante, então obviamente $l \leq l_{i}$. Como todo $S$-processo mínimo de altura maior on igual a $t^{l_{i}}$ possui uma redução final zero, módulo $\left(G_{i}, F\right)$, podemos excluir tais $S$-processos mínimos do conjunto $S$.

Temos assim, como corolário do Teorema 1.5 e das considerações acima, o seguinte algoritmo para calcular uma Base Standard para $\mathcal{O} d \mathcal{O}$, apartir de uma Base Standard Mínima $F$ de $\mathcal{O}$.

\section{Algoritmo 2.2}

$D A D O: F$;

DEFINA: $G_{0}:=\{\}$

$G:=\{d h ; h \in F\}$;

ENQUANTO $G \neq G_{0}$ FAÇA

$\Lambda:=\left\{v\left(F^{\alpha} \omega\right)+1 ; \omega \in G e \alpha \in \mathbb{N}^{\sharp F}\right\} ;$

$l:=$ lacuna limitante de $\Lambda$;

$S:=\{s ; s$ é S-processo mínimo não trivial de $G$ com $v(h t,(s))<l$, não computado no passo anterior $\}$;

$R:=\left\{r ; s \stackrel{G_{F+}}{\longrightarrow} r\right.$ er $\left.\neq 0\right\}$;

$G_{0}:=G$;

$G:=G \cup R$

SAIDDA: $G, \Lambda, l$.

Observação 2.4 1. No algoritmo acima, são computadas apenas DNE. As DNE pertencentes a uma Base. Standard Mínima de. OdO serão chamadas de diferenciais não exatas minïmais, ou simplesmente DNEM.

2. Como mencionamos no início desta seção, o $\mathcal{O}$-submódulo de torção $T$ de $\mathcal{O} d \mathcal{O}$, representa um importante objeto para o estudo de uma curva 
algebróide irredutivel $G$. Em particular, quando $C$ é uma interseção completa, Berger, em [Ber], mostra que o comprimento do submódulo de torção $l(T)$ pode ser calculado por

$$
l(T)=\mu-\sharp(\Lambda \backslash \Gamma),
$$

ou equivalentemente

$$
l(T)=\frac{\mu}{2}+\sharp(\mathbb{N} \backslash \Lambda)
$$

onde $\mu$ é o condutor do semigrupo $\Gamma$ associado à $C$.

3. Do mesmo modo que no algoritmo para Base Standard de álgebras, é conveniente estabelecer uma maneira de efetuar o processo de redução. Podemos, por exemplo, ordenar os elementos do conjunto $G$ segundo suas ordens, ou pela ordem com que forem computados se dois ou mais deles tiverem mesma ordem, e se for possivel reduzir um elemento de $K[[t]]$ por $F^{\alpha} g$ e $F^{\beta} h$, escolhemos efetuar a redução por $F^{\alpha} g$ se $v(g)<$ $v(h)$ ou se $F^{\alpha}$ for a escolha, segundo a maneira fixada para a redução como álgebras, quando $v(g)=v(h)$.

Definição 2.5 Considerando as mesmas notações anteriores, chamamos os elementos de $\Lambda \backslash \Gamma$ de lacunas especiais de $C$.

Exemplo 2.4 1. Seja

$$
C:\left\{\begin{array}{l}
x=t^{8} \\
y=t^{10}+t^{13} \\
z=t^{12}+t^{15}
\end{array}\right.
$$

Pelo item 3) do Exemplo 2.1, temos que. $F=\left\{x=t^{8}, y=t^{10}+t^{13}, z=\right.$ $\left.t^{12}+t^{15}, u=t^{23}+t^{26}, w=2 t^{27}+t^{30}\right\}$ é uma Base Standard Mínima para $\mathcal{O}$ e $\Gamma=\langle 8,10,12,23,27\rangle$ é o semigrupo de valores de $C$, cujo condutor é $\mu=30$.

Note que $\Gamma$ não é simétrico, assim $C$ não é Gorenstein, conseqüentemente não é uma interseção completa.

Apliquemos o algoritmo anterior para $G=\{d x, d y, d z, d u, d w\}$. 
Temos quel $l=\mu-1=29$ é a lacuna limitante nesse passo e o conjunto dos $S$-processos mínimos de $G$ a serem considerados é

$S:=\{4 x d y-5 y d x, 4 y d y-5 z d x, 6 y d y-5 x d z, 2 x d z-3 z d x$,

$$
\left.5 y d z-6 z d y, 2 z d z-3 x^{2} d x, 2 x^{2} d z-3 y^{2} d x\right\} \text {. }
$$

Calculando as reduções finais módulo $(G, F)$, temos:

$$
\begin{gathered}
4 x d y-5 y d x=\Omega_{1} \Rightarrow \psi\left(\Omega_{1}\right)=12 t^{20} \\
5 y d z-6 z d y=\Omega_{2} \Rightarrow \psi\left(\Omega_{2}\right)=-3 t^{24}-3 t^{27} .
\end{gathered}
$$

Como $v\left(\Omega_{1}\right)+1=21, v\left(\Omega_{2}\right)+1=25$ e $v\left(x \Omega_{1}\right)+1=29$ vemos que $a$ lacuna limitante passa a ser $l=19$, o que permite descartar os demais $S$-processos.

Uma simples análise mostra que não há $S$-processos mínimos a serem. considerados no próximo passo, assim o algoritmo finaliza com a Base Standard Mínima de O-módulos

$$
G=\left\{d x, d y, d z, d u, d w, \Omega_{1}, \Omega_{2}\right\}
$$

para $\mathcal{O} d \mathcal{O}$

2. Considere

$$
C:\left\{\begin{array}{l}
x=t^{6} \\
y=t^{8}+2 t^{9} \\
z=t^{10}+t^{11}
\end{array}\right.
$$

Pelo Exemplo 2.3, temos que $\Gamma=\langle 6,8,10,17,19\rangle$ é o semigrupo de. valores de $C, \mu=22$ é. seu condutor e $F=\{x, y, z, w, u\}$, com $w=$ $x z-y^{2}=-3 t^{17}-4 t^{18}$ e $u=y+z-x^{3}=3 t^{19}+2 t^{20}$ é uma Base Standard Mínima para $\mathcal{O}$.

Aplicando o algoritmo anterior para $G=\{d x, d y, d z, d w, d u\}$ e usando que $l=\mu-1=21$ é a lacuna limitante, temos que os $S$-processos a serem analisados no primeiro passo são:

$S=\left\{3 x d y-4 y d x, 3 y d y-4 x^{2} d x, 4 x d z-5 y d y\right.$ 


$$
\begin{aligned}
& 3 x d z-5 z d x, 3 z d y-4 x^{2} d x, 4 y d z-5 z d y \\
& \left.3 y d z-5 x^{2} d x, 3 z d z-5 x y d x, 4 z d z-5 x^{2} d y\right\}
\end{aligned}
$$

Como

$$
3 x d y-4 y d x=\Omega \Rightarrow \psi(\Omega)=6 t^{14},
$$

temos que $v(\Omega)+1=15$ e $v(x \Omega)+1=21$, assim a lacuna limitante pode ser considerada como $l=13$, indicando assim, que os demais $S$-processos se reduzem a zero e que não há $S$-processos que mereçam. análise no próximo passo, o que finaliza o algoritmo.

Apesar de $\Gamma$ não ser de interseção completa, a curva $C$ o é, como observamos no Exemplo 2.3.

Portanto,

$$
l(T)=\mu-\sharp(\Lambda \backslash \Gamma)=22-\sharp\{15,21\}=20
$$

3. Considere

$$
C:\left\{\begin{array}{l}
x=t^{8} \\
y=t^{12}+t^{13}
\end{array}\right.
$$

Usando a Proposição 2.1, temos que $F=\{x, y, z\}$ com $z=y^{2}-x^{3}=$ $2 t^{25}+t^{26}$ é uma Base Standard Mínima para $\mathcal{O}, \Gamma=\langle 8,12,25\rangle$ é o semigrupo associado à $C$ e $\mu=80$.

Aplicando o algoritmo anterior para $G=\{d x, d y, d z\}$, temos:

\section{Passo 1:}

A lacuna limitante nesse passo é $l=79$ e os $S$-processos mínimos a serem considerados são:

$S:=\left\{3 y d x-2 x d y, 8 y d y-12 x^{2} d x, 8 x d z-25 z d x\right.$

$$
\left.12 y d z-25 z d y, 25 x^{2} z d x-8 y^{2} d z, 25 y z d y-12 x^{3} d z\right\} \text {. }
$$

Calculando a redução final dos elementos de $S$ módulo $(G, F)$, temos:

- $3 y d x-2 x d y=\Omega_{1} \Rightarrow \psi\left(\Omega_{1}\right)=-2 t^{20}$.

- $8 y d y-12 x^{2} d x \stackrel{-4 d z}{\longrightarrow} 0$.

- $8 x d z-25 z d x=\Omega_{2} \Rightarrow \psi\left(\Omega_{2}\right)=8 t^{33}$. 
- $12 y d z-25 z d y=\Omega_{3} \Rightarrow \psi\left(\Omega_{3}\right)=-38 t^{37}-13 t^{38}$.

- $25 x^{2} z d x-8 y^{2} d z \stackrel{\frac{202}{25} z d z}{\longrightarrow} \Omega_{4} \Rightarrow \psi\left(\Omega_{4}\right)=\frac{204}{25} t^{50}+\frac{52}{25} t^{51}$.

- $25 y z d y-12 x^{3} d z \stackrel{-\frac{619}{50} z d z}{\longrightarrow} \frac{3}{2} \Omega_{4} \Rightarrow \psi\left(\frac{3}{2} \Omega_{4}\right)=\frac{306}{25} t^{50}+\frac{78}{25} t^{51}$.

\section{Passo 2:}

Uma vez que $v\left(z \Omega_{2}\right)+1=59, v\left(z \Omega_{3}\right)+1=63, v\left(x z \Omega_{1}\right)+1=$ 67, $v\left(z^{2} \Omega_{1}\right)+1=71$ e $v\left(x z^{2} \Omega_{1}\right)+1=79$, a lacuna limitante passa $a$ $\operatorname{ser} l=55$ e $G:=\left\{d x, d y, d z, \Omega_{1}, \Omega_{2}, \Omega_{3}, \Omega_{4}\right\}$.

Os S-processos mínimos de $G$ que não foram analisados no Passo 1 e. cuja altura é menor que $t^{55}$, são:

$$
\begin{aligned}
S=\{ & z d x+8 y \Omega_{1}, x d z+25 y \Omega_{1}, z d y+12 x^{2} \Omega_{1}, y d z+25 x^{2} \Omega_{1}, \\
& y z d x+8 x^{3} \Omega_{1}, x z d y+12 y^{2} \Omega_{1}, 2 z \Omega_{1}+y \Omega_{2}, 4 x \Omega_{3}-38 z \Omega_{1}, \\
& \left.19 y \Omega_{2}+4 x \Omega_{3}, 4 y \Omega_{3}+19 x^{2} \Omega_{2}\right\} .
\end{aligned}
$$

Efetuando a redução final módulo $(G, F)$, obtemos

- $z d x+8 y \Omega_{1} \stackrel{\Omega_{2}}{\longrightarrow} 0$

- $x d z+25 y \Omega_{1} \stackrel{3 \Omega_{2}}{\longrightarrow} 0$,

- $z d y+12 x^{2} \Omega_{1} \stackrel{\Omega_{3}}{\longrightarrow} 0$

- $y d z+25 x^{2} \Omega_{1} \stackrel{2 \Omega_{3}}{\longrightarrow} 0$

- $y z d x+8 x^{3} \Omega_{1} \stackrel{6 z \Omega_{1}}{\longrightarrow} \Omega_{5} \Rightarrow \psi\left(\Omega_{5}\right)=-4 t^{46}$,

- $x z d y+12 y^{2} \Omega_{1} \stackrel{-\frac{5}{2} z \Omega_{1}}{\longrightarrow} \frac{3}{2} \Omega_{5} \Rightarrow \psi\left(\frac{3}{2} \Omega_{5}\right)=-6 t^{46}$.

Temos que $v\left(z \Omega_{1}\right)+1=46, v\left(\Omega_{5}\right)+1=47, v\left(x z \Omega_{1}\right)+1=54 e$ $v\left(x \Omega_{5}\right)+1=55$ assim, a lacuna limitante no próximo passo será $l=43$, conseqüentemente, podemos dispensar a análise dos demais $S$-processos e passamos ao próximo passo.

\section{Passo 3:}

Temos $G:=\left\{d x, d y, d z, \Omega_{1}, \Omega_{2}, \Omega_{3}, \Omega_{4}, \Omega_{5}\right\}$. Como neste passo devemos calcular os $S$-processos que envolvem $\Omega_{5}$ e $v\left(\Omega_{5}\right)+1$ supera a lacuna limitante o algoritmo finaliza. A saber, $G$ é uma Base Standard Mínima para $\mathcal{O} d \mathcal{O}$. 
Como $C$ é uma curva algebróide irredutivel plana, portanto de interseção completa, temos que

$$
\begin{aligned}
l(T) & =\mu-\sharp(\Lambda \backslash \Gamma)= \\
& =80-\sharp\{21,29,34,38,42,46,47,51,54,55,59,63,67,71,79\}= \\
& =80-15=65 .
\end{aligned}
$$

O exemplo anterior foi tratado por Azevedo, com outras técnicas mais rudimentares, em sua tese [Az] pag. 79. No entanto, a existência de uma DNE com ordem igual a de $\Omega_{4}$ não foi detectada, deixando o exemplo incompleto.

Definição 2.6 Duas curvas algebróides irredutíveis equisingulares são equidiferenciáveis, se elas possuem o mesmo conjunto $\Lambda=\{v(\Omega)+1 ; \Omega \in \mathcal{O} d \mathcal{O}\}$.

Veremos no próximo capítulo que o conjunto $\Lambda$, juntamente com $\Gamma$, são muito importantes para o estudo das curvas algebróides irredutíveis.

Apesar de $\Gamma \subseteq \Lambda$, é falso afirmar que curvas algebróides irredutíveis que possuem mesmo conjunto $\Lambda$ são equidiferenciáveis, pois podemos ter curvas que não são equisingulares e no entanto, possuem mesmo conjunto $\Lambda$, como mostra o exemplo abaixo.

Exemplo 2.5 Sejam as curvas dadas pelas parametrizações:

$$
C_{1}:\left\{\begin{array}{l}
x=t^{6} \\
y=t^{14}+t^{17} \quad \text { e } C_{2}:\left\{\begin{array}{l}
x=t^{6} \\
z=t^{39}
\end{array}=t^{14}+t^{33}\right. \\
z=t^{23}
\end{array}\right.
$$

Aplicando o Algoritmo 2.1 obtemos os semigrupos

$$
\Gamma_{1}=\langle 6,14,39\rangle \text { e } \Gamma_{2}=\langle 6,14,23\rangle .
$$

A aplicação do Algoritmo 2.2 indica que uma Base Standard Mínima para os módulos de diferenciais das curvas acima é $G_{1}=G_{2}=\{d x, d y, d z, \Omega\}$, onde

$$
\Omega=x d y-\frac{14}{6} y d x=\left\{\begin{array}{l}
3 t^{22} \text { para } C_{1} \\
19 t^{38} \text { para } C_{2} .
\end{array}\right.
$$

Desse modo, $\Lambda_{1}=\Lambda_{2}=\{0,6,12,14,18,20,23,24,26,28,29,30,32,34, \ldots\}$, embora $\Gamma_{1} \neq \Gamma_{2}$. 


\subsubsection{Curvas de Interseção Completa}

No restante desta seção, $C$ é uma curva algebróide irredutível de interseção completa, oı seja, $C$ é dada por um conjurıto de equações $f_{1}, \ldots, f_{n-1} \in$ $\mathcal{M}_{X}^{*} \subset K\left[\left[X_{1}, \ldots, X_{n}\right]\right]$.

Seja $v\left(X_{1}\right)=v_{0}=\operatorname{mult}(C)$. Em $\mathcal{O}$, temos que $\sum_{j=1}^{n} f_{i_{x_{j}}} d x_{j}=0$ para todo $i=1, \ldots, n-1$, ou equivalentemente,

$$
M_{1} \cdot\left(\begin{array}{c}
d x_{2} \\
\vdots \\
d x_{n}
\end{array}\right)=-\left(\begin{array}{c}
f_{1_{x_{1}}} \\
\vdots \\
f_{n-1_{x_{1}}}
\end{array}\right) \cdot d x_{1}
$$

onde

$$
M_{1}=\left(\begin{array}{ccc}
f_{1_{x_{2}}} & \cdots & f_{1_{x_{n}}} \\
\vdots & \ddots & \vdots \\
f_{n-1 x_{2}} & \cdots & f_{n-1} x_{n}
\end{array}\right) .
$$

Carbonme, em [Car] pag. 376, mostra que $\left|M_{1}\right|=\operatorname{det}\left(M_{1}\right) \in \mathcal{O}^{*}$ e $\left|M_{i}\right|=$ $\operatorname{det}\left(M_{i}\right) \in \mathcal{O}^{*}$, onde

$$
M_{i}=\left(\begin{array}{ccccccc}
f_{1 x_{2}} & \cdots & f_{1 x_{i-1}} & -f_{1 x_{1}} & f_{1 x_{i+1}} & \cdots & f_{1 x_{n}} \\
\vdots & \ddots & \vdots & \vdots & \vdots & \ddots & \vdots \\
f_{n-1 x_{2}} & \cdots & f_{n-1 x_{i-1}} & -f_{n-1 x_{1}} & f_{n-1 x_{i+1}} & \cdots & f_{n-1 x_{n}}
\end{array}\right)
$$

para todo $i=2, \ldots, n$.

Desse modo, em $\mathcal{O} d \mathcal{O} \otimes_{\mathcal{O}} \mathcal{K}$, onde $\mathcal{K}$ é o corpo de frações de $\mathcal{O}$, temos

$$
d x_{i}=\frac{\left|M_{i}\right|}{\left|M_{1}\right|} d x_{1}
$$

para todo $i=1, \ldots, n$.

Observação 2.5 Herzog e Kunz, em [HK], mostram que para uma curva de interseção completa $C$ tem-se

$$
v\left(\left|M_{1}\right|\right)=\mu+v_{0}-1
$$

onde $\mu$ é o condutor do semigrupo associado à $C$ e v $v_{0}$ é a multiplicidade da curva. 
Assim, denotando por $x_{i}^{\prime}=\psi\left(d x_{i}\right)$ para $i=1, \ldots, n$, temos

$$
v\left(\frac{\left|M_{i}\right|}{x_{i}^{\prime}}\right)=\mu \text {. }
$$

No caso de curvas planas, isto foi mostrado por Azevedo, em [Az] Prop. 5 pag. 17, e por Zariski, em. [Z2] pag. 11.

Utilizando a relação $(2.5)$, que $v\left(x_{1}^{\prime}\right)=v_{0}-1$ e que

$$
v\left(\frac{f}{g}\right)=\operatorname{dim}_{K} \frac{K[[\underline{X}]]}{\left\langle f_{1}, \ldots, f_{n-1}, f\right\rangle}-\operatorname{dim}_{K} \frac{K[[\underline{X}]]}{\left\langle f_{1}, \ldots, f_{n-1}, g\right\rangle}
$$

para hipersuperfícies $f, g \in K[[\underline{X}]]$, podemos aplicar o algoritmo para obter uma Base Standard de $\mathcal{O}$-módulos para $\mathcal{O} d \mathcal{O}$, na verdade para $\psi(\mathcal{O} d \mathcal{O})$, mesmo quando a curva $C$ for dada por meio de equações $f_{1}, \ldots, f_{n-1} \in$ $\mathcal{M}_{\underline{X}}^{*} \subset K[[\underline{X}]]$.

Exemplo 2.6 Seja C a curva algebróide irredutível plana dada por

$$
f(X, Y)=Y^{3}+X^{5} Y+X^{7} .
$$

Uma vez que $\operatorname{dim}_{K} \frac{K[\mid X, Y]]}{\langle f, X\rangle}=3$ edim. $\frac{K[\mid X, Y]]}{\langle f, Y\rangle}=7$, temos que $B=\{X, Y\}$ é uma Base Standard Mínima para $\mathcal{O}, \Gamma=\langle 3,7\rangle$ é o semigrupo associado à C e $\mu=12$ é seu condutor.

Como $C$ é plana e portanto de interseção completa, temos que

$$
\left|M_{1}\right|=f_{y} \text { e }\left|M_{2}\right|=-f_{x} .
$$

Vamos aplicar o algoritmo para obter uma Base Standard de $\psi(\mathcal{O} d \mathcal{O})$. Inicialmente temos $G=\{d x, d y\}$ e a lacuna limitante é $l=\mu-1=11$.

$O$ único $S$-processo minimo a ser considerado é da forma $S=x d y-$ $a_{1} y d x$.

Usando que $d y=-\frac{f_{x}}{f_{y}} d x$, temos que

$$
S=\left(-x \frac{f_{x}}{f_{y}}-a_{1} y\right) d x=\left(-x f_{x}-a_{1} y f_{y}\right) \frac{d x}{f_{y}} .
$$

Como $v(g)=\operatorname{dim}_{K} \frac{\dot{K}[[X, Y]]}{\langle(f, g)}=\operatorname{ord}_{X}\left(R_{Y}(f, g)\right)$ para $g \in K[[X, Y]]$, podemos determinar o valor de $a_{1}$ de modo que $S$, seja de fato, um $S$-processo, ou seja, $v(S)>v(x d y)=v(y d x)=9$. 
Uma vez que

$$
R_{Y}\left(f,-X f_{X}-a_{1} Y f_{Y}\right)=\left(3 a_{1}-7\right)^{3} X^{21}+\left(a_{1}-2\right)\left(2 a_{1}-5\right)^{2} X^{22},
$$

a única escolha para $a_{1}$ de modo a tornar $S$ um $S$-processo é $a_{1}=\frac{7}{3}$, i.e., $S=x d y-\frac{7}{3} y d x$ e dessa forma

$$
v(S)=\operatorname{ord}_{X}\left(R_{Y}\left(f,-X f_{X}-\frac{7}{3} Y f_{Y}\right)\right)+v\left(\frac{d x}{f_{y}}\right)=22-12=10 .
$$

Assim temos, $\Omega=x d y-\frac{7}{3} y d x, \operatorname{com} v(\Omega)+1=11$.

Uma vez que no próximo passo a lacuna limitante é $l=8$, vemos que não existem. $S$-processos a serem considerados. Portanto, o algoritmo finaliza. com a Base Standard Minima $G=\{d x, d y, \Omega\}$ para $\psi(\mathcal{O} d \mathcal{O})$.

Além disso, como $C$ é de interseção completa, temos que

$$
l(T)=\mu-\sharp(\Lambda \backslash \Gamma)=12-1=11 .
$$

Definição 2.7 SejaC uma curva algebróide irredutivel dada por $f_{1}, \ldots, f_{n-1}$ $\in \mathcal{M}_{\underline{X}}^{*} \subset K\left[\left[X_{1}, \ldots, X_{n}\right]\right]$ e $\left|M_{i}\right|$ para $i=1, \ldots, n$ como definidos em (2.3) $e$ (2.4).

$O$ ideal jacobiano de $C$ é o ideal $\mathcal{J}$ de $\mathcal{O}$ gerado por $\left|M_{i}\right|$ para $i=1, \ldots, n$, i.e.,

$$
\mathcal{J}=\left\langle\left|M_{1}\right|, \ldots,\left|M_{n}\right|\right\rangle \mathcal{O} .
$$

$A$ codimensão de $\mathcal{J}$ em $\mathcal{O}$ é chamada de número de Tjurina de $C$, ou seja,

$$
\tau:=\operatorname{dim}_{K} \frac{K\left[\left[X_{1}, \ldots, X_{n}\right]\right]}{\left\langle f_{1}, \ldots, f_{n-1},\left|M_{1}\right|, \ldots,\left|M_{n}\right|\right\rangle}=\operatorname{dim}_{K} \frac{\mathcal{O}}{\mathcal{J}}
$$

Pela Observação 2.5, temos que a multiplicidade $e(\mathcal{J})$ do ideal $\mathcal{J}$, ou seja, a menor ordem de um elemento não nulo de $\mathcal{J}$, é

$$
e(\mathcal{J})=\mu+v_{0}-1
$$

onde $v_{0}$ é multipliciade de $C$ e $\mu$ é o condutor do semigrupo associado à $C$.

Sejam $\varphi$ e $\psi$ as aplicaçôes, definidas em (2.1) e (2.2), dadas respectivamente por

$$
\begin{array}{cccc}
\varphi: & \mathcal{O} & \longrightarrow & K[[t]] \\
g & \mapsto & g\left(p_{1}(t), \ldots, p_{n}(t)\right) \\
\psi: & \mathcal{O} d \mathcal{O} & \longrightarrow & K[[t]] \\
& \sum_{i=1}^{n} g_{i} d x_{i} & \mapsto & \sum_{i=1}^{n} \varphi\left(g_{i}\right) x_{i}^{\prime}
\end{array}
$$


onde $g, g_{1}, \ldots ; g_{n} \in \mathcal{O}, x_{i}=p_{i}(t)$ para $i=1, \ldots, n$ é uma parametrização para $C$ com $x_{1}=t^{v_{0}}$ onde $v_{0}=\operatorname{mult}(C)$ e $x_{i}^{\prime}=\frac{d p_{i}(t)}{d t}$.

Uma vez que $\mathcal{J}$ e $\mathcal{O} d \mathcal{O}$ são $\mathcal{O}$-módulos gerados por $\left\{\left|M_{1}\right|, \ldots,\left|M_{n}\right|\right\}$ e $\left\{d x_{1}, \ldots, d x_{n}\right\}$ respectivamente, temos que $\varphi(\mathcal{J})$ e $\psi(\mathcal{O} d \mathcal{O})$ são $\varphi(\mathcal{O})$-módulos gerados por $\left\{\varphi\left(\left|M_{1}\right|\right), \ldots, \varphi\left(\left|M_{n}\right|\right)\right\}$, respectivamenle por $\left\{x_{1}^{\prime}, \ldots, x_{n}^{\prime}\right\}$.

Considere o epimorfismo de $\varphi(\mathcal{O})$-módulos

$$
\begin{array}{cccc}
\phi: & \varphi(\mathcal{J}) & \longrightarrow & \psi(\mathcal{O} d \mathcal{O}) \\
\varphi\left(\left|M_{i}\right|\right) & \mapsto & x_{i}^{\prime}
\end{array}
$$

para todo $i=1, \ldots, n$.

Lema 2.1 O epimorfismo $\phi$ está bem definido e é um isomorfismo de $\varphi(\mathcal{O})$ módulos.

Dem.: Mostremos que $\phi$ está bem definido.

Seja $\sum_{i=1}^{n} \varphi\left(g_{i}\right) \varphi\left(\left|M_{i}\right|\right)=0$ com $g_{i} \in \mathcal{O}$. Uma vez que em $\mathcal{O} d \mathcal{O} \otimes_{\mathcal{O}} \mathcal{K}$ temos (2.5), i.e.,

$$
d x_{i}=\frac{\left|M_{i}\right|}{\left|M_{1}\right|} d x_{1},
$$

para todo $i=1, \ldots, n$. $\operatorname{Em} K((t))$ temos

$$
\varphi\left(\left|M_{i}\right|\right)=\varphi\left(\left|M_{1}\right|\right) \frac{x_{i}^{\prime}}{x_{1}^{\prime}}
$$

para todo $i=1, \ldots, n$.

Desse modo

$$
\begin{aligned}
0 & =\sum_{i=1}^{n} \varphi\left(g_{i}\right) \varphi\left(\left|M_{i}\right|\right)= \\
& =\varphi\left(g_{1}\right) \varphi\left(\left|M_{1}\right|\right)+\sum_{i=2}^{n} \varphi\left(g_{i}\right) \varphi\left(\left|M_{1}\right|\right) \frac{x_{i}^{\prime}}{x_{1}}= \\
& =\frac{\varphi\left(\left|M_{1}\right|\right)}{x_{1}^{\prime}} \sum_{i=1}^{n} \varphi\left(g_{i}\right) x_{i}^{\prime} .
\end{aligned}
$$

Portanto, $\sum_{i=1}^{n} \varphi\left(g_{i}\right) x_{i}^{\prime}=0$, mostrando que $\phi$ está bem definido.

De modo análogo, mostra-se que $\phi$ é injetor. 
$O$ isomorfismo $\phi$ possui interessantes propriedades. Por exemplo, a proposição abaixo mostra que podemos, por meio de $\phi$, relacionar uma Base St,andard Mínima de $\varphi(\mathcal{J})$ com uma Base Standard Mínima de $\psi(\mathcal{O} d \mathcal{O})$. Além disso, $\phi$ preserva a ordenação dada pela valorização discreta $v$ de $\overline{\mathcal{O}}=K[[t]]$.

Proposição 2.3 Seja $\phi$ o isomorfismo de $\varphi(\mathcal{O})$-módulos definido em (2.6). Temos que:

1. $v(g)=v(\phi(g))+\mu$, para todo $g \in \varphi(\mathcal{J})$.

2. Se $G=\left\{\omega_{1}, \ldots, \omega_{r}\right\}$ é uma Base Standard Mínima de $\varphi(\mathcal{O})$-módulos para $\psi(\mathcal{O} d \mathcal{O})$, então $\phi^{-1}(G)$ é uma Base Standard Minima de $\varphi(\mathcal{O})$ módulos para $\varphi(\mathcal{J})$.

Dem.: Uma vez que

$$
d x_{i}=\frac{\left|M_{i}\right|}{\left|M_{1}\right|} d x_{1}
$$

para todo $i=1, \ldots, n$ em $\mathcal{O} d \mathcal{O} \otimes_{\mathcal{O}} \mathcal{K}$, um elemento $g . \in \varphi(\mathcal{J})$ pode ser escrito como

$$
g=\sum_{i=1}^{n} h_{i} \varphi\left(\left|M_{i}\right|\right)=\frac{\varphi\left(\left|M_{1}\right|\right)}{x_{1}^{\prime}} \sum_{i=1}^{n} h_{i} x_{i}^{\prime}
$$

onde $h_{i} \in \varphi(\mathcal{O})$ para $i=1, \ldots, n$.

Assim, pela Observação 2.5 temos

$$
v(g)=v\left(\sum_{i=1}^{n} h_{i} \varphi\left(\left|M_{i}\right|\right)\right)=v\left(\sum_{i=1}^{n} h_{i} x_{i}^{\prime}\right)+\mu=v(\phi(g))+\mu .
$$

Scjam $F$ uma Base Standard Mínima de álgebras para $\varphi(\mathcal{O})$ : $G$ uma Base Standard Mínima de $\varphi(\mathcal{O})$-módulos para $\psi(\mathcal{O} d \mathcal{O})$. Estabelecida uma maneira de efetuar a redução por $F$ e por $(G, F)$, temos que cada elemento de $\psi(\mathcal{O} d \mathcal{O})$ tem uma representação única segundo essa maneira fixada.

Tomando um $S$-processo, como $\varphi(\mathcal{O})$-módulo, de um par de elemen$\operatorname{tos}\left(\phi^{-1}\left(\omega_{1}\right), \phi^{-1}\left(\omega_{2}\right)\right) \in \phi^{-1}(G) \times \phi^{-1}(G)$, digamos $S=F^{\alpha} \phi^{-1}\left(\omega_{1}\right)-$ $a F^{\beta} \phi^{-1}\left(\omega_{2}\right)$, vemos que $S$ possui uma redução final zero módulo $\left(\phi^{-1}(G), F\right)$.

De fato, escrevendo $S=\phi^{-1}\left(F^{\alpha} \omega_{1}-a F^{\beta} \omega_{2}\right)$ e usando o fato de que $G$ é uma Base Standard de módulos para $\psi(\mathcal{O} d \mathcal{O})$, pelo processo de redução, temos que

$$
F^{\alpha} \omega_{1}-a F^{\beta} \omega_{2}=\sum_{i=1}^{\sharp G}\left(\sum_{\gamma \in \Upsilon_{i}} b_{\gamma} F^{\gamma}\right) \omega_{i}
$$


com $b_{\gamma} \in K$ e $\Upsilon \subset \mathbb{N}^{\sharp F}$. Mas isso induz uma redução final de $S$, módulo $\left(\phi^{-1}(G), F\right)$, de forma a podermos escrever

$$
S=\sum_{i=1}^{\sharp G}\left(\sum_{\gamma \in \Upsilon_{i}} b_{\gamma} F^{\gamma}\right) \phi^{-1}\left(\omega_{i}\right) .
$$

Portanto, $\phi^{-1}(G)$ é uma Base Standard de módulos para $\mathcal{J}$ e como as ordens dos elementos de $\phi^{-1}(G)$ são todas distintas, pois $G$ é Base Standard Mínima, segue que a Base Standard $\phi^{-1}(G)$ também o é.

Observe que se $h \in K[[t]]$ é tal que $v(h) \geq \mu-1$, então $h \in \psi(\mathcal{O} d \mathcal{O})$, conseqüentemente, qualquer elemento de $\varphi(\mathcal{O})$ com ordem maior ou igual a $2 \mu-1$ pertence a $\varphi(\mathcal{J})$.

A proposição anterior nos dá como corolário um resultado obtito por Zariski, em [Z1], no caso de curvas planas e generalizado por Pinkham, em [Pi] Chap. III 10.4 para curvas Gorenstein.

Corolário 2.1 Se Cé uma curva algebróide irredutivel de interseção completa, então $\tau=l(T)$, onde $T \subset \mathcal{O} d \mathcal{O}$ é o $\mathcal{O}$-submódulo de torção.

Dem.: Da Definição 2.7 de $\tau$, temos que $\tau=\sharp(\Gamma-v(\mathcal{J}))$, basta assim, calcularmos quantos elementos de $\Gamma$ menores que $2 \mu,-1$ não pertencem a $v(\mathcal{J})$. Uma vez que temos $\frac{\mu}{2}$ elementos até $\mu$ que estão em $\Gamma \backslash v(\mathcal{J})$, pois o menor elemento não nulo de $v(\mathcal{J})$ é $\mu+v_{0}-1$, resta calcular quantos elementos $\gamma \in \Gamma \backslash v(\mathcal{J})$ são da forma $\mu \leq \gamma<2 \mu-1$.

Pelo teorema anterior, para cada elemento $\lambda \in \Lambda$ obtemos um elemento em $v(\mathcal{J})$ da forma $\lambda+\mu$. Como até $\mu$, temos $\frac{\mu}{2}+\sharp(\Lambda \backslash \Gamma)$ tais elementos, no intervalo $\left[\mu, 2 \mu-1\left[\right.\right.$ teremos $\mu-\left(\frac{\mu}{2}+\sharp(\Lambda \backslash \Gamma)\right)$ elementos em $\Gamma \backslash v(\mathcal{J})$. Portanto,

$$
\tau=\sharp(\Gamma-v(\mathcal{J}))=\frac{\mu}{2}+\mu-\left(\frac{\mu}{2}+\sharp(\Lambda \backslash \Gamma)\right)=\mu-\sharp(\Lambda \backslash \Gamma)=l(T),
$$

onde a última igualdade é garantida pela Observação 2.4 . 
Exemplo 2.7 Seja

$$
C:\left\{\begin{array}{l}
x=t^{8} \\
y=t^{12}+t^{13},
\end{array}\right.
$$

cuja equação cartesiana é dada por $f(X, Y)=\left(Y^{2}-X^{3}\right)^{4}-8 X^{8} Y\left(Y^{2}+\right.$ $\left.X^{3}\right)-X^{13}$.

De acordo com o item 3 do Exemplo 2.4, $\mathcal{O}$ tem uma Base Standard Mínima dada por $F=\{x, y, z\}$ com $z=y^{2}-x^{3}$ e $\mathcal{O} d \mathcal{O}$ possui uma Base. Standard Mínima dada por $G=\left\{d x, d y, d z, \Omega_{1}, \Omega_{2}, \Omega_{3}, \Omega_{4}, \Omega_{5}\right\}$, onde.:

$$
\begin{gathered}
\Omega_{1}=3 y d x-2 x d y, \\
\Omega_{2}=8 x d z-25 z d x, \\
\Omega_{3}=12 y d z-25 z d y, \\
\Omega_{4}=25 x^{2} z d x-8 y^{2} d z+\frac{202}{25} z d z, \\
\Omega_{5}=y z d x+8 x^{3} \Omega_{1}-6 z \Omega_{1} .
\end{gathered}
$$

Assim, uma Base Standard Mínima para $\mathcal{J}$ é dada por

$$
\varphi^{-1}(G)=\left\{f_{Y}, f_{X}, f_{0}, f_{1}, f_{2}, f_{3}, f_{4}, f_{5}\right\}
$$

onde:

$$
\begin{gathered}
f_{0}=2 y f_{X}+3 x^{2} f_{Y} \\
f_{1}=3 y f_{Y}+2 x f_{X} \\
f_{2}=8 x f_{0}+25 z f_{Y} \\
f_{3}=12 y f_{0}-25 z f_{X} \\
f_{4}=-25 x^{2} z f_{Y}-8 y^{2} f_{0}+\frac{202}{25} z f_{0}, \\
f_{5}=-y z f_{Y}+8 x^{3} f_{1}-6 z f_{1} .
\end{gathered}
$$




\section{Capítulo 3}

\section{Equivalência de Curvas}

Neste capítulo, passaremos a uma das questões mais importantes da teoria, que permanece aberta, a classificação das curvas algebróides irredutíveis.

$\mathrm{Na}$ primeira seção, introduzimos a relação de equivalência de curvas algebróides irredutíveis, bem como invariantes aritméticos com respeito a essa relação. Alguns desses invariantes não dispunham de métodos sistemáticos para serem obtidos, conduzindo a alguns erros ou a resultados incompletos. No entanto, com as técnicas apresentadas nos capítulos anteriores, tais invariantes podem ser obtidos por algoritmos que são implementáveis.

A segunda seção se restringe ao caso das curvas algebróides irredutíveis planas, as quais denominamos simplesmente de ramos. Introduzimos o diagrama de lacunas especiais de um ramo e obtemos alguns refinamentos para o algoritmo que permite obter uma Base Standard para $\mathcal{O} d \mathcal{O}$. As notações usadas nessa seção são as mesmas adotadas na subseção 2.1.1.

\subsection{Invariantes Aritméticos}

O problema central da teoria é a classificação dos ramos, módulo a relação de equivalência abaixo.

Definição 3.1 Se $C_{1}$ e $C_{2}$ são dois ramos dados pelas séries formais $f_{1}$, respectivamente $f_{2}$ de $K[[X, Y]]$, então dizemos que $C_{1}$ é equivalente $a C_{2}$, escrevendo neste caso $C_{1} \sim C_{2}$, se existem uma unidade u e um automorfismo $\Phi$ de $K[[X, Y]]$, tais que

$$
\Phi(f)=u g
$$


É fato bem conhecido que dois ramos são equivalentes se, e somente se, seus anéis locais são isomorfos. $\mathrm{Na}$ verdade, se definirmos a equivalência de curvas algebróides irredutíveis, não necessariamente planas, pelo isomorfismo de seus anéis locais, então quando $K=\mathbb{C}$ e as curvas são analíticas, a definição anterior coincide com a equivalência analítica.

No restante desta seção, estaremos interessados em estudar invariantes aritméticos com respeito à relação de equivalência de curvas, acima definida.

É imediado verificar que a multiplicidade de um ramọ é $11 \mathrm{~m}$ tal invariante aritmético.

Uma vez que, para toda unidade $u$ e todo automorfismo $\Phi$ de $K[[X, Y]]$ temos que.

$$
\operatorname{dim}_{K} \frac{K[[X, Y]]}{\langle f, g\rangle}=\operatorname{dim}_{K} \frac{K[[X, Y]]}{\left\langle u^{-1} \Phi(f), u^{-1} \Phi(g)\right\rangle},
$$

e que $u^{-1} \Phi(g)$.percorre $K[[X, Y]]$ quando $g$ percorre $K[[X, Y]]$, vemos que o semigrupo $\Gamma$ de $C$ é um invariante aritmético da relação de equivalência de ramos.

Como já vimos anteriormente na Definição 2.2, duas curvas $C_{1}$ e $C_{2}$ são equisingulares $\left(C_{1} \equiv C_{2}\right)$ se, e somente se, os semigrupos de $C_{1}$ e $C_{2}$ coincidem. Quando $K=\mathbb{C}$ e no caso de ramos analíticos, a relação de equisingularidade corresponde a equivalência topológica.

Lembre-se que as ordens dos elementos de uma Base Standard Mínima do anel local $\mathcal{O}$ nos dão os elementos dos sistema mínimo de geradores do semigrupo $\Gamma$ associado a uma curva algebróide irredutível $C$, não necessariamente plana. Portanto, dadas duas curvas $C_{1}$ e $C_{2}$ podemos decidir se estas são equisingulares aplicando o Algoritmo 2.1.

$\mathrm{Na}$ verdade, o semigrupo de valores e o conjunto de lacunas especiais de uma curva algebróide irredutível são invariantes aritméticos, como mostra a proposição abaixo.

Proposição 3.1 O semigrupo de valores $\Gamma$, bem como o. conjunto $\Lambda=$ $\{v(\omega)+1 ; \omega \in \mathcal{O} d \mathcal{O}\}$ são invariantes com respeito a relação de equivalência de curvas algebróides irredutiveis.

Dem.: Note que, quando nos restingimos ao caso de ramos a invariância do semigrupo de valores já foi constatada.

Sejam $C_{1}$ e $C_{2}$ em $K\left[\left[X_{1}, \ldots, X_{n}\right]\right]$, duas curvas algebróides irredutíveis equivalentes, ou seja, seus anéis locais $\mathcal{O}_{1}$, respectivamente $\mathcal{O}_{2}$ são isomorfos. 
$\mathrm{O}$ isomorfismo entre os anéis locais se estende de maneira úmica aos corpos de frações $\mathcal{K}_{1}$ de $\mathcal{O}_{1}$, respectivamente $\mathcal{K}_{2}$ de $\mathcal{O}_{2}$.

Sejam $\overline{\mathcal{O}}_{1}=K\left[\left[t_{1}\right]\right]$ e $\overline{\mathcal{O}}_{2}=K\left[\left[t_{2}\right]\right]$ os fechos inteiros de $\mathcal{O}_{1}$ em $\mathcal{K}_{1}$, respectivamente de $\mathcal{O}_{2}$ em $\mathcal{K}_{2}$. A extensão do isomorfismo dos anéis locais aos corpos de frações, permite definir um $K$-isomorfismo

$$
\Phi: \overline{\mathcal{O}}_{2} \longrightarrow \overline{\mathcal{O}}_{1}
$$

Como $\Phi$ preserva ordens, temos

$$
\Phi\left(t_{2}\right)=u t_{1},
$$

onde $u$ é uma unidade de $K\left[\left[t_{1}\right]\right]$.

Segue deste modo, que $\Gamma_{2}=v\left(\mathcal{O}_{2}\right)=v\left(\Phi\left(\mathcal{O}_{2}\right)\right)=v\left(\mathcal{O}_{1}\right)=\Gamma_{1}$, on seja, o semigrupo de valores é um invariante aritmético com respeito a equivalência de curvas. Mais ainda, $\Phi$ preserva Base Standard Mínima de anéis locais, i.e., se $B=\left\{y_{0}, \ldots, y_{g}\right\}$ é uma Base Standard Mínima de $\mathcal{O}_{2}$, então $\Phi(B)$ é uma Base Standard Mínima de $\mathcal{O}_{1}$.

Sejam os homomorfismos $\varphi_{j}$ e $\psi_{j}$ definidos em (2.1), respectivamente em (2.2) para $j=1$, 2. Se $\Omega_{2}=\sum_{i=0}^{g} g_{i} d y_{i} \in \mathcal{O}_{2} d \mathcal{O}_{2} \operatorname{com} g_{i} \in \mathcal{O}_{2}$, então

$$
\psi_{2}\left(\Omega_{2}\right)=\sum_{i=0}^{g} \varphi_{2}\left(g_{i}\right) \varphi_{2}\left(y_{i}\right)^{\prime} \in \overline{\mathcal{O}}_{2} .
$$

Como

$$
\Phi\left(\psi_{2}\left(\Omega_{2}\right)\right)=\sum_{i=0}^{g} \Phi\left(\varphi_{2}\left(g_{i}\right)\right) \Phi\left(\varphi_{2}\left(y_{i}\right)^{\prime}\right)
$$

e $\Phi\left(\varphi_{2}\left(g_{i}\right)\right)=\varphi_{1}\left(h_{i}\right)$ com $h_{i} \in \mathcal{O}_{1}$, temos que

$$
\Phi\left(\psi\left(\Omega_{2}\right)\right)=\sum_{i=0}^{g} \varphi_{1}\left(h_{i}\right) \Phi\left(\varphi_{2}\left(y_{i}\right)^{\prime}\right) .
$$

Agora observe que

$$
\Phi\left(\varphi_{2}\left(y_{i}\right)\right)^{\prime}=\left(u+u^{\prime} t_{1}\right) \Phi\left(\varphi_{2}\left(y_{i}\right)^{\prime}\right)
$$

i.e.,

$$
\Phi\left(\varphi_{2}\left(y_{i}\right)^{\prime}\right)=w_{1} \varphi_{1}\left(z_{i}\right)^{\prime},
$$

onde $w_{1}$ é uma unidade em $K\left[\left[t_{1}\right]\right]$ que não depende de $y_{i}$ e $\Phi(B)=$ $\left\{z_{0}, \ldots, z_{g}\right\}$. 
Desse modo,

$$
\Phi\left(\psi_{2}\left(\Omega_{2}\right)\right)=\left(\sum_{i=0}^{g} \varphi_{1}\left(h_{i}\right) \varphi_{1}\left(z_{i}\right)^{\prime}\right) w_{1}=\psi_{1}\left(\Omega_{1}\right) w_{1}
$$

$\operatorname{com} \Omega_{1} \in \mathcal{O}_{1} d \mathcal{O}_{1}$

Assim, temos

$$
v\left(\Omega_{2}\right)=v\left(\psi_{2}\left(\Omega_{2}\right)\right)=v\left(\Phi\left(\psi_{2}\left(\Omega_{2}\right)\right)\right)=v\left(\psi_{1}\left(\Omega_{1}\right)\right)=v\left(\Omega_{1}\right),
$$

ou seja, $\Lambda_{2} \subseteq \Lambda_{1}$.

De modo análogo, mostra-se que $\Lambda_{1} \subseteq \Lambda_{2}$ e portanto, $\Lambda_{1}=\Lambda_{2}$.

Observação 3.1 Note que pela proposição anterior temos que $\Phi\left(\varphi_{2}\left(\mathcal{O}_{2}\right)\right)=\varphi_{1}\left(\mathcal{O}_{1}\right)$, no entanto, $\Phi\left(\psi_{2}\left(\mathcal{O}_{2} d \mathcal{O}_{2}\right)\right) \neq \psi_{1}\left(\mathcal{O}_{1} d \mathcal{O}_{1}\right)$. Na verdade, temos

$$
\Phi\left(\psi_{2}\left(\mathcal{O}_{2} d \mathcal{O}_{2}\right)\right)=\psi_{1}\left(\mathcal{O}_{1} d \mathcal{O}_{1}\right) w_{1}
$$

com $w_{1}$ como descrito na demonstração da proposição acima.

De fato, temos que

$$
\Phi\left(\psi_{2}\left(\Omega_{2}\right)\right)=\psi_{1}\left(\Omega_{1}\right) w_{1} \text { e } \Phi^{-1}\left(\psi_{1}\left(\Omega_{1}\right)\right)=\psi_{2}\left(\Omega_{2}\right) w_{2},
$$

com $w_{1} \in K\left[\left[t_{1}\right]\right]$ e $w_{2} \in K\left[\left[t_{2}\right]\right]$ unidades que não dependem das diferenciais $\Omega_{1}$ e $\Omega_{2}$, mas sim do $K$-isomorfismo $\Phi$. Segue assim, que $\Phi\left(w_{2}\right) w_{1}=1$.

Como

$$
\Phi\left(\psi_{2}\left(\mathcal{O}_{2} d \mathcal{O}_{2}\right)\right) \subseteq \psi_{1}\left(\mathcal{O}_{1} d \mathcal{O}_{1}\right) w_{1} \subseteq \Phi\left(\psi_{2}\left(\mathcal{O}_{2} d \mathcal{O}_{2}\right)\right) \Phi\left(w_{2}\right) w_{1}
$$

temos que $\Phi\left(\psi_{2}\left(\mathcal{O}_{2} d \mathcal{O}_{2}\right)\right)=\psi_{1}\left(\mathcal{O}_{1} d \mathcal{O}_{1}\right) w_{1}$.

Lembremos que $\Gamma \subseteq \Lambda$, mas $\Lambda$ não determina, em geral, o semigrupo $\Gamma$, veja- Exemplo 2.5 .

Resaltamos que os algoritmos apresentados para obter uma Base Standard Mínima para o anel local $\mathcal{O}$ e para o módulo de diferenciais $\mathcal{O} d \mathcal{O}$, conseqüentemente para obter $\Gamma$ e $\Lambda$, permitem que reconheçamos quando duas curvas algebróides irredutíveis são equisingulares e equidiferenciáveis. Assim, o estudo da equivalência de curvas pode ser reduzido ao conjunto das curvas que possuem os mesmos conjuntos $\Gamma$ e $\Lambda$.

No caso de curvas algebróides irredutíveis que são interseção completa, mostramos no Corolário 2.1 que o número $\tau$ de Tjurina pode ser obtido por $\Lambda$ e $\Gamma, \operatorname{assim} \tau$ é também um invariante aritmético com respeito à equivalência de curvas algebróides irredutíveis. 


\subsection{Classificação de Curvas}

Como já mencionamos anteriormente, um dos problemas centrais da teoria, até o momento não resolvido, é decidir quando dois ramos $f_{1}$ e $f_{2}$ são equivalentes. Obviamente esses ramos devem possuir o mesmo semigrupo de valores, i.e., devem pertencer a mesma classe de equisingularidade $\Gamma$.

$O$ primeiro passo é encontrar formas normais para os ramos de $11 \mathrm{ma}$ mesma classe de equisingularidade para posteriormente obter critérios para decidir quando dois ramos na forma normal são equivalentes.

Dado um ramo, i.e., uma série irredutível $f \in K[[X, Y]]$ e $\mu$ o coudutor do seı semigrupo de valores $\Gamma$, pelo Teorema da Fuução Implícita de Tougeron (Veja Prop. 2.3 de $[R u]$ ), temos que $f$ é equivalente ao seu truncameuto $g$ até a ordem $2 \mu$, ou seja, $f-g \in \mathcal{M}_{X, Y}^{2 \mu+1}$ oude $\mathcal{M}_{X, Y}$ é o ideal maximal de $K[[X, Y]]$.

Após mudanças de coordenadas, facilmente determinadas, que correspondem ao Teorema de Preparação de Weierstrass (Veja Prop. 3.3 de [Ru]), podemos considerar

$$
g=Y^{v_{0}}+a_{1}(X) Y^{v_{0}-1}+\cdots+a_{v_{0}}(x),
$$

com $a_{i}(X)$ polinômios tais que $\operatorname{ord}_{X} a_{i}(X)>i$ para $1 \leq i \leq v_{0}, \operatorname{deg}_{X} a_{i}(X)+$ $v_{0}-i \leq 2 \mu$ e $v_{0} \not v_{1}$, onde $v_{0}=\operatorname{mult}(g)$ e $v_{1}=I(g, Y)$.

Pelo Teorema de Newton-Puiseux (Veja Prop. 4.4 de [Ru]), existe uma parametrização de $g$ da forma

$$
\left\{\begin{array}{l}
x=t^{v_{0}} \\
y=t^{v_{1}}+\sum_{i>v_{1}} b_{i} t^{i} .
\end{array}\right.
$$

$O$ método de Newton-Puiseux para determinar estas parametrizações, apesar de construtivo, envolve, eventualmente, um número infinito de operações. Veremos mais adiante que um uúmero finito de termos na série $y$, mais precisamente o seu truncamento até a ordem $\mu$, determina uma curva equivalente a $g$ e portanto, uma forma normal para a curva original $f$.

$\dot{E}$ fato conhecido que os expoentes característicos $\beta_{0}, \ldots, \beta_{g}$, como definidos na Subseção 2.1.1, determinam e são determinados pelo semigrupo de valores $\Gamma$. Obtemos assim, um aberto de um espaço afim

$$
K^{\mu-1-v_{1}} \backslash H
$$

com $H=\cup_{i=2}^{g}\left\{b_{\beta_{i}}=0\right\}$, que contém pelo menos um representante de cada classe de equivalência de curvas na classe de equisingularidade dada por $\Gamma$. 
É portanto necessário, saber reconhecer quando duas parametrizações de Puiseux, representam curvas equivalentes, isto equivale a estıudar critérios de isomorfismos para subáneis de $K[[t]]$, do tipo $K\left[\left[t^{v_{0}}, t^{v_{1}}+\sum_{i>v_{1}} a_{i} t^{i}\right]\right]$, que denominamos áneis de Puiseux.

A proposição abaixo, estabelece critérios de isomorfismos para anéis dẹ Puiseux quaisquer.

Proposição $3.2 \operatorname{Sejam} \mathcal{A}_{1}=K\left[\left[t_{1}^{v_{0,1}}, p_{1}\left(t_{1}\right), \ldots, p_{r}\left(t_{1}\right)\right]\right] \subseteq K\left[\left[t_{1}\right]\right]$ e $\mathcal{A}_{2}=$ $K\left[\left[t_{2}^{v_{0,2}}, q_{1}\left(t_{2}\right), \ldots, q_{r}\left(t_{2}\right)\right]\right] \subseteq K\left[\left[t_{2}\right]\right]$ anéis de Puiseux, onde $p_{i}\left(t_{j}\right)=t_{j}^{v_{i, j}}+\cdots$, $\operatorname{com} v_{0, j}<v_{i, j}$ e $v_{k, j} \notin\left\langle v_{0, j}, \ldots, \widehat{v_{k, j}}, \ldots, v_{r, j}\right\rangle$ para $j=1,2, i=1, \ldots, r$ e $k=0, \ldots, r$.

As $K$-álgebras $\mathcal{A}_{1}$ e $\mathcal{A}_{2}$ são isomorfas se, e somente se,

1. $v_{k, 1}=v_{k, 2}=: v_{k}$ para todo $k=0, \ldots, r$.

2. Existem $P_{k} \in \mathcal{A}_{1}$ para $k=0, \ldots, r, c \in K^{*}$, com $v\left(P_{k}\right)>v_{k}$, с. um $K$-isomorfismo

$$
\Phi: K\left[\left[t_{2}\right]\right] \longrightarrow K\left[\left[t_{1}\right]\right]
$$

tais que

$$
\Phi\left(t_{2}\right)=c t_{1}\left(1+\frac{P_{0}}{t_{1}^{v_{0}}}\right)^{\frac{1}{v_{0}}}
$$

$e$

$$
\Phi\left(q_{i}\right)=c^{v_{i}} p_{i}+P_{i}
$$

para todo $i=1, \ldots, r$.

Dem.: Inicialmente note que as condições 1) e 2) indicam que $\Phi\left(\mathcal{A}_{2}\right) \subseteq \mathcal{A}_{1}$ e que $\Phi^{-1}\left(\mathcal{A}_{1}\right) \subseteq \mathcal{A}_{2}$. Portanto, o $K$-isomorfismo $\Phi$ se restringe a um $K$ isomorfismo entre $\mathcal{A}_{2}$ e $\mathcal{A}_{1}$.

Reciprocamente, como na Proposição 3.1, um $K$-isomorfismo entre os anéis de Puiseux $\mathcal{A}_{1}$ e $\mathcal{A}_{2}$, se estende a um $K$-isomorfismo $\Phi$ entre seus fechos integrais, que pela Observação 2.1 são $K\left[\left[t_{1}\right]\right]$, respectivamente $K\left[\left[t_{2}\right]\right]$. Como $\Phi$ preserva ordens, a condição 1) segue.

Sabemos que $\Phi$ é tal que $\Phi\left(t_{2}\right)=u t_{1}$, onde $u$ é uma unidade de $K\left[\left[t_{1}\right]\right]$.

Como $\Phi\left(t_{2}^{v_{0}}\right)=\Phi\left(t_{2}\right)^{v_{0}}=u^{v_{0}} t_{1}^{v_{0}} \in \mathcal{A}_{1}$, podemos escrever $t_{1}^{v_{0}} u^{v_{0}}=c_{1}\left(t_{1}^{v_{0}}+\right.$ $\left.P_{0}\right) \operatorname{com} P_{0} \in \mathcal{A}_{1}$ e $v\left(P_{0}\right)>v_{0}$. Temos assim que $u^{v_{0}}=c_{1}\left(1+\frac{P_{0}}{t_{1}^{0}}\right)$, i.e.,

$$
u=c\left(1+\frac{P_{0}}{t_{1}^{v_{0}}}\right)^{\frac{1}{v_{0}}},
$$


onde $c=c_{1}^{\frac{1}{v_{0}}}$ é unicamente determinado.

De modo análogo, como $v_{k} \notin\left\langle v_{0}, \ldots, \widehat{v}_{k}, \ldots, v_{r}\right\rangle$, para todo $k=0, \ldots, r$, temos que

$$
\Phi\left(q_{i}\left(t_{2}\right)\right)=c^{v_{i}} p_{i}\left(t_{1}\right)+P_{i}
$$

com $P_{i} \in \mathcal{A}_{1}$ univocamente determinado e $v\left(P_{i}\right)>v_{i}$ para todo $i=1, \ldots, r$.

Dado um anel de Puiseux $\mathcal{A}_{1}=K\left[\left[t_{1}^{v_{0}}, p_{1}\left(t_{1}\right), \ldots, p_{r}\left(t_{1}\right)\right]\right]$, escolhidos $P_{k} \in$ $\mathcal{A}_{1}$ para $k=0, \ldots, r$ e $c \in K^{*}$ satisfazendo as condições da proposição anterior, ou seja, $p_{i}\left(t_{1}\right)=t_{1}^{v_{i}}+\cdots$ com $v_{0}<v_{i}$ e $v_{k} \notin\left\langle v_{0}, \ldots, \widehat{v}_{k}, \ldots, v_{r}\right\rangle$ para $i=1, \ldots, r$ e $k=0, \ldots, r$. Temos que $\mathcal{A}_{1}$ é $K$-isomorfo ao anel de Puisenx $\mathcal{A}_{2}=K\left[\left[t_{2}^{v_{0}}, q_{1}\left(t_{2}\right), \ldots, q_{r}\left(t_{2}\right)\right]\right]$, oude

$$
t_{2}=c t_{1}\left(1+\frac{P_{0}}{t_{1}^{v_{0}}}\right)^{\frac{1}{v_{0}}}
$$

e

$$
q_{i}=c^{\boldsymbol{v}_{i}} p_{i}+P_{i}
$$

para todo $i=1, \ldots, r$.

Neste caso, dizemos que $\mathcal{A}_{2}$ é obtido de $\mathcal{A}_{1}$ por mudanças de coordenadas.

Retornemos ao estudo da equivalência de ramos, on seja, aos anéis de Puiseux dados por $\mathcal{A}=K\left[\left[t^{v_{0}}, y\right]\right]$, onde $y=t^{v_{1}}+\sum_{i>v_{1}} b_{i} t^{i} \operatorname{com} v_{0}<v_{1} \mathrm{e}$ $v_{0} \backslash v_{1}$.

\section{Exemplo 3.1 (HOMOTETIAS)}

Mudanças de coordenadas do tipo

$$
\begin{gathered}
t_{2}=c t_{1} \\
y_{2}=c^{v_{1}} y_{1},
\end{gathered}
$$

com $c \in K^{*}$, são chamadas de homotetias.

Se $y_{1}=t_{1}^{v_{1}}+\sum_{i>v_{1}} b_{i} t^{i}$, então

$$
y_{2}=c^{v_{1}}\left(t_{1}^{v_{1}}+\sum_{i>v_{1}} b_{i} t_{1}^{i}\right)=\left(c t_{1}\right)^{v_{1}}+\sum_{i>v_{1}} b_{i} c^{v_{1}-i}\left(c t_{1}\right)^{i}=t_{2}^{v_{1}}+\sum_{i>v_{1}} b_{i} c^{v_{1}-i} t_{2}^{i}
$$

Veja que esse tipo de mudança não permité eliminar nenhum termo em $y_{2}$. No entanto, podemos tornar o coeficiente de qualquer termo presente, igual a um, escolhendo $c=b_{i}^{\frac{1}{i-v_{1}}}$, onde $b_{i}$ é o coeficiente do termo em questão. 
Exemplo 3.2 Consideremos as mudanças de coordenadas do tipo

$$
t_{2}=\Phi\left(t_{1}\right)=t_{1}\left(1+\frac{p}{t_{1}^{v_{0}}}\right)^{\frac{1}{v_{0}}} \text { e } y_{2}=y_{1}+q
$$

Queremos determinar $y_{2}\left(t_{2}\right)=y_{1}\left(\Phi^{-1}\left(t_{2}\right)\right)+q\left(\Phi^{-1}\left(t_{2}\right)\right)$. Como $\Phi^{-1}\left(t_{2}\right)=$ $t_{2}+h\left(t_{2}\right)$, temos que $y_{1}\left(\Phi^{-1}\left(t_{2}\right)\right)=y_{1}\left(t_{2}\right)+H\left(\Phi\left(t_{2}\right)\right)$, onde

$$
H\left(\Phi\left(t_{2}\right)\right)=\sum_{i \geq v_{1}} b_{i} t_{1}^{i}\left(\sum_{j=1}^{\infty}\left(\begin{array}{c}
\frac{i}{v_{0}} \\
j
\end{array}\right)\left(\frac{p}{t_{1}^{v_{0}}}\right)^{j}\right) .
$$

Desse modo, teremos

$$
\begin{aligned}
& x_{2}=t_{2}^{v_{0}} \\
& y_{2}=t_{2}^{v_{1}}+\sum_{i>v_{1}} b_{i} t_{2}^{i}+q-p \frac{d y_{1}}{d x_{1}}-\sum_{i \geq v_{1}} b_{i} t_{1}^{i}\left(\sum_{j=2}^{\infty}\left(\begin{array}{c}
\frac{i}{v_{0}} \\
j
\end{array}\right)\left(\frac{p}{t_{1}^{v_{0}}}\right)^{j}\right) .
\end{aligned}
$$

Vemos que se

$$
\frac{q d x_{1}-p d y_{1}}{d x_{1}}-\sum_{i \geq v_{1}} b_{i} t_{1}^{i}\left(\sum_{j=2}^{\infty}\left(\begin{array}{c}
\frac{1}{v_{0}} \\
j
\end{array}\right)\left(\frac{p}{t_{1}^{v_{0}}}\right)^{j}\right)=d t_{1}^{l}+\begin{gathered}
\text { termos de } \\
\text { ordem } \\
\text { superior }
\end{gathered}
$$

então podemos substituir $p$ e $q$ por $\alpha p$ e $\alpha q$ de modo a obtermos $d=-b_{l}$. Assim, a mudança acima, elimina o termo de ordem l em $y_{2}\left(t_{2}\right)$, sem alterar os temos de ordem inferior.

Em particular, se $l \in \Gamma$, então podemos tomar $p=0$ e $q \in \mathcal{O}$, com $v(q)=l$. Se $l \in \Gamma+v_{1}-v_{0}$, então podemos tomar $q=0$ e $p \in \mathcal{O}$, com $v(p)=l-v_{1}+v_{0}$. Desta forma, podemos sempre eliminar os t.erm.os na parametrização de Puiseux de um ramo cuja ordem estão em $\Gamma \cup\left(\Gamma+v_{1}-v_{0}\right)$, sem que alteremos os termos 'de ordem inferior.

Toda mudança de coordenadas de um anel de Puiseux é composição de uma mudança do Exemplo 3.1 e uma mudança do Exemplo 3.2.

Deste ponto em diante, para facilitar nossos cálculos, estaremos considerando as parametrizações sem os termos que podemos eliminar como descrito acima.

Definição 3.2 O conjunto que parametriza todas as curvas com semigrupo $\Gamma$ fuxo, módulo a relação de equivalência de curvas, será chamado de classe de equisingularidade e denotado por $\mathbf{M}_{\Gamma}$. 
Note que pelo Exemplo 3.2, para efeito de classificação de ramos, basta considerarmos parametrizações de Puisenx truncadas no condutor $\mu$ do semigrupo $\Gamma$ do ramo, isto justifica o fato mencionado no início desta seção.

Observação 3.2 Vamos caracterizar as lacunas do semigrupo $\Gamma$ maiores que $v(y)=v_{1}$, uma vez que dentre elas estão as potências na representação paramétrica que estaremos considerando.

Seja $\Gamma^{\prime}=\left\langle v_{0}, v_{1}, \ldots, v_{g}\right\rangle$ o semigrupo de valores de uma curva invedutinel plana e $\left(a_{i}\right)_{i=0, \ldots, v_{0}-1}$ a seqüêencia de Apéry de $\Gamma$ com relação à $v_{0}$, ou seja,

$$
a_{0}=0 \quad e \quad a_{i}=\min \left\{\Gamma \backslash \cup_{j=0}^{i-1}\left\{a_{j}+v_{0} \mathbb{N}\right\}\right\},
$$

para $i=1, \ldots, v_{0}-1$. A saber, temos que

$$
\left\{a_{0}, a_{1}, \ldots, a_{v_{0}-1}\right\}=\left\{\sum_{i=1}^{g} s_{i} v_{i} ; 0 \leq s_{i} \leq n_{i}\right\},
$$

onde $n_{i}=\frac{M D C\left(v_{0}, \ldots, v_{i-1}\right)}{M D C\left(v_{0}, \ldots, v_{i}\right)}$.

Um número natural l é uma lacuna de $\Gamma$ maior que $a_{1}=v_{1}$ se, e somente. se, $l=a_{i}-j v_{0}$ para algum $i=2, \ldots, v_{0}-1$ e algum $j=1, \ldots,\left[\frac{a_{i}-v_{1}}{v_{0}}\right]$.

De fato, os inteiros da forma $a_{i}-j v_{0}$ com $j>0$ são obviamente lacunas de $\Gamma$ e a condição $a_{i}-j v_{0}>v_{1}$ é equivalente $a .1 \leq j \leq\left[\frac{a_{i}-v_{1}}{v_{0}}\right]$.

Seja

$$
C:\left\{\begin{array}{l}
x=t^{v_{0}} \\
y=t^{v_{1}}+\sum_{i>v_{1}} b_{i} t^{i},
\end{array}\right.
$$

$\operatorname{com} i \notin \Gamma \cup\left(\Gamma+v_{1}-v_{0}\right)$.

Ao aplicarmos o algoritmo para obter uma Base Standard Mínima para $\mathcal{O} d \mathcal{O}$, partindo de uma parametrização de $C$, o $S$-processo de menor altura é $x d y-\frac{v_{1}}{v_{0}} y d x=\sum_{i>v_{1}}\left(i-v_{1}\right) b_{i} t^{i+v_{0}-1}$.

Se $b_{i}=0$ para todo $i>v_{1}$, então

$$
C:\left\{\begin{array}{l}
x=t^{v_{0}} \\
y=t^{v_{1}}
\end{array}\right.
$$

$\operatorname{com} \operatorname{MDC}\left(v_{0}, v_{1}\right)=1$, pois estamos assumindo que a parametrização de $C \dot{e}$ primitiva. Além disso, o algortimo finaliza, nos levando a concluir que $\Lambda=\Gamma$ e $\tau=\mu$. 
Se existe um $b_{i} \neq 0$; então pondo $\lambda=\min \left\{i ; b_{i} \neq 0\right\}$, temos que $\Omega=$ $x d y-\frac{v_{1}}{v_{0}} y d x$ é tal que $v(\Omega)+1=\lambda+v_{0}$. A diferencial $\Omega$ é não exata, pois $\lambda+v_{0} \notin \Gamma$. De fato, $\lambda=k v_{1}-j v_{0}$ com $j \geq 2$ ou $\lambda=\beta_{2}=v_{2}+v_{1}-\frac{v_{1} v_{0}}{e_{1}}$, $\operatorname{com} e_{1}=\operatorname{MDC}\left(v_{0}, v_{1}\right)$ e $\frac{v_{1}}{e_{1}}>2$. Em ambos os casos, tem-se que $\lambda+v_{0} \notin \Gamma$. $\mathrm{Na}$ verdade $\lambda+v_{0}$ é a menor ordem que uma DNE pode assumir. Assim, $\lambda+v_{0}=\min \{l ; l \in \Lambda \backslash \Gamma\}$, e portanto, $\lambda$ é um invariante com relação a equivalência de curvas.

Pelo que observamos acima, uma curva tem $\tau$ máximo se, e somente se, ela é equivalente a curva

$$
\left\{\begin{array}{l}
\tau=t^{v_{0}} \\
y=t^{v_{1}}
\end{array}\right.
$$

Estes fatos foram mostrados por Zariski em [Z1].

A lacuna $\lambda$, definida acima, será chamada de invariante de Zariski.

Observação 3.3 1. O limitante superior para $\tau$ é.o condutor $\mu$ do semigrupo de valores $\Gamma$ associado à $C$, um limitante inferior para $\tau$ é $\frac{\mu}{2}-\left[\frac{v_{1}}{v_{0}}\right]+v_{1}+v_{0}-3$. De fato, sabemos que $\tau=\mu-\sharp(\Lambda \backslash \Gamma)$, onde $\Lambda=\{v(\omega)+1 ; \omega \in \mathcal{O} d \mathcal{O}\}$. Vamos supor que $\Lambda \backslash \Gamma \neq\{\}$, pois caso contrário $\tau=\mu$, como vimos anteriormente. Seja $v(\omega)+1 \in \Lambda \backslash \Gamma$, como o menor valor que $v(\omega)+1$ pode assumir é $\lambda+v_{0}>v_{1}+v_{0}$, temos que $v(\omega)+1 \in\left\{l \notin \Gamma ; l>v_{1}+v_{0}\right\}$.

Os elementos de $\Gamma$ menores ou iguais a $v_{1}+v_{0}$ são:

$$
\left\{0, v_{0}, 2 v_{0}, \ldots,\left[\frac{v_{1}}{v_{0}}\right] v_{0}, v_{1},\left(\left[\frac{v_{1}}{v_{0}}\right]+1\right) v_{0}, v_{1}+v_{0}\right\}
$$

ou seja, existem. $v_{1}+v_{0}-\left[\frac{v_{1}}{v_{0}}\right]-3$ lacunas menores que $v_{1}+v_{0}$. Assim,

$$
\frac{\mu}{2}+v_{1}+v_{0}=\left[\frac{v_{1}}{v_{0}}\right]-3 \leq \tau \leq \mu .
$$

Note ainda, que uma condição necessária para $\tau<\mu$ é que $v_{0}>2$.

2. Pela Observação 3.2, temos que uma Base Standard Mínima de $\mathcal{O} d \mathcal{O}$ possui no máximo v $v_{0}-2$ DNEM's. De fato, se $\omega_{1}$ e $\omega_{2}$ são DNEM's, $\operatorname{com} v\left(\omega_{1}\right)+1 \equiv a_{i} \bmod v_{0} e v\left(\omega_{2}\right)+1 \equiv a_{i} \bmod v_{0}$, então $v\left(\omega_{1}\right)-v\left(\omega_{2}\right)=$ $k v_{0}$. Se $k \geq 0$, então $v\left(\omega_{1}\right)=v\left(x^{k} \omega_{2}\right)$, caso $k<0$ temos $v\left(\omega_{2}\right)=$ $v\left(x^{-k} \omega_{1}\right)$, assim uma das DNE não seria minimal. Como a menor 
ordem que uma DNEM pode assumir é $\lambda+v_{0}-1>v_{1}+v_{0}-1>a_{1}=v_{1}$, devemos ter no máximo $v_{0}-2$ DNEM's. Note ainda que a DNE de. menor ordem em um dado passo do algoritmo é minimal. De fato, tal DNE não se reduz módulo as diferenciais minimais obtidas nos passos anteriores e, nos próximos passos, todas as $D N E$ terão ordem superior a esta. Desse modo, temos

$$
\begin{gathered}
\hline \begin{array}{c}
\text { número de passos } \\
\text { do algoritmo }
\end{array} \\
\text { número de DNEM's } \leq v_{0}-2 \\
\end{gathered}
$$

Seja $\Gamma=\left\langle v_{0}, v_{1}, \ldots, v_{g}\right\rangle$ o semigrupo associado a $u$ m ramo e $\sigma \in \mathbb{N}$ com $\sigma>\left[\frac{a_{v_{0}-1}-v_{1}}{v_{0}}\right] \geq\left[\frac{a_{2}-v_{1}}{v_{0}}\right]$. Como cada lacuma $l>v_{1}$ é expressa unicamente na forma $l=a_{i}-j v_{0}=\sum_{k=1}^{g} s_{k} v_{k}-s_{0} v_{0}, 0 \leq s_{k}<n_{k}, 0<s_{0} \leq\left[\frac{a_{i}-v_{1}}{v_{0}}\right]<\sigma$, onde $n_{k}=\frac{\operatorname{MDC}\left(v_{0}, \ldots, v_{k-1}\right)}{\operatorname{MDC}\left(v_{0}, \ldots, v_{k}\right)}$ e as lacunas de $\Gamma$ que estão em $\Lambda$ possuem essa propriedade, podemos representar os elementos de $\Lambda$ em $\mathbb{N}^{g+1}$, através da aplicação

$$
\varrho: \begin{array}{ccc}
\Lambda & \mathbb{N}^{g+1} \\
\sum_{k=1}^{g} s_{k} v_{k}-s_{0} v_{0} & \mapsto & \left(\sigma-s_{0}, s_{1}, \ldots, s_{g}\right)
\end{array}
$$

Utilizaremos constantemente a representação de um inteiro sob a forma $\sum_{k=1}^{g} s_{k} v_{k}-s_{0} v_{0}$, com $0 \leq s_{k}<n_{k}$ e $s_{0} \in \mathbb{Z}$ onde $n_{i}=\frac{\operatorname{MDC}\left(v_{0}, \ldots, v_{i-1}\right)}{\operatorname{MDC}\left(v_{0}, \ldots, v_{i}\right)}$. Tal representação será chamada de escrita única ou escrita privilegiada.

Seja $\omega \in \mathcal{O} d \mathcal{O}$ uma DNEM e considere $v(\omega)+1=\sum_{k=1}^{g} s_{k} v_{k}-s_{0} v_{0}$ na escrita única. Se $F=\left\{f_{0}, \ldots, f_{g}\right\}$ é uma Base Standard Mínima para o anel local $\mathcal{O}$ e $\alpha=\left(\alpha_{0}, \ldots, \alpha_{g}\right) \in \mathbb{N}^{g+1}$, tal que $s_{i}+\alpha_{i}<n_{i}$ e $s_{0}-\alpha_{0}>0$, então $v\left(F^{\alpha} \omega\right)$ é ainda uma lacuna que está em $\Lambda \backslash \Gamma$ e portanto uma lacuna especial (cf. Definição 2.5). Veja que se $\Lambda \neq \Gamma$, então $\max \{\Lambda \backslash \Gamma\}=\mu-1$.

Definição 3.3 O diagrama em $\mathbb{N}^{g+1}$ constituido dos pontos correspondentes as lacunas $v(\omega)+1=\sum_{i=1}^{g} s_{i} v_{i}-s_{0} \eta_{0}, 0 \leq s_{i}<n_{i}, 0<s_{0}<\sigma$ onde. $\omega$ é uma DNE, por meio da aplicação

$$
\sum_{i=1}^{g} s_{i} v_{i}-s_{0} v_{0} \stackrel{\varrho}{\mapsto}\left(\sigma-s_{0}, s_{1}, \ldots, s_{g}\right)
$$

é chamado de diagrama de lacunas especiais de $C$. 
Exemplo 3.3 Seja

$$
C:\left\{\begin{array}{l}
x=t^{8} \\
y=t^{12}+t^{13}
\end{array}\right.
$$

Pelo item 3 do Exemplo 2.4, sabem.os que $\Gamma=\left\langle v_{0}, v_{1}, v_{2}\right\rangle=\langle 8,12,25\rangle$,

$$
\Lambda \backslash \Gamma=\{21,29,34,38,42,46,47,51,54,55,59,63,67,71,79\}
$$

e as lacunas correspondente às ordens de DNEM são $\{21,34,38,47,51\}$.

Temos

$$
\begin{aligned}
& 21=1 v_{2}+1 v_{1}-2 v_{0} \\
& 34=2 v_{2}+0 v_{1}-2 v_{0} \\
& 38=2 v_{2}+1 v_{1}-3 v_{0} \\
& 47=3 v_{2}+1 v_{1}-5 v_{0} \\
& 51=3 v_{2}+0 v_{1}-3 v_{0}
\end{aligned}
$$

Tomando $\sigma=10>\frac{a_{v_{0}-1}-v_{1}}{v_{0}}=\frac{75}{8}$, obtemos o seguinte diagrama de lacunas especiais para $C$ :

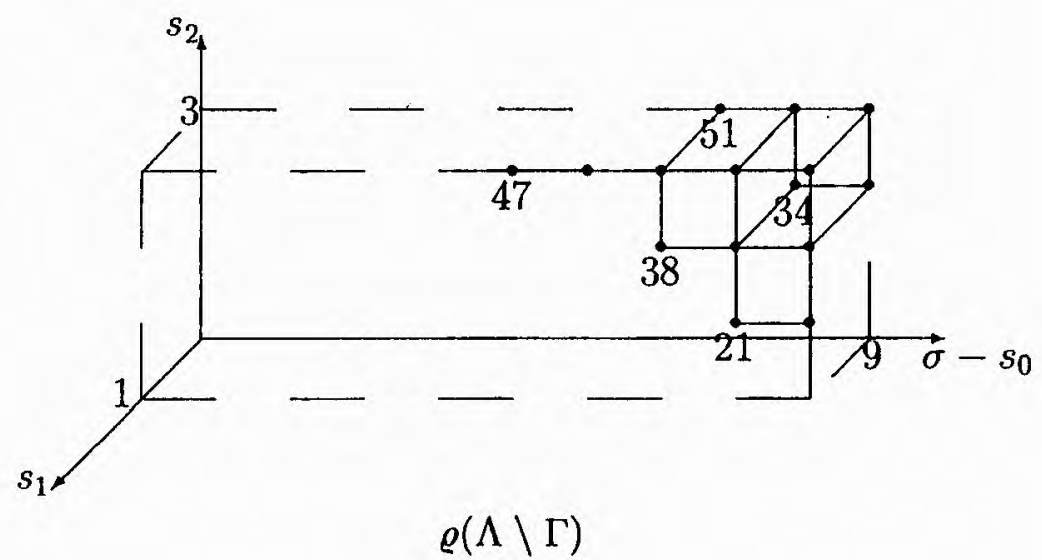

Por simplicidade, representaremos apenas a região que contém os pontos do diagrama. Veja que basta fornecer a indicação de um ponto para que possamos nos orientar e determinar os demais.

Na figura abaixo, apresentamos apenas a região que contém os pontos de $\varrho(\Lambda \backslash \Gamma)$, onde o ponto frontal superior direito corresponde $\grave{a} \mu-1$. 


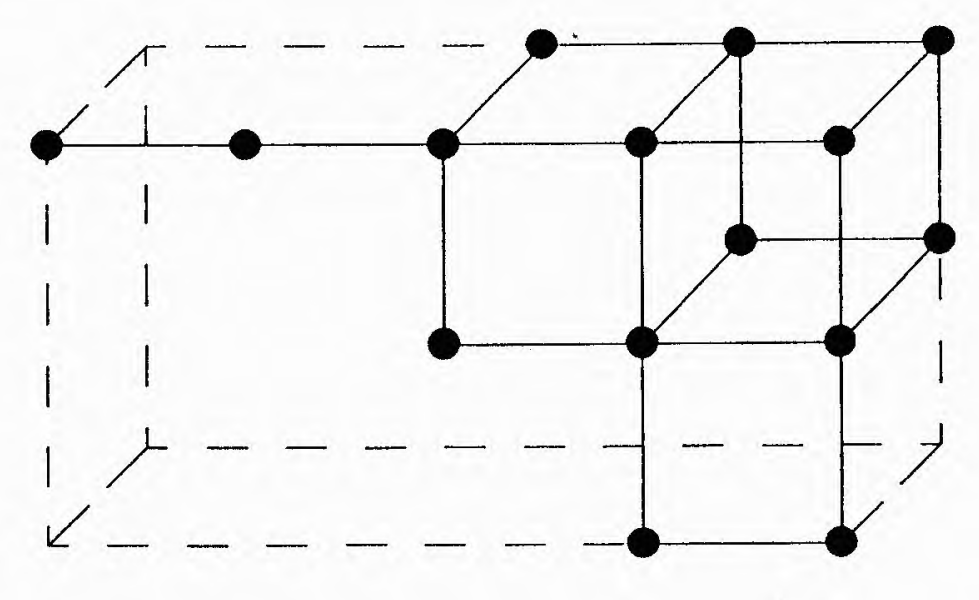

Uma vez que $\Lambda \backslash \Gamma$ é um invariante com respeito à relação de equivalência de curvas algebróides irredutíveis planas, temos que o diagrama de lacunas especiais é também um invariante, uma vez que este é obtido de $\Lambda \backslash \Gamma$.

Observação 3.4 O algoritmo para obter uma Base Standard Minima para $\mathcal{O} d \mathcal{O}$ apresentado no Capítulo 2, é eficaz no sentido de que pode ser aplicado para representações paramétricas com qualquer grau de generalidade, i.e., podemos aplicar o algortimo para uma curva genérica, ou seja, para uma escolha arbitrária dos coeficientes e para uma curva específica. Isto permite, entre outras coisas, o estudo completo do invariante $\Lambda \backslash \Gamma$ para uma dada classe de equisingularidade. Como conseqüência, podemos determinar todos os possiveis números de Tjurina na classe de equisingularidade, bem como descrever todas as classes de equidiferenciabilidade.

A determinação do número de Tjurina mínimo numa classe de equisingularidade e algumas variantes foi abordado em vários trabalhos (cf. [D2], [BGM], [Pe1], [Pe2] e [Z2] ).

R. Peraire, em [Pe1], apresenta um algoritmo para obter o número de Tjurina minimo para uma dada classe de equisingularidade. No entanto, tal algoritmo contrariamente ao nosso, não se aplica a uma curva específica, por se basear apenas na aritmética do semigrupo em questão. 
Em [Pe2], a mesma autora determina o número de Tjurina mínimo para curvas numa classe de equisingularidade de gênero 1 com invariante $\lambda$ de Zariski fixo.

Por outro lado, C. Delorme, em [D2] apresenta um algoritmo para determinar as lacunas especiais para curvas numa classe de equisingularidade de gênero 1, que não se estende para gênero superiores e é menos sistemático do que o nosso.

Na seqüência, apresentamos $11 \mathrm{~m}$ exemplo, onde obtemos todos os possíveis conjuntos $\Lambda$ para uma classe de equisingularidade $M_{\Gamma}$ fixa, ou seja, determinamos todos os estratos $M_{\Gamma, \Lambda}$ do espaço $M_{\Gamma}$ correspondentes às classes de equidiferenciabilidade definidas por $\Lambda$.

Note que, tal análise não poderia ser realizada com as técuicas até cntão disponíveis.

Exemplo 3.4 Seja $\Gamma=\langle 6,9,19\rangle$. A seqüência característica das curvas algebróides irredutíveis planas com semigrupo associado $\Gamma$ é $\beta_{0}=v_{0}=6, \beta_{1}=$ $\dot{v}_{1}=9$ e $\beta_{2}=10$.

Uma vez que as lacunas de $\Gamma$ maiores que $v_{1}=9$ são

$$
\{10,11,13,14,16,17,20,22,23,26,29,32,35,41\},
$$

temos que $\lambda=\beta_{2}=10$.

Como já observamos, podemos considerar através de uma mudança de coordenadas, que o coeficiente do termo de expoente $\lambda$ seja igual a 1 (cf. Exemplo 3.1). Além disso, como $22=v_{2}+v_{1}-v_{0}$ e $41=2 v_{2}+v_{1}-v_{0}$ temos que as curvas determinadas por $\mathbf{M}_{\mathbf{\Gamma}}$ são dadas por:

$$
C_{a}:\left\{\begin{array}{r}
x=t^{6} \\
y=t^{9}+t^{10}+a_{1} t^{11}+a_{2} t^{13}+a_{3} t^{14}+a_{4} t^{16}+a_{5} t^{17}+a_{6} t^{20}+ \\
\\
+a_{7} t^{23}+a_{8} t^{26}+a_{9} t^{29}+a_{10} t^{32}+a_{11} t^{35}
\end{array}\right.
$$

onde $a=\left(a_{1}, \ldots, a_{11}\right) \in K^{11}$. Aplicando a Proposição 2.1, obtemos uma Base Standard Mínima para o anel local $\mathcal{O}$ de $C_{a}$ dada por $F=\{x, y, z\}$, onde

$$
\begin{aligned}
z= & y^{2}-x^{3}=2 t^{19}+\left(1+2 a_{1}\right) t^{20}+2 a_{2} t^{21}+\left(a_{1}^{2}+2 a_{2}\right) t^{22}+ \\
& +\left(2 a_{2}+2 a_{3}\right) t^{23}+\left(2 a_{1} a_{2}+2 a_{3}\right) t^{24}+\left(2 a_{1} a_{3}+2 a_{4}\right) t^{25}+ \\
& +\left(a_{2}^{2}+2 a_{4}+2 a_{5}\right) t^{26}+\left(2 a_{2} a_{3}+2 a_{1} a_{4}+2 a_{5}\right) t^{27}+\cdots
\end{aligned}
$$


Apliquemos o Algoritmo 2.2 para obter uma Base Standard de. módulos para $\mathcal{O} d \mathcal{O}$.

$O S$-processo de menor altura, que neste caso coincide com sua redução final, é

$$
\Omega_{1}=x d y-\frac{3}{2} y d x=t^{15}+2 a_{1} t^{16}+4 a_{2} t^{18}+5 a_{3} t^{19}+7 a_{4} t^{21}+8 a_{5} t^{22}+\cdots,
$$

como $v\left(\Omega_{1}\right)+1=16, v\left(x \Omega_{1}\right)+1=22, v\left(z \Omega_{1}\right)+1=35$ e $v\left(x z \Omega_{1}\right)+1=41$, podemos considerar $l=32$ como lacuna limitante. Dessa forma, os demais $S$-processos a serem analisados no primeiro passo, são:

$$
\begin{aligned}
& 2 y d y-3 x^{2} d x \stackrel{-d z}{\longrightarrow} 0 \\
& x d z-\frac{19}{6} z d x=(\left.+2 a_{1}\right) t^{25}+4 a_{1} t^{26}+\left(3 a_{1}^{2}+6 a_{2}\right) t^{27}+\left(8 a_{2}+8 a_{3}\right) t^{28}+ \\
&+\left(10 a_{1} a_{2}+10 a_{3}\right) t^{29}+\left(12 a_{1} a_{3}+12 a_{4}\right) t^{30}+ \\
&+\left(7 a_{2}^{2}+14 a_{4}+14 a_{5}\right) t^{31}+\cdots \\
& 9 y d z-19 z d y=\left(18 a_{1}-29\right) t^{28}-\left(10+60 a_{1}\right) t^{29}+\left(-31 a_{1}^{2}-31 a_{1}+54 a_{2}\right) t^{30}+ \\
&+\left(-32 a_{1}^{2}-64 a_{2}+72 a_{3}\right) t^{31}+\cdots
\end{aligned}
$$

Agora devemos analisar algumas possibilidades:

1. Se $a_{1} \neq-\frac{1}{2}$, então $\Omega_{2}=x d z-\frac{19}{6} z d x$.

Temos assim, que $v\left(\Omega_{2}\right)+1=26, v\left(x \Omega_{2}\right)+1=32$ el $l=29$ passa $a$ ser a lacuna limitante.

(a) Se $a_{1} \neq \frac{29}{18}$, então $\Omega_{3}=9 y d z-19 z d y, v\left(\Omega_{3}\right)+1=29$ e a lacuna limitante pode ser considerada como $l=23$. Uma rápida análise mostra que não teremos nenhum outro $S$-processo no próximo passo, o que indica o fim do algoritmo.

(b) $S e a_{1}=\frac{29}{18}$, então no próximo passo os $S$-processos com altura inferior a $l=29$, bem como suas reduções finais são:

$2 y \Omega_{1}-\frac{1}{6} z d x \stackrel{-\Omega_{2}}{\longrightarrow} 0$,

$38 y \Omega_{1}-x d z \stackrel{-18 \Omega_{2}}{\longrightarrow} 0$,

$38 x^{2} \Omega_{1}-y d z=-\frac{640}{3} t^{29}+\cdots \quad \stackrel{\left\{d x, d y, d z, \Omega_{1}, \Omega_{2}\right\}_{+}}{\longrightarrow}$

0.

Assim, o algortimo finaliza. 
2. Se $a_{1}=-\frac{1}{2}$, ent.ão $\Omega_{2}=y d z-\frac{19}{9} z d y$, e $v\left(\Omega_{2}\right)+1=29$.

Temos

$$
\begin{aligned}
x d z- & \frac{19}{6} z d x \stackrel{-\frac{1}{3} x^{2} y d x}{\longrightarrow} \stackrel{b z d y}{\longrightarrow} \Omega_{3}:=\left(8 a_{3}-\frac{73}{18}+\frac{4}{3} a_{2}\right) t^{28}+ \\
& +\left(\frac{5}{3} a_{2}+\frac{55}{18}+10 a_{3}\right) t^{29}+\left(-6 a_{3}+\frac{11}{6} a_{2}+\frac{341}{288}+12 a_{4}-6 a_{2}^{2}\right) t^{30}+ \\
& +\left(14 a_{5}-\frac{112}{9} a_{2}-\frac{3}{4} a_{3}-\frac{11}{9}-6 a_{2} a_{3}+14 a_{4}-\frac{43}{3} a_{2}^{2}\right) t^{31}+\cdots \\
\text { com } b & =\frac{1}{18}\left(6 a_{2}+\frac{11}{4}\right) .
\end{aligned}
$$

No próximo passo do algoritmo, os $S$-processos a serem.considerados $e$ suas reduções finais são;

$2 y \Omega_{1}-\frac{1}{6} z d x \stackrel{\frac{1}{3} x^{2} y d x}{\longrightarrow} \stackrel{\frac{b_{1}}{18} z d y}{\longrightarrow} \stackrel{-\Omega_{3}}{\longrightarrow} 0$, onde $b_{1}=\left(6 a_{2}+\frac{11}{4}\right)$,

$38 y \Omega_{1}-x d z \stackrel{6 x^{2} y d x}{\longrightarrow} \stackrel{-\frac{b_{2}}{18} z d y}{\longrightarrow} \stackrel{-18 \Omega_{3}}{\longrightarrow} 0$, onde $b_{2}=\left(108 a_{2}+\frac{99}{2}\right)$,

$38 x^{2} \Omega_{1}-y d z \stackrel{-2 \Omega_{2}}{\longrightarrow} 0$

$-38 \Omega_{3}-\left(8 a_{3}-\frac{73}{18}+\frac{4}{3} a_{2}\right) \Omega_{2} \stackrel{\frac{b_{3}}{6} x^{4} d x}{\longrightarrow} \stackrel{-\frac{b_{4}}{12} x z d x}{\longrightarrow} \Omega_{4}:=A t^{31}+\cdots$,

onde

$b_{3}=90 a_{2}-540 a_{3}-35$,

$b_{4}=156 a_{2}^{2}+166 a_{3}+139 a_{2}-432 a_{2} a_{3}-456 a_{4}-\frac{217}{16} e$

$A=224 a_{2}+14-532 a_{5}+630 a_{2}^{2}-532 a_{4}+644 a_{2} a_{3}-576 a_{2}^{2}+\frac{769}{2} a_{2}$.

Observe ainda, que se $8 a_{3}-\frac{73}{18}+\frac{4}{3} a_{2}=0$, então a redução do último $S$-processo corresponde, na verdade, a redução de, $-38 \Omega_{3}$.

Podemos ter dois casos:

(a) $A \neq 0$.

Nesse caso, $v\left(\Omega_{4}\right)+1=32$ e podemos considerar $l=26$ como a lacuna limitante, o que indica o fim do algoritmo. 
(b) $A=0$.

Vemos que nessa situação não há $S$-processos a serem considerados cuja altura seja inferior a $l=32$. Portanto, finalizamos $o$ algoritmo.

Resumindo:

\begin{tabular}{|c|c|c|c|}
\hline$n_{1} \notin\left\{-\frac{1}{2}, \frac{29}{18}\right\}$ & $\Lambda_{1} \backslash \Gamma=\{16,22,26,29,32,35,41\}$ & $\tau=35$ \\
\hline$a_{1}=\frac{29}{18}$ & $\Lambda_{2} \backslash \Gamma=\{16,22,26,32,35,41\}$ & $\tau=36$ \\
\hline$a_{1}=-\frac{1}{2}$ & $A \neq 0$ & $\Lambda_{3} \backslash \Gamma=\{16,22,29,32,35,41\}$ & $\tau=36$ \\
\cline { 2 - 4 } & $A=0$ & $\Lambda_{4} \backslash \Gamma=\{16,22,29,35,41\}$ & $\tau=37$ \\
\hline
\end{tabular}

Os possiveis diagramas de lacunas são:

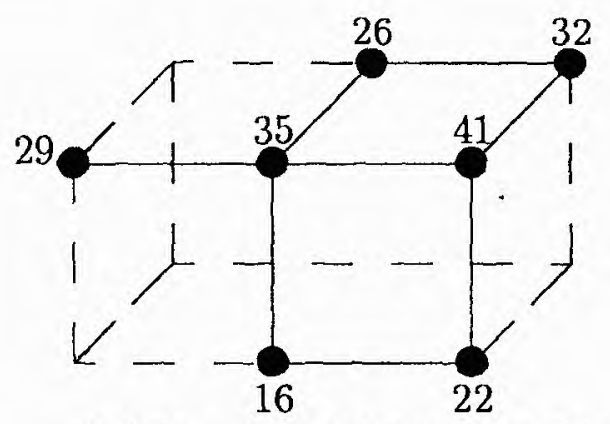

$\Lambda_{1} \backslash \Gamma=\{16,22,26,29,32,35,41\}$

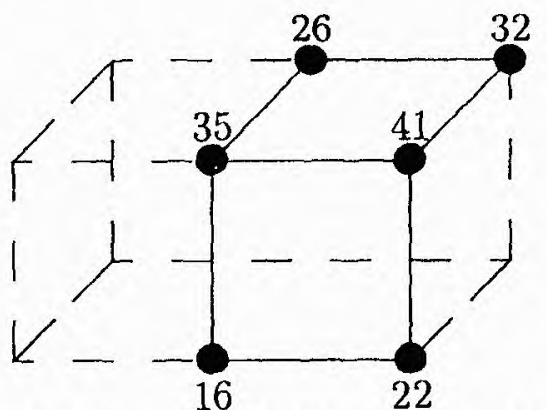

$\Lambda_{2} \backslash \Gamma=\{16,22,26,32,35,41\}$

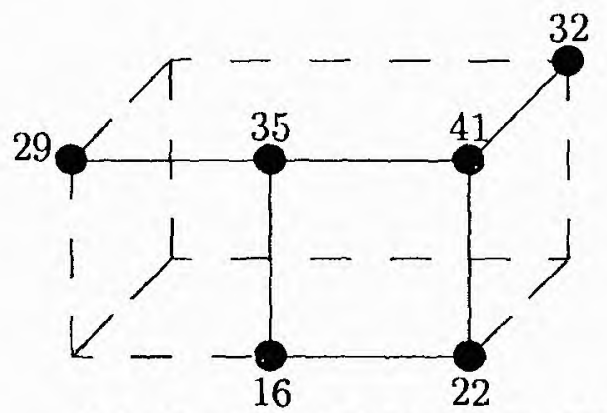

$\Lambda_{3} \backslash \Gamma=\{16,22,29,32,35,41\}$

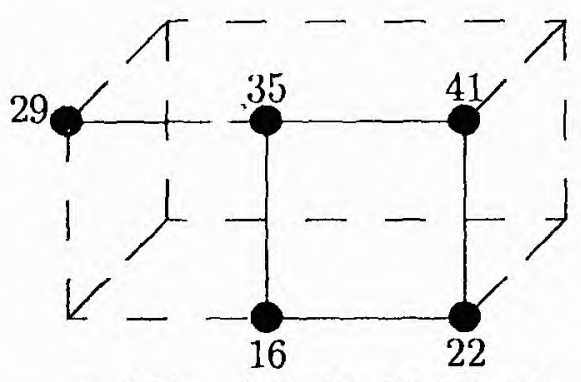

$\Lambda_{4} \backslash \Gamma=\{16,22,29,35,41\}$ 
Observação 3.5 Azevedo em [Az], conjecturou que dado um semigrupo $\Gamma$, $o$ ramo canônico associado $\grave{a} \Gamma$, i.e.,

$$
C:\left\{\begin{array}{l}
x=t^{\beta_{0}} \\
y=t^{\beta_{1}}+\sum_{i=2}^{g} t^{\beta_{i}},
\end{array}\right.
$$

onde $\beta_{0}<\beta_{1}<\cdots<\beta_{g}$ é a seqüiência característica de $\Gamma$ teria número de Tjurina máximo nesta classe de equisingularidade.

Heinrich, apresenta em [He], um contraexemplo a esta conjectura na classe de equisingularidade do exemplo anterior, usando um método distinto do algoritmo aqui apresentado.

O método que Heinrich usa para calcular o número de Tjurina de uma curva nesta classe de equisingularidade, recai no cálculo do posto de uma matriz $40 \times 30$.

No caso do ramo canônico ele encontra o valor $\tau=35$ e por tentativa e. erro, acreditamos, determina a curva

$$
C:\left\{\begin{array}{l}
x=t^{6} \\
y=t^{9}+2 t^{10}-2 t^{11}
\end{array}\right.
$$

cujo número de Tjurina é $\tau=36$, derrubando assim, a conjectura de. Azevedo.

Refaçamos estes cálculos usando a nossa tabela apresentada no exemplo anterior.

Note que o ramo canônico

$$
C_{0}:\left\{\begin{array}{l}
x=t^{6} \\
y=t^{9}+t^{10}
\end{array}\right.
$$

possui $a_{1}=0$, portanto, $\tau=35$, i.e., o mínimo dentre, os possiveis valores de $\tau$ nesta classe de equisingularidade.

Além disso, a curva $C$ de Heinrich, mediante a mudança $t_{1}=\frac{1}{2} t$ e. $y_{1}=$ $\left(\frac{1}{2}\right)^{9} y$ é equivalente à curva

$$
C_{1}:\left\{\begin{array}{l}
x_{1}=t_{1}^{6} \\
y_{1}=t_{1}^{9}+t_{1}^{10}-\frac{1}{2} t_{1}^{11}
\end{array}\right.
$$

Esta curva possui $a_{1}=-\frac{1}{2}$ e $A=14$, o que nos dá $\tau=36$.

No entanto, $C_{1}$ também não atinge o valor máximo para $\tau$. 
Um exemplo de curva que atinge $\tau$ máximo é

$$
C_{M}:\left\{\begin{array}{l}
x=t^{6} \\
y=t^{9}+t^{10}-\frac{1}{2} t^{11}+\frac{1}{38} t^{17} .
\end{array}\right.
$$

A saber, $a_{1}=-\frac{1}{2}$ e $A=0$, portanto $\tau=37$.

Definição 3.4 Dizemos que uma diferencial $\Omega \in \mathcal{O} d \mathcal{O}$ pertence ao $i$-ésimo bloco se $v(\Omega)+1=\sum_{k=1}^{i} s_{k} v_{k}-s_{0} v_{0}$, com $s_{i} \neq 0$.

Note que se $\Omega$ é uma DNE, então $\Omega$ é uma diferencial do $i$-ésimo bloco se, e somente se, o ponto do diagrama de lacunas correspondente à $v(\Omega)+1$ é $\left(\sigma-s_{0}, s_{1}, \ldots, s_{i}, 0, \ldots, 0\right)$, com $s_{i} \neq 0$.

Podemos ordenar os pontos de $\varrho(\Lambda) \subset \mathbb{N}^{g+1}$, onde $\varrho$ é a aplicação definida em (3.1), de modo a preservar a ordem natural dos elementos de $\Lambda \subseteq \mathbb{N}$, como mostra o lema abaixo.

Lema 3.1 Sejam $p$ e $q$ elementos de $\Lambda, A=\varrho(p)$ e $B=\varrho(q)$. Denotando por $\pi_{A}$ e por $\pi_{B}$ os hiperplanos de $\mathbb{Q}^{g+1}$ com vetor normal $\left(v_{0}, v_{1}, \ldots, v_{g}\right)$ que passam por $A$ e $B$ respectivamente, temos que $p<q$ se, e somente se, a interseção de $\pi_{A}$ com qualquer um dos eixos $X_{i}$ é menor que a interseção de $\pi_{B}$ com este. mesmo eixo.

Dem.: As equaçōes dos hiperplanos em $\mathbb{Q}^{+1}$ normais ao vetor $\left(v_{0}, v_{1}, \ldots, v_{g}\right)$ e que passam por $A=\left(\sigma-\alpha_{0}, \alpha_{1}, \ldots, \alpha_{g}\right)$ e por $B=\left(\sigma-\delta_{0}, \delta_{1}, \ldots, \delta_{g}\right)$ são respectivamente:

$$
\begin{gathered}
\pi_{A}:\left(v_{0}, v_{1}, \ldots, v_{g}\right) \cdot\left(X_{0}-\left(\sigma-\alpha_{0}\right), X_{1}-\alpha_{1}, \ldots, X_{g}-\alpha_{g}\right)=0 \\
\sum_{i=0}^{g} v_{i} X_{i}=\sum_{i=1}^{g} \alpha_{i} v_{i}+\left(\sigma-\alpha_{0}\right) v_{0} . \\
\pi_{B}:\left(v_{0}, v_{1}, \ldots, v_{g}\right) \cdot\left(X_{0}-\left(\sigma-\delta_{0}\right), X_{1}-\delta_{1}, \ldots, X_{g}-\delta_{g}\right)=0 \\
\sum_{i=0}^{g} v_{i} X_{i}=\sum_{i=1}^{g} \delta_{i} v_{i}+\left(\sigma-\delta_{0}\right) v_{0} .
\end{gathered}
$$

A interseção de $\pi_{A}$, respectivamente $\pi_{B}$, com o eixo $X_{i}$ é

$$
v_{i}^{-1}\left(\sum_{i=1}^{g} \alpha_{i} v_{i}+\left(\sigma-\alpha_{0}\right) v_{0}\right)
$$


respectivamente.

$$
v_{i}^{-1}\left(\sum_{i=1}^{g} \delta_{i} v_{i}+\left(\sigma-\delta_{0}\right) v_{0}\right)
$$

Desse modo,

$$
\frac{\sum_{i=1}^{g} \alpha_{i} v_{i}+\left(\sigma-\alpha_{0}\right) v_{0}}{v_{i}}<\frac{\sum_{i=1}^{g} \delta_{i} v_{i}+\left(\sigma-\delta_{0}\right) v_{0}}{v_{i}} \Leftrightarrow p<q .
$$

Observação 3.6 Se $\Omega_{i} \in \mathcal{O} d \mathcal{O}$ pertence ao i-ésimo bloco, $\Omega_{j} \in \mathcal{O} d \mathcal{O}$ pertence ao $j$-ésimo bloco e $i<j$, podemos comparar $P_{i}=v\left(\Omega_{i}\right)+1$ com $P_{j}=v\left(\Omega_{j}\right)+1$ analisando a interseção com algum eixo $X_{k}$ onde $0 \leq k \leq j$ com os hiperplanos de $\mathbb{Q}^{j+1}$ que são normais ao vetor $\left(v_{0}, v_{1}, \ldots, v_{j}\right)$ e que passam por $\varrho\left(P_{i}\right)$ e $\varrho\left(P_{j}\right)$.

Vamos analisar o algoritmo para obter uma Base Standard de $\psi(\mathcal{O} d \mathcal{O})$ restrito às diferenciais do primeiro bloco.

Obviamente o algoritmo inicia com $G=\{d x, d y\}$ e a lacuna limitante é $l=\left(n_{1}-1\right) v_{1}-v_{0}$, uma vez que qualquer lacuna superior a $l$ pertence a blocos superiores.

O único $S$-processo mínimo não trivial com altura inferior a $l$ envolvendo $d x$ e $d y$ é $S=x d y-\frac{v_{1}}{v_{0}} y d x$.

Seja $r$ uma redução final de $S$ módulo $(G,\{x, y\})$. Se $r=0$ ou $e_{1} \not$ $(v(r)+1)$, então não temos DNEM no primeiro bloco.

Vamos supor que $\Omega_{1}=r$ é uma DNEM do primeiro bloco. Para o próximo passo devemos déterminar o novo valor para a lacuna limitante e o conjunto dós $S$-processos mínìmos de $G=\left\{d x, d y, \Omega_{1}\right\}$.

Se $v\left(\Omega_{1}\right)+1=\alpha_{1} v_{1}-\delta_{1} v_{0}$, então os $S$-processos a serein considerados são dà forma

$$
\begin{gathered}
S_{1}=x^{\delta_{1}+1} \Omega_{1}-a_{1} y^{\alpha_{1}} d x, \\
S_{2}=x^{\delta_{1}} \Omega_{1}-a_{2} y^{\alpha_{1}-1} d y, \\
S_{3}=y^{n_{1}-\alpha_{1}} \Omega_{1}-a_{3} x^{m_{1}-\delta_{1}-1} d x, \\
S_{4}=y^{n_{1}-\alpha_{1}+1} \Omega_{1}-a_{4} x^{m_{1}-\delta_{1}} \dot{d} y,
\end{gathered}
$$

onde $m_{1}=\frac{v_{1}}{e_{1}}$ e $n_{1}=\frac{v_{0}}{e_{1}} \operatorname{com} e_{1}=\operatorname{MDC}\left(v_{0}, v_{1}\right)$. 
Vamos interpretar os $S$-processos acima, usando a correspondência

$$
\varrho: \begin{array}{ccc}
\Lambda & \longrightarrow & \mathbb{N}^{g+1} \\
\sum_{i=1}^{g} s_{i} v_{i}-s_{0} v_{0} & \mapsto & \left(\sigma-s_{0}, s_{1}, \ldots, s_{g}\right)
\end{array}
$$

$\operatorname{com} 0 \leq s_{i}<n_{i}$ para $i=1, \ldots, g$ e $s_{0}<\sigma$.

Como estamos nos restringindo apenas às diferenciais do primeiro bloco, podemos considerar $s_{0}<m_{1}$.

Assim a correspondência acima, pode ser reescrita como

$$
s_{1} v_{1}-s_{0} v_{0} \mapsto\left(m_{1}-s_{0}, s_{1}\right)
$$

com $0 \leq s_{1}<n_{1}$ e $s_{0}<m_{1}$.

Note que se não fixarmos a representação privilegiada (veja pag. 69) dos elementos de $\Lambda$ que correspondem às diferenciais do primeiro bloco, i.e., $s_{1} v_{1}-s_{0} v_{0} \operatorname{com} 0 \leq s_{1}<n_{1}$ e $s_{0}<m_{1}$, então podemos ter, eventualmente, dois pontos em $\mathbb{N}^{2}$ representando o mesmo elemento de $\Lambda$. De fato,

$$
s_{1} v_{1}-s_{0} v_{0}=0 \Leftrightarrow s_{1} m_{1}=s_{0} n_{1}
$$

e como $\operatorname{MDC}\left(n_{1}, m_{1}\right)=1$, devemos ter $s_{1}=k n_{1}$ e $s_{0}=k m_{1}$. Assim,

$$
0=s_{1} v_{1}-s_{0} v_{0}=k n_{1} v_{1}-k m_{\cdot 1} v_{0} \stackrel{\varrho}{\mapsto}\left((1-k) m_{1}, k n_{1}\right)=O_{k}
$$

Para que $O_{k}$ seja um ponto de $\mathbb{N}^{2}$ devemos ter $k=0$ ou $k=1$. Desse modo, $0 \in \Lambda$ pode ser representado por $O_{0}=\left(m_{1}, 0\right)$ e por $O_{1}=\left(0, n_{1}\right)$. Conseqüentemente, qualquer elemento da forma $\gamma_{1} v_{1}+\gamma_{0} v_{0} \in \Gamma \subseteq \Lambda$, pode ser representado por $\left(m_{1}+\gamma_{0}, \gamma_{1}\right)$ e por $\left(\gamma_{0}, n_{1}+\gamma_{1}\right)$. Observe que $v(d x)+1=v_{0}$ pode ser representado por $A_{0}=\left(m_{1}+1,0\right)$ e por $A_{1}=\left(1, n_{1}\right)$, enquanto que $v(d y)+1=v_{1}$ por $B_{0}=\left(m_{1}, 1\right)$ e por $B_{1}=\left(0, n_{1}+1\right)$.

Note que se $\delta_{1} v_{1}-\delta_{0} v_{0} \in \Lambda \backslash \Gamma$, i.e., $\delta_{0}>0$, então podemos representar tal valor apenas por $\left(m_{1}-\delta_{0}, \delta_{1}\right)$.

Representando $v\left(\Omega_{1}\right)+1=\alpha_{1} v_{1}-\gamma_{1} v_{0}$ por $P_{1}=\left(m_{1}-\gamma_{1}, \alpha_{1}\right)$, vemos que os $S$-processos a serem considerados podem ser obtidos através da interpretação, por meio da associação $\varrho$, dos pontos de interseção da reta horizontal que passa por $P_{1}$ com as retas verticais que passam por $A_{0}$ e $B_{0}$ e dos pontos de interseção da reta vertical que passa por $P_{1}$ com as retas horizontais que passam por $A_{1}$ e $B_{1}$ (Veja a figura abaixo). 


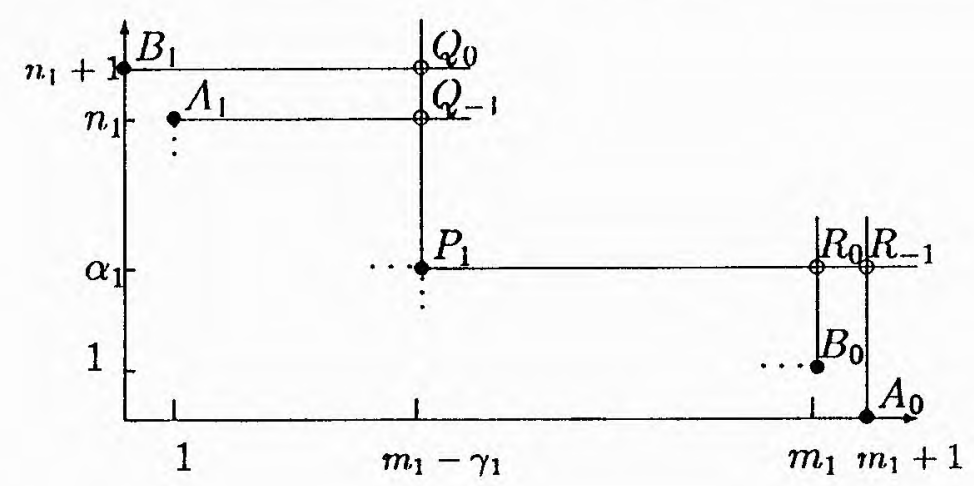

Note que

$$
R_{-1}=\left(m_{1}+1, \alpha_{1}\right)=\left\{\begin{array}{l}
\left(0, \alpha_{1}\right)+\left(m_{1}+1,0\right)=\left(0, \alpha_{1}\right)+A_{0} \\
\left(\gamma_{1}+1,0\right)+\left(m_{1}-\gamma_{1}, \alpha_{1}\right)=\left(\gamma_{1}+1,0\right)+P_{1}
\end{array}\right.
$$

o que equivale a dizer que $v\left(y^{\alpha_{1}} d x\right)=v\left(x^{\gamma_{1}+1} \Omega_{1}\right)$, i.e., temos o $S$-processo $S_{1}$. Do mesmo modo, podemos obter os $S$-processos $S_{2}, S_{3}$ e $S_{4}$ listados anteriormente.

É óbvio que $h t\left(S_{2}\right) \prec h t\left(S_{1}\right)$ e $h t\left(S_{3}\right) \prec h t .\left(S_{4}\right)$, onde $\prec$ é a ordem monomial de $K\left[\left[t_{1}\right]\right.$. Denotemos por $h t_{1}=\min \left\{h t\left(S_{2}\right), h t\left(S_{3}\right)\right\}$ e $h t^{1}=\max \left\{h t\left(S_{2}\right), h t\left(S_{3}\right)\right\}$.

Agora observe que se $l$ é a lacuna limitante neste passo para o algoritmo restrito às diferenciais do primeiro bloco, então $l<v\left(h t^{1}\right)$. De fato, se $Q_{-1}$ representa o $S$-processo com altura $h t_{1}$ e $R_{0}$ representa o $S$-processo de altura $h t^{1}$, então qualquer ponto que se localize no semiplano superior de $\mathbb{N}^{2}$ determinado pela reta de vetor normal $\left(v_{0}, v_{1}\right)$ e que passa por $R_{0}$ representa um elemento de $\left\{v(d x)+1+\left\langle v_{0}, v_{1}\right\rangle\right\} \cup\left\{v(d y)+1+\left\langle v_{0}, v_{1}\right\rangle\right\} \cup\left\{v\left(\Omega_{1}\right)+1+\right.$ $\left.\left\langle v_{0}, v_{1}\right\rangle\right\}$, portanto sua redução módulo $\left(\left\{d x, d y, \Omega_{1}\right\},\{x, y\}\right)$ vão pertence ao primeiro bloco.

- Conseqüentemente, basta computarmos uma redução final dos $S$ processos listados anteriormente, tal que a ordem de sua altura esteja em $v\left(h t_{1}\right)+\left\langle v_{0}, v_{1}\right\rangle$. Além disto, se alguma redução final é uma nova diferencial do primeiro bloco, então o ponto em $\mathbb{N}^{2}$ 'que corresponde a ela, se localiza entre: as retas com vetor normal $\left(v_{0}, v_{1}\right)$ que passam por $Q_{-1}$ e $R_{0}$ e fora da região $\left(A_{0}+\mathbb{N}^{2}\right) \cup\left(A_{1}+\mathbb{N}^{2}\right) \cup\left(B_{0}+\mathbb{N}^{2}\right) \cup\left(B_{1}+\mathbb{N}^{2}\right) \cup\left(P_{1}+\mathbb{N}^{2}\right)$ (Veja figura abaixo). 


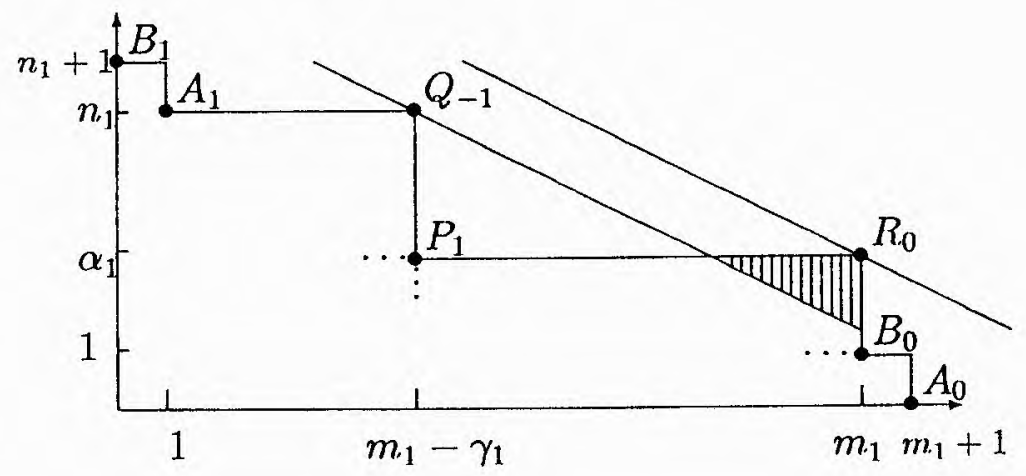

De modo análogo, se $G=\left\{\Omega_{-1}=d x, \Omega_{0}=d y, \Omega_{1}, \ldots, \Omega_{i}\right\}$ representa o conjunto das diferenciais do primeiro bloco, obtidas pelo Algoritmo 2.2, atć um determinado passo, então os $S$-processos envolvendo $\Omega_{j}$ com $1 \leq j \leq i$. são da forma

$$
\begin{aligned}
& x^{\alpha_{k}} \Omega_{j}+a_{k} y^{\gamma_{k}} \Omega_{k} \\
& y^{\delta_{k}} \Omega_{j}+b_{k} x^{\eta_{k}} \Omega_{k},
\end{aligned}
$$

com $k=-1, \ldots, i, k \neq j$ e podem ser obtidos através da interseção da reta horizontal, respectivamente vertical, que passa pelo ponto que representa $v\left(\Omega_{j}\right)+1$ com as retas verticais, respectivamente horizontais, que passam pelos pontos que correspondem à $v\left(\Omega_{k}\right)+1$.

Fixado $1 \leq j \leq i$, defina

$$
\begin{aligned}
h t_{X}= & \text { menor altura de um } S \text {-processo } \\
& \text { da forma } x^{\alpha_{k}} \Omega_{j}+a_{k} y^{\gamma_{k}} \Omega_{k},
\end{aligned}
$$

que será chamado de altura mínima de S-processos de $\Omega_{j}$ na direção $x$.

$$
\begin{aligned}
h . t_{Y}= & \text { menor altura de um } S \text {-processo } \\
& \text { da forma } y^{\delta_{k}} \Omega_{j}+b_{k} \cdot x^{\eta_{k}} \Omega_{k},
\end{aligned}
$$

que será chamado de altura mínima de $S$-processos de $\Omega_{j}$ na direção $y$.

Se $h t_{j}=\min \left\{h t_{X}, h t_{Y}\right\}$ e $h t^{j}=\max \left\{h t_{X}, h t_{Y}\right\}$, então os únicos $S$ processos que eventualmente possuem redução final no primeiro bloco são aqueles cuja ordem de sua altura esteja em $v\left(h t_{j}\right)+\left\langle v_{0}, v_{1}\right\rangle$.

Como antes, se alguma redução final de um $S$-processo envolvendo $\Omega_{j}$ para $1 \leq j \leq i$ é uma nova diferencial do primeiro bloco, então o ponto em $\mathbb{N}^{2}$ correspondente a tal diferencial' se localiza entre as retas de vetor normal $\left(v_{0}, v_{1}\right)$ que passam pelos pontos correspondentes à $v\left(h t_{j}\right)$ e $v\left(h t^{j}\right)$ e fora da 
região $\left(A_{0}+\mathbb{N}^{2}\right) \cup\left(A_{1}+\mathbb{N}^{2}\right) \cup\left(B_{0}+\mathbb{N}^{2}\right) \cup\left(B_{1}+\mathbb{N}^{2}\right) \cup_{j=1}^{i}\left(P_{j}+\mathbb{N}^{2}\right)$, onde $P_{j}$ é o ponto de $\mathbb{N}^{2}$ correspondente à $v\left(\Omega_{j}\right)+1$. Além disso, a lacuna limitante $l$, neste passo é menor que $\min \left\{v\left(h t^{j}\right) ; j=1, \ldots, i\right\}$.

Se $j=i=3$, então um exemplo da situação acima pode ser dado pela figura abaixo, onde aos pontos $Q_{j}$ correspondem $S$-processos mínimos do tipo $y^{\delta_{k}} \Omega_{j}+b_{k} x^{\eta_{k}} \Omega_{k}$ e aos pontos $R_{j}, S$-processos da forma $x^{\alpha_{k}} \Omega_{j}+a_{k} y^{\gamma_{k}} \Omega_{k}$ para $k=-1,0,1,2$.

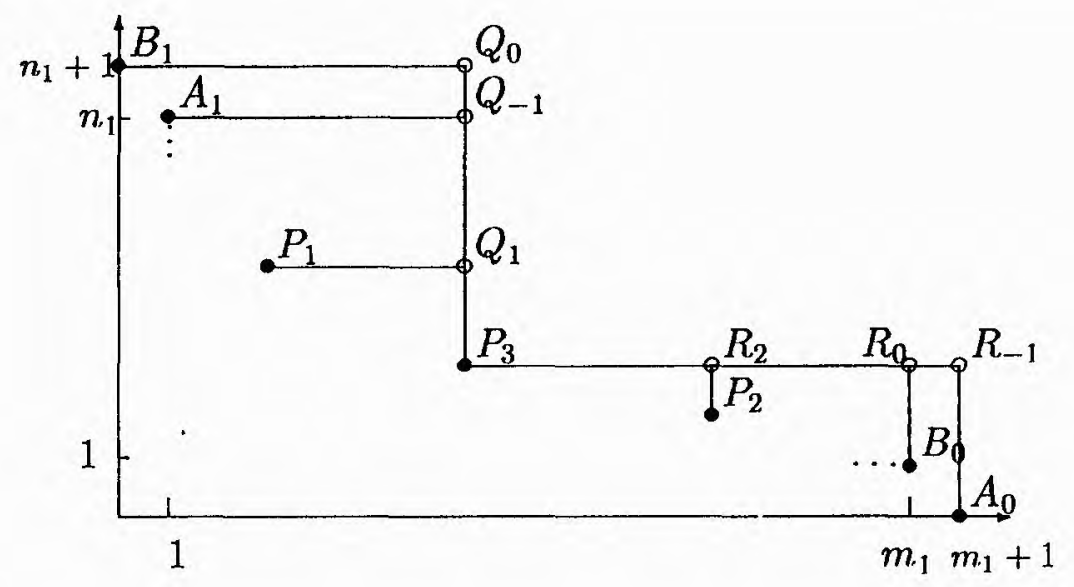

Utilizando os fatos acima podemos obter informações mais refinadas a respeito dos $S$-processos que originam DNEM no primeiro bloco.

O resultado a seguir, foi mostrado por Delorme, em [D2], para curvas de gênero 1 num contexto bem mais aritmético que aqui é reinterpretado à luz do nosso algoritmo.

Proposição 3.3 Sejam $\Omega_{-1}=d x, \Omega_{0}=d y e\left\{\Omega_{1}, \ldots, \Omega_{r}\right\}$ as DNEM do primeiro bloco, com $v\left(\Omega_{i-1}\right)<v\left(\Omega_{i}\right)$ para $i=0, \ldots, r$. Podemos sempre obter uma $D N E M \Omega_{i+1}^{\prime}$ com $v\left(\Omega_{i+1}^{\prime}\right)=v\left(\Omega_{i+1}\right)$ para $0 \leq i<r$ através de uma redução final de um $S$-processo da forma $S\left(\Omega_{i}, \Omega_{j}\right)$, com $-1 \leq j<i e$ $h t\left(S\left(\Omega_{i}, \Omega_{j}\right)\right)=h t_{i}$.

Dem.: A demonstração será feita por indução sobre $i$.

Para $i=0$, o resultado segue diretamente, uma vez que $\Omega_{1}$ é a redução final do $S$-processo $S(d y, d x)=x d y-\frac{v_{1}}{v_{0}} y d x$, cuja altura é $h t_{1}$.

Vamos supor que a proposição seja válida para todo $k \leq i$. 
Sabemos que $\Omega_{i+1}$ é obtida por uma redução final de um $S$-processo da forma $S\left(\Omega_{h}, \Omega_{k}\right)$ módulo $\left(\left\{\Omega_{-1}, \Omega_{0}, \Omega_{1}, \ldots, \Omega_{i}\right\},\{x, y\}\right)$, ou seja,

$$
\Omega_{i+1}=S\left(\Omega_{h}, \Omega_{k}\right)+\sum_{j=-1}^{i} A_{j} \Omega_{j}=\sum_{j=-1}^{i} B_{j} \Omega_{j}
$$

com $A_{j}, B_{j} \in \mathcal{O}$. Note que nenbuma parcela de $\sum_{j=-1}^{i} A_{j} \Omega_{j}$ contribui para a altura da representação (3.2), fato garantido pelo processo de redıção. Note também que na maneira de escrevermos $\Omega_{i+1}$, temos que $B_{j}=A_{j}$ se $j \neq h, k$ e que $v\left(B_{j}\right)=v\left(A_{j}\right)$ para $j=k, h$. Assim, temos

$$
v\left(\Omega_{i+1}\right)>v\left(B_{j} \Omega_{j}\right)>v\left(B_{h} \Omega_{h}\right)=v\left(B_{k} \Omega_{k}\right),
$$

para todo $j=-1, \ldots, i$, com $j \neq h$ e $j \neq k$.

Sem perda de generalidade, podemos supor que $h<k$, i.e., $v\left(\Omega_{h}\right)<$ $v\left(\Omega_{k}\right)$.

Vamos mostrar inicialmente que $k=i$ e depois que $h t\left(S\left(\Omega_{h}, \Omega_{i}\right)\right)=h t_{i}$.

Obviamente $k \leq i$.

Supoula que $k<i$, como vimos anteriormente, podemos considerar $v\left(h t\left(S\left(\Omega_{h}, \Omega_{k}\right)\right)\right) \in v\left(h t_{k}\right)+\left\langle v_{0}, v_{1}\right\rangle$.

Por hipótese de indução temos que

$$
\Omega_{k+1}=S\left(\Omega_{l}, \Omega_{k}\right)+\sum_{j=-1}^{k} C_{j} \Omega_{j}=\sum_{j=-1}^{k} D_{j} \Omega_{j}
$$

$\operatorname{com} C_{j}, D_{j} \in \mathcal{O}$ e

$$
v\left(h t_{k}\right)=v\left(D_{k} \Omega_{k}\right)=\min _{-1 \leq j \leq k}\left\{v\left(D_{j} \Omega_{j}\right)\right\} .
$$

Como $v\left(B_{k} \Omega_{k}\right) \in v\left(h t_{k}\right)+\left\langle v_{0}, v_{1}\right\rangle$, existe $g \in \mathcal{O}$, tal que

$$
v\left(B_{k} \Omega_{k}-g D_{k} \Omega_{k}\right)>v\left(g D_{k} \Omega_{k}\right)=v\left(B_{k} \Omega_{k}\right)
$$

e assim, podemos escrever

$$
\begin{aligned}
\Omega_{i+1} & =\sum_{j=-1}^{i} B_{j} \Omega_{j}=\sum_{j=-1}^{i} B_{j} \Omega_{j}+g\left(\Omega_{k+1}-\sum_{j=-1}^{k} D_{j} \Omega_{j}\right)= \\
& =\sum_{j=-1}^{k}\left(B_{j}-g D_{j}\right) \Omega_{j}+\left(B_{k+1}+g\right) \Omega_{k+1}+\sum_{j=k+2}^{i} B_{j} \Omega_{j} .
\end{aligned}
$$

Agora veja que: 
- $\min _{-1 \leq j<k}\left\{v\left(\left(B_{j}-g D_{j}\right) \Omega_{j}\right)\right\} \geq v\left(B_{k} \Omega_{k}\right)$.

De fato, para todo $j=-1, \ldots, k-1$ temos

$$
\begin{aligned}
& v\left(\left(B_{j}-g D_{s}\right) \Omega_{j}\right) \underset{(3.4)}{\geq \min }\left\{v\left(B_{j} \Omega_{j}\right), v\left(g D_{j} \Omega_{j}\right)\right\} \\
& \geq \min \left\{v\left(B_{j} \Omega_{j}\right), v\left(g D_{k} \Omega_{k}\right)\right\} \\
& \stackrel{(3.5)}{=} \min \left\{v\left(B_{j} \Omega_{j}\right), v\left(B_{k} \Omega_{k}\right)\right\} \\
& \stackrel{(3.3)}{=} v\left(B_{k} \Omega_{k}\right) \text {. }
\end{aligned}
$$

$\bullet v\left(\left(B_{k}-g D_{k}\right) \Omega_{k}\right)>v\left(B_{k} \Omega_{k}\right)$, por (3.5).

- $v\left(\left(B_{k+1}+g\right) \Omega_{k+1}\right)>v\left(B_{k} \Omega_{k}\right)$. De fato, se $v\left(B_{k} \Omega_{k+1}\right) \geq v\left(g \Omega_{k+1}\right)$, então

$$
\begin{aligned}
v\left(\left(B_{k+1}+g\right) \Omega_{k+1}\right) & \geq \min \left\{v\left(B_{k+1} \Omega_{k+1}\right), v\left(g \Omega_{k+1}\right)\right\} \\
& =v\left(g \Omega_{k+1}\right)
\end{aligned}
$$

$$
\begin{aligned}
& \stackrel{(3.4)}{>} v\left(g D_{k} \Omega_{k}\right) \\
& \stackrel{(3.5)}{=} v\left(B_{k} \Omega_{k}\right) .
\end{aligned}
$$

Se $v\left(B_{k+1} \Omega_{k+1}\right)<v\left(g \Omega_{k+1}\right)$, então como $v\left(g \Omega_{k+1}\right)>v\left(g D_{k} \Omega_{k}\right)$, podemos ter:

a) $v\left(B_{k+1} \Omega_{k+1}\right)>v\left(g D_{k} \Omega_{k}\right), \operatorname{assim} \min \left\{v\left(B_{k+1} \Omega_{k+1}\right), v\left(g \Omega_{k+1}\right)\right\}>$ $v\left(g D_{k} \Omega_{k}\right)=v\left(B_{k} \Omega_{k}\right)$.

b) $v\left(B_{k+1} \Omega_{k+1}\right) \leq v\left(g D_{k} \Omega_{k}\right)$. Dessa forma, temos

$$
v\left(B_{k} \Omega_{k}\right) \stackrel{(3.5)}{=} v\left(g D_{k} \Omega_{k}\right) \geq v\left(B_{k+1} \Omega_{k+1}\right)
$$

que contradiz (3.3).

- $\min _{k+1<j \leq i}\left\{v\left(B_{j} \Omega_{j}\right)\right\}>v\left(B_{k} \Omega_{k}\right)$, uma vez que estamos supondo $h .<k$ e os únicos termos que contribuem para a altura de $\Omega_{i+1}$ na representação (3.6) são $B_{h} \Omega_{h}$ e $B_{k} \Omega_{k}$.

Desse modo, temos uma representação para $\Omega_{i+1}$ cuja altura do $S$ processo que a origina é maior on igual a $h t .=h t\left(S\left(\Omega_{h}, \Omega_{k}\right)\right)=v\left(B_{k} \Omega_{k}\right)$.

Podemos ter: 
1. A altura de $\Omega_{i+1}$ em (3.6) é maior que h.t.

Neste caso duas possibilidades podem ocorrer:

(a) $O$ maior índice para as diferenciais envolvidas no $S$-processo que origina $\Omega_{i+1}$ é maior que $k$, i.e., $\Omega_{i+1}$ é obtida pela redução final de $11 \mathrm{~m} S$-processo da forma $S\left(\Omega_{l}, \Omega_{j}\right)$, com $j>k$. Se $j=i$ a primeira afirmação da proposição está provada. Caso contrário, aplicamos o mesmo raciocínio usado para $\Omega_{k+1}$ para a diferencial $\Omega_{j+1}$.

(b) O maior índice para as diferenciais envolvidas no $S$-processo que origina $\Omega_{i+1}$ é menor on igual a $k$, i.e., $\Omega_{i+1}$ é obtida pela redução final de um $S$-processo da forma $S\left(\Omega_{l}, \Omega_{j}\right)$ com $l<j \leq k$. Nesse caso, aplicamos o procedimento para $\Omega_{j+1}$ ao invés de $\Omega_{k+1}$. Note que esse caso não pode ocorrer indefinidamente, pois se $j=k$ para um número infinito de passos, então aumentaremos a altura do $S$-processo de modo a superar $v\left(\Omega_{i+1}\right)$, um absurdo. Por outro lado, se $j<k$, também não podemos proceder indefinidamente. pois $j \geq-1$.

2. A altura de $\Omega_{i+1}$ em (3.6) é igual a $h t$.

Nesto caso, o maior índice para uma diferencial que contribui para a altura de (3.6) é menor do que $k$. Assim, aplicamos o procedimento anterior para o referido índice. Como o índice não pode diminuir indefiuidanıeutce, estaremos após algımas aplicações desse raciocínio na situação 1.a).

Note que a possibilidade 1.a), indica que o raciocínio usado, implica que obrigatoriamente elevaremos o maior índice da diferencial envolvida no $S$ processo que origina $\Omega_{i+1}$. Desse modo, após um número finito de passos do argumento acima teremos

$$
\Omega_{i+1}=S\left(\Omega_{i}, \Omega_{h}\right)+\sum_{j=-1}^{i} E_{j} \Omega_{j}=\sum_{j=-1}^{i} F_{j} \Omega_{j},
$$

$\operatorname{com} E_{j}, F_{j} \in \mathcal{O}, h<i$ e $v\left(h t\left(S\left(\Omega_{i}, \Omega_{h}\right)\right)\right) \in v\left(h t_{i}\right)+\left\langle v_{0}, v_{1}\right\rangle$

Assim, em cada passo do algoritmo, determinamos uma nova DNEM no primeiro bloco, obtida por uma redução final de um $S$-processo envolvendo a DNEM determinada no passo anterior do algoritmo. 
Resta mostrar que podemos tomar o $S$-processo envolvendo $\Omega_{i}$ com mouor altura, i.e., $h t_{i}$.

Inicialmente, notemos que se $\omega_{i+1}$ é uma redução final de $S\left(\Omega_{i}, \Omega_{l}\right)$, onde este $S$-processo é o de menor altura envolvendo $\Omega_{i}$, èntão $v\left(\omega_{i+1}\right) \geq v\left(\Omega_{i+1}\right)$. De fato, se $v\left(\omega_{i+1}\right)<v\left(\Omega_{i+1}\right)$, então $v\left(\omega_{i+1}\right) \in v\left(\Omega_{j}\right)+\left\langle v_{0}, v_{1}\right\rangle$ com $j \leq i$, mas deste modo $\omega_{i+1}$ não seria redução final. Lembre que estamos supoudo que não existe DNEM, no primeiro bloco, cuja ordem esteja entre $v\left(\Omega_{i}\right)$ e $v\left(\Omega_{i+1}\right)$.

Observemos que se a ordem da altura do $S$-processo em (3.7) for $v\left(h t_{i}\right)$ ou $v\left(\omega_{i+1}\right)=v\left(\Omega_{i+1}\right)$, então mostramos que existe uma DNEM que é obtida por uma redução final do $S$-processo de menor altura envolvendo $\Omega_{i}$ e cuja ordem é $v\left(\Omega_{i+1}\right)$.

Podemos supor que na representação (3.7) tenhamos

$$
h t\left(S\left(\Omega_{i}, \Omega_{h}\right)\right)=v\left(F_{i} \Omega_{i}\right)>v\left(h t_{i}\right) \text { e } v\left(\omega_{i+1}\right)>v\left(\Omega_{i+1}\right) .
$$

Seja $\Omega_{i+1}^{\prime}=\Omega_{i+1}+\omega_{i+1}$, note que $v\left(\Omega_{i+1}^{\prime}\right)=v\left(\Omega_{i+1}\right)$. Se $\omega_{i+1}=$ $\sum_{j=-1}^{i} G_{j} \Omega_{j} \operatorname{com} v\left(h t\left(\sum_{j=-1}^{i} G_{j} \Omega_{j}\right)\right)=v\left(G_{i} \Omega_{i}\right)=v\left(h t_{i}\right)$, então para cada $j=-1, \ldots, i$ temos

$$
\begin{aligned}
v\left(\left(F_{j}+G_{j}\right) \Omega_{j}\right) & \geq \min \left\{v\left(F_{j} \Omega_{j}\right), v\left(G_{j} \Omega_{j}\right)\right\} \\
& \geq \min \left\{v\left(F_{i} \Omega_{i}\right), v\left(G_{i} \Omega_{i}\right)\right\} \\
& =\min \left\{v\left(F_{i} \Omega_{i}\right), v\left(h t_{i}\right)\right\} \\
& =v\left(h t_{i}\right)=v\left(\left(F_{i}+G_{i}\right) \Omega_{i}\right) .
\end{aligned}
$$

Portanto, podemos considerar $\Omega_{i+1}^{\prime}$ no lugar de $\Omega_{i+1}$.

Apresentamos a seguir alguns resultados que serão utilizados no Apêndice $A$ e por estarem vinculados aos conceitos que introduzimos anteriormente justificam sua ocorrência neste momento.

Com as notações da proposição anterior, defina $v\left(G^{i}\right)=v\left(h t_{i}\right)-v\left(\Omega_{i}\right)$, para todo $i=0, \ldots, r$ e $\Omega_{i}$ diferencial do primeiro bloco. Note que $v\left(G^{i}\right)>0$ para todo $i=0, \ldots, r$.

Lema 3.2 Escreva $\Omega_{i}=q_{i} d x-p_{i} d y, \operatorname{com} q_{i}, p_{i} \in \mathcal{O}$ para todo $i=1, \ldots, r e$ $\Omega_{i} D N E M$ do primeiro bloco. Então

$$
v\left(p_{i+1}\right)=v\left(p_{i}\right)+v\left(G^{i}\right)
$$


para todo $i=0, \ldots, r-1$, onde, $v\left(p_{0}\right)=0$.

Dem.: A demonstração será feita por indução sobre $i$.

Para $i=0$, temos que $\Omega_{i}=\left(\frac{v_{1}}{v_{0}} y+\cdots\right) d x-(x+\cdots) d y$, i.e., $v\left(p_{1}\right)=$ $v(x)=v_{0}$. Como $v\left(p_{0}\right)=0$ e $v\left(G^{0}\right)=v\left(h t_{0}\right)-v\left(\Omega_{0}\right)=v(x d y)-v(d y)=v(x)$, segue que

$$
v\left(p_{1}\right)=v\left(p_{0}\right)+v\left(G^{0}\right)
$$

Vamos supor que o lema seja verdadeiro para todo $j$, tal que $0 \leq j \leq i<$ $r$.

Considerando $\Omega_{i+1}$ como na proposição anterior, i.e.,

$$
\Omega_{i+1}=\sum_{l=-1}^{i} B_{l} \Omega_{l}
$$

com $B_{l} \in \mathcal{O}$ para todo $l=-1, \ldots, i$ e $v\left(B_{i} \Omega_{i}\right)=v\left(B_{k} \Omega_{k}\right)<v\left(B_{h} \Omega_{h}\right)$, para todo $-1 \leq h<i$ com $h \neq k$.

Como $p_{i+1}=\sum_{l=0}^{i} B_{l} p_{l}$ e $v\left(B_{i}\right)=v\left(G^{i}\right)$, basta provar que $v\left(p_{i}\right)+v\left(B_{i}\right)<$ $v\left(p_{h}\right)+b\left(B_{h}\right)$ para todo $0 \leq h<i$.

Pela expressão de $\Omega_{i+1}$ considerada, temos que

$$
v\left(B_{i}\right)+v\left(\Omega_{i}\right) \leq v\left(B_{h}\right)+v\left(\Omega_{h}\right),
$$

equivalentemente,

$$
v\left(\Omega_{i}\right)-v\left(\Omega_{h}\right)+v\left(p_{h}\right)-v\left(p_{i}\right) \leq v\left(B_{h}\right)-v\left(B_{i}\right)+v\left(p_{h}\right)-v\left(p_{i}\right) .
$$

Por lipótese de indução, temos que

$$
v\left(p_{i}\right)-v\left(p_{h}\right)=\sum_{l=h}^{i-1} v\left(G^{l}\right)=\sum_{l=h}^{i-1} v\left(h t_{i}\right)-v\left(\Omega_{i}\right) .
$$

Assim,

$$
v\left(p_{h}\right)-v\left(p_{i}\right)+v\left(\Omega_{i}\right)-v\left(\Omega_{h}\right)=\sum_{l=h}^{i-1} v\left(\Omega_{l+1}\right)-v\left(h t_{l}\right)>0,
$$

conseqüentemente, por $(*)$, concluímos que

$$
v\left(p_{i}\right)+v\left(B_{i}\right)<v\left(p_{h}\right)+v\left(B_{h}\right) .
$$


Observação 3.7 Seja $\Omega=\sum_{j=-1}^{i} H_{j} \Omega_{j}$, onde $H_{j} \in \mathcal{O}, \Omega_{j}$ é diferencial do primeiro bloco e

$$
v(h t,(\Omega))=v\left(H_{i} \Omega_{i}\right)=v\left(H_{k} \Omega_{k}\right)<v\left(H_{j} \Omega_{j}\right)
$$

para todo $-1 \leq j<i$ com $j \neq k$. Então

$$
v\left(p_{i}\right)+v\left(H_{i}\right)<v\left(p_{j}\right)+v\left(H_{j}\right)
$$

para todo $0 \leq j<i$.

De fato, uma vez que $v\left(H_{i}\right)+v\left(\Omega_{i}\right) \leq v\left(H_{j}\right)+v\left(\Omega_{j}\right)$ e como no lema anterior tem-se que $v\left(p_{j}\right)-v\left(p_{i}\right)+v\left(\Omega_{i}\right)-v\left(\Omega_{j}\right)>0$, concluímos que $v\left(p_{j}\right)-$ $v\left(p_{i}\right)+v\left(H_{j}\right)-v\left(H_{i}\right)>0$.

Obseme ainda que.

$$
v\left(B_{i}\right)+v\left(p_{i}\right)<v\left(B_{-1}\right)+v_{0}-v_{1}
$$

para todo $i \geq 0$. De fato, como $v\left(\Omega_{i}\right)>v\left(h t_{i}\right)=v\left(p_{i}\right)+v_{1}-1$, temos que $v\left(B_{i}\right)+v\left(p_{i}\right)+v_{1}-1<v\left(B_{i}\right)+v\left(\Omega_{i}\right) \leq v\left(B_{-1}\right)+v\left(\Omega_{-1}\right)=v\left(B_{-1}\right)+v_{0}-1$, para todo $i \geq 0$. Portanto, $v\left(B_{i}\right)+v\left(p_{i}\right)<v\left(B_{-1}\right)+v_{0}-v_{1}$. 


\section{Capítulo 4}

\section{Eliminação de Parâmetros}

Uma questão importante no estudo da relação de equivalência de curvas algebróides irredutíveis é obter formas normais que representem todas as curvas com determinados invariantes fixados e que sejam dadas pelo menor número de parâmetros.

Dada uma parametrização de um ramo cujo semigrupo de valores seja $\Gamma=\left\langle v_{0}, v_{1}, \ldots, v_{g}\right\rangle$, vimos (Exemplo 3.2) que todos os termos cujos expoentes $l$ pertencem ao conjunto $\Gamma$ ou ao conjunto $\Gamma+v_{1}-v_{0}$ podem ser eliminados, sem que alteremos os termos de ordem inferior, respectivamente por meio de uma mudança da forma

$$
\begin{aligned}
& t_{1}=t \\
& y_{1}=y+q
\end{aligned}
$$

$\operatorname{com} q \in \mathcal{O}$ e $v(q)=l$ ou

$$
\begin{aligned}
& t_{1}=t\left(1+\frac{p}{t^{v_{0}}}\right)^{\frac{1}{v_{0}}} \\
& y_{1}=y
\end{aligned}
$$

com $p \in \mathcal{O}$ e $v(p)=l-v_{1}+v_{0}$ se $l \in \Gamma+v_{1}-v_{0}$. Ebey, em [E], já observa estas eliminações em casos particulares, enquanto que Zariski, em [Z2] e Azevedo, em $[\mathrm{Az}]$, as apresentam como descritas acima.

A situação anterior é um caso particular do seguinte lema, mostrado por Delorme, em [D2].

Lema 4.1 Seja C uma curva algebróide irredutivel plana dada por

$$
\left\{\begin{array}{l}
x=t^{v_{0}} \\
y=t^{v_{1}}+\sum_{i>v_{i}} a_{i} t^{i}
\end{array}\right.
$$


Se $a_{l} \neq 0$ e existe $\Omega=q d x-p d y \in \mathcal{O} d \mathcal{O}$ tal que $v\left(\frac{\Omega}{d x}\right)=l<2 v(p)-$ $2 v_{0}+v_{1}$, entäo $C$ é equivalente $a$

$$
\left\{\begin{array}{l}
x=t^{v_{0}} \\
y=t^{v_{1}}+\sum_{i>v_{1}}^{l-1} a_{i} t^{i}+\sum_{i>l} a_{i}^{\prime} t^{i}
\end{array}\right.
$$

Dem.: Seja $\Omega=q d x-p d y \in \mathcal{O} d \mathcal{O}$ tal que $\Omega=\left(b t^{l+v_{0}-1}+\cdots\right) d t$ e considere a mudança

$$
\begin{gathered}
t_{1}=t\left(1+\frac{c p}{t^{v_{0}}}\right)^{\frac{1}{v_{0}}} \\
y_{1}=y_{1}+c q .
\end{gathered}
$$

Como já mencionamos no capítulo auterior (Veja Exemplo 3.2) a ação dessa mudança é dada por

$$
\left\{\begin{array}{l}
x_{1}=t_{1}^{v_{0}} \\
y_{1}=t_{1}^{v_{1}}+\sum_{i>v_{1}} a_{i} t_{1}^{i}+\frac{c(q d x-p d y)}{d x} \bmod t^{2 v(p)-2 v_{0}+v_{1}} .
\end{array}\right.
$$

U'ma vez que $t_{1}=t \bmod t^{2}$, podemos escolher $c=-\frac{a_{l} v_{0}}{b}$ e teremos

$$
\left\{\begin{array}{l}
x_{1}=t_{1}^{v_{0}} \\
y_{1}=t_{1}^{v_{1}}+\sum_{i>v_{1}}^{l-1} a_{i} t_{1}^{i}+\sum_{i>l} a_{i}^{\prime} t_{1}^{i}
\end{array}\right.
$$

Seja $F \mp\left\{h_{0}, h_{1}, \ldots, h_{g}\right\}$ uma Base Standard Mínima para $\mathcal{O}$, podemos reescrever o conjunto das potências elimináveis $\Gamma \cup\left(\Gamma+v_{1}-v_{0}\right)$ como

$$
\cup_{i=0}^{1}\left(\Gamma+v_{i}-v_{0}\right)=\cup_{i=0}^{1}\left(\Gamma+v\left(d h_{i}\right)-v\left(d h_{0}\right)\right) .
$$

Escrito desse modo, é natural pensar na possibilidade de ampliar o conjunto das potências elimináveis como segue:

$$
\cup_{i=0}^{g}\left(\Gamma+v\left(d h_{i}\right)-v\left(d h_{0}\right)\right)
$$

Uma vez que a mudança de coordenadas

$$
\begin{aligned}
& t_{1}=t\left(1+\frac{c p}{t^{v_{0}}}\right)^{\frac{1}{v_{0}}} \\
& y_{1}=y+c q,
\end{aligned}
$$


$\operatorname{com} c \in K^{*}$ e. $p, q \in \mathcal{O}$, nos fornece

$$
\left\{\begin{array}{l}
x_{1}=t_{1}^{v_{0}} \\
y_{1}=t_{1}^{v_{1}}+\sum_{i>v_{1}} a_{i} t_{1}^{i}+\frac{c(q d x-p d y)}{d x}-\sum_{i \geq v_{1}} a_{i} t^{i}\left(\sum_{j=2}^{\infty}\left(\begin{array}{c}
\frac{i}{v_{0}} \\
j
\end{array}\right)\left(\frac{c p}{v^{\nu_{0}}}\right)^{j}\right) .
\end{array}\right.
$$

Se considerarmos $p$ e $q$ tais que $F^{\alpha} d h_{i}=q d x-p d y$, então podemos escolher $u \mathrm{~m}$ valor para $c$ de modo que $\frac{c(q d x-p d y)}{d x}=-a_{l} t^{l}+\cdots$, com $l=$ $v\left(F^{\alpha} d h_{i}\right)-v(d x)$. No entanto, nada nos assegura que os termos de ordem inferior a $l$ fiquem inalterados, uma vez que a ordem do segundo somatório em (4.2) pode ser inferior à $l$.

Por exemplo, seja

$$
C:\left\{\begin{array}{l}
x=t^{4} \\
y=t^{10}+t^{17}+a t^{23}
\end{array}\right.
$$

Aplicando a Proposição 2.1, temos que uma Base Standard Míninıa para $\mathcal{O}$ é $F=\{x, y, z\}$ com $z=y^{2}-x^{5}=2 t^{27}+2 a t^{33}+a t^{34}+2 a t^{40}+a^{2} t^{46}$.

Note agora que $d z=-5 x^{4} d x+2 y d y=q d x-p d y$ e $v(d z)-v(d x)=23>$ $22=2 v(p)-2 v_{0}+v_{1}$ contrariamente a hipótese do Lema 4.1. Assim, uma mudança da forma

$$
\begin{aligned}
& t_{1}=t\left(1+\frac{c p}{t^{v_{0}}}\right)^{\frac{1}{v_{0}}} \\
& y_{1}=y+c q,
\end{aligned}
$$

não eliminaria o termo $a t^{23}$ sem que introduzíssemos um termo de ordem 22. No entanto, note que $22=v\left(x^{3} y\right)$, assim podemos eliminar o termo indesejadamente introduzido. Desse modo, compondo a mudança (4.3) com uma da forma (4.1) temos uma mudança de coordenadas, que permite eliminar o termo em questão sem que alteremos os termos anteriores.

Vamos mostrar que o fato acima, não é algo restrito a este exemplo, $e$ sim um fato geral. Para isso, seja $\Gamma=\left\langle v_{0}, v_{1}, \ldots, v_{g}\right\rangle$ e considere uma Base Standard Mínima $F=\left\{h_{0}, h_{1}, \ldots, h_{g}\right\}$ dada pela Proposição 2.1.

No que segue usaremos livremente as notações e relações apresentadas na Subseção 2.1.1 referente a Curvas Planas.

Lembremos (Proposição 2.1) que

$$
h_{i+1}=a_{i} h_{i}^{n_{i}}-b_{i} \prod_{k=0}^{i-1} h_{k}^{\alpha_{k}}-\sum_{j \in J} c_{j} \prod_{k=0}^{i} h_{k}^{\beta_{k, j}}
$$


com

$$
v\left(\sum_{j \in J} c_{j} \prod_{k=0}^{i} h_{k}^{\beta_{k, j}}\right)>v\left(h_{i}^{n_{i}}\right)=v\left(\prod_{k=0}^{i-1} h_{k}^{\alpha_{k}}\right),
$$

com $a_{i}, b_{i}, c_{j} \in K$ convenientes.

Vamos expressar $d h_{i+1}$ em termos de $d x$ e $d y$, on seja, escrever $d h_{i+1}=$ $q_{i+1} d x-p_{i+1} d y$. O primeiro passo é avaliar $v\left(p_{i+1}\right)$ para $i=1, \ldots, g-1$.

Proposição 4.1 Considere as funções $h_{i+1}$ com $i=1, \ldots, g-1$ como na Proposição 2.1. Então tem.os $d h_{i+1}=q_{i+1} d x-p_{i+1} d y \operatorname{com} v\left(p_{i+1}\right)=v\left(p_{i}\right)+$ $\left(n_{i}-1\right) v_{i}$ para $i=1, \ldots, g-1$ e $v\left(p_{1}\right)=0$, ou seja,

$$
v\left(p_{i+1}\right)=\sum_{j=1}^{i}\left(n_{i j}-1\right) v_{j}
$$

Dem.: A demoustração scrá feita por indução sobre $i$.

Para $i=1$, temos de (4.4)

$$
h_{2}=a_{1} y^{n_{1}}-b_{1} x^{\alpha_{0}}-\sum_{j \in J} c_{j} x^{\beta_{j}} y^{\delta_{j}}
$$

com

$$
v\left(\sum_{j \in J} c_{j} x^{\beta_{j}} y^{\delta_{j}}\right)>v\left(y^{n_{1}}\right)=v\left(x^{\alpha_{0}}\right)
$$

Assim,

$$
d h_{2}=q_{2} d x+\left(n_{1} a_{1} y^{n_{1}-1}-\sum_{j \in J} c_{j} \delta_{j} x^{\beta_{j}} y^{\delta_{j}-1}\right) d y
$$

Uma vez que $v\left(x^{\beta_{j}} y^{\delta_{j}}\right)>v\left(y^{n_{1}}\right)$ para todo $j \in J$, segue que

$$
v\left(p_{2}\right)=v\left(n_{1} a_{1} y^{n_{1}-1}-\sum_{j \in J} c_{j} \delta_{j} x^{\beta_{j}} y^{\delta_{j}-1}\right)=\left(n_{1}-1\right) v_{1}
$$

o que garante o resultado para $i=1$.

Vamos supor que a proposição seja válida para todo $k$ tal que $1 \leq k \leq$ $i<g-1$. 
Como, de (4.4)

$$
h_{i+1}=a_{i} h_{i}^{n_{i}}-b_{i} \prod_{k=0}^{i-1} h_{k}^{\alpha_{k}}-\sum_{j \in J} c_{j} \prod_{k=0}^{i} h_{k}^{\beta_{k_{j}}},
$$

e pela hipótese de indução $d h_{k}=q_{k} d x-p_{k} d y$ para todo $1 \leq k \leq i$, temos que

$$
\begin{gathered}
d h_{i+1}=n_{i} a_{i} h_{i}^{n_{i}-1} d h_{i}-b_{i} \sum_{m=0}^{i-1} \alpha_{m}\left(\prod_{k=0, k \neq m}^{i-1} h_{k}^{\alpha_{k}}\right) h_{m}^{\alpha_{m}-1} d h_{m}- \\
-\sum_{j \in J} c_{j} \sum_{m=0}^{i} \beta_{m_{j}}\left(\prod_{k=0, k \neq m}^{i} h_{k}^{\beta_{k_{j}}}\right) h_{m}^{\beta_{m_{j}}-1} d h_{m}= \\
=q_{i+1} d x+\left(n_{i} a_{i} h_{i}^{n_{i}-1} p_{i}-b_{i} \sum_{m=0}^{i-1} \alpha_{m}\left(\prod_{k=0, k \neq m}^{i-1} h_{k}^{\boldsymbol{\alpha}_{k}}\right) h_{m}^{\boldsymbol{\alpha}_{m}-1} p_{m}-\right. \\
\left.-\sum_{j \in J} c_{j} \sum_{m=0}^{i} \beta_{m_{j}}\left(\prod_{k=0, k \neq m}^{i} h_{k}^{\beta_{k_{j}}}\right) h_{m}^{\beta_{m_{j}-1}} p_{m}\right) d y= \\
=q_{i+1} d x-p_{i+1} d y .
\end{gathered}
$$

Note que

$$
\begin{aligned}
& v\left(n_{i} a_{i} h_{i}^{n_{2}-1} p_{i}-\right.\left.b_{i} \sum_{m=0}^{i-1} \alpha_{m}\left(\prod_{k=0, k \neq m}^{i-1} h_{k}^{\alpha_{k}}\right) h_{m}^{\alpha_{m}-1} p_{m}\right) \leq \\
& \leq v\left(\sum_{j \in J} c_{j} \sum_{m=0}^{i} \beta_{m_{j}}\left(\prod_{k=0, k \neq m}^{i} h_{k}^{\beta_{k_{j}}}\right) h_{m}^{\beta_{m_{j}}-1} p_{m}\right) .
\end{aligned}
$$

Agora veja que

$$
\begin{aligned}
v\left(\sum_{m=0}^{i-1} \alpha_{m}\right. & \left.\left(\prod_{k=0, k \neq m}^{i-1} h_{k}^{\alpha_{k}}\right) h_{m}^{\alpha_{m}-1} p_{m}\right) \geq \\
& \geq \min _{0 \leq m<i}\left\{v\left(\left(\prod_{k=0, k \neq m}^{i-1} h_{k}^{\alpha_{k}}\right) h_{m}^{\alpha_{m}-1} p_{m}\right)\right\}= \\
& =\min _{0 \leq m<i}\left\{\sum_{k=0, k \neq m}^{i-1} \alpha_{k} v_{k}+\left(\alpha_{m}-1\right) v_{m}+v\left(p_{m}\right)\right\}= \\
& =\min _{0 \leq m<i}\left\{n_{i} v_{i}-v_{m}+v\left(p_{m}\right)\right\}>n_{i} v_{i}-v_{i}+v\left(p_{i}\right)=v\left(h_{i}^{n_{1}-1} p_{i}\right),
\end{aligned}
$$

lembrando que $\sum_{k=0}^{i-1} \alpha_{k} v_{k}=n_{i} v_{i}$, pois $a_{i} h_{i}^{n_{i}}-b_{i} \prod_{k=0}^{i-1} h_{k}^{\alpha_{k}}$ é um $S$-processo, e notanto que pela hipótese de indução a seqüêucia $v\left(p_{m}\right)-v_{m}$ é crescente para $0 \leq m \leq i$. 
Portanto,

$$
v\left(p_{i+1}\right)=v\left(n_{i} a_{i} h_{i}^{n_{i}-1} p_{i}\right)=\left(n_{i}-1\right) v_{i}+v\left(p_{i}\right)=\sum_{j=0}^{i}\left(n_{j}-1\right) v_{j}
$$

Proposição 4.2 Seja C uma curva algebróide irredutível plana dada por

$$
\left\{\begin{array}{l}
x=t^{v_{0}} \\
y=t^{v_{1}}+\sum_{l>v_{1}} a_{l} t^{l} .
\end{array}\right.
$$

Se $\Gamma=\left\langle v_{0}, v_{1}, \ldots, v_{g}\right\rangle$ é o semigrupo de $C$, então $C$ é equivalente a uma curva dada por

com $a_{l}^{\prime}=0$ para todo $\dot{l} \in \cup_{j=0}^{g}\left(\Gamma+v_{j}-v_{0}\right)$.

$$
\left\{\begin{array}{l}
x=t^{v_{0}} \\
y=t^{v_{1}}+\sum_{l>v_{1}} a_{l}^{\prime} t^{l}
\end{array}\right.
$$

Dèm.: Basta mostrarmos que se $a_{l} t^{l} \operatorname{com} l \in \cup_{j=0}^{g .}\left(\Gamma+v_{j}-v_{0}\right)$ é tal que e $a_{\iota} \neq 0$, então existe uma mudança de coordenadas de modo que a curva $C$ é equivalente a uma curva dada por

$$
\left\{\begin{array}{l}
x=t^{v_{0}} \\
y=t^{v_{1}}+\sum_{i>v_{1}}^{l-1} a_{i} t^{i}+\sum_{i>l} a_{i}^{\prime} t^{i}
\end{array}\right.
$$

Note que para $l \in\left(\Gamma+v_{0}-v_{0}\right) \cup\left(\Gamma+v_{1}-v_{0}\right)$ sabemos ser válida a proposição. Assim, podemos supor que $l=\gamma+v_{i+1}-v_{0}$ para algum $i=1, \ldots, g-1$ e $\gamma \in \Gamma$. Considere $h_{i+1}$ como na Proposição 2.1 e escreva $d h_{i+1}=q_{i+1} d x-p_{i+1} d y$.

Tomemos $h \in \mathcal{O}$ tal que $v(h)=\gamma$, assim podemos escrever $h d h_{i+1}=$ $h q_{i+1} d x-h p_{i+1} d y$.

Se $\gamma \geq v_{i+1}$, então

$$
2 v\left(h p_{i+1}\right)-2 v_{0}+v_{1}=2 \gamma+2 v\left(p_{i+1}\right)-2 v_{0}+v_{1}>2 \gamma-v_{0} \geq \gamma+v_{i+1}-v_{0} .
$$

Assim pelo Lema 4.1, existe $c \in K^{*}$ tal que a mudança de coordenadas

$$
\begin{aligned}
& t_{1}=t\left(1+\frac{\operatorname{ch} p_{i+1}}{t^{v_{0}}}\right)^{\frac{1}{v_{0}}} \\
& y_{1}=y+c h q_{i+1},
\end{aligned}
$$


permite eliminar o termo de ordem $\gamma+v_{i+1}-v_{0}$ sem que alteremos os termos anteriores.

Se $\gamma<v_{i+1}$, temos que $\gamma=\sum_{j=0}^{i} \alpha_{j} v_{j}$, logo divisível por $e_{i}$.

Usando que $F=\left\{h_{0}, h_{1}, \ldots, h_{g}\right\}$ é uma Base Standard Mínima para $\mathcal{O}$, podemos escrever $h=\sum_{\alpha \in A} c_{\alpha} \prod_{j=0}^{g} h_{j}^{\alpha_{j}} \operatorname{com} \alpha=\left(\alpha_{0}, \ldots, \alpha_{g}\right) \in \mathbb{N}^{g+1}$ e $v\left(\prod_{j=0}^{g} h_{j}^{\alpha_{j}}\right) \neq v\left(\prod_{j=0}^{g} h_{j}^{\delta_{j}}\right)$ sempre que $\alpha \neq \delta$ e $c_{\alpha} c_{\delta} \neq 0$.

Ao efetuarmos a mudança

$$
\begin{aligned}
& t_{1}=t\left(1+\frac{\operatorname{chp}_{i+1}}{t_{0}}\right)^{\frac{1}{v_{0}}} \\
& y_{1}=y+\operatorname{ch} q_{i+1},
\end{aligned}
$$

podemos eliminar o termo de ordem $\gamma+v_{i+1}-v_{0}$ escolhendo $c \in K$ convenientemente. No entanto, podemos ter alterado os termos de ordem inferior à $\gamma+v_{i+1}-v_{0}$ em $y_{1}$. Observe que as parcelas $c_{\alpha} \prod_{j=0}^{g} h_{j}^{\alpha_{j}}$ de $h$ que possuem ordem maior ou igual a $v_{i+1}$, e pela análise do caso anterior, não são responsáveis por essa alteração. Assim, sem perda de generalidade, podemos assumir que $h=\sum_{\alpha \in A} c_{\alpha} \prod_{j=0}^{i} h_{j}^{\alpha_{j}}$, ou seja, todas as parcelas $c_{\alpha} \prod_{j=0}^{g} h_{j}^{\boldsymbol{\alpha}_{j}}$ de $h$ possuem ordem em $\left\langle v_{0}, \ldots, v_{i}\right\rangle$. $\frac{v_{0}}{e_{i}}+1$.

Seja $\Gamma_{i}=\left\langle\frac{v_{0}}{e_{i}}, \ldots, \frac{v_{i}}{e_{i}}\right\rangle$, cujo condutor sabemos ser $\mu_{i}=\sum_{j=1}^{i}\left(n_{j}-1\right) \frac{v_{j}}{e_{i}}-$

A mudança de coordenadas acima ıos dá

$$
\left\{\begin{array}{l}
x_{1}=t_{1}^{v_{0}} \\
y_{1}=t_{1}^{v_{1}}+\sum_{l>v_{1}} a_{l} t^{l}+\frac{c\left(h q_{i+1} d x-h p_{i+1} d y\right)}{d x}-\sum_{l \geq v_{1}} a_{l} t^{l}\left(\sum_{j=2}^{\infty}\left(\begin{array}{c}
\frac{l}{v_{0}} \\
j
\end{array}\right)\left(\frac{c\left(h p_{i+1}\right)}{t^{v_{0}}}\right)^{j}\right) .
\end{array}\right.
$$

Veja que

$$
v\left(t^{l}\left(\frac{h p_{i+1}}{x}\right)^{j}\right)=l+j \gamma+j v\left(p_{i+1}\right)-j v_{0}
$$

Como $l \geq v_{1}, \gamma \geq 0$ e pela proposiçño anterior $v\left(p_{i+1}\right)=\sum_{k=1}^{i}\left(n_{k}-1\right) v_{k}$, temos que.

$$
\begin{aligned}
v\left(t^{l}\left(\frac{h p_{i+1}}{x}\right)^{j}\right) & \geq v_{1}+j \sum_{k=1}^{i}\left(n_{k}-1\right) v_{k}-j v_{0}= \\
& =v_{1}+j\left(\sum_{k=1}^{i}\left(n_{k}-1\right) v_{k}-v_{0}\right)> \\
& >v_{1}+e_{i} \mu_{i}-e_{i}>e_{i} \mu_{i} .
\end{aligned}
$$


Além disso, se $l \geq \beta_{i+1}$, onde $\beta_{0}<\beta_{1}<\cdots<\beta_{g}$ é a sequiência característica de $C$, e lembrando que $v_{i+1}=\sum_{k=1}^{i}\left(n_{k}-1\right) v_{k}+\beta_{i+1}$ temos que

$$
\begin{aligned}
& \dot{v}\left(t^{i}\left(\frac{h p_{i+1}}{x}\right)^{j}\right)-\geq \beta_{i+1}+j \gamma+j \sum_{k=1}^{i}\left(n_{k}-1\right) v_{k}-j v_{0}= \\
& \quad=(j-1) \sum_{k=1}^{i}\left(n_{k}-1\right) v_{k}-j v_{0}+v_{i+1}+j \gamma>\gamma+v_{i+1}-v_{0} .
\end{aligned}
$$

Uma vez que $e_{i} \mid l$ para todo $l<\beta_{i+1}$, a ordem de todos os termos introduzidos antes de $t^{\gamma+v_{i+1}-v_{0}}$ são divisíveis por $e_{i}$ e são maiores que $e_{i} \mu_{i}$, ou seja, existem $\alpha_{0}, \ldots, \alpha_{i} \in \mathbb{N}$ tais que $\prod_{j=0}^{i} h_{j}^{\alpha_{j}}$ assume a ordem destes termos. Assim, podemos eliminá-los por meio de uma mudança de coordenadas da forma

$$
\begin{aligned}
& t_{2}=t_{1} \\
& y_{2}=y_{1}-b \prod_{j=0}^{i} h_{j}^{\alpha_{j}},
\end{aligned}
$$

para algum $b \in K$ conveniente.

Como. $e_{i} \mid \gamma$ segue que $e_{i} \not\left(\gamma+v_{i+1}-v_{0}\right)$. Portanto, basta garantirmos que os termos, de ordem inferior a $\gamma+v_{i+1}-v_{0}$, introduzidos pela mundança acima, possuem ordem divisível por $e_{i}$ e superiores a $e_{i} \mu_{i}$, seguindo assim 0 resultado, pois poderemos repetir o mesmo procedimento eliminando-os da parametrização.

Pelos argumentos utilizados na prova da Proposição 2.1, segue que o termo de menor ordem $\delta$ em $\prod_{j=0}^{i} h_{j}^{\alpha_{j}}$ que não é divisível por $e_{i}$ é obtido como produto do termo de menor ordem cujo expoente não ś divisível por $e_{i}$ em um fator $h_{m}$, que sabemos ser $\sum_{k=1}^{m-1}\left(n_{k}-1\right) v_{k}+\beta_{i+1}$, pelos termos líderes dos demais $h_{j}$. Assim, como $v\left(p_{i+1}\right)+\beta_{i+1}=v_{i+1}$ e $v\left(\sum_{j=0}^{i} h_{j}^{\alpha_{j}}\right) \geq$ $v_{1}+2 \gamma+.2 v\left(p_{i+1}\right)-2 v_{0}$, pois este último número é a menor ordem possível de um termo introduzido na mudança de coordenadas (4.5), temos que $\delta$ é igual a

$$
\begin{aligned}
\min _{0 \leq m \leq i}\left\{\sum_{j=0, j \neq m}^{i} \alpha_{j} v_{j}+\left(\alpha_{m}-1\right) v_{m}+\sum_{k=1}^{m-1}\left(n_{k}-1\right) v_{k}+\beta_{i+1}\right\}= \\
\quad=\min _{0 \leq m \leq i}\left\{\sum_{j=0}^{i} \alpha_{j} v_{j}-\beta_{m}+\beta_{i+1}\right\}= \\
=\sum_{j=0}^{i} \alpha_{j} v_{j}-\beta_{i}+\beta_{i+1} \geq v_{1}+2 \gamma+2 v\left(p_{i+1}\right)-2 v_{0}-\beta_{i}+\beta_{i+1}= \\
=v_{1}+2 \gamma+v\left(p_{i+1}\right)-2 v_{0}-\beta_{i}+v_{i+1} \geq \\
\geq v_{1}+\gamma+\sum_{k=1}^{i} v_{k}-\beta_{i}-v_{0}+\gamma+v_{i+1}-v_{0}>\gamma+v_{i+1}-v_{0} .
\end{aligned}
$$


Portanto, o resultado segue.

A proposição anterior, nos diz que todas as classes de equivalência de curvas algebróides irredutíveis planas com semigrupo fixo $\Gamma=\left\langle v_{0}, v_{1}, \ldots, v_{g}\right\rangle$ podem ser representadas por uma parametrização da forma

$$
\left\{\begin{array}{l}
x=t^{v_{0}} \\
y=t^{v_{1}}+\sum_{l \in L} a_{l} t^{l},
\end{array}\right.
$$

oude $a_{l} \in K$ e $L$ é o conjunto das lacunas de $\Gamma$ que são maiores que $v_{1}$ e não são da forma $\gamma+v_{i}-v_{0} \operatorname{com} \gamma \in \Gamma$ e $i=0, \ldots, g$.

Zariski, em [Z2], mostra que se $\lambda$ é o invariante de Zariski de um ramo $C$, então existe uma mudança de coordenadas, de modo a podermos eliminar todos os termos de ordem $\lambda+\gamma \operatorname{com} \gamma \in\left\langle v_{0}, v_{1}\right\rangle \backslash\{0\}$, sem que alteremos os termos de ordem inferior.

Se $\Omega_{1} \in \mathcal{O} d \mathcal{O}$ é uma DNE de menor ordem, então sabemos que $v\left(\Omega_{1}\right)+1=$ $\lambda+v_{0}$. Deste modo, o resultado de Zariski, é um caso particular da proposição abaixo.

Proposição 4.3 Seja C uma curva algebróide irredutível plana com semigrupo $\Gamma=\left\langle v_{0}, v_{1}, \ldots, v_{g}\right\rangle$, dada por

$$
\left\{\begin{array}{l}
x=t^{v_{0}} \\
y=t^{v_{1}}+\sum_{l \in L} a_{l} t^{l},
\end{array}\right.
$$

com. $L=\left\{l \in \mathbb{N} \backslash \cup_{i=0}^{g}\left(\Gamma+v_{i}-v_{0}\right) ; l>v_{1}\right\}$. Se $\Omega_{1} \in \mathcal{O} d \mathcal{O}$ é uma DNE de menor ordem, então existe uma mudança de coordenadas de modo que $C$ é equivalente a

$$
\left\{\begin{array}{l}
x=t^{v_{0}} \\
y=t^{v_{1}}+\sum_{l \in L \backslash\left(\Gamma^{*}+v\left(\Omega_{1}\right)+I-v_{0}\right)} a_{l}^{\prime} t^{l},
\end{array}\right.
$$

Dem.: Seja $\Omega_{1} \in \mathcal{O} d \mathcal{O}$ uma DNE de menor ordem. Pelo que vimos logo após a Observação 3.2 , temos que $v\left(\Omega_{1}\right)+1=\lambda+v_{0}$, onde $\lambda$ é o iuvariante de Zariski de $C$. Podemos considerar $C$ dada por uma parametrização

$$
\left\{\begin{array}{l}
x=t^{v_{0}} \\
y=t^{v_{1}}+a_{\lambda} t^{\lambda}+\sum_{l \in L} a_{l} t^{l} .
\end{array}\right.
$$


Inicialmente, mostremos como eliminar as lacunas $l$, tais que $l-\lambda \in \Gamma^{*}$ e $l-\lambda<v_{2}$, ou seja, $l=\lambda+\gamma$, com $\gamma=\alpha v_{0}+\beta v_{1}>0$.

Considere $p=x\left(1+c x^{\alpha} y^{\beta}\right)^{v_{0}}-x=\sum_{i=1}^{v_{0}}\left(\begin{array}{c}v_{0} \\ i\end{array}\right) c^{i} x^{i \alpha+1} y^{i \beta}$, com $c \in K^{*} \mathrm{a}$ ser caracterizado posteriormente.

Tomemos a mudança de parâmetros

$$
t_{1}=t\left(1+\frac{p}{t^{v_{0}}}\right)^{\frac{1}{v_{0}}}=t\left(1+c x^{\alpha} y^{\beta}\right)=t+c t x^{\alpha} y^{\beta}
$$

Desse modo, temos

$$
y\left(t_{1}\right)=\left(t+c t x^{\alpha} y^{\beta}\right)^{v_{1}}+\sum_{\lambda \leq j<l} a_{j}\left(t+c t x^{\alpha} y^{\beta}\right)^{j}+a_{l} t^{l}+\begin{aligned}
& \text { termos de } \\
& \text { ordem }>l
\end{aligned} .
$$

Una vez que, $v\left(t^{i} x^{k \alpha} y^{k \beta}\right)>l$ sempre que $i>\lambda$ ou $i=\lambda$ e $k>1$ e que $\left(t+c t x^{\alpha} y^{\beta}\right)^{i}=t^{i}+\sum_{k=1}^{i}\left(\begin{array}{l}i \\ k\end{array}\right) c^{k} t^{i} x^{k \alpha} y^{k \beta}$, podemos escrever

$$
\begin{array}{r}
y\left(t_{1}\right)=t^{v_{1}}+\sum_{i=1}^{v_{1}}\left(\begin{array}{c}
v_{1} \\
i
\end{array}\right) c^{i} t^{v_{1}} x^{i \alpha} y^{i \beta}+a_{\lambda} t^{\lambda}+\sum_{\lambda<j<l} a_{j} t^{j}+ \\
+\left(a_{l}+\lambda c a_{\lambda}\right) t^{l}+\begin{array}{c}
\text { termos } \\
\text { de ordem } \\
>l
\end{array}
\end{array}
$$

Note que

$$
t^{v_{1}} x^{i \alpha} y^{i \beta}=t^{v_{1}+i \alpha v_{0}+i \beta v_{1}}+i \beta a_{\lambda} t^{\lambda+i \alpha v_{0}+i \beta v_{1}}+\cdots
$$

ou seja,

$$
y\left(t_{1}\right)=t^{v_{1}}+\sum_{i=1}^{v_{1}}\left(\begin{array}{c}
v_{1} \\
i
\end{array}\right) c^{i} t^{v_{1}+i \alpha v_{0}+i \beta v_{1}}+a_{\lambda} t^{\lambda}+\sum_{\lambda<j<l} a_{j} t^{j}+\left(a_{l}+\left(\lambda+v_{1} \beta\right) c a_{\lambda}\right) t^{l}+\cdots
$$

Agora observe que

$$
x^{i \alpha} y^{i \beta}=t^{v_{1}+i \alpha v_{0}+i \beta v_{1}}+(i \beta+1) a_{\lambda} t^{\lambda+i \alpha v_{0}+i \beta v_{1}}+\cdots .
$$

Assim,

$$
y\left(t_{1}\right)-\sum_{i=1}^{v_{1}}\left(\begin{array}{c}
v_{1} \\
i
\end{array}\right) c^{i} x^{i \alpha} y^{i \beta+1}=t^{v_{1}}+a_{\lambda} t^{\lambda}+\sum_{\lambda<j<l} a_{j} t^{j}+\left(a_{l}+\left(\lambda-v_{1}\right) c a_{\lambda}\right) t^{l}+\cdots,
$$


como $y=t^{v_{1}}+a_{\lambda} t^{\lambda}+\sum_{\lambda<j<l} a_{j} t^{j}+a_{i} t^{l}+\cdots$ e $y\left(t_{1}\right)=t_{1}^{v_{1}}+a_{\lambda} t_{1}^{\lambda}+\sum_{\lambda<j<l} a_{j} t_{1}^{j}+$ $a_{l} t^{l}+$ termos de ordem $>l$, temos que,

$$
y+\sum_{i=1}^{v_{1}}\left(\begin{array}{c}
v_{1} \\
i
\end{array}\right) c^{i} x^{i \alpha} y^{i \beta+1}=t_{1}^{v_{1}}+a_{\lambda} t_{1}^{\lambda}+\sum_{\lambda<j<l} a_{j} t_{1}^{j}-\left(a_{l}+\left(\lambda-v_{1}\right) c a_{\lambda}\right) t^{l}+\cdots
$$

Desse modo, tomando $c=-\frac{a_{l}}{\left(\lambda-v_{1}\right) a_{\lambda}}$ e a mudança de coordenadas

$$
\begin{aligned}
& t_{1}=t\left(1+\frac{p}{t^{v_{0}}}\right)^{\frac{1}{v_{0}}}=t\left(1+c x^{\alpha} y^{\beta}\right) \\
& y_{1}=y+\sum_{i=1}^{v_{1}}\left(\begin{array}{c}
v_{1} \\
i
\end{array}\right) c^{i} x^{i \alpha} y^{i \beta+1}
\end{aligned}
$$

a curva

$$
\left\{\begin{array}{l}
x_{1}=t_{1}^{v_{0}} \\
y_{1}=t_{1}^{v_{1}}+a_{\lambda} t_{1}^{\lambda}+\sum_{\lambda<j<l} a_{j} t_{1}^{j}+\sum_{j>l} a_{j}^{\prime} t_{1}^{j}
\end{array}\right.
$$

é equivalente a curva $C$.

Consideremos agora o caso em que $l=\lambda+\gamma$, com $\gamma \in \Gamma \backslash\left\langle v_{0}, v_{1}\right\rangle$, ou seja,

$$
\gamma=l-\lambda \geq v_{2}=\left(n_{1}-1\right) v_{1}+\beta_{2} \geq\left(n_{1}-1\right) v_{1}+\lambda>\lambda-v_{1} .
$$

Tomemos $p=\operatorname{chx}$ e $q=\frac{v_{1}}{v_{0}}$ chy, onde $c$ é uma constaute a ser determinada e $h \in \mathcal{O}$ tal que $v(h)=\gamma=l-\lambda$. A mudança de coordenadas

$$
\begin{aligned}
& t_{1}=t\left(1+\frac{p}{t^{v_{0}}}\right)^{\frac{1}{v_{0}}} \\
& y_{1}=y+q,
\end{aligned}
$$

110s fornece (Vide Exemplo 3.2)

$$
\left\{\begin{array}{l}
x_{1}=t_{1}^{v_{0}} \\
y_{1}=t_{1}^{v_{1}}+a_{\lambda} t_{1}^{\lambda}+\sum_{j>\lambda} a_{i j} t_{1}^{j}+\frac{q d x-p d y}{d x}-\sum_{j \geq v_{1}} a_{j} t^{j}\left(\sum_{k=2}^{\infty}\left(\begin{array}{c}
j \\
v_{0} \\
k
\end{array}\right)\left(\frac{p}{t^{v_{0}}}\right)^{k}\right) .
\end{array}\right.
$$

Como

$$
\begin{aligned}
v\left(\frac{q d x-p d y}{d x}\right) & =v\left(\operatorname{ch} \frac{\Omega_{1}}{d x}\right)= \\
& =\gamma+\lambda<2 \gamma+v_{1}=v\left(\sum_{j \geq v_{1}} a_{j} t^{j}\left(\sum_{k=2}^{\infty}\left(\begin{array}{c}
\frac{j}{v_{0}} \\
k
\end{array}\right)\left(\frac{p}{t^{v_{0}}}\right)^{k}\right)\right),
\end{aligned}
$$

podemos escolher $c \in K^{*}$, de modo que

$$
\frac{q d x-p d y}{d x}-\sum_{j \geq v_{1}} a_{j} t^{j}\left(\sum_{k=2}^{\infty}\left(\begin{array}{c}
\frac{j}{v_{0}} \\
k
\end{array}\right)\left(\frac{p}{t^{v_{0}}}\right)^{k}\right)=-a_{l} t^{l}+\cdots,
$$


como $t^{l}=t_{1}^{l}+\cdots$, obtemos uma curva equivalente à curva $C$ dada por uma parametrização da forma .

$$
\left\{\begin{array}{l}
x_{1}=t_{1}^{v_{0}} \\
y_{1}=t_{1}^{v_{1}}+a_{\lambda} t_{1}^{\lambda}+\sum_{\lambda<j<l} a_{j} t_{1}^{j}+\sum_{j>l} a_{j}^{\prime} t_{1}^{j} .
\end{array}\right.
$$

Mais do que a garantia da eliminação de determinados termos em uma parametrização de um ramo, as duas últimas proposições nos dão explicitamente as mudanças de coordenadas que devemos realizar para eliminar os termos em questão.

Observando que $v\left(d h_{i}\right)-v\left(d h_{0}\right)=v_{i}-v_{0}$, podemos unificar as duas últimas proposições, no segıinte teorema.

Teorema 4.1 Seja $C$ uma curva algebróide irredutível plana com semigrupo de valores $\Gamma=\left\langle v_{0}, v_{1}, \ldots, v_{g}\right\rangle$, dada por

$$
\left\{\begin{array}{l}
x=t^{v_{0}} \\
y=t^{v_{1}}+\sum_{j>v_{1}} a_{j} t^{j}
\end{array}\right.
$$

Seja $F=\left\{h_{0}, h_{1}, \ldots, h_{g}\right\}, \operatorname{com} v\left(h_{i}\right)=v_{i}$ para $i=0, \ldots, g$ e $\Omega_{1} \in \mathcal{O} d \mathcal{O}$ uma DNE de menor ordem, então $C$ é equivalente a

$$
\left\{\begin{array}{l}
x=t^{v_{0}} \\
y=t^{v_{1}}+\sum_{\substack{j>v_{1} \\
j \in \mathbb{N} \backslash L}} a_{j}^{\prime} t^{j}
\end{array}\right.
$$

onde $L=\left(\Gamma^{*}+v\left(\Omega_{1}\right)-v\left(d h_{0}\right)\right) \cup_{i=0}^{g}\left(\Gamma+v\left(d h_{i}\right)-v\left(d h_{0}\right)\right)$.

Exemplo 4.1 Seja $\Gamma=\left\langle v_{0}, v_{1}, \ldots, v_{g}\right\rangle$, com $v_{0}=4$ o semigrupo de uma curva algebróide irredutível plana $C$.

De acordo com a Observação 3.2, as lacunas $l$ de $\Gamma$ maiores que $v_{\mathrm{I}}$ são da forma

$$
\begin{aligned}
& v_{2}-j v_{0} \operatorname{com} 1 \leq j \leq\left[\frac{v_{2}-v_{1}}{v_{0}}\right] \\
& v_{2}+v_{1}-j v_{0} \operatorname{com} 1 \leq j \leq\left[\frac{v_{2}}{v_{0}}\right] .
\end{aligned}
$$


Como $e_{1}=M D C\left(v_{0}, v_{1}\right) \backslash v_{2}, m_{1}=\frac{v_{1}}{e_{1}}$ e $\beta_{2}=v_{2}+v_{1}-m_{1} v_{0}$ é o men.or expoente na parametrização de $C$ que não é divisivel por $e_{1}$, seque qus. $\beta_{2}$ é $o$ invariante de Zariski de $C$.

Note que as lacunas da forma $v_{2}+v_{1}-j v_{0}$, maiores que $\beta_{2}=\lambda$, podem ser expressas como

$$
v_{2}+v_{1}-m_{\cdot 1} v_{0}+\alpha v_{0}=\alpha v_{0}+v\left(\Omega_{1}\right)-v\left(d h_{0}\right),
$$

$\operatorname{com} \alpha>0$.

Portanto, pelo teorema anterior, tais lacunas podem ser eliminadas, bem como a lacuna $v_{2}-v_{0}$. Desse modo, basta considerarmos na parametrização de $C$ as lacunas da forma $v_{2}-j v_{0}$, com $2 \leq j \leq\left[\frac{v_{2}-v_{1}}{v_{0}}\right]$, que são maiores que. $\beta_{2}$, i.e., aquelas para as quais $2 \leq j \leq\left[\frac{v_{1}}{v_{0}}\right]$.

Uma vez que através de uma mudança de coordenadas da forma

$$
\begin{aligned}
& t_{1}=c t \\
& y_{1}=c^{v_{1}} y
\end{aligned}
$$

com, $c \in K^{*}$ conveniente, podemos tornar o coeficiente de $t^{\beta_{2}}$ igual a 1 , temos que todo ramo, com semigrupo $\Gamma=\left\langle 4, v_{1}, v_{2}\right\rangle$ é equivalente a um ramo do tipo

$$
\left\{\begin{array}{l}
x=t^{4} \\
y=t^{v_{1}}+t^{v_{2}+v_{1}-4 m_{1}}+\sum_{j=2}^{\left[\frac{v_{1}}{4}\right]} a_{j}^{\prime} t^{v_{2}-4}\left(\left[\frac{v_{1}}{4}\right]+2-j\right) .
\end{array}\right.
$$

Aplicando o Algoritmo 2.2, temos que $B=\left\{d x, d y, d h_{2}, \Omega_{1}\right\}$, com. $h_{2} F$ $y^{2}-x^{m_{1}}$ e $\Omega_{1}=x d y-\frac{v_{1}}{4} y d x$ é uma Base Standard Mínima para $\mathcal{O} d \mathcal{O}$. De fato, partindo de $\left\{d x, d y, d h_{2}\right\}$, a única DNE obtida no primeiro passo do algoritmo é $\Omega_{1}$ e a lacuna limitante pode ser considerada $l=v_{2}-4$. Uma simples análise, indica que a ordem da altura de todos os $S$-processos envolvendo $\Omega_{1}$ superam $l$, indicando o fim do algoritmo.

Portanto, podemos reescrever a parametrização acima como

$$
\left\{\begin{array}{l}
x=t^{4} \\
y=t^{v_{1}}+t^{\beta_{2}}+\sum_{\substack{l>\beta_{2} \\
l \notin v\left(\frac{\mathcal{O} d O}{d x}\right)}}^{i} a_{l}^{\prime} t^{l} .
\end{array}\right.
$$




\section{Capítulo 5}

\section{Ramos de Gênero 1}

Neste capítulo faremos o estudo sistemático das curvas algebróides irredutíveis planas de gênero 1 , isto é, daquelas que admitem semigrupo as-, sociado da forma $\Gamma=\left\langle v_{0}, v_{1}\right\rangle$.

\subsection{Base Standard e Lacunas Especiais}

Seja $C$ um ramo com gênero 1, i.e.,

$$
C:\left\{\begin{array}{l}
x=t^{v_{0}} \\
y=t^{v_{1}}+\sum_{i>v_{1}} a_{i} t^{i}
\end{array}\right.
$$

$\operatorname{com} a_{i} \in K$ e $\operatorname{MDC}\left(v_{0}, v_{1}\right)=1$.

O semigrupo de valores associado à curva $C$ é $\Gamma=\left\langle v_{0}, v_{1}\right\rangle$, cujo condutor é $\mu=\left(v_{0}-1\right)\left(v_{1}-1\right)$. Obviamente, $F=\{x, y\}$ é uma Base Standard Mínima para o anel local $\mathcal{O}$ de $C$.

Pela Observação 3.2 , vemos que as lacunas de $\Gamma$ maiores que $v_{1}$ são da forma $l=i v_{1}-j v_{0}$ com $i=2, \ldots, v_{0}-1$ e $j=1, \ldots,\left[\frac{(i-1) v_{1}}{v_{0}}\right]$.

Assim, pela Proposição 4.2 podemos assumir que $C$ seja dada por uma parametrização da forma

$$
C:\left\{\begin{array}{l}
x=t^{v_{0}} \\
y=t^{v_{1}}+\sum_{l \in L} a_{l} t^{l}
\end{array}\right.
$$

com $a_{l} \in K$ e $L=\left\{l=i v_{1}-j v_{0} ; 2 \leq i \leq v_{0}-1\right.$ e $\left.2 \leq j \leq\left[\frac{(i-1) v_{1}}{v_{0}}\right]\right\}$. 
Seja $\Lambda=\{v(\Omega)+, 1 ; \Omega \in \mathcal{O} d \mathcal{O}\}$. Como $\left[\frac{\left(v_{0}-2\right) v_{1}}{v_{0}}\right]<v_{1}$, tomando $\sigma=v_{1}$, o diagrama de lacunas de $C$ (veja Definição 3.3) pode ser obtido através da restrição à $\Lambda \backslash \Gamma$ da aplicação

$$
\varrho:{ }_{l=i v_{1}-j v_{0}} \quad \longrightarrow \quad \begin{gathered}
\mathbb{N}^{2} \\
\left(v_{1}-j, i\right) .
\end{gathered}
$$

Desse modo, o diagrama de lacumas de $C$ estará contido no retângulo $\left[0, v_{1}-1\right] \times\left[0, v_{0}-1\right] \subset \mathbb{N}^{2}$.

A ordenação dos elementos de $\varrho(\Lambda)$, em particular dos pontos no diagrama de lacunas, pode ser feita, como vinos no Lema 3.1, através do coeficiente. linear da reta de inclinação $-\frac{v_{0}}{v_{1}}$ que passa por um ponto de $\varrho(\Lambda)$, ou seja,

$$
\left(x_{0}, y_{0}\right) \prec\left(x_{1}, y_{1}\right) \Leftrightarrow\left\{\begin{array}{l}
v_{1} y_{0}+v_{0} x_{0}<v_{1} y_{1}+v_{0} x_{1} \\
\text { ou } \\
v_{1} y_{0}+v_{0} x_{0}=v_{1} y_{1}+v_{0} x_{1} \text { e } x_{0}<x_{1}
\end{array}\right.
$$

Note que esta ordenação sugere pesos para as coordenadas dos pontos de $\mathbb{N}^{2}$. De fato, tomando $\rho=\left(v_{0}, v_{1}\right)$, a ordem acima é a ordem pesada com respeito ao peso $\rho$ e à ordem lexicográfica (Veja Seção 1.1).

Seja $f \in K[[X, Y]]$ uma equação cartesiana para $C$. Temos que.

$$
f(X, Y)=\prod_{j=0}^{v_{0}-1}\left(Y-\left(\xi^{j} X^{\frac{1}{v_{0}}}\right)^{v_{1}}-\sum_{i>v_{1}} a_{i}\left(\xi^{j} X^{\frac{1}{v_{0}}}\right)^{i}\right)=Y^{v_{0}}+\sum_{i=1}^{v_{0}} c_{i}(X) Y^{v_{0}-i}
$$

onde $\xi$ é uma raiz $v_{0}$-ésima primitiva da unidade e $c_{i}(X) \in K[[X]]$ com $\operatorname{ord}_{X}\left(c_{i}(X)\right) \geq \frac{i v_{1}}{v_{0}}$, para todo $i=1, \ldots, v_{0}$.

Seja $\mathcal{J}=\left\langle f_{X}, f_{Y}\right\rangle \mathcal{O}$ o ideal Jacobiano de $C$ e seja $\mathcal{I}=$ $\left\langle f, f_{X}, f_{Y}\right\rangle K[[X, Y]]$. Uma vez que

$$
\frac{K[[X, Y]]}{\mathcal{I}}=\frac{\mathcal{O}}{\mathcal{J}}
$$

podemos calcular o número $\tau$ de Tjurina de $C$ fixando uma ordem monomial em $K[[X, Y]]$ e computando uma Base Standard para o ideal $\mathcal{I}$ de $K[[X, Y]]$ (Veja [Bec1] e [Bec2]). Isto permite calcular $\sharp(\Lambda \backslash \Gamma)$, mas não permite exibir os elementos de $\Lambda \backslash \Gamma$.

Mostraremos que se a ordem monomial dada a $K[[X, Y]]$ for a ordem pesada acima descrita, então o conjunto $\Lambda \backslash \Gamma$ pode ser obtido facilmente. 
Para tanto, seja $B=\left\{f_{0}, \ldots, f_{s}\right\}$ a Base Standard Reduzida de $\mathcal{I}$, com respeito à ordem pesada definida anteriormente. Assim,

$$
f_{i}=X^{\alpha_{i}} Y^{\beta_{\imath}}+\sum_{j \in J} b_{j} X^{\alpha_{i j}} Y^{\beta_{i j}}
$$

com $b_{j} \in K^{*}, X^{\alpha_{k}} Y^{\beta_{k}} \chi X^{\alpha_{i j}} Y^{\beta_{i j}}$ para $i, k=0, \ldots, s$ e $j \in J$, e $X^{\alpha_{k}} Y^{\beta_{k}} \chi$ $X^{\alpha_{i}} Y^{\beta_{i}}$ se $k \neq i$.

Reordenando os elementos de $B$, podemos assumir sem perda de generalidade que $0=\alpha_{0}<\cdots<\alpha_{s}$ e $\beta_{0}>\cdots>\beta_{s}=0$ (Note que o fato de $\alpha_{0}=\beta_{s}=0$ segue da codimensão de $\mathcal{I}$ ser finita).

Considerando o par $\left(\alpha_{i}, \beta_{i}\right) \in \mathbb{N}^{2}$ para $i=0, \ldots, s$, vemos que a cada ponto de $\Delta=\mathbb{N}^{2} \backslash \bigcup_{i=0}^{s}\left\{\left(\alpha_{i}, \beta_{i}\right)+\mathbb{N}^{2}\right\}$ corresponde o monômio $X^{\alpha_{i}} Y^{\beta_{i}}$, cujas classes residuais formam uma base do espaço vetorial $\frac{K \| X, Y]]}{\mathcal{I}}$ sobre $K$.

Assim, o conjunto $\Delta \subseteq \mathbb{N}^{2}$ pode ser representado por

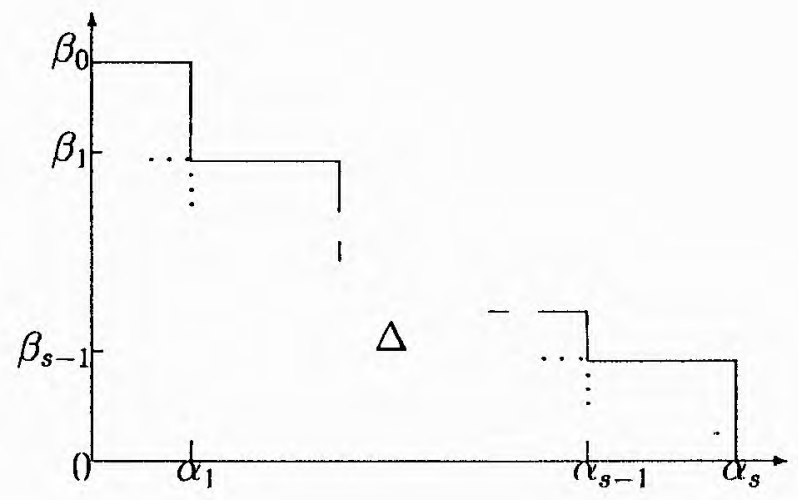

e $\tau=\operatorname{dim}_{K} \frac{K[X, Y]]}{\mathcal{I}}=\sum_{i=1}^{s} \alpha_{i}\left(\beta_{i-1}-\beta_{i}\right)$.

Observação 5.1 Uma vez que $B$ é a Base Standard Reduzida de $\mathcal{I}_{;}$; dado um elemento $f_{i}=X^{\alpha_{i}} Y^{\beta_{i}}+\sum_{j \in J} b_{j} X^{\alpha_{i j}} Y^{\beta_{i j}} \in B$, os coeficientes. $b_{j}$ podem ser não nulos, somente na região assinalada na figura abaixo. 


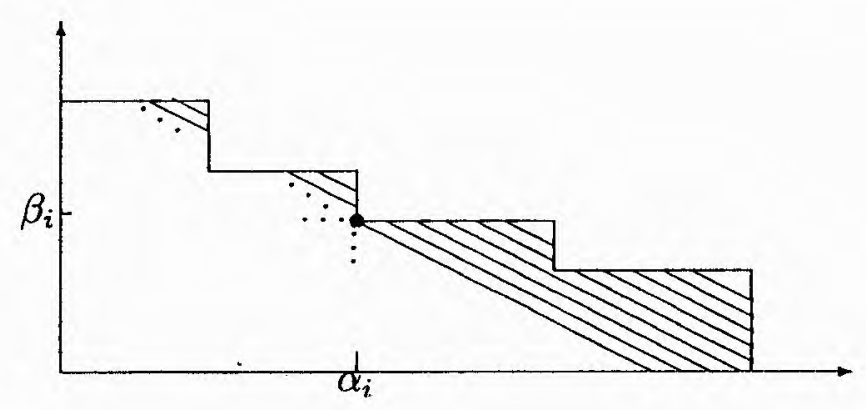

Vejamos mais detalhadamente a Base Standard Reduzida $B$ de $\mathcal{I}=$ $\left\langle f, f_{X}, f_{Y}\right\rangle$.

- Escrevamos $f(X, Y)=\sum_{k \geq v_{0} v_{i}} f_{k}$, onde cada $f_{k}$ é um polinômio de grau pesado $k$. Note que, se $i v_{0}+j v_{1}>v_{0} v_{1}$, então da expressão de $f$ em (5.1) temos que $f_{i v_{0}+j v_{1}}$ é um monômio da forma $a_{i j} X^{i} Y^{j}$. Assim,

$$
\begin{aligned}
& f(X ; Y)=Y^{v_{0}}-a X^{v_{1}}+\sum_{i v_{0}+j v_{1}>v_{0} v_{1}} a_{i j} X^{i} Y^{j} \\
& f_{X}(X, Y)=-a v_{1} X^{v_{1}-1}+\sum_{i v_{0}+j v_{1}>v_{0} v_{1}-v_{0}} b_{i j} X^{i} Y^{j} \\
& f_{Y}(X, Y)=v_{0} Y^{v_{0}-1}+\sum_{i v_{0}+j v_{1}>v_{0} v_{1}-v_{1}} c_{i j} X^{i} Y^{j}
\end{aligned}
$$

com $a_{,}, a_{i j}, b_{i j}$ e $c_{i j}$ em $K$.

Aplicando o algoritmo que nos dá a Base Standard Reduzida $B$ do ideal $\mathcal{I}$ (Veja [Beç1] e [Bec2]), vemos que $f$ não figura em $B$, uma vez que a potệncia líder de $f_{Y}$ divide a potência líder de $f$.

A saber, podemos tomar $B=\left\{f_{-1}, f_{0}, f_{1}, \ldots, f_{r}\right\}$ com

$$
\begin{aligned}
& f_{-1}=Y^{v_{0}-1}+\sum_{\substack{(i, j) \in \Delta \\
j v_{1}+i v_{0}>\left(v_{0}-1\right) v_{1}}} c_{i j}^{\prime} X^{i} Y^{j} \\
& f_{0}=X^{v_{1}-1}+\sum_{\substack{(i, j) \in \Delta \\
j v_{1}+i v_{0}>\left(v_{1}-1\right) v_{0}}} b_{i j}^{\prime} X^{i} Y^{j} \\
& f_{k}=X^{\alpha_{k}} Y^{\beta_{k}}+\sum_{\substack{(i, j) \in \Delta \\
j v_{1}+i v_{0}>\alpha_{k} v_{0}+\beta_{k} v_{1}}} d_{i j k} X^{i} Y^{j}
\end{aligned}
$$


$\operatorname{com} c_{i j}^{\prime}, b_{i j}^{\prime}, d_{i j k} \in K, k=1, \ldots, r \mathrm{e}$

$$
Y^{v_{0}-1} \prec X^{v_{1}-1} \prec X^{\alpha_{1}} Y^{\beta_{1}} \prec \cdots \prec X^{\alpha_{r}} Y^{\beta_{r}} .
$$

As séries $f_{-1}$ e $f_{0}$ são respectivamente as reduções completas módulo $B$ de $f_{Y}$ e $f_{X}$, que certamente estão presentes em $B$ pois os elementos obtidos pelo algoritmo de Buclıberger tềm potências líderes maiores que a altura dos $S$-processos $S\left(f, f_{X}\right), S\left(f, f_{Y}\right)$ e $S\left(f_{X}, f_{Y}\right)$.

Dessa forma, temos que $\operatorname{deg}_{\rho}\left(f_{k}\right)=v_{0} \alpha_{k}+v_{1} \beta_{k}>v_{0}\left(v_{1}-1\right)$, é portanto, $\alpha_{k} \geq v_{1}-1-\left[\frac{\beta_{k} v_{1}}{v_{0}}\right]$ para $k=1, \ldots, r$.

Como dois pontos de coordenadas inteiras em $\left[0, v_{1}-1\right] \times\left[0, v_{0}-1\right]$, nunca estão sobre uma mesma reta de inclinação $-\frac{v_{0}}{v_{1}}$, temos que a úuica potência com grau pesado $v_{0} \alpha_{k}+v_{1} \beta_{k}$ em $f_{k}$ é a potêucia líder, $\operatorname{assim} \operatorname{de} g_{\rho}\left(f_{k}\right)=$ $\alpha_{k} v_{0}+\beta_{k} v_{1}=v\left(f_{k}\right)=I\left(f, f_{k}\right), \operatorname{com} 0<\beta_{k}<v_{0}-1$ e $v_{1}-1-\left[\frac{\underline{\beta}_{k} v_{1}}{\eta_{0}}\right] \leq \alpha_{k}<$ $v_{1}-1 ; k=1, \ldots, r$, onde as cotas para $\beta_{k}$ e $\alpha_{k}$ decorrem do fato de $B$ ser a Base Standard Reduzida de $\mathcal{I}$.

Desse modo, se $\varphi$ é o monomorfismo definido em (2.1), então $\varphi(B) \subseteq$ $K[[t]]$ é uma Base Standard Mínima de $\mathcal{J}$ com respeito à ordem monomial de $K[[t]]$ dada pela valorização $v$ de $\overline{\mathcal{O}}$.

Portanto, podemos encontrar o conjunto das lacunas especiais $\Lambda \backslash \Gamma$ computando a Base Standard Reduzida $B$ para $\mathcal{I}=\left\langle f, f_{X}, f_{Y}\right\rangle \subseteq K[[X, Y]] \mathrm{com}$ respeito á ordem monomial pesada dada pelo peso $\rho=\left(v_{0}, v_{1}\right)$ e a ordem lexicográfica. Na verdade, basta que $B$ seja mínima. Para isso, extraímos de $B$ os elementos $f_{k}$ tais que $\operatorname{deg}_{\rho}\left(f_{k}\right)=v\left(f_{k}\right)=\beta_{k} v_{1}+\alpha_{k} v_{0} \operatorname{com} 0<\beta_{k}<v_{0}-1$ e $v_{1}-1-\left[\frac{\beta_{k} v_{1}}{v_{0}}\right] \leq \alpha_{k}<v_{1}-1$. Assim, usando o isomorfismo $\phi$, definido em (2.6), temos que $\phi\left(\varphi\left(f_{k}\right)\right)$ é a imagem pelo homomorfismo $\psi$ definido em (2.2), de uma DNEM. Consequientemente, pela Proposição 2.3

$$
\begin{aligned}
v\left(\phi\left(\varphi\left(f_{k}\right)\right)\right)+1 & =v\left(\varphi\left(f_{k}\right)\right)+1-\mu=v\left(f_{k}\right)+1-\mu= \\
& =\beta_{k} v_{1}+\alpha_{k} v_{0}-\left(v_{0}-1\right)\left(v_{1}-1\right)+1= \\
& =\left(\beta_{k}+1\right) v_{1}-\left(v_{1}-1-\alpha_{k}\right) v_{0}
\end{aligned}
$$

é a lacuna especial associada a uma DNEM. É claro que apartir destas lacuuas especiais obtemos facilmente $\Lambda \backslash \Gamma$.

Exemplo 5.1 Seja $f(X, Y)=Y^{4}-2 X^{5} Y^{2}-4 X^{7} Y-X^{9}+X^{10}$, cujo semigrupo de valores é $\Gamma=\langle 4,9\rangle$. Fixemos em $K[[X, Y]]$ a ordem pesada com. respeito ao peso $\rho=(4,9)$ e à ordem lexicográfica. 
Aplicando o algoritmo para obter uma Base Standard Mínima $B$ para $\mathcal{I}=\left\langle f, f_{X}, f_{Y}\right\rangle$, temos $B=\left\{f_{Y}, f_{X}, f_{1}, f_{2}\right\}$, onde

$$
\begin{gathered}
f_{1}(X, Y)=f-\frac{1}{4} Y f_{Y}-\frac{1}{9} X f_{X}=\frac{1}{9} X^{7} Y+\frac{1}{9} X^{5} Y^{2}-\frac{1}{9} X^{10} \\
f_{2}(X, Y)=-\frac{1}{81} Y f_{X}-X f_{1}=\frac{19}{9} X^{6} Y^{2}+\frac{10}{81} X^{4} Y^{3}+\frac{1}{9} X^{11}-\frac{10}{81} X^{9} Y .
\end{gathered}
$$

A escada de $\mathcal{I}$, o conjunto $\Delta$ e o diagrama de lacunas de $f$ podem ser dados em uma mesma figura, a saber

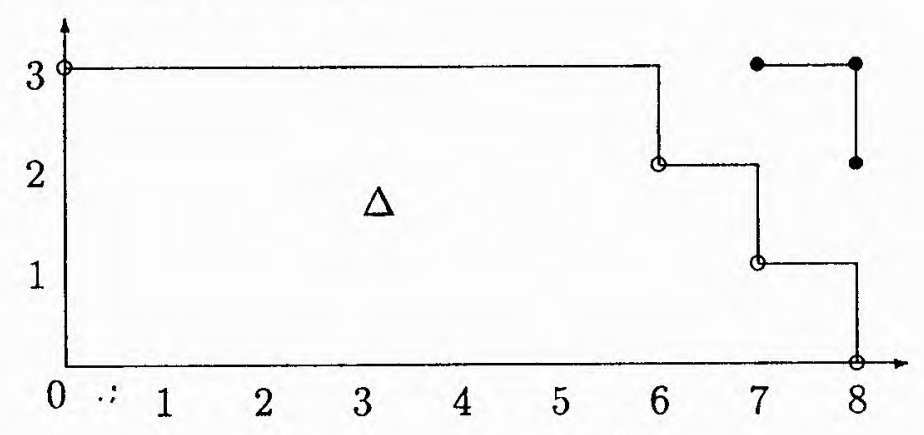

Note que se $v\left(f_{k}\right)=\beta_{k} v_{1}+\alpha_{k} v_{0}$ para $k=1,2$, então temos o ponto $\left(\alpha_{k}, \beta_{k}\right)$ na escada de $\mathcal{I}$. Como $v\left(\phi\left(\varphi\left(f_{k}\right)\right)\right)+1=\left(\beta_{k}+1\right) v_{1}-\left(v_{1}-1-\alpha_{k}\right) v_{0}$, teremos o ponto $\left(\alpha_{k}+1, \beta_{k}+1\right)$ no diagrama de lacunas.

Portanto, como $\mu=24$ e $\sharp(\Lambda \backslash \Gamma)=3$, a saber $\Lambda \backslash \Gamma=\{14,19,23\}$, temos $\tau=21$.

Observação 5.2 Note que em uma Base Standard Mínima de $\mathcal{I}=$ $\left\langle f, f_{X}, f_{Y}\right\rangle$ com respeito à ordem pesada dada pelo peso $\rho=\left(v_{0}, v_{1}\right)$ e pela ordem lexicográfica, temos elementos com termos líderes $Y^{v_{0}-1}$ e $X^{v_{1}-1} q u e$ correspondem aos pontos $\left(0, v_{0}-1\right)$ e $\left(v_{1}-1,0\right)$ na escada de $\mathcal{I}$ e que não contribuirão para o diagrama de lacunas. Desse modo, o conjunto $\Delta$ estará contido no retângulo $\left[0, v_{1}-1\left[\times\left[0, v_{0}-1\left[\right.\right.\right.\right.$, que contém $\mu=\left(v_{1}-1\right)\left(v_{0}-1\right)=$ $\operatorname{dim}_{K} \frac{K[[X, Y]]}{\left\langle f_{X}, f_{Y}\right\rangle}$ pontos.

- Uma vez que além dos pontos $\left(0, v_{0}-1\right)+\mathbb{N}^{2} e\left(v_{1}-1,0\right)+\mathbb{N}^{2}$ temos outros $\sharp(\Lambda \backslash \Gamma)$ que não contribuem para $\Delta$ e que estão no referido retângulo, obtemos assim, um outro modo de recuperar a relação

$$
\tau=\sharp \Delta=\mu-\sharp(\Lambda \backslash \Gamma) .
$$




\subsection{Ramos com Invariante de Zariski Fixo}

Como já observamos, o menor elemento de $\Lambda \backslash \Gamma$, se houver, é $\lambda+v_{0}$, onde $\lambda$ é o invariante de Zariski. A saber, $\lambda$ é a menor potência na parametrização de $y$ que nã̃o pertence à $\Gamma \cup\left\{\Gamma+v_{1}-v_{0}\right\}$. Dessa forma, podemoś estudar as

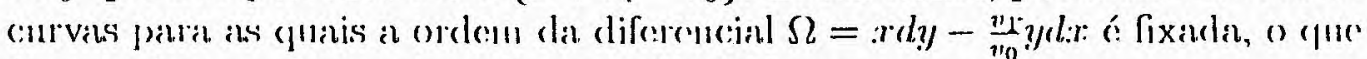
é equivalente a fixar o invariante de Zariski para a curva $C$.

Nesta seção, nos preocuparemos com o caso de curvas algebróides irredutíveis planas, com invariante de Zariski fixo, i.e., curvas que podem ser parametrizadas por

$$
C:\left\{\begin{array}{l}
x=t^{v_{0}} \\
y=t^{v_{1}}+t^{\lambda}+\sum_{i>\lambda} a_{i} t^{i}
\end{array}\right.
$$

$\operatorname{com} a_{i} \in K$.

A Proposição 3.3, aplicada às curvas de gênero 1 , permite reconhecer os $S$-processos essenciais para obter uma Base Standard Mínima para $\mathcal{O} d \mathcal{O}$, apartir de $\{d x, d y\}$.

Usando a relação entre uma Base Standard Mínima de $\mathcal{I}=\left\langle f, f_{X}, f_{Y}\right\rangle$ com respeito à ordem pesada dada pelo vetor peso $\rho=\left(v_{0}, v_{1}\right) \in \mathbb{N}^{2}$ e à ordem lexicográfica, e uma Base Standard Mínima para $\mathcal{O} d \mathcal{O}$, podemos recuperar alguns resultados conliecidos.

Em [D2], Delorme apresenta um algoritmo numérico que permite calcular o número de Tjurina e o conjunto $\Lambda \backslash \Gamma$ no caso genérico para uma classe de equisingularidade qualquer de gệnero 1 . Tal algoritmo é de cuulıo combinatório e muito específico para gênero 1.

Em $[B G M]$, Briançon, Granger e Maisonobe apresentam um algoritmo para obter a escada do ideal (Base Standard do ideal) $\mathcal{I}=\left\langle f, f_{X}, f_{Y}\right\rangle$ com respeito à ordem pesada dada pelo vetor $\rho$, onde $f(X, Y)=Y^{v_{0}}+a X^{v_{1}}+$ $\sum_{\alpha_{i} v_{0}+\beta_{i} v_{1}>v_{0} v_{1}} c_{i} X^{\alpha_{i}} Y^{\beta_{i}}$ é irredutível, $v_{0}$ e $v_{1}$ uão necessarianiente coprimos e os coeficientes $c_{i} \in K$ são genéricos. Note que, isto equivale a calcular o uúmero de Tjurina múnimo para todas as curvas cujo semigrupo é arbittário tendo apenas os dois primeiros geradores $v_{0}$ e $v_{1}$ fixados.

No caso em que $\operatorname{MDC}\left(v_{0}, v_{1}\right)=1$, i.e., ramos de gênero 1 , Briançou, Granger e Maiśonobe mostram como obter a fórmula que Delorme apresenta em [D2], e que nos dá o valor mínimo para $\tau$ para curvas com semigrupo $\Gamma=\left\langle v_{0}, v_{1}\right\rangle$. A saber,

$$
\tau=\left(v_{0}-1\right)\left(v_{1}-1\right)-\frac{\left(v_{1}-2\right)\left(v_{0}-2\right)}{4}+\frac{l_{0}}{4}-\frac{t_{1}}{2}\left(r_{1}+t_{2}-2\right)-\frac{1}{2},
$$


onde

$$
\frac{v_{1}}{v_{0}}=r_{1}+\frac{1}{r_{2}+\frac{1}{\vdots}}
$$

e os $l_{i}$ 's, bem como os $t_{i}$ 's, são dados pelas seguintes leis de recorrência decrescentes

$$
\begin{gathered}
l_{k}=0, \\
t_{k}=1, \\
l_{i-1}=l_{i}+t_{i} r_{i}, \\
t_{i-1}=\left\{\begin{array}{l}
0 \text { se } t_{i}=1 \text { e } l_{i-1} \text { é par } \\
1 \text { caso contrário. }
\end{array}\right.
\end{gathered}
$$

É relevante observamos que, apesar da fórmula acima nos fornecer $\sharp(\Lambda \backslash \Gamma)$, ela não dá nenhuma indicação sobre os valores das lacunas especiais.

Em [Pe2], Peraire, através de uma pequena modificação no passo inicial do algoritmo de Briançon, Granger e Maisonobe, obtém um algoritmo para determinar a escada de $\mathcal{I}=\left\langle f, f_{X}, f_{Y}\right\rangle$, onde $f$ é genérica, $C$ possui semigrupo $\Gamma=\left\langle v_{0}, v_{1}\right\rangle$ e o invàriante $\lambda$ de Zariski de $C$ é arbitrariamente fixado. É claro que neste caso $v_{0}>2$, pois caso contrário não haveria $\lambda$.

No que tange o cálculo do número $\tau$ de Tjurina mínimo, esse algoritıno é essencialmente o Algoritmo 2.2 quando este é aplicado à curva genérica. Observe quie nosso algoritmo, além de poder ser aplicado a qualquer curva e não necessariamente às curvas genéricas, fornece as lacunas especiais e uma Base Standard de $\mathcal{O} d \mathcal{O}$ em qualquer gênero.

Apresentamos abaixo o algoritmo dado por Delorme, em [D2], estendido por Briançon, Granger e Maisonobe, em [BGM] e modificado por Peraire, em [Pe2], interpretado no contexto da teoria aqui desenvolvida no caso em que $C$ é uma curva algebróide irredutível plana com semigrupo $\Gamma=\left\langle v_{0}, v_{1}\right\rangle$ e com invariante $\lambda^{*}$ de Zariski fixado.

Teorema 5.1 Seja

$$
C:\left\{\begin{array}{l}
x=t^{v_{0}} \\
y=t^{v_{1}}+t^{\lambda}+\sum_{i>\lambda} a_{i} t^{i}
\end{array}\right.
$$

onde $M D C\left(v_{0}, v_{1}\right)=1, \lambda=\alpha v_{1}-\beta v_{0}$,é o invariante de Zariski e os coeficientes $a_{i} \in K$ são considerados genéricos. 
Considerando a aplicação

$$
\varrho: \begin{array}{ccc}
\Lambda \backslash \Gamma & \longrightarrow & \mathbb{N}^{2} \\
i v_{1}-j v_{0} & \mapsto & \left(v_{1}-j, i\right),
\end{array}
$$

o conjunto das lacunas especiais de $C$ pode ser obtido através do seguinte algoritmo:

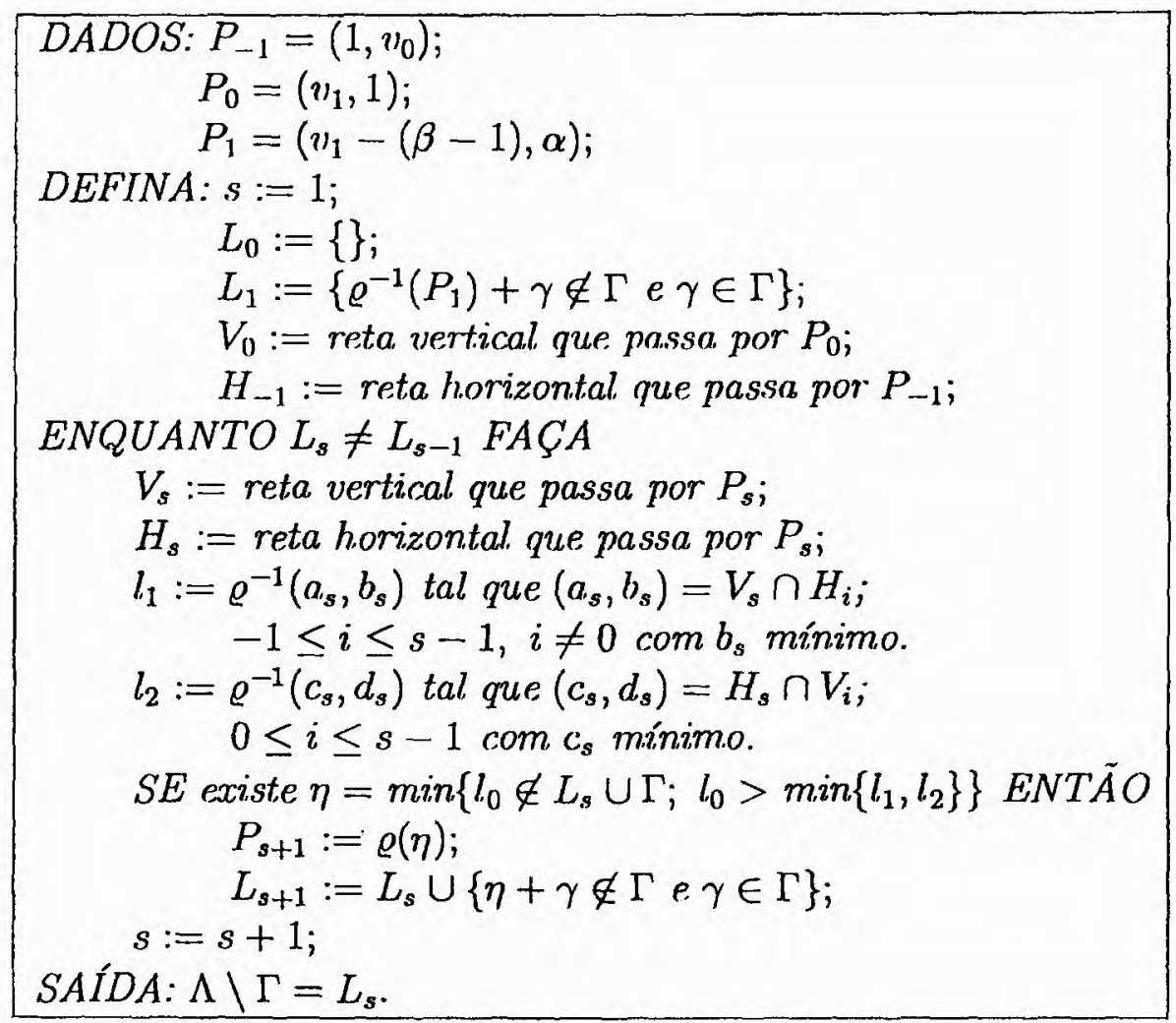

Dem.: Segundo o Algoritmo 2.2, iniciamos com $B=\{d x, d y\}$ e $F=\{x, y\}$.

O único $S$-processo a ser considerado 110 primeiro passo, que coincide com sua redução final módulo $(B, F)$ é $\Omega_{1}=x d y-\frac{v_{1}}{v_{0}} y d x$, cuja ordem é $\lambda+v_{0}-1$.

Desse modo, $P_{1}=\varrho\left(v\left(\Omega_{1}\right)+1\right)$ e $L_{1}=\left\{v\left(\Omega_{1}\right)+1+\gamma \notin \Gamma ; \gamma \in \Gamma\right\}$ é parte do collunto das lacunas especiais do $C$.

Note que os pontos $P_{-1}=\left(1, v_{0}\right)$ e $P_{0}=\left(v_{1}, 1\right)$ correspondem, por meio da associação $\varrho$, aos valores de $d x$ e $d y$ respectivamente.

Esses fatos, justificam os dados no ínicio do algoritmo, bem como a definição de $L_{1}$. 
Como $L_{0} \neq L_{1}$, definimos as retas $V_{1}$ e $H_{1}$. Segundo a Proposição 3.3 , o próximo $S$-processo a ser considerado será obtido apartir do menor valor $\varrho^{-1}\left(a_{1}, b_{1}\right)$ ou $\varrho^{-1}\left(c_{1}, d_{1}\right)$, onde $\left(a_{1}, b_{1}\right)$ é a interseção de $H_{1}$ com $V_{0}$, e $\left(c_{1}, d_{1}\right)$ é a interseção de $V_{1}$ com $H_{-1}$.

Se existe uma lacuna $l$ tal que $l \notin L_{1}$ e $l>\min \left\{\varrho^{-1}\left(a_{1}, b_{1}\right), \varrho^{-1}\left(c_{1}, d_{1}\right)\right\}$ tome a menor delas, então a generalidade dos coeficientes da parametrização de $C$, permite garantir que uma redução final do $S$-processo acima descrito, módulo $B=\left\{d x, d y, \Omega_{1}\right\}$ e $F$ será uma DNEM $\Omega_{2}$, com $v\left(\Omega_{2}\right)+1=l$. Neste caso, definimos $P_{2}=\varrho(l)$ e $L_{2}=L_{1} \cup\{l+\gamma \notin \Gamma ; \gamma \in \Gamma\}$ é um subconjunto de $\Lambda \backslash \Gamma$.

Se não houver tal lacuna $l$, então $B$ será fechado com respeito à formação de $S$-processos, indicando que $B$ é uma Base Standard Mínima para $\mathcal{O} \mathcal{O}$, e nesse caso $\Lambda \backslash \Gamma=L_{1}$.

Suponha agora que $k$ passos do algoritmo acima tenham sido realizados, i.e., tenhamos $B=\left\{d x, d y, \Omega_{1}, \ldots, \Omega_{k}\right\}$. Pela Proposição 3.3, basta analisar o $S$-processo envolvendo $\Omega_{k}$, obtido pelo menor valor $\varrho^{-1}\left(a_{k}, b_{k}\right)$ ou $\varrho^{-1}\left(c_{k}, d_{k}\right)$, onde $\left(a_{k}, b_{k}\right)$ é a interseção de $H_{k} \operatorname{com} V_{i}$ para $0 \leq i \leq k-1 \operatorname{com} b_{k}$ mínimo e $\left(c_{k}, d_{k}\right)$ é a interseção de $V_{k} \operatorname{com} H_{i}$ para $-1 \leq i \leq k-1, i \neq 1$ e $c_{k}$ mínimo.

Se houver uma lacuna $l \notin L_{k} \operatorname{com} l>\min \left\{\varrho^{-1}\left(a_{k}, b_{k}\right), \varrho^{-1}\left(c_{k}, d_{k}\right)\right\}$ tome a menor delas, então a generalidade dos coeficientes da parametrização garante. que haverá uma redução final deste $S$-processo módulo $(B, F)$, de modo a obtermos uma nova DNEM $\Omega_{k+1}$, com $v\left(\Omega_{k+1}\right)+1=l$. Neste caso, $L_{k+1}=$ $L_{k} \cup\{l+\gamma \notin \Gamma ; \gamma \in \Gamma\}$ é um subconjunto de $\Lambda \backslash \Gamma$ e o algoritmo prossegue $\operatorname{com} B=\left\{d x, d y, \Omega_{1}, \ldots, \Omega_{k+1}\right\}$, a saber, $P_{k+1}=\varrho\left(v\left(\Omega_{k+1}\right)+1\right)$.

Se não houver a lacuna $l$, acima descrita, o algoritmo finaliza com $\Lambda \backslash \Gamma=$ $L_{k}$.

Exemplo 5.2 Seja $\Gamma=\left\langle v_{0}, v_{1}\right\rangle=\langle 4,9\rangle$. Para o diagrama de lacunas, usaremos como referencial a lacuna $\mu-1=23$, representada pelo ponto localizado no canto superior direito do mesmo.

a) Considere $\lambda=11=3 v_{1}-4 v_{0}$. Aplicando o algoritmo anterior, temos $P_{-1}=(1,4), P_{0}=(9,1)$ e $P_{1}=(6,3)$ e $L_{1}=\{15,19,23\}$. 


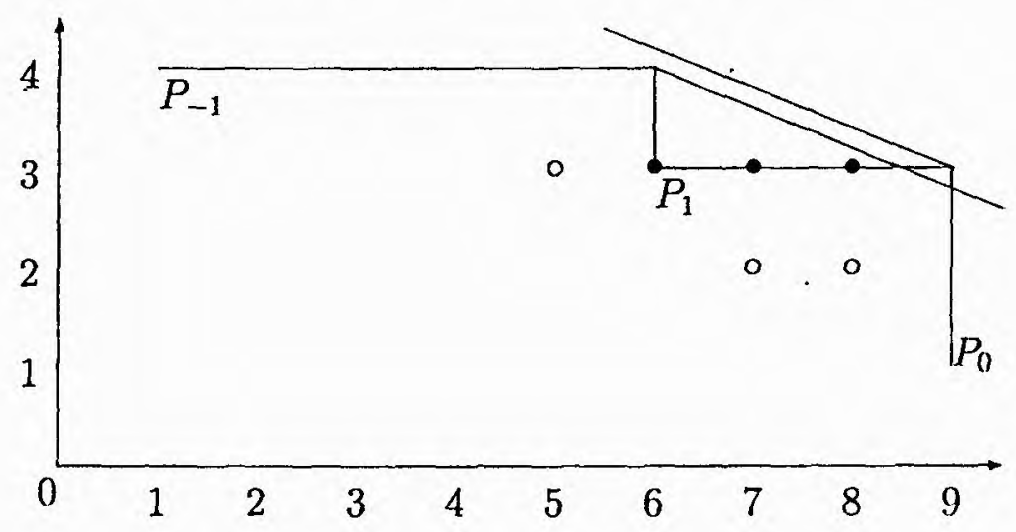

Como não há lacuna $l \notin L_{1}$ tal que $l>\min \left\{\varrho^{-1}(6,4), \varrho^{-1}(9,3)\right\}=$ $\min \{24,27\}$, o algoritmo finaliza. Dessa forma, para curvas da forma

$$
C:\left\{\begin{array}{l}
x=t^{4} \\
y=t^{9}+t^{11}+\sum_{i>11} a_{i} t^{i},
\end{array}\right.
$$

com $a_{i} \in K$ tomados genericamente, o conjunto de lacunas especiais é $\Lambda \backslash \Gamma=$ $L_{1}$, o menor valor de $\tau$ é $\mu-\sharp\left(L_{1}\right)=24-3=21$ e o diagrama de lacunas é o conjunto dos pontos assinalados na figura anterior.

b) Tome $\lambda$ mínimo, i.e., $\lambda=10=2 v_{1}-2 v_{0}$, assim $P_{1}=(8,2)$ e $L_{1}=$ $\{14,23\}$.

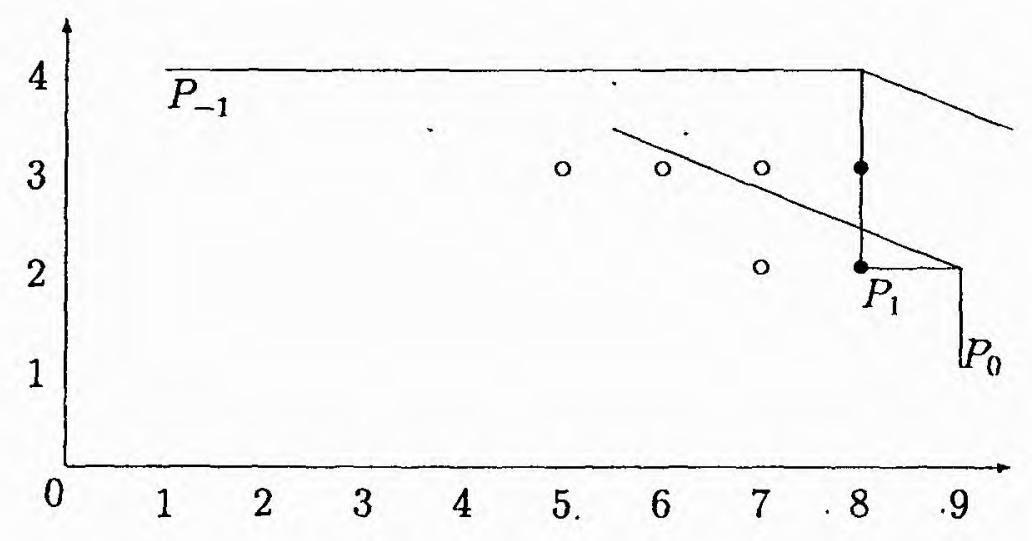

Note que, $19 \notin L_{1}$ e $19>\min \left\{\varrho^{-1}(8,4), \varrho^{-1}(9,2)\right\}=\min \{32,18\}$, assim podemos definir $P_{2}=\varrho(19)=(7,3)$ e $L_{2}=\{14,19,23\}$. 
No próximo passo, $\left(a_{2}, b_{2}\right)=(7,4) e\left(c_{2}, d_{2}\right)=(8,3)$, e desse modo, $\min \left\{\varrho^{-1}\left(a_{2}, b_{2}\right), \varrho^{-1}\left(c_{2}, d_{2}\right)\right\}=\min \{28,23\}$. Portanto, o algoritmo finaliza com o diagrama de lacunas como indicado na figura seguinte.

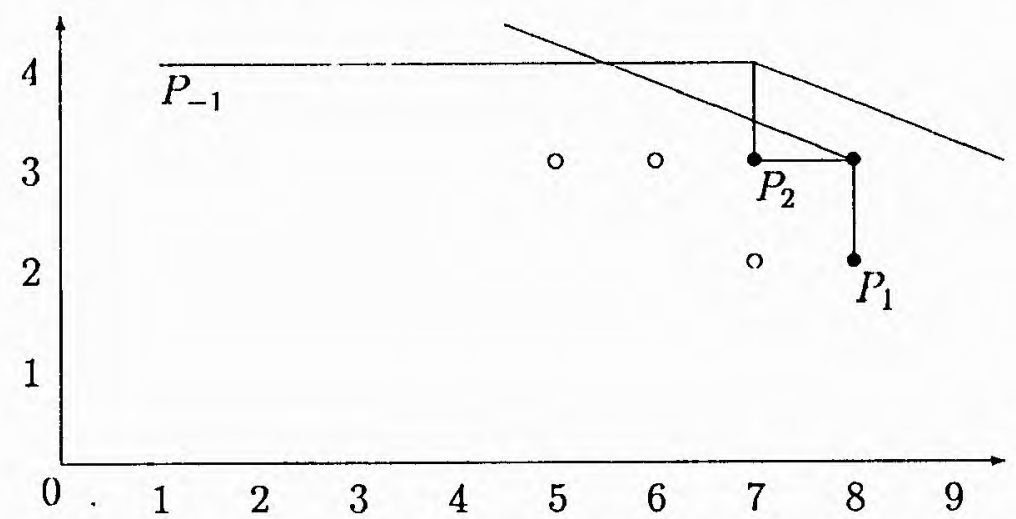

Portanto, o conjunto de lacunas especiais é $\Lambda \backslash \Gamma=L_{2}$ e o menor valor de $\tau$ para a classe de equisingularidade dada por $\Gamma=\langle 4,9\rangle$ é $\mu-\sharp\left(L_{2}\right)=$ $24-3=21$.

Mesmo quando não estamos na situação genérica, conhecendo apenas o semigrupo de valores e o invariante $\lambda$ de Zariski, podemos em alguus casos, ainda com o algoritmo acima, determinar as lacunas especiais e portanto $\tau$, como mostra o corolário abaixo.

Corolário 5.1 Seja C uma curva algebróide irredutivel plana com semigrupo $\Gamma=\left\langle v_{0}, v_{1}\right\rangle$ e invariante de Zariski $\lambda=i v_{1}-j v_{0} . S e$

$$
\left(2 i-v_{0}-1\right) v_{1}-2 v_{0}<\lambda<\left(2 i-v_{0}+1\right) v_{1},
$$

então existe apenas uma DNEM, a saber $\Omega_{1}=x d y-\frac{v_{1}}{v_{0}} y d x$. Além disso, $\sharp(\Lambda \backslash \Gamma)=\left(v_{0}-i\right)(j-1)$ e as lacunas especiais são $\left\{(i+\beta) v_{1}-(j-\alpha-1) v_{0}\right.$ com $0 \leq \beta \leq v_{0}-i-1$ e $\left.0 \leq \alpha \leq j-2\right\}$.

Dem.: A existência de $\Omega_{1}$ é garantida pela presença do invariante de Zariski $\lambda=i v_{1}-j v_{0}$ na parametrização de $C$.É óbvio que, se $\Omega_{1}$ é a única DNEM obtida pelo Algoritmo 2.2, então as ordens das DNE's. são da forma $v(\Omega)=$ $v\left(x^{\alpha} y^{\beta} \Omega_{1}\right)$, tais que $v(\Omega)+1=i v_{1}-(j-1) v_{0}+\alpha v_{0}+\beta v_{1}=(i+\beta) v_{1}-(j-$ $1-\alpha) v_{0}$ é uma lacuna, isto nos indica que $0 \leq \beta \leq v_{0}-i-1$ e $0 \leq \alpha \leq j-2$. 
Uma simples contagem mostra que nesse caso teremos $\left(v_{0}-i\right)(j-1)$ lacunas especiais.

Com as notações utilizadas no teoremn anterior temos que $\varrho^{-1}\left(c_{1}, d_{1}\right)=$ $i v_{1}$ e $\varrho^{-1}\left(a_{1}, b_{1}\right)=v_{0} v_{1}-(j-1) v_{0}$.

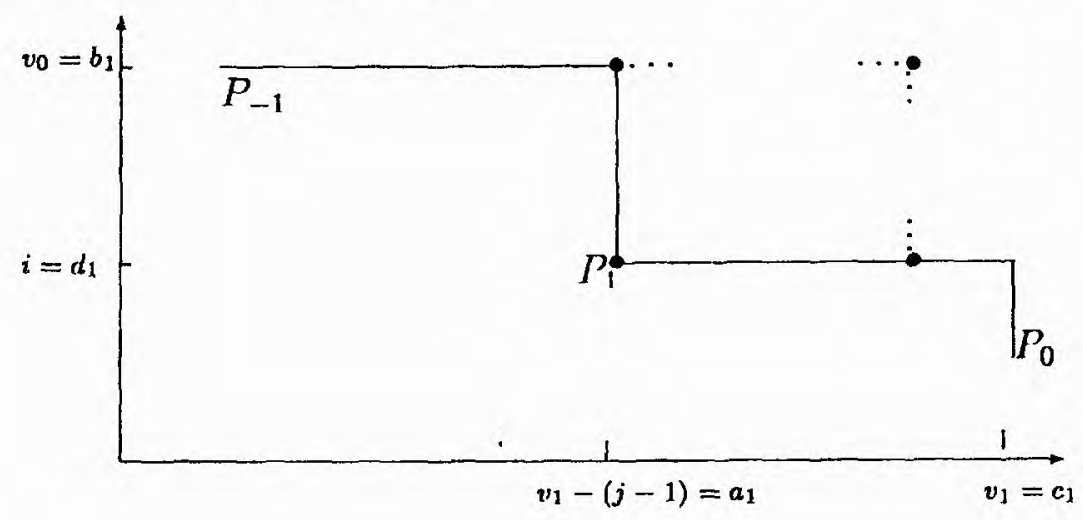

Temos dois casos a considerar:

Caso 1: $\varrho^{-1}\left(c_{1}, d_{1}\right)=i v_{1}>v_{0} \dot{v}_{1}-(j-1) v_{0}=\varrho^{-1}\left(a_{1}, b_{1}\right)$.

Por hipótese $\left(2 i-v_{0}-1\right) v_{1}-2 v_{0}<\lambda$, então $i v_{1}-v_{1}-v_{0}<v_{0} v_{1}-(j-1) v_{0}$ e assim $\varrho^{-1}\left(a_{1}, b_{1}\right)>\varrho^{-1}\left(c_{3}, d_{1}\right)-v_{1}-v_{0}$, mas dessa forma, não pode haver uma nova DNEM, finalizando o algọitmo (Veja figura abaixo).

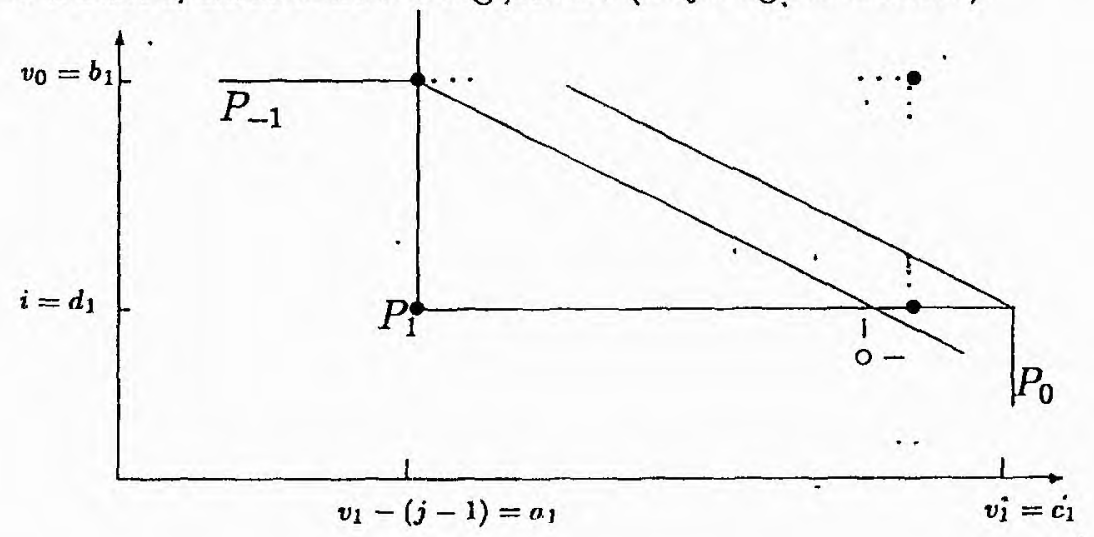

Caso 2: $\varrho^{-1}\left(a_{1}, b_{1}\right)=v_{0} v_{1}-(j-1) v_{0}>i v_{1}=\varrho\left(c_{1}, d_{1}\right)$.

Uma vez que $\lambda<\left(2 i-v_{0}+1\right) v_{1}$, cutão $v_{0} v_{1}-j v_{0}-v_{1}<i v_{1}<v_{0} v_{1}-$ $j v_{0}+v_{0}$, ou seja, $\varrho^{-1}\left(c_{1}, d_{1}\right)>\varrho^{-1}\left(a_{1}, b_{1}\right)-v_{1}-v_{0}$, novamente não há lacunas superiores à $\varrho^{-1}\left(a_{1}, b_{1}\right)$, com exceção das que são da forma $v\left(\Omega_{1}\right)+1+\gamma$ com $\gamma \in \Gamma$, isto garante o fim do algoritmo (Veja figura abaixo). 


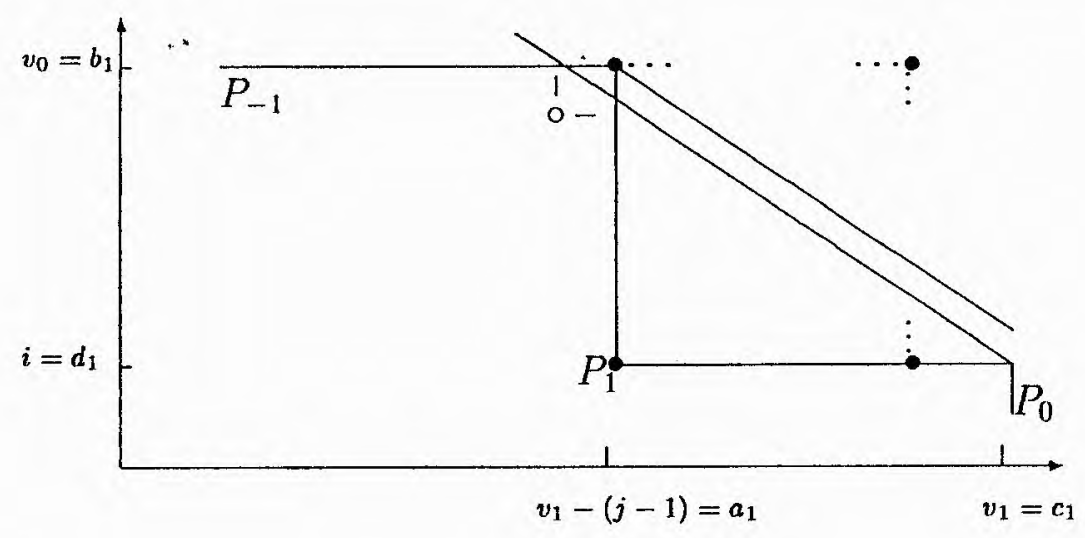

Exemplo 5.3 Considere $\Gamma=\left\langle 5, v_{1}\right\rangle$, com $v_{1}<10$. Uma ve. que. $\lambda>2 v_{1}-$ $2 v_{0}$, as únicas possibilidades para o invariante de Zariski são:

$$
\lambda=3 v_{1}-j v_{0} \text { ou } \lambda=4 v_{1}-j v_{0} .
$$

Se $\lambda$ é da forma $3 v_{1}-j v_{0}$, então $\lambda \leq 3 v_{1}-2 v_{0}$. Como $v_{1}<2 v_{0}$, temos que. $\lambda<2 v_{1}$, portanto estamos nas condiçôes do corolário anterior.

Por outro lado, se $\lambda=4 v_{1}-j v_{0}$, então $2 v_{1}-2 v_{0}<v_{1}<\lambda<4 v_{1}$, novamente satisfazendo as hipóteses do corolário.

Portanto, qualquer que seja o valor do invariante de Zariski, as curvas com semigrupo $\Gamma=\left\langle 5, v_{1}\right\rangle$, com $v_{1}<10$, admitem apenas uma DNEM.

Observação 5.3 A recíproca do corolário anterior não é verdadeira. De fato, existem ramos quie admitem apenas $\Omega_{1}=x d y-\frac{v_{1}}{v_{0}} y d x$ como DNEM $e$ possuem invariante de Zariski fora do intervalo dado no corolário anterior. Por exemplo, considere

$$
C:\left\{\begin{array}{l}
x=t^{4} \\
y=t^{9}+t^{10}+a t^{11}
\end{array}\right.
$$

Como $\lambda=10=2 v_{1}-2 v_{0}>9=\left(2 i-v_{0}+1\right) v_{1}$, estamos fora das condições do corolário anterior. No entanto, aplicando o Algoritmo 2.2 temos as seguintes possibilidades: 
- Se $a \neq \frac{19}{18}$, então existem duas DNEM's, a saber $\Omega_{1}=x d y-\frac{9}{4} y d x$ e $\Omega_{2}=x \Omega_{1}-\frac{1}{9} y d y \operatorname{com} v\left(\Omega_{1}\right)+1=14$ e $v\left(\Omega_{2}\right)+1=19$.

- Se $a=\frac{19}{18}$, ent.ão exist.e apenas a $D N E M \Omega_{1}=x d y-\frac{9}{4} y d x$.

Na verdade, fuxado $\Gamma=\langle 4,9\rangle, 10$ é o único valor de $\lambda$ para o qual $C$ pode admitir mais de uma DNEM. De fato, os possiveis valores de $\lambda$ são:

$$
\begin{gathered}
\lambda=2 v_{1}-j v_{0} \Longrightarrow \lambda=10 \\
\lambda=3 v_{1}-j v_{0} \Longrightarrow \lambda \in\{11,15,19\} .
\end{gathered}
$$

Se $\lambda=3 v_{1}-j v_{0}$, i.e., $i=3$, então a condição do corolário anterior é satisfeita e portanto $C$ admite apenas $\Omega_{1}$ como DNEM.

O Corolário 5.1 é útil para efetuar o estudo completo do diagrama de lacunas para uma classe de equisingularidade fixa em gênero 1 , como podemos ver no exemplo a seguir.

Exemplo 5.4 Considere $\Gamma=\langle 5,11\rangle$. Os possiveis valores de $\lambda$ säo:

$$
\begin{gathered}
\lambda=2 v_{1}-j v_{0} \Longrightarrow \lambda=12 \\
\lambda=3 v_{1}-j v_{0} \Longrightarrow \lambda \in\{13,18,23\} \\
\lambda=4 v_{1}-j v_{0} \Longrightarrow \lambda \in\{14,19,24,29,34\}
\end{gathered}
$$

O Corolário 5.1 permite afirmar que, se $\lambda \in\{13,14,18,19,24,29,34\}$, então existe apenas $\Omega_{1}=x d y-\frac{11}{5} y d x$ como DNEM, e temos:

\begin{tabular}{|c|c|c|}
\hline$\lambda$ & lacun.as & $\sharp(\Lambda \backslash \Gamma)$ \\
\hline 13 & $18,23,28,29,34,39$ & 6 \\
14 & $19,24,29,34,39$ & 5 \\
18 & $23,28,34,39$ & 4 \\
19 & $24,29,34,39$ & 4 \\
24 & $29,34,39$ & 3 \\
29 & 34,39 & 2 \\
34 & 39 & 1. \\
\hline
\end{tabular}


Resta analisarmos os casos $\lambda=12$ e $\lambda=23$.

Se $\lambda=23$, então pelo Teorema 4.1; podemos considerar

$$
C:\left\{\begin{array}{l}
x=t^{5} \\
y=t^{11}+t^{23}+a t^{24}+b t^{29}
\end{array}\right.
$$

Como $\Omega_{1}=x d y-\frac{11}{5} y d x$ e $\psi\left(\Omega_{1}\right)=12 t^{27}+13 a t^{28}+18 b t^{33}$, temos que $v\left(\Omega_{1}\right)+1=28, v\left(y \Omega_{1}\right)+1=39$ e assim a lacuna limitante é $l=34$.

$O$ próximo $S$-processo mínimo é $\Omega_{2}=x \Omega_{1}-\frac{12}{11} y^{2} d y$ e $\psi\left(\Omega_{1}\right)=13 a t^{33}+$ $18 b t^{38}+\cdots$.

Surgem as seguintes possibilidades:

Se $a \neq 0$, ent.ão $\Omega_{2}$ é uma nova DNEM, $v\left(\Omega_{2}\right)+1=34$ o: a lacuna limitante é $l=29$, indicando o fim do algorit.mo.

Se $a=0$, então o algoritmo finaliza.

Resumindo o caso $\lambda=23$, tem.os

\begin{tabular}{|c|c|c|}
\hline$a$ & lacunas & $\sharp(\Lambda \backslash \Gamma)$ \\
\hline$=0$ & 28,39 & 2 \\
$\neq 0$ & $28,34,39$ & 3 \\
\hline
\end{tabular}

Analisemos agora o caso em que $\lambda=12$. Eliminando os termos em $\Gamma \cup\left\{\Gamma-v_{1}+v_{0}\right\} \cup\left\{\Gamma^{*}+\lambda\right\}$, como no Teorema 4.1, podemos considerar

$$
C:\left\{\begin{array}{l}
x=t^{5} \\
y=t^{11}+t^{12}+a t^{13}+b t^{14}+c t^{18}+d t^{19}+e t^{24}+f t^{29}
\end{array}\right.
$$

Uma vez que, $\Omega_{1}=x d y-\frac{11}{5} y d x$ e $\psi\left(\Omega_{1}\right)=t^{16}+2 a t^{17}+3 b t^{18}+7 c t^{22}+$ $8 d t^{23}+13 e t^{28}+18 f t^{33}, v\left(\Omega_{1}\right)+1=17, v\left(y \Omega_{1}\right)+1=28$ e $v\left(\Omega_{1}\right)+1=39$, temos que $l=34$ é a lacuna limitante.

O próximo $S$-processo mínimo a ser considerado é

$$
\Omega_{2}=11 x \Omega_{1}-y d y
$$

$\operatorname{com} \psi\left(\Omega_{2}\right)=(22 a-23) t^{22}+(33 b-12-24 a) t^{23}-25(a+b) t^{24}-13\left(a^{2}+b\right) t^{25}+\cdots$

Temos dois casos a analisar:

1. $a \neq \frac{23}{22}$ :

Neste caso, $\Omega_{2} \cdot$ é DNEM. Uma vez que $v\left(\Omega_{2}\right)+1=23$ e $v\left(y \Omega_{2}\right)+1=34$, a lacuna limitante passa a ser $l=29$. No próximo passo, consideramos 
o $S$-processo mínimo $\Omega_{3}=x \Omega_{2}-(22 a-23) y \Omega_{1}$, desse modo, temos $\psi\left(\Omega_{3}\right)=11\left(3 b-4 a^{2}+1\right) t^{28}+11\left(4 b+4 a-6 a^{2}-6 a b\right) t^{29}+\cdots$

$S e b \neq \frac{(2 a+1)(2 a-1)}{3}$, então $\Omega_{3}$ é uma nova DNEM. Comov $\left(\Omega_{3}\right)+1=29$, a lacuna limitante passa a ser $l=24$, indicando o fim do algoritmo. Por outro lado, se $b=\frac{(2 a+1)(2 a-1)}{3}$, então o algoritmo finaliza com $\Omega_{1}$ e $\Omega_{2}$.

2. $a=\frac{23}{22}$ :

Temos $\psi\left(\Omega_{2}\right)=33\left(b-\frac{136}{121}\right) t^{2.3}+\left(-\frac{575}{22}-25 b\right) t^{24}+\cdots$, e as seguinte.s possibilidades:

(a) $b \neq \frac{136}{121}$ :

Tem.os que $\Omega_{2}$ é uma nova DNEM, com $v\left(\Omega_{2}\right)+1=24, v\left(x \Omega_{2}\right)+$ $1=29, v\left(x^{2} \Omega_{2}\right)+1=34$ e a lacuna limitante él $l=23$, indicando o fim do algoritmo.

(b) $b=\frac{136}{121}$ :

Neste caso, podemos reduzir $\Omega_{2}$ de forma a obtermos:

$\Omega_{2}^{\prime}=\Omega_{2}+\frac{2625}{242} x^{4} d x+\frac{1911}{220} y x^{2} d x-\frac{62319}{26620} y^{2} d x-\left(\frac{504725}{117128}+77 c\right) y \Omega_{1}$, com $\psi\left(\Omega_{2}^{\prime}\right)=A t^{28}+\cdots$, onde $A=\left(88 d-\frac{440215}{644204}-267 c\right)$.

Podemos ter:

i. $A \neq 0$ :

Temos que $\Omega_{2}^{\prime}$ é DNEM com $v\left(\Omega_{2}^{\prime}\right)+1=29, v\left(x \Omega_{2}^{\prime}\right)+1=$ 34 e a lacuna limitante é $l=24$, indicando que o algortimo finaliza.

ii. $A=0$ :

Nesta situação podemos reduzir $\Omega_{2}^{\prime}$. A saber, sua redução final. é:

$$
\begin{aligned}
\Omega_{2}^{\prime \prime}= & \Omega_{2}^{\prime}+\frac{1}{5}\left(\frac{195442184125}{2522702864}+\frac{18250}{89} d\right) x^{5} d x+ \\
& +\frac{1}{5}\left(\frac{251638551583}{3784054296}+\frac{56405}{267} d\right) y x^{3} d x+ \\
& -\frac{1}{5}\left(\frac{621173591305}{41624597256}+\frac{49463}{2937} d\right) y^{2} x d x+ \\
& -\frac{1}{11}\left(\frac{4209205750711}{457870569816}+\frac{1407769}{64614} d\right) y^{2} d y
\end{aligned}
$$

com $\psi\left(\Omega_{2}^{\prime \prime}\right)=B t^{33}+\cdots$, onde

$$
B=\left(\frac{1807239518543737}{5379063454198368}-\frac{4174016}{71289} d^{2}+143 e+\frac{462825631}{379542636} d\right)
$$


Temos as possibilidades:

- $B \neq 0$ :

Temos que $\Omega_{2}^{\prime \prime}$ é uma DNEM, $v\left(\Omega_{2}^{\prime \prime}\right)+1=34$ e o algorit.mo finaliza.

- $B=0$ :

Neste caso o algoritmo finaliza com uma única DNEM, a saber $\Omega_{1}$.

Resumindo o estudo para $\lambda=12$, temos

\begin{tabular}{|c|c|c|c|c|}
\hline \multirow[t]{2}{*}{$a \neq \frac{23}{22}$} & \multicolumn{3}{|c|}{$b \neq \frac{(2 a+1)(2 a-1)}{3}$} & $\Lambda \backslash \Gamma=\{17,23,28,29,34,39\}$ \\
\hline & \multicolumn{3}{|c|}{$b=\frac{(2 a+1)(2 a-1)}{3}$} & $\Lambda \backslash \Gamma=\{17,23,28,34,39\}$ \\
\hline \multirow{4}{*}{$a=\frac{23}{22}$} & \multicolumn{3}{|l|}{$b \neq \frac{136}{121}$} & $\Lambda \backslash \Gamma=\{17,24,28,29,34,39\}$ \\
\hline & \multirow{3}{*}{$b=\frac{136}{121}$} & $A \neq 0$ & & $\Lambda \backslash \Gamma=\{17,28,29,34,39\}$ \\
\hline & & \multirow[t]{2}{*}{$A=0$} & $\bar{B} \neq 0$ & $\Lambda \backslash \Gamma=\{17,28,34,39\}$ \\
\hline & & & $B=0$ & $\Lambda \backslash \Gamma=\{17,28,39\}$ \\
\hline
\end{tabular}

Façamos agora o resumo para as possibilidades de $\sharp(\Lambda \backslash \Gamma)$ e o diagrama de lacunas. Como referencial tomamos o ponto que se localiza no canto superior direito como a lacuna $\left(v_{0}-1\right) v_{1}-v_{0}=\mu-1=39$.

\begin{tabular}{|c|c|c|c|}
\hline$H(\Lambda \backslash \Gamma)$ & $\lambda$ & lacunas & diagrama \\
\hline 1 & 34 & 39 & - \\
\hline \multirow[t]{2}{*}{2} & 29 & 34,39 & $\bullet \bullet$ \\
\hline & 23 & 28,39 & $\bullet$ \\
\hline \multirow{3}{*}{3} & 24 & $29,34,39$ & $\bullet \bullet \bullet$ \\
\hline & 23 & $28,34,39$ & $\bullet \bullet$ \\
\hline & 12 & $17,28,39$ & $\bullet$ \\
\hline \multirow{3}{*}{4} & 19 & $24,29,34,39$ & $\bullet \bullet \bullet \bullet$ \\
\hline & 18 & $23,28,34,39$ & $\bullet \quad \bullet$ \\
\hline & 12 & $17,28,34,39$ & $\bullet \bullet$ \\
\hline
\end{tabular}




\begin{tabular}{|c|c|c|c|}
\hline & 14 & $19,24,29,34,39$ & $\bullet \bullet, \bullet \bullet \bullet$ \\
\hline \multirow[t]{2}{*}{5} & 12 & $17,28,29,34,39$ & $\bullet$ \\
\hline & 12 & $17,23,28,34,39$ & $\begin{array}{l}- \\
-\end{array}$ \\
\hline \multirow{3}{*}{6} & 13 & $18,23,28,29,34,39$ & $\bullet \bullet \bullet$ \\
\hline & 12 & $17,24,28,29,34,39$ & $\bullet \bullet \bullet$ \\
\hline & 12 & $17,23,28,29,34,39$ & - \\
\hline
\end{tabular}

\subsection{Ramo $\lambda$-canônico}

Como já observamos, na seção anterior, se $C$ é uma curva algêbróide irredutível plaua de gênero 1 e $\lambda$ seu invariante de Zariski, então $C$ pode ser dada por uma parametrização da forma

$$
C:\left\{\begin{array}{l}
x=t^{v_{0}} \\
y=t^{v_{1}}+t^{\lambda}+\sum_{i>\lambda} a_{i} t^{i} .
\end{array}\right.
$$

Se $a_{i}=0$ para todo $i>\lambda$, então o invariante $\lambda$ determina completamente a classe de equivalência de $C$. Chamaremos a curva

$$
C:\left\{\begin{array}{l}
x=t^{v_{0}} \\
y=t^{v_{1}}+t^{\lambda}
\end{array}\right.
$$

de ramo $\lambda$-can.ônico associado ao semigrupo $\Gamma=\left\langle v_{0}, v_{1}\right\rangle$.

Uma questão natural que surge, é saber qual o valor de $\tau$, on mais especificamente, qual o conjunto de lacunas especiais para curvas $\lambda$-canônicas.

Carbonne, em [Car], apresenta a segụinte proposição:

\section{Proposição 5.1 Seja}

$$
C:\left\{\begin{array}{l}
x=t^{v_{0}} \\
y=t^{v_{1}}+t^{\lambda}
\end{array}\right.
$$


$\operatorname{com} M D C\left(v_{0}, v_{1}\right)=1, v_{0}<v_{1}<\lambda e \lambda=\left(v_{0}-1\right) v_{1}-j v_{0} \operatorname{com} j=2, \ldots,\left[\frac{v_{1}}{v_{0}}\right]$, entâo $\tau=\mu-(j-1)$ e o conjunto das lacunas especiais é $\Lambda \backslash \Gamma=\left\{\lambda+i \nu_{0} ; 1 \leq\right.$ $i \leq j-1\}$.

Dem.: Segue diretamente do Corolário 5.1.

Vamos aplicar o Algoritmo 2.2 para encontrar uma Base Standard Mínima de $\mathcal{O} d \mathcal{O}$, obtendo uma fórmula explícita para as DNEM's, bem como para $\sharp(\Lambda \backslash \Gamma)$ quando $C$ é um ramo $\lambda$-cainônico com invariante de Zariski $\lambda=$ $\left(v_{0}-1\right) v_{1}-j v_{0}$, com $j=2, \ldots,\left[\frac{\left(v_{0}-2\right) v_{1}}{v_{0}}\right]$, i.e., para $\lambda \equiv\left(v_{0}-1\right) v_{1} \bmod v_{0}$.

Considerando o homomorfismo de $\mathcal{O}$-módulos $\psi$ definido em (2.2), no primeiro passo do Algoritmo 2.2 obtemos

$$
\Omega_{1}=x d y-\frac{v_{1}}{v_{0}} y d x
$$

$\operatorname{com} \psi\left(\Omega_{1}\right)=\left(\lambda-v_{1}\right) t^{\lambda+v_{0}-\mathrm{i}}$.

Note que temos $j-1$ lacunas especiais da forma $v\left(x^{k} \Omega_{1}\right)+1 \operatorname{com} k=$ $0, \ldots, j-2$.

Sem perda de generalidade, podemos considerar $\Omega_{1}=\frac{1}{\lambda-v_{1}}\left(x d y-\frac{v_{1}}{v_{0}} y d x\right)$, $\operatorname{assim} \psi\left(\Omega_{1}\right)=t^{\lambda+v_{0}-1}$.

O lema abaixo nos auxiliará a comparar lacunas.

Lema 5.1 Seja $\operatorname{MDC}\left(v_{0}, v_{1}\right)=1$ e $a, b, c, d \in \mathbb{N}$. Temos que $a v_{1}-b v_{0}>$ $(a-d) v_{1}-c v_{0}$ se, e somente se, $b-c \leq\left[\frac{d v_{1}}{v_{0}}\right]$.

Dem.: De fato, $a v_{1}-b v_{0}>(a-d) v_{1}-c v_{0}$ é equivalente. a $b v_{0}<d v_{1}+c v_{0}$, ou seja, $b-c \leq\left[\frac{d v_{1}}{v_{0}}\right]$.

Agora observe que se $v_{0}=3$, então as lacunas especiais são aquelas maiores ou iguais a $\lambda+v_{0}=\left(v_{0}-1\right) v_{1}-(j-1) v_{0}$, ou seja, da forma $\left(v_{0}-1\right) v_{1}^{\prime}-(j-k-1) v_{0}$ com $0 \leq k \leq j-2$.

Se $v_{0}>3$, então toda lacuna maior ou igual a $\left(v_{0}-1\right) v_{1}-\gamma v_{0}$ com $\gamma=\min \left\{j,\left[\frac{v_{1}}{v_{0}}\right]+1\right\}$ é especial. Realmente, se $v_{0}>3$, então existem lacunas maiores que $v_{1}$ da forma $\left(v_{0}-2\right) v_{1}-k v_{0}, \operatorname{com} k=1, \ldots,\left[\frac{\left(v_{0}-3\right) v_{1}}{v_{0}}\right]$. Pelo 
lema anterior, temos que $\left(v_{0}-1\right) v_{1}-\gamma v_{0}>\left(v_{0}-2\right) v_{1}-v_{0}$ se, e somente se, $\gamma-1 \leq\left[\frac{v_{1}}{v_{0}}\right]$.

Assim, independente do valor de $v_{0}$, toda lacuna maior ou igual a $\left(v_{0}\right.$ 1) $v_{1}-\gamma v_{0} \operatorname{com} \gamma=\min \left\{j,\left[\frac{v_{1}}{v_{0}}\right]+1\right\}$ é especial e é obtida no primeiro passo do algoritmo.

A Proposição 3.3 , indica que o próximo $S$-processo mínimo de $B=$ $\left\{d x, d y, \Omega_{1}\right\}$ a ser considerado é

$$
\Omega_{2}=y \Omega_{1}-\frac{1}{v_{0}} x^{v_{1}-j} d x
$$

onde $\psi\left(\Omega_{2}\right)=t^{2 \lambda+v_{0}-1}$.

Note que $2 \lambda+v_{0}=\left(v_{0}-2\right) v_{1}+\left(v_{1}-2 j+1\right) v_{0}$.

Se $j<\frac{v_{1}+2}{2}$, eutão $2 \lambda+v_{0} \in \Gamma$ e como $2 \lambda+v_{0}>\left(v_{0}-2\right) v_{1}-v_{0}$, a redução final de $\Omega_{2}$ módulo $B$ e $F=\{x, y\}$ é zero.

Assim, se

$$
C:\left\{\begin{array}{l}
x=t^{v_{0}} \\
y=t^{v_{1}}+t^{\left(v_{0}-1\right) v_{1}-j v_{0}}
\end{array}\right.
$$

com $2 \leq j<\frac{v_{1}+2}{2}$ e $v_{0} \geq 3$, ent,ão $\sharp(\Lambda \backslash \Gamma)=j-1$.

Para continuar a análise consideramos $\frac{v_{1}+2}{2} \leq j$ e $v_{0} \geq 4$. Dessa forma, $2 \lambda+v_{0}=\left(v_{0}-2\right) v_{1}-\left(2 j-v_{1}-1\right) v_{0}$ é lacuna, assim $\Omega_{2}$ é uma nova DNEM e temos $2 j-v_{1}-1$ novas lacuuas especiais da forma $v\left(x^{k} \Omega_{2}\right)+1=$ $2 \lambda+v_{0}+k v_{0}=\left(v_{0}-2\right) v_{1}-\left(2 j-v_{1}-1-k\right) v_{0}$ com $k=0, \ldots, 2 j-v_{1}-2$.

Se $v_{0}=4$, então todas as lacunas maiores que $v_{1}$ são da forma $\left(v_{0}-1\right) v_{1}-$ $\alpha v_{0}, \operatorname{com} \alpha=1, \ldots,\left[\frac{\left(v_{0}-2\right) v_{1}}{v_{0}}\right]$ ou $\left(v_{0}-2\right) v_{1}-\beta v_{0}, \operatorname{com} \beta=1, \ldots,\left[\frac{\left(v_{0}-3\right) v_{1}}{v_{0}}\right]$. Desse modo, toda lacuna maior ou igual a $\left(v_{0}-2\right) v_{1}-\left(2 j-v_{1}-1\right) v_{0}$ é especial e o algoritmo finaliza.

Se $v_{0}>4$, então todas as lacunas maiores on iguais a $\left(v_{0}-2\right) v_{1}-\gamma v_{0}$ com $\gamma=\min \left\{2 j-v_{1}-1,\left[\frac{v_{1}}{v_{0}}\right]\right\}$ é lacuna especial. De fato, uma vez que existem lacunas acima de $v_{1}$, da forma $\left(v_{0}-3\right) v_{1}-k v_{0} \operatorname{com} k=1, \ldots,\left[\frac{\left(v_{0}-4\right) v_{1}}{v_{0}}\right]$, o lema anterior nos indica que $\left(v_{0}-2\right) v_{1}-\gamma v_{0}>\left(v_{0}-3\right) v_{1}-v_{0}$ se, e somente se, $\gamma \leq\left[\frac{v_{1}}{v_{0}}\right]+1$

Assim, para qualquer valor de $v_{0} \geq 4$, toda lacuna maior ou igual a $\left(v_{0}-2\right) v_{1}-\gamma v_{0} \operatorname{com} \gamma=\min \left\{2 j-v_{1}-1,\left[\frac{v_{1}}{v_{0}}\right]+1\right\}$ é obtida após o segundo passo do algoritmo.

Se $v_{0}>4$, então o próximo $S$-processo mínimo dẹ $B^{\prime}=\left\{d x, d y, \Omega_{1}, \Omega_{2}\right\}^{\prime}$ que merece análise é

$$
\Omega_{3}=y \Omega_{2}-x^{v_{1}-j} \Omega_{1}
$$


onde $\psi\left(\Omega_{3}\right)=t^{3 \lambda+v_{0,-1}}$.

Note que $3 \lambda+v_{0}=\left(v_{0}-3\right) v_{1}-\left(3 j-2 v_{1}-1\right) v_{0}$. Se $j<\frac{2 v_{1}+2}{3}$, então $3 \lambda+v_{0} \in \Gamma$ e a redução de $\Omega_{3}$ só poderá nos dar uma lacuua especial já obtida, ou seja, a redução fiual de $\Omega_{3}$ módulo $(B, F)$ será zero, indicaudo o fim do algoritmo, onde $F=\{x, y\}$. Dessa forma, se

$$
C:\left\{\begin{array}{l}
x=t^{v_{0}} \\
y=t^{v_{1}}+t^{\left(v_{0}-1\right) v_{1}-j v_{0}}
\end{array}\right.
$$

com $\frac{v_{1}+2}{2} \leq j<\frac{2 v_{1}+2}{3}$ e $v_{0} \geq 4$, então $\sharp(\Lambda \backslash \Gamma)=j-1+2 j-v_{1}-1=3 j-v_{1}-2$.

O lema abaixo, corresponde ao passo indutivo das afirmações acima.

Lema 5.2 Se $\Omega_{i}$ é DNEM com $v\left(\Omega_{i}\right)+1=i \lambda+v_{0}$ com $i=1, \ldots, k$ para $\frac{(k-1) v_{1}+2}{k} \leq j$ e $v_{0} \geq 2+k$, então toda lacuna maior ou igual a $\left(v_{0}-k\right) v_{1}-\gamma v_{0}$ $\operatorname{com} \gamma=\min \left\{k j-(k-1) v_{1}-1,\left[\frac{v_{1}}{v_{0}}\right]+1\right\}$ é lacuna especial.

Dem.: Note que o lema já foi verificado para $k=1$ e $k=2$.

Se $v_{0}=k+2$, então não temos lacunas maiores que $v_{1}$ da forma $\left(v_{0}-k-\right.$ 1) $v_{1}-\alpha v_{0}$. Como $v\left(\Omega_{k}\right)+1=\left(v_{0}-k\right) v_{1}-\left(k j-(k-1) v_{1}-1\right) v_{0}$ é lacuna especial e $v\left(\Omega_{i-1}\right)<v\left(\Omega_{i}\right)$, todas as lacunas maiores ou iguais a $v\left(\Omega_{k}\right)+1$ são. especiais. Note que $k j-(k-1) v_{1}-1 \leq\left[\frac{v_{1}}{v_{0}}\right]$.

Se $v_{0}>2+k$, então podem existir lacunas maiores que $v_{1}$ da forma $\left(\dot{v_{0}}-k-1\right) v_{1}-\alpha v_{0} \operatorname{com} \alpha=1, \ldots,\left[\frac{\left(v_{0}-k-2\right) v_{1}}{v_{0}}\right]$. Pelo lema anterior, $\left(v_{0}-\right.$ k) $v_{1}-\gamma v_{0}>\left(v_{0}-k-1\right) v_{1}-v_{0}$ se, e somente se, $\gamma \leq\left[\frac{v_{1}}{v_{0}}\right]+1$.

Novamente, como $v\left(\Omega_{i-1}\right)<v\left(\Omega_{i}\right)$, temos que toda lacuna maior on igual a $\left(v_{0}-k\right) v_{1}-\gamma v_{0} \operatorname{com} \gamma=\min \left\{k j-(k-1) v_{1}-1,\left[\frac{v_{1}}{v_{0}}\right]+1\right\}$ é especial.

No desenrolar da prova do lema anterior, vemos que se $v_{0}=2+k$, então o algoritmo finaliza. Se $v_{0}>2+k$ e $j \geq \frac{(k-1) v_{1}+2}{k}$, então a Proposição 3.3 indica que o próximo $S$-processo mínimo a ser considerado é

$$
\Omega_{k+1}=y \Omega_{k}-x^{v_{1}-j} \Omega_{k-1} .
$$

Suponha que $\Omega_{i}=t^{i \lambda+v_{0}-1}$ para $1 \leq i \leq k$, assim $\psi\left(\Omega_{k+1}\right)=t^{(k+1) \lambda+v_{0}-1}$.

Note que $v\left(\Omega_{k+1}\right)+1=(k+1) \lambda+v_{0}=\left(v_{0}-(k+1)\right) v_{1}-\left((k+1) j-k v_{1}-1\right) v_{0}$. Desse modo, se $(k+1) j-k v_{1}-1<1$, então $(k+1) \dot{\lambda}+v_{0} \in \Gamma$, mais ainda, como $(k+1) \lambda+v_{0}>\left(v_{0}-k\right) v_{1}-\gamma v_{0} \operatorname{com} \gamma=\min \left\{k j-(k-1) v_{1}-1,\left[\frac{v_{1}}{v_{0}}\right]+1\right\}$ e o 
$S$-processo acima possui redução final zero módulo $\left\{d x, d y, \Omega_{1}, \ldots, \Omega_{k}\right\}$ e $F$, o que indica o fim do algoritmo. Observe que a condição $(k+1) j-k v_{1}-1<1$ é equivalente a $j<\frac{k v_{1}+2}{k+1}$.

Por outro lado, se $j \geq \frac{k v_{1}+2}{k+1}$, então $\Omega_{k+1}$ é DNEM e temos $(k+1) j-k v_{1}-1$ novas lacunas especiais da forma $v\left(x^{\alpha} \Omega_{k+1}\right)+1 \operatorname{com} \alpha=0, \ldots,(k+1) j-$ $k v_{1}-2$.

Dessa forma, temos a seguinte proposição.

Proposição 5.2 Seja

$$
C:\left\{\begin{array}{l}
x=t^{v_{0}} \\
y=t^{v_{1}}+t^{\left(v_{0}-1\right) v_{1}-j v_{0}} .
\end{array}\right.
$$

Se $v_{0} \geq 2+k e^{\frac{(k-1) v_{1}+2}{k}} \leq j<\frac{k v_{1}+2}{k+1} \operatorname{com} k>0$, então $\sharp(\Lambda \backslash \Gamma)=$ $k\left(v_{1}-1-\frac{\left(v_{1}-j\right)(k+1)}{2}\right)$. Além disso temos $k$ DNEM's, a.saber

$$
\begin{gathered}
\Omega_{1}=\left(\frac{1}{\lambda-v_{1}}\right)\left(x d y-\frac{v_{1}}{v_{0}} y d x\right) \operatorname{com} \psi\left(\Omega_{1}\right)=t^{\lambda+v_{0}-1}, \\
\Omega_{2}=y \Omega_{1}-\frac{1}{v_{0}} x^{v_{1}-j} d x \operatorname{com} \psi\left(\Omega_{2}\right)=t^{2 \lambda+v_{0}-1}, \\
\Omega_{i}=y \Omega_{i-1}-x^{v_{1}-j} \Omega_{i-2} \operatorname{com} \psi\left(\Omega_{i}\right)=t^{i \lambda+v_{0}-1} \text { e } 3 \leq i \leq k .
\end{gathered}
$$

Assim, $v\left(\Omega_{i}\right)+1=i \lambda+v_{0}=\left(v_{0}-i\right) v_{1}-\left(i j-(i-1) v_{1}-1\right) v_{0}$ com $1 \leq i \leq k$ e o diagrama de lacunas é dado por

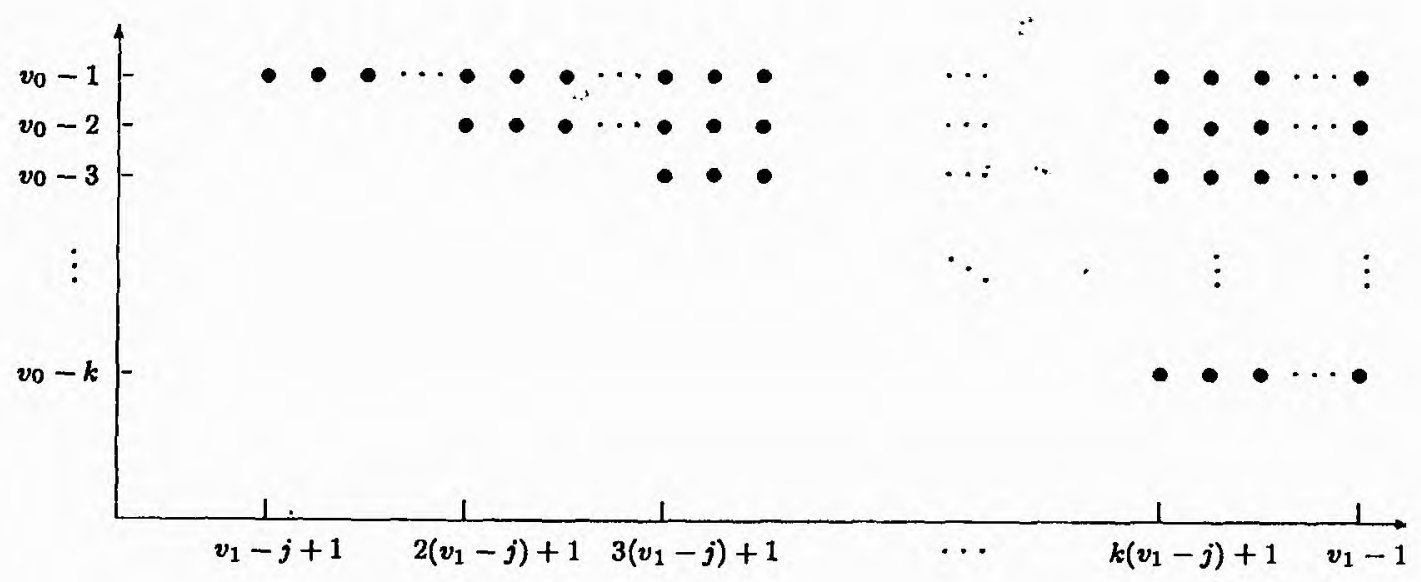


Note que, se $k=1$, então $2 \leq j<\frac{v_{1}}{2}+1$. Como $\left[\frac{v_{1}}{v_{0}}\right]<\frac{v_{1}}{2}+1$, temos como caso particular a Proposição 5.1 .

Além dos ramos $\lambda$-canônicos, $\operatorname{com} \lambda=\left(v_{0}-1\right) v_{1}-j v_{0}$, podemos obter a descrição completa das DNEM's e do conjunto de lacunas especiais para os ramos $\lambda$-canônicos $\operatorname{com} \lambda=2 v_{1}-j v_{0}$, i.e., para ramos da forma

$$
C:\left\{\begin{array}{l}
x=t^{v_{0}} \\
y=t^{v_{1}}+t^{2 v_{1}-j v_{0}}
\end{array}\right.
$$

Note que para $2 v_{1}-j v_{0}$ ser efetivamente o invariant,e de Zariski, devemos ter $j \geq 2$ e $2 v_{1}-j v_{0}>v_{1}$, on seja, $j \leq\left[\frac{v_{1}}{v_{0}}\right]$. Assim, estamos considerando $2 \leq j \leq\left[\frac{v_{1}}{v_{0}}\right]$, em particular, devemos ter $v_{1}>2 v_{0}$ e $v_{0}>2$.

Proposição 5.3 Seja

$$
C:\left\{\begin{array}{l}
x=t^{v_{0}} \\
y=t^{v_{1}}+t^{\lambda},
\end{array}\right.
$$

com $\lambda=2 v_{1}-j v_{0}, 2 \leq j \leq\left[\frac{v_{1}}{v_{0}}\right]$ e. $v_{0}>2$.

$O$ número de lacunas especiais de $C$ é $\sharp(\Lambda \backslash \Gamma)=\left[\frac{v_{0}-1}{2}\right]\left(\left[\frac{v_{0}}{2}\right] j-1\right)$, das quais $v_{0}-2$ correspondem a DNEM's. Se $\Omega_{-1}=\frac{d x}{v_{0}}$ e $\Omega_{0}=d y$, então as $D N E M$ 's são dadas, paru $1 \leq i \leq\left[\frac{v_{0}-1}{2}\right]$, por

$$
\Omega_{2 i-1}=-\frac{1}{(i-2) \lambda+v_{1}}\left(x \Omega_{2(i-1)}-\left((i-1) \lambda+v_{1}\right) y \Omega_{2(i-1)-1}\right),
$$

$\operatorname{com} \psi\left(\Omega_{2 i-1}\right)=t^{i \lambda+v_{1}-1} e$

$$
\Omega_{2 i}=y \Omega_{2(i-1)}-((i-1) \lambda+v-1) x \Omega_{2 i-1},
$$

$\operatorname{com} \psi\left(\Omega_{2 i}\right)=\left(i \lambda+v_{1}\right) t^{i \lambda+v_{1}-1}+\lambda t^{(i+1) \lambda-1}$.

Dem.: Vamos usar a Proposição 3.3 que nos indica os $S$-processos privilegiados para obter as. DNEM's. Mostraremos que existem $v_{0}-2$ DNEM's. Como pela Observação 3.3 este é o número máximo de DNEM's, o algoritmo finaliza. Em seguida, apartir das ordens das'DNEM's obtidas, obteremos $\sharp(\Lambda \backslash \Gamma)$ simplesmente contando os pontos do diagrama de lacunas.

A existência das DNEM's amunciada será feita por indução sobre $i$. 
No primeiro passo do algoritmo para obter uma Base Standard de $\mathcal{O} d \mathcal{O}$ (Veja Algoritmo 2.2), o $S$-processo à ser considerado é

$$
S=x d y-\frac{v_{1}}{v_{0}} y d x
$$

onde $\psi(S)=\left(\lambda-v_{1}\right) t^{\lambda+v_{0}-1}$

Assim, definimos

$$
\Omega_{1}=\frac{1}{\lambda-v_{1}}\left(x d y-\frac{v_{1}}{v_{0}} d x\right)=\frac{1}{\lambda-v_{1}}\left(x \Omega_{0}-v_{1} y \Omega_{-1}\right)
$$

donde $\psi\left(\Omega_{1}\right)=t^{\lambda+v_{0}-1}$.

Se $\eta_{0}=3$, então não pode haver ontra DNEM.

Suponlia que $v_{0}>3$. Pela Proposição 3.3 , a próxima DNEM, se houver, será obtida através da redução final do $S$-processo de menor altura dentre

$$
y d y-v_{1} x^{j-1} \Omega_{1}
$$

e

$$
v_{0} y^{v_{0}-2} \Omega_{1}-v_{1} x^{v_{1}-j} d x .
$$

O primeiro tem altura $2 v_{1}-1$ e o segundo tem altura $\left(v_{0}-\dot{2}\right) v_{1}+\lambda+v_{0}-1$. Uma vez que $v_{0}>2$, temos que

$$
\begin{aligned}
&\left(v_{0}-2\right) v_{1}+\lambda+v_{0}-1=2 v_{1}+v_{0}-1+\left(v_{0}-2\right) v_{1}-j v_{0} \geq \\
& \geq 2 v_{1}+v_{0}-1+v_{1}-j v_{0}>2 v_{1}+v_{0}-1>2 v_{1}-1,
\end{aligned}
$$

e portanto, tomamos $\Omega_{2}=y d y-v_{1} x^{j-1} \Omega_{1}$, onde $\psi\left(\Omega_{2}\right)=\left(\lambda+v_{1}\right) t_{3}^{\lambda+v_{1}-1}+$ $\lambda t^{2 \lambda-1}$.

Se $v_{0}=4$, então não haverá mais DNEM.e o algoritmo finaliza, mostrando que as expressões para as DNEM's são válidas para $i=1$.

Suponlıa que o resultado seja válido para todo $k \operatorname{com} 1 \leq k \leq i<\left[\frac{v_{0}-1}{2}\right]$. Deste modo, devemos ter $v_{0}>2(i+1)-1 \mathrm{e}$

$$
\Omega_{2 k-1}=-\frac{1}{(k-2) \lambda+v_{1}} \cdot\left(x \Omega_{2(k-1)}-\left((k-1) \lambda+v_{1}\right) y \Omega_{2(k-1)-1}\right),
$$

onde $\psi\left(\Omega_{2 k-1}\right)=t^{k \lambda+v_{1}-1} \mathrm{e}$

$$
\Omega_{2 k}=y \Omega_{2(k-1)}-\left((k-1) \lambda+v_{1}\right) x \Omega_{2 k-1} ;,
$$


onde $\psi\left(\Omega_{2 k}\right)=\left(k \lambda+v_{1}\right) t^{k \lambda+v_{1}-1}+\lambda t^{(k+1) \lambda-1}$.

Se $v_{0}=2(i+1)$, então o algoritmo finaliza, uma vez que temos $2 i$ DNEM's. Vamos supor que $v_{0}>2(i+1)$.

Segundo a Proposição 3.3, para obter a próxima DNEM, se houver, basta considerar dentre os $S$-processos

$$
S_{1}=x \Omega_{2 i}-\left(i \lambda+v_{1}\right) y \Omega_{2 i-1}
$$

e

$$
S_{2}=y^{v_{0}-(2 i+1)} \Omega_{2 i}-\left(i \lambda+v_{1}\right) x^{v_{1}-i j-1} \Omega_{-1},
$$

o de menor altura.

Como

$$
\begin{aligned}
v\left(h t\left(S_{2}\right)\right) & =\left(v_{0}-(2 i+1)\right) v_{1}+i \lambda+v_{1}-1>v_{1}+i \lambda+v_{1}-1> \\
& >v_{0}+i \lambda+v_{1}-1=v\left(h t\left(S_{1}\right)\right),
\end{aligned}
$$

tomamos

$$
\Omega_{2(i+1)-1}=\frac{-1}{(i-1) \lambda+v_{1}}\left(x \Omega_{2 i}-\left(i \lambda+v_{1}\right) y \Omega_{2 i-1}\right),
$$

onde $\psi\left(\Omega_{2(i+1)-1}\right)=t^{(i+1) \lambda+v_{0}-1}$.

Se $v_{0}=2(i+1)+1$, então o algoritmo finaliza, pois teremos $v_{0}-2=$ $2(i+1)-1$ DNEM's.

Se $v_{0}>2(i+1)+1$ então, novamente pela Proposição 3.3 , o próximo $S$-processo a ser analisado é o de menor altura dentre

$$
S_{1}=y \Omega_{2 i}-\left(i \lambda+v_{1}\right) x^{j-1} \Omega_{2(i+1)-1}
$$

e

$$
S_{2}=y^{v_{0}-2(i+1)} \Omega_{2(i+1)-1}-x^{v_{1}-(i+1) j} \Omega_{-1} .
$$

Uma vez que $v\left(h t\left(S_{1}\right)\right)=(i+1) \lambda+v_{1}+v_{0}-1$ e $v\left(h t\left(S_{2}\right)\right)=\left(v_{0}-2(i+\right.$ 1)) $v_{1}+(i+1) \lambda+v_{0}-1>v\left(h t\left(S_{1}\right)\right)$, tomamos

$$
\Omega_{2(i+1)}=y \Omega_{2 i}-\left(i \lambda+v_{1}\right) x^{j-1} \Omega_{2(i+1)-1},
$$

onde $\psi\left(\Omega_{2(i+1)}\right)=\left((i+1) \lambda+v_{1}\right) t^{(i+1) \lambda+v_{1}-1}+\lambda t^{(i+2) \lambda-1}$.

Assim, as expressões para as DNEM's, são verdadeiras para todo $1 \leq$ $i \leq\left[\frac{v_{0}-1}{2}\right]$. Resta agora efotinarmos a contagem dos pontos do diagrama de lacunas.

Para facilitar, vamos considerar dois casos:

Caso a) $v_{0}$ par. Neste caso, o diagrama de lacunas é 


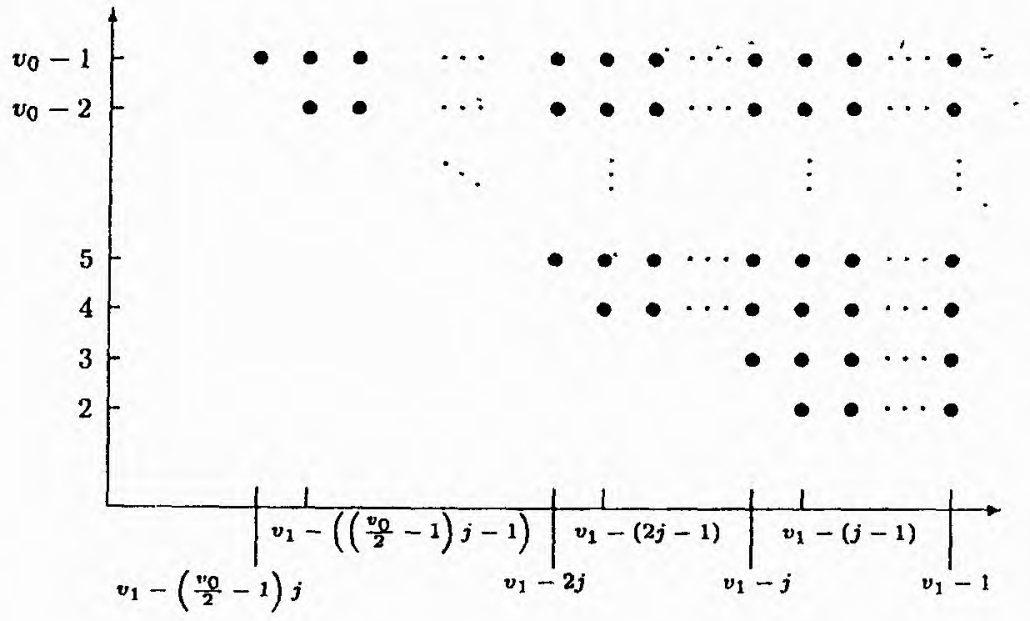

Assim,

$$
\begin{aligned}
H(\Lambda \backslash \Gamma) & =\sum_{i=2}^{v_{0}-1}\left(v_{0}-i\right)+\sum_{i=1}^{\frac{v_{0}-2}{2}}(j-2)\left(v_{0}-2 i\right)= \\
& =\frac{\left(v_{0}-1\right)\left(v_{0}-2\right)}{2}+(j-2)\left(\frac{v_{0}}{2}\right)\left(\frac{v_{0}-2}{2}\right)= \\
& =\left(\frac{v_{0}-2}{2}\right)\left((j-2) \cdot \frac{v_{0}}{2}+v_{0}-1\right)= \\
& =\left[\frac{v_{0}-1}{2}\right]\left((j-2)\left[\frac{v_{0}}{2}\right]+2\left[\frac{v_{0}}{2}\right]-1\right)=\left[\frac{v_{0}-1}{2}\right]\left(j\left[\frac{v_{0}}{2}\right]-1\right) .
\end{aligned}
$$

Caso b) $v_{0}$ ímpar. O diagrama de lacunas para este caso é

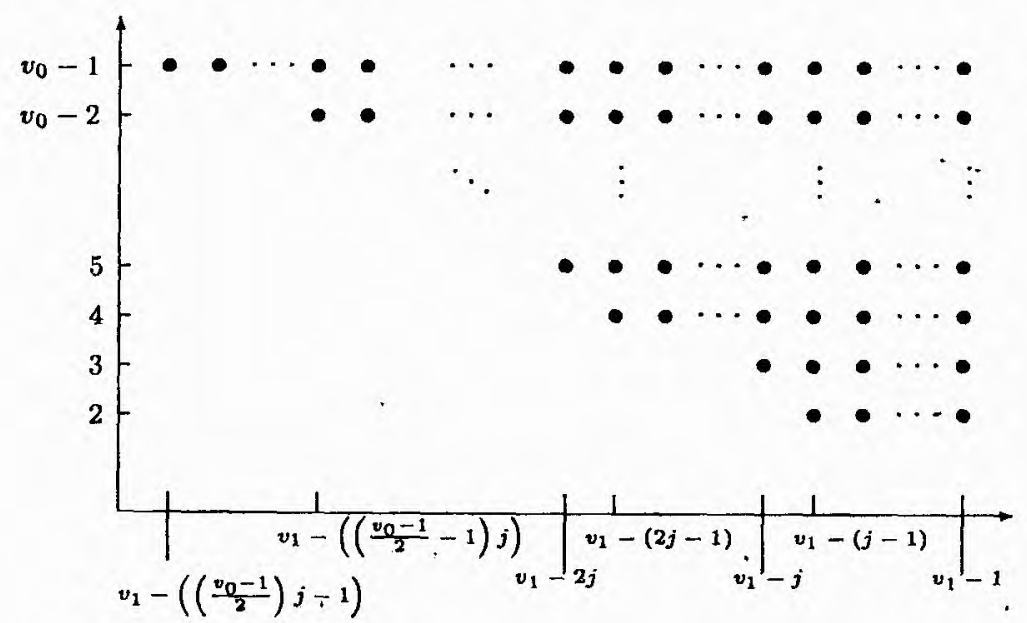


Dessa forma, temos .

$$
\begin{aligned}
\sharp(\Lambda \backslash \Gamma) & =\sum_{i=2}^{v_{0}-1}\left(v_{0}-i\right)+\sum_{i=1}^{\frac{v_{0}-1}{2}}(j-2)\left(v_{0}-2 i\right)= \\
& =\frac{\left(v_{0}-1\right)\left(v_{0}-2\right)}{2}+(j-2)\left(\frac{v_{0}-1}{2}\right)\left(\frac{v_{0}-1}{2}\right)= \\
& =\left(\frac{v_{0}-1}{2}\right)\left((j-2) \frac{v_{0}-1}{2}+v_{0}-2\right)= \\
& =\left[\frac{v_{0}-1}{2}\right]\left((j-2)\left[\frac{v_{0}}{2}\right]+2\left[\frac{v_{0}}{2}\right]-1\right)=\left[\frac{v_{0}-1}{2}\right]\left(j\left[\frac{v_{0}}{2}\right]-1\right) .
\end{aligned}
$$

As proposições anteriores e a análise de alguns exemplos nos levam a formular a seguinte conjectura.

Conjectura 5.1 Dado o ramo $\lambda$-canônico

$$
C:\left\{\begin{array}{l}
x=t^{v_{0}} \\
y=t^{v_{1}}+t^{\lambda},
\end{array}\right.
$$

o conjunto $\Lambda \backslash \Gamma$ das lacunas especiais, pode ser obtido aplicando o seguinte. algoritmo:

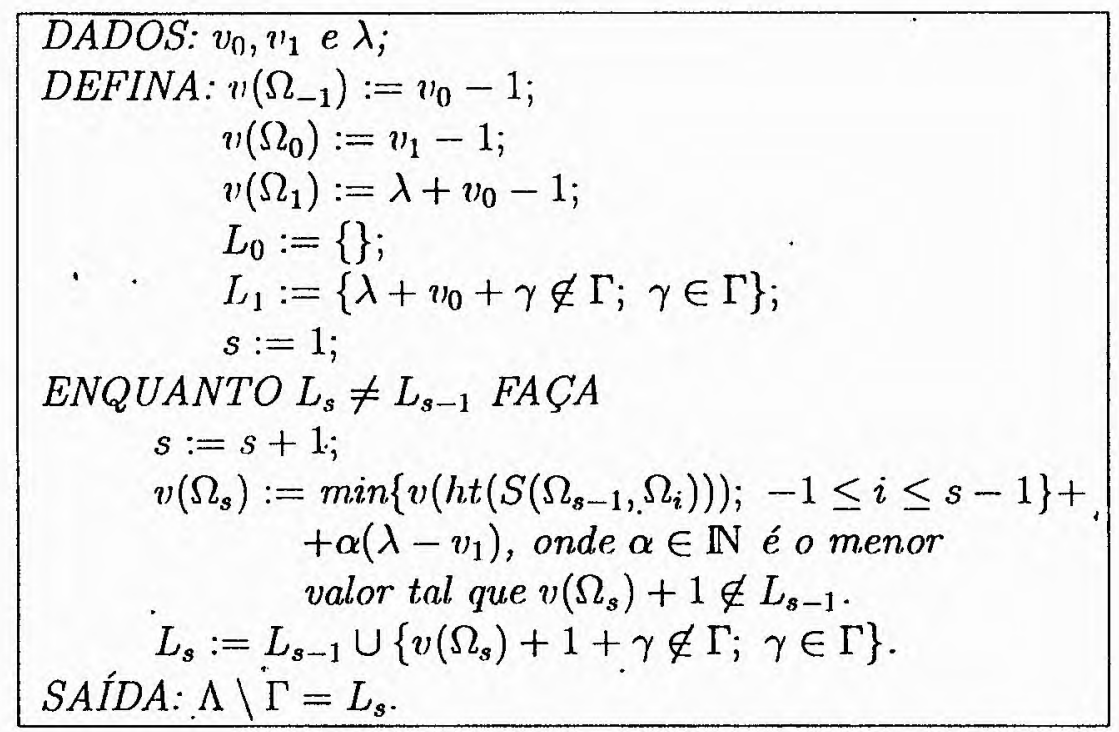


Exemplo 5.5 a) Considerando $\Gamma=\left\langle 5, v_{1}\right\rangle$, com $5<v_{1} \leq 11$, vemos qur o Exemplo 5.3 e o estudo completo do conjunto de lacunas especiais para $o$ semigrupo $\Gamma=\langle 5,11\rangle$, apresentado no Exemplo 5.4, confirmam a conjectura para qualquer $\lambda$.

b) Seja $\Gamma=\langle 6,11\rangle$. O conjunto das lacunas que podem ser tomadas como invariante de Zariski é $\{13,14,15,19,20,21,25,26,31,32,37,43\}$.

O Corolário 5.1 comprova a conjectura para

$$
\lambda \in\{14,20,25,26,31,32,37,43\} .
$$

A Proposição 5.2, por sua vez, também mostra a veracidade da conjectura para $\lambda=\{13,19\}$. Resta, portanto analisar $\lambda \in\{15,21\}$.

Se $\lambda=15$, ent.ão $\lambda-v_{1}=4$ e o algortimo que nos dá uma Basc Standard Mínima para $\mathcal{O} d \mathcal{O}$, Algoritmo 2.2, fornece as seguintes DNEM's:

$$
\Omega_{1}=x d y-\frac{11}{6} y d x \operatorname{com} \psi\left(\Omega_{1}\right)=4 t^{20}
$$

$e$

$$
\Omega_{2}=y^{2} d y-\frac{11}{4} x^{2} \Omega_{1} \operatorname{com} \psi\left(\Omega_{2}\right)=37 t^{36}+51 t^{40}+15 t^{44} .
$$

Assim, $\Lambda \backslash \Gamma=\{21,27,32,37,38,43,49\}$, como indica a conjectura.

Se $\lambda=21$, ent.äo $\lambda-11=10$ e temos $\Omega_{1}=x d y-\frac{11}{6} y d x \operatorname{com} . \psi\left(\Omega_{1}\right)=$ $10 t^{26}$. Como $v\left(y \Omega_{1}\right)+1=38$ e $v\left(y^{2} \Omega_{1}\right)+1=49$, temos que $l=43$ é a lacuna limitante.

Continuando com o Algoritmo 2.2, temos

$$
\Omega_{2}=y^{2} d x-\frac{11}{10} x \Omega_{1}
$$

com. $\psi\left(\Omega_{2}\right)=43 t^{42}+53 t^{52}+21 t^{62}$. Assim, a lacuna limitante passa a ser $l=37$ o que indica o fim do algoritmo e a confirmação da conjectura para este semiyrupo.

\subsection{Diagramas Admissiveis}

Laudal e Pfister, em [LaP], mostram que para curvas algebróides irredutíveis planas, todos os valores do número $\tau$ de Tjurina entre o mínimo, que corresponde ao caso genérico, e o máximo, que sabemos sẹr $\mu$, são atingidos. 
Ou seja, existe' uma curva com semigrupo $\Gamma=\left\langle v_{0}, v_{1}\right\rangle$, de forma que seu $o$ número de Tjurina seja $\tau$.

Já mencionamos anteriormente, e os Exemplos 5.2 e 5.4 comprovam o fato que, fixado o semigrupo $\Gamma=\left\langle v_{0}, v_{1}\right\rangle$, podemos ter curvas com mesmo número de Tjurina, mas com conjuntos de lacunas especiais distintos.

Desse modo, algumas questões surgem, como por exemplo:

Sejam $\mathcal{L}=\left\{l \notin \Gamma ; l>v_{0}+v_{1}\right\}$ e $\mathcal{L}_{0}$ um subconjunto de $\mathcal{L}$. Como saber se $\mathcal{L}_{0}$ é o conjunto de lacunas especiais de um ramo com semigrupo $\Gamma=\left\langle v_{0}, v_{1}\right\rangle$, ou mais geralmente, quais são todos os possíveis coujuntos de lacunas especiais para um ramo com semigrupo $\Gamma=\left\langle v_{0}, v_{1}\right\rangle$ fixo?

É claro que dado um semigrupo $\Gamma$, não necessariamente de gênero 1 , podemos realizar, através das técuicas apresentadas, o estudo completo de $\Lambda \backslash \Gamma$ para esta classe de equisingularidade e assim responder às questões acima para $\Gamma$, como fizemos nos Exemplos 3.5 e 5.4 .

Nesta seção, mostraremos que determinar todos os possíveis conjuntos de lacunas especiais, ou equivalentemente, todos os diagramas de lacunas para os ramos com semigrupo $\Gamma=\left\langle v_{0}, v_{1}\right\rangle$ é uma questão puramente combiuatória e fácil de ser respondida utilizando um algoritmo que apresentaremos adiante.

Seja dado um ramo com semigrupo $\Gamma$,

$$
C:\left\{\begin{array}{l}
x=t^{v_{0}} \\
y=\sum_{l \geq v_{1}} a_{l} t^{i},
\end{array}\right.
$$

onde $a_{v_{1}}=1$ e $a_{l} \in K$ para todo $l$.

Desse modo, se $\varphi$ e $\psi$ são os homomorfismos de $\mathcal{O}$-módulos dados, respectivamente em (2.1) e (2.2), então

$$
\psi(d x)=v_{0} t^{v_{0}-1} \quad \text { e } \quad \psi(d y)=\sum_{l \geq v_{1}} l a_{l} t^{l-1}
$$

Ágora observe que em

$$
\varphi\left(x^{\alpha} y^{\beta}\right)=t^{\alpha v_{0}}\left(\sum_{l \geq v_{1}} a_{l} t^{l}\right)^{\beta}
$$

o termo de menor ordem em $t$ no qual $a_{j}$ ocorre é clarameute da forma

$$
\left(P\left(a_{i} ; v_{1}<i<j\right)+\beta a_{j}\right) t^{\alpha v_{0}+(\beta-1) v_{1}+j},
$$

onde $P\left(a_{i} ; v_{1}<i<j\right)^{\prime}$ é um polinômio com “coeficientes em $K$ nas indeterminadas $a_{i}$ para $v_{1}<i<j$. 
Do mesmo modo, o termo de menor ordem em $t$ em que $a_{j}$ ocorre em $\psi\left(x^{\alpha} y^{\beta} d x\right)$, respectivannente em $\psi\left(x^{\alpha} y^{\beta} d y\right)$ é

$$
\left(P\left(a_{i} ; v_{1}<i<j\right)+v_{0} \beta a_{j}\right) t^{\alpha v_{0}+(\beta-1) v_{1}+j+v_{0}-1},
$$

respectivamente da forma

$$
\left(Q\left(a_{i} ; v_{1}<i<j\right)+\left(\beta v_{1}+j\right) a_{j}\right) t^{\alpha v_{0}+(\beta-1) v_{1}+j+v_{1}-1},
$$

onde $Q\left(a_{i} ; v_{1}<i<j\right)$ é um polinômio com coeficientes em $K$ nas indeterminadas $a_{i}$, com $v_{1}<i<j$.

De modo mais geral, temos o fato a seguir.

Observação 5.4 Sejam $\omega=\sum_{i \in \mathbb{N}^{*}} b_{i} t^{i} \in K[[t]], x$ e y como na parametrização de C. Então, o termo de menor ordem em $t$ em $\varphi\left(x^{\alpha} y^{\beta}\right) \omega$ em que $b_{j}$ ocorre é da forma

$$
\left(R\left(b_{i} ; 1 \leq i<j\right)+b_{j}\right) t^{\alpha v_{0}+\beta v_{1}+j},
$$

onde $R\left(b_{i} ; 1 \leq i<j\right)$ é um polinômio com coeficientes em $K$ nas indeterminadas $b_{i}$ com $1 \leq i<j$.

Como estamos nos restringindo a ramos $C$ de gênero 1 , temos que $F=$ $\{x, y\}$ é uma Base Standard Mínima para o anel local $\mathcal{O}$ de $C$.

Sejam $\alpha, \beta \in \mathbb{N}^{2}$, tais que $v\left(F^{\alpha}\right)<v\left(F^{\beta}\right)$ e $\omega=\sum_{i \in \mathbb{N}^{*}} b_{i} t^{i} \in K[[t]]$. O termo de menor ordem em $t$ em que $b_{j}$ ocoire em $\varphi\left(c_{\alpha} F^{\alpha}+c_{\beta} F^{\beta}\right) \omega$, oude $c_{\alpha}, c_{\beta} \in K^{*}$, é dado pelo termo de menor ordem em que $b_{j}$ ocorre em $c_{\alpha} F^{\alpha} \omega$, onde ao seu coeficiente é somado uma expressão polinomial em $b_{i}$, com $1 \leq i<j$.

Vamos supor que as DNEM's foram obtidas por uma redução final dos $S$-processos, como descritos ua Proposição 3.3 ; ou seja, se $B=\left\{\Omega_{-1}, \Omega_{0}\right.$, $\left.\Omega_{1}, \ldots, \Omega_{r}\right\}$ é uma Base Standard Mínima para $\mathcal{O} d \mathcal{O}$, com $v\left(\Omega_{i}\right)<v\left(\Omega_{i+1}\right)$, para $i=-1, \ldots, r-1, \Omega_{-1}=d x$ e $\Omega_{0}=d y$, então

$$
\Omega_{i+1}=\sum_{-1 \leq j \leq i} g_{j} \Omega_{j},
$$

para $i=0, \ldots, r-1, g_{j} \in \mathcal{O}$ e $v\left(g_{i} \Omega_{i}\right)=v\left(g_{k} \Omega_{k}\right)<v\left(g_{j} \Omega_{j}\right)$, para algum $k<i$ e todo $j \neq k$ com $j<i$.

Vejamos que informações podemos obter a respeito do. coeficiente de $t^{\eta}$ em $\Omega_{i+1}$. 

forma

Inicialmente, note que o coeficiente de $t^{\eta}$ de $g_{j} \Omega_{j}$, com $j=-1, \ldots, i$ é da

$$
P_{j}\left(b_{j k} ; v\left(\Omega_{j}\right) \leq k \leq l_{j}-1\right)+c b_{j l_{j}},
$$

onde $b_{j k}$ são os cóficientes de $t^{k}$ em $\Omega_{j}, c \in K^{*} \mathrm{e} l_{j}=\eta-v\left(g_{j}\right)$. Além disso, escrevendo $g_{j}=\sum_{\alpha \in A} d_{\alpha} F^{\alpha}$ com a amplitude desta representação igual a $1 \mathrm{e}$ $v\left(g_{j}\right)=v\left(d_{\alpha_{0}} F^{\alpha_{0}}\right)$, temos que a parcela $c b_{j l_{j}}$ em (5.6) provém de $d_{\alpha_{0}} F^{\alpha_{0}} \Omega_{j}$.

Como na representação de $\Omega_{i+1}$ dada acima, temos para $-1 \leq j<i$,

$$
v\left(g_{i}\right)+v\left(\Omega_{j}\right)<v\left(g_{i}\right)+v\left(\Omega_{i}\right) \leq v\left(g_{j}\right)+v\left(\Omega_{j}\right),
$$

ou seja, $v\left(g_{i}\right)<v\left(g_{j}\right)$, para todo $-1 \leq j<i$.

Assim, $l_{i}=\eta-v\left(g_{i}\right)>\eta-v\left(g_{j}\right)=l_{j}$, para todo $-1 \leq j<i$, on em outras palavras, o coeficiente de $t^{\eta} \mathrm{em} \Omega_{i+1}$, que é a soma dos coeficientes de $t^{\eta}$ em $g_{j} \Omega_{j}$ para $-1 \leq j \leq i$, é da forma

$$
P_{i+1}\left(b_{j k} ; v\left(\Omega_{j}\right) \leq k \leq l_{j}-1 ; k \neq l_{i} ;-1 \leq j \leq i\right)+c^{\prime} b_{i l_{i}},
$$

onde $c^{\prime} \in K^{*}, P_{i+1}^{*}\left(b_{j k} ; v\left(\Omega_{j}\right) \leq k \leq l_{j}-1 ; k \neq l_{i}-1 \leq j \leq i\right)$ é $\mathrm{nm}$ polinômio nos $b_{j k}$ 's.

O lema abaixo, nos fornece informações mais explícitas a respeito do coeficiente de $t^{\eta}$ em $\Omega_{i+1}$.

Lema 5.3 Sejam C um ramo de gên.ero 1, dado por

$$
\left\{\begin{array}{l}
x=t^{v_{0}} \\
y=t^{v_{1}}+\sum_{j>v_{1}} a_{j} t^{j}
\end{array}\right.
$$

e $\Omega_{i+1}$ com $i=0, \ldots, r-1$, como descritas na Proposição 3.3. Então o coeficiente de t. $t^{\eta}$ em $\Omega_{i+1}$ é da forma

$$
Q_{i+1}^{\eta}\left(a_{k} ; v_{1}<k<l\right)+c_{\eta, i+1} a_{l},
$$

com $c_{\eta, i+1} \in K^{*}, l=\eta+1-\sum_{s=0}^{i} \theta_{s}$, onde $\theta_{s}=v\left(h t\left(\Omega_{s+1}\right)\right)-v\left(\Omega_{s}\right)$ para $s=0, \ldots, r$ e $Q_{i+1}^{\eta}\left(a_{k} ; v_{1}<k<l\right)$ um polinômio com coeficientes em. $K$ nas indeterminadas $a_{k}$, para $v_{1}<k<l$.

Dem.: Faremos a demonstração por indução sobre $i$.

$$
\text { Para } i=0 \text {, temos } \Omega_{1}=\left(-\frac{v_{1}}{v_{0}} y+\cdots\right) d x+(x+\cdots) d y=g_{-1} \Omega_{-1}+g_{0} \Omega_{0} \text {. }
$$


De (5.4) e (5.5) temos que os coeficientes de $t^{\eta}$ em $g_{-1} \Omega_{-1}$ è em $g_{0} \Omega_{0}$ são respectivamente

$$
Q_{-1}^{\eta}\left(a_{k} ; v_{1}<k<\eta-v_{0}\right)+c a_{\eta-v_{0}+1}
$$

e

$$
Q_{0}^{\eta}\left(a_{k} ; v_{1}<k<\eta-v_{0}\right)+d a_{\eta-v_{0}+1}
$$

onde $c=-v_{1}$ e $d=\eta-v_{0}+1$. Assim, o coeficiente de $t^{\eta}$ em $\Omega_{1}$ é

$$
Q_{1}^{\eta}\left(a_{k} ; v_{1}<k \leq \eta-v_{0}\right)+(c+d) a_{\eta-v_{0}+1}
$$

Como $c+d=\eta+1-v_{0}-v_{1} \in K^{*}$, pois $v\left(\Omega_{1}\right)+1>v_{0}+v_{1}$ e $\theta_{0}=$ $v\left(h t\left(\Omega_{1}\right)\right)-v\left(\Omega_{0}\right)=v_{0}$, o lema é verdadeiro para $i=0$.

Vamos supor que o lema seja verdadeiro para todo $j$ com $-1 \leq j \leq i<r$, ou seja, o coeficiente de $t^{\eta}$ em $\Omega_{j}$ seja da forma

$$
Q_{j}^{\eta}\left(a_{k} ; v_{1}<k \leq \eta-\sum_{s=0}^{j} \theta_{s}\right)+c_{\eta, j} a_{\eta+1-\sum_{s=0}^{j} \theta_{s}},
$$

$\operatorname{com} c_{\eta, j} \in K^{*}$.

Pela Proposição 3.3, podemos considerar

$$
\Omega_{i+1}=\sum_{k=-1}^{i} g_{k} \Omega_{k}
$$

$\operatorname{com} v\left(g_{i} \Omega_{i}\right)=\min _{-1 \leq k \leq i}\left\{v\left(g_{k} \Omega_{k}\right)\right\}$. O coeficiente de $t^{\eta}$ em $\Omega_{i+1}$ é a soma dos coeficientes de $t^{\eta}$ em cada $g_{k} \Omega_{k}$, para $k=-1, \ldots, i$. O maior índice para os parâmetros a's que ocorrem nos coeficientes de $g_{k} \Omega_{k}$ é por (5.4), (5.5) e por (5.6), respectivamente

$$
\begin{array}{ll}
k=-1: & \eta+1-v\left(g_{-1}\right)+v_{1}-v_{0} \\
k=0: & \eta+1-v\left(g_{0}\right) \\
k>0: & \eta+1-v\left(g_{k}\right)-\sum_{s=0}^{k-1} \theta_{s} .
\end{array}
$$

Observe que

$$
\begin{gathered}
v\left(\Omega_{1}\right)>\theta_{0}+v\left(\Omega_{0}\right) \\
v\left(\Omega_{2}\right)>\theta_{1}+v\left(\Omega_{1}\right), \\
\vdots \\
v\left(\Omega_{j+1}\right)>\theta_{j}+v\left(\Omega_{j}\right),
\end{gathered}
$$


para $j=0, \ldots, r-1$. Assim, $v\left(\Omega_{j+1}\right)>v\left(\Omega_{l}\right)+\sum_{k=s}^{j} \theta_{s}$ e temos que

$$
v\left(g_{i}\right)+v\left(\Omega_{j}\right)+\sum_{k=s}^{i-1} \theta_{s}<v\left(g_{i}\right)+v\left(\Omega_{i}\right) \leq v\left(g_{j}\right)+v\left(\Omega_{j}\right)
$$

para $1 \leq j<i$, ou seja,

$$
v\left(g_{i}\right)+\sum_{s=j}^{i-1} \theta_{s}<v\left(g_{j}\right)
$$

Portanto, temos que

$$
v\left(g_{i}\right)+\sum_{s=0}^{i-1} \theta_{s}<v\left(g_{j}\right)+\sum_{s=0}^{j-1} \theta_{s}
$$

para $j=1, \ldots, i-1$.

Além disso, somando as desigualdades em (5.7), obtemos

$v\left(g_{i}\right)+v\left(\Omega_{0}\right)+\sum_{s=0}^{i} \theta_{s}<v\left(g_{i}\right)+v\left(\Omega_{i}\right) \leq \min \left\{v\left(g_{-1}\right)+v\left(\Omega_{-1}\right), v\left(g_{0}\right)+v\left(\Omega_{0}\right)\right\}$ isto é,

$$
v\left(g_{i}\right)+\sum_{s=0}^{i} \theta_{s}<\min \left\{v\left(g_{-1}\right)+v_{0}-v_{1}, v\left(g_{0}\right)\right\}
$$

Dessa forma, o maior dos índices anteriormente mencionados, é $\eta+1$ $v\left(g_{i}\right)-\sum_{s=0}^{i-1} \theta_{s}=\eta+1-\sum_{s=0}^{i} \theta_{s}$ e o coeficiente de $t^{\eta}$ em $\Omega_{i+1}$ é da forma

$$
Q_{i+1}^{\eta}\left(a_{k} ; \dot{v_{1}}<k<\eta-\sum_{s=0}^{i} \theta_{s}\right)+c_{\eta, i+1} a_{\eta+1-\sum_{s=0}^{i} \theta_{s}}
$$

$\operatorname{com} c_{\eta, i+1} \in K^{*}$

Observe que o lema anterior garante que uma DNEM, obtida como descrito na Proposição 3.3 , pode ter qualquer ordem acima da ordem da altıra do $S$-processo que a origina. De fato, como o coeficiente de $t^{\eta}$ em $\Omega_{i}$ é da forma

$$
Q_{i}^{\eta}\left(a_{k} ; v_{1}<k<\eta-\sum_{s=0}^{i-1} \theta_{s}\right)+c_{\eta, i} a_{\eta+1-\sum_{s=0}^{i-1} \theta_{s}}
$$


$\operatorname{com} c_{\eta, i} \in K^{*}$. Para que $\Omega_{i}$ tenha ordem $\nu>v\left(h t\left(S_{i}\right)\right)$, onde $S_{i}$ é o $S$ processo que origina $\Omega_{i}$, dado na Proposição 3.3 , basta tomarmos

$$
a_{\eta+1-\sum_{s=0}^{i-1} \theta_{s}}=c_{\eta, i}^{-1} Q_{i}^{\eta}\left(a_{k} ; v_{1}<k<\eta-\sum_{s=0}^{i-1} \theta_{s}\right),
$$

para $v\left(h t\left(S_{i}\right)\right)<\eta<\nu \mathrm{e}$

$$
a_{\nu+1-\sum_{s=0}^{i-1} \theta_{s}} \neq c_{\nu, i}^{-1} Q_{i}^{\nu}\left(a_{k} ; v_{1}<k<\nu-\sum_{s=0}^{i-1} \theta_{s}\right) .
$$

Note que, os parâmetros a's fixados para termos $v\left(\Omega_{i}\right)=\nu$ não serão relevantes para que $\Omega_{i+1}$ tenha uma determinada ordem, pois

$$
v\left(h t\left(S_{i}\right)\right)<\eta<\nu=v\left(\Omega_{i}\right)<v\left(h t\left(S_{i+1}\right)\right)<v\left(\Omega_{i+1}\right),
$$

e assim os parâmetros $a$ 's sobre os quais impomos condições têm íudice maior que

$$
v\left(h t\left(S_{i+1}\right)\right)+1-\sum_{s=0}^{i} \theta_{s}=v\left(\Omega_{i}\right)+1-\sum_{s=0}^{i-1} \theta_{s}=\nu+1-\sum_{s=0}^{i-1} \theta_{s} .
$$

Portanto, segue diretamente dos fatos anteriores, o seguinte teorema.

Teorema 5.2 Seja $\Gamma=\left\langle v_{0}, v_{1}\right\rangle$. Um. subconjunto $\mathcal{L}_{0}$ não vazio de $\mathcal{L}=$ $\left\{l \notin \Gamma ; l>v_{0}+v_{1}\right\}$ é um conjunto de lacunas especiais para um ramo com. semigrupo $\Gamma$ se, e somente se, $\mathcal{L}_{0}$ é obtido pelo seguinte algoritmo: 


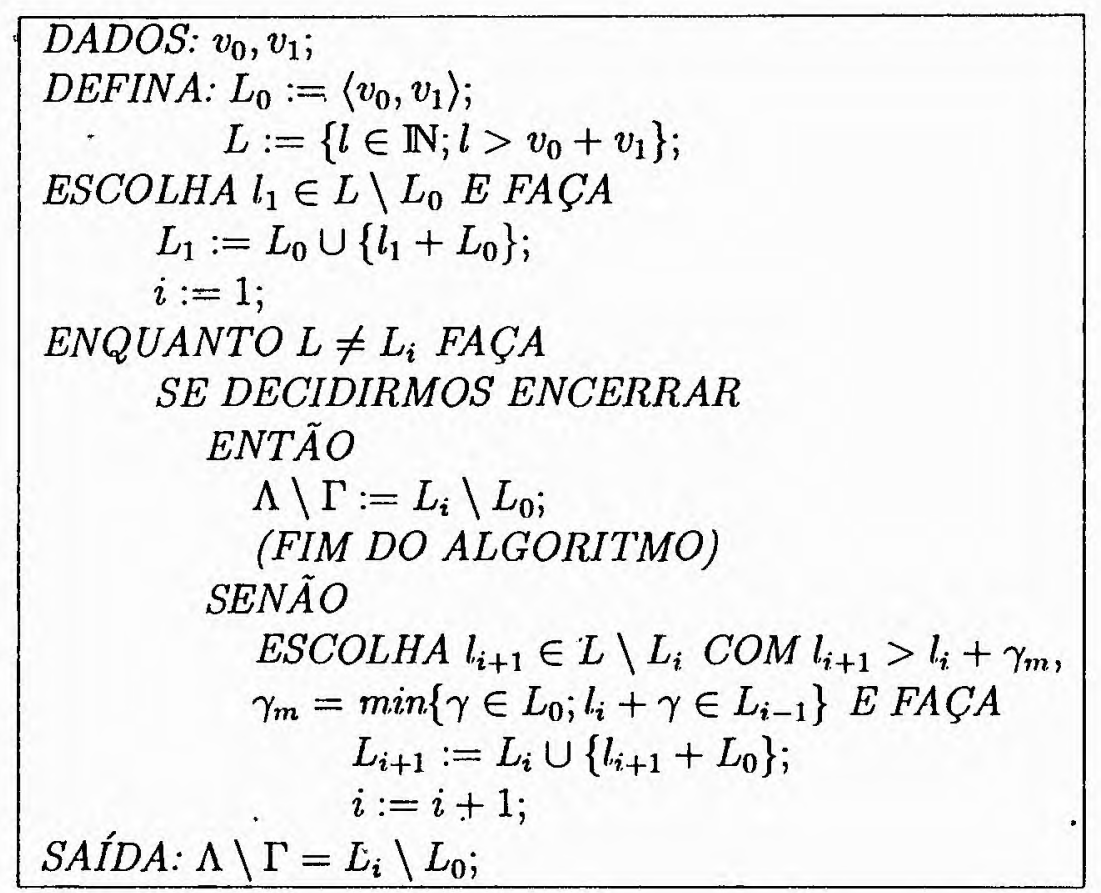

Dem.: Segue diretamente dos fatos apresentados anteriormente, observando que a escolha de $l_{1}$ corresponde à escollha de $v\left(\Omega_{1}\right)+1, l_{i}+\gamma_{m}$ corresponde à ordem da altura do $S$-processo indicado na Proposição 3.3 e $l_{i+1}$ corresponde 'à escolha de $v\left(\Omega_{i+1}\right)+1$.

Exemplo 5.6 Seja $\Gamma=\langle 6,13\rangle$. O conjunto das lacunas de $\Gamma$ que são maiores que $v_{0}+v_{1}=19$, ou seja, que podem ser ordens de DNEM's, é

$$
\mathcal{L}=\{20,21,22,23,27,28,29,33,34,35,40,41,46,47,53,59\} .
$$

$\odot$ que dizer dos subconjuntos.

$$
\mathcal{L}_{1}=\{20,27,33,34,40,41,46,47,53,59\}
$$

$e$

$$
\mathcal{L}_{2}=\{20,29,33,35,40,41,46,47,53,59\}
$$

de $\mathcal{L}$, quanto a possibilidade de serem conjuntos de lacunas especiais de um. ramo com semigrupo $\Gamma$. 
Como $\min \left\{\mathcal{L}_{1}\right\}=\min \left\{\mathcal{L}_{2}\right\}=20$, o algoritmo do teorema anterior, nos diz que $v\left(\Omega_{1}\right)+1=20$. Desta forma, $\left\{v\left(\Omega_{1}\right)+1+\gamma \notin \Gamma ; \gamma \in \Gamma\right\}=$ $\{20,33,46,59\}$ é subconjunto do conjunto das lacunas especiais. Uma vez que tal conjunto está contido em $\mathcal{L}_{1}$ e em. $\mathcal{L}_{2}$, não podemos concluir nada até este ponto.

Continuando com o algoritmo anterior, vamos escolher $l \in \mathcal{L} \backslash \mathcal{L}_{i}$ para $i=1,2$. Assim, vamos proceder a análise separadamente.

Análise de $\mathcal{L}_{1}$.

Se houver uma $D N E M \Omega_{2}$, além de $\Omega_{1}$, devemos ter $v\left(\Omega_{2}\right)+1>20+6=$ 26, pois $6=\min \{\gamma \in \Gamma ; 20+\gamma \in \Gamma\}$. Desse modo, devemos admitir que $v\left(\Omega_{2}\right)+1=27$ e $\left\{v\left(\Omega_{2}\right)+1+\gamma \notin \Gamma ; \gamma \in \Gamma\right\}=\{27,33,40,46,53,59\}$ está contido em $\Lambda \backslash \Gamma$. Uma vez que esse conjunto está contido em. $\mathcal{L}_{1}$, nenhuma conclusão pode ser tirada.

Havendo uma nova DNEM $\Omega_{3}$, esta deve ser de modo que $v\left(\Omega_{3}\right)+1>$ $27+6=33$. Assim, vamos admitir que $v\left(\Omega_{3}\right)+1=34$, o que implica que $\left\{v\left(\Omega_{3}\right)+1+\gamma \notin \Gamma ; \gamma \in \Gamma\right\}=\{34,40,46,47,53,59\}$ está contido em. $\Lambda \backslash \Gamma$. Novamente como todos estes elementos pertencem $\dot{a} \mathcal{L}_{1}$, nada podemos afirmar.

Para que tenhamos uma DNEM $\Omega_{4}$, obrigatoriamente devemos ter $v\left(\Omega_{4}\right)+1>34+6=40$. Conseqüentem.ente, devemos ter $v\left(\Omega_{4}\right)+1=41 \mathrm{e}$ $\left\{v\left(\Omega_{4}\right)+1+\gamma \notin \Gamma ; \gamma \in \Gamma\right\}=\{41,47,53,59\}$.

Portanto, $\mathcal{L}_{1}$ é o conjunto de lacunas especiais para algum ramo com. semigrupo $\Gamma=\langle 6,13\rangle$. A saber, o Teorema 5.1 indica que genericamente temos $\Lambda \backslash \Gamma=\mathcal{L}_{1}$.

Análise de $\mathcal{L}_{2}$.

Para que ocorra uma DNEM $\Omega_{2}$, devemos ter $v\left(\Omega_{2}\right)+1>20+6$, o que nos leva a concluir que $v\left(\Omega_{2}\right)+1=29$ e que $\left\{v\left(\Omega_{2}\right)+1+\gamma \notin \Gamma ; \gamma \in \Gamma\right\}=$ $\{29,35,41,47,53,59\}$ são lacunas especiais.

Se houvesse uma outra DNEM $\Omega_{3}$, teríamos $v\left(\Omega_{3}\right)+1>29+13=42$, no entanto em $\mathcal{L}_{2}$ temos o valor 40 . Portanto, $\mathcal{L}_{2} \backslash\{40\}$ é o conjunto de lacunas especiais para um ramo com semigrupo $\Gamma=\langle 6,13\rangle$, mas $\mathcal{L}_{2}$ não. $A$ saber. os ramos da forma

$$
C:\left\{\begin{array}{l}
x=t^{6} \\
y=t^{13}+t^{14}+\frac{27}{26} t^{15}+\frac{560}{507} t^{16}+a t^{17}+\cdots,
\end{array}\right.
$$

com $a \neq \frac{63017}{1014}$, admitem $\Lambda \backslash \Gamma=\mathcal{L}_{2} \backslash\{40\}$, obtidos aplicando o Algoritmo 2.2 e impondo condições sobre os parâmetros. 


\section{Capítulo 6}

\section{Ramos de Multiplicidade Baixa}

Ebey, no seu artigo [E] de 1967, apresenta uuma tabela, a classificação, com respeito à relação de equivalência definida no Capítulo 3 , dos ramos com semigrupo $\Gamma=\left\langle v_{0}, v_{1}, \ldots, v_{g}\right\rangle$, onde $v_{0}=2, v_{0}=3$ e no caso de $v_{0}=$ 4 considera $v_{1} \leq 11$. Bruce e Gaffuey, em [BGa] de 1982, usaudo ontros métodos, obtém parte dos resultados de Ebey. Carbonne, em [Car] de 1998, retoma a tabela de Ebey, acrescentando o valor do número de Tjurina $\tau$ para cada classe de equivalência. No entanto, algums erros tipográficos de $[\mathrm{E}]$ são reproduzidos e o caso do semigrupo $\Gamma=\langle 4,11\rangle$ contém um pequeno erro.

Neste capítulo, além de reapresent,ar a tabela mencionada, com as devidas correções, substituímos a informação do número de Tjurina $\tau$, pelo conjunto das lacunas especiais $\Lambda \backslash \Gamma$ que, como já mencionamos no Capítulo 3 , é um invariante mais fino que permite obter $\tau$ por meio da relação $\tau=\mu-\sharp(\Lambda \backslash \Gamma)$.

Para dar uma pequena indicação da efetividade dos nossos métodos, estenderemos a tabela de classificação para todos os ramos com $v_{0} \leq 4$ e no caso de $v_{0}=5$ consideramos $v_{1} \leq 11$. O valor limitante para $v_{1}$ não é $1 \mathrm{~m}$ número cabalístico, simplesmente é um valor que escolhemos para não tornar este capítulo repleto de cálculos minuciosos e cansativos. A Seção 6 contém nossas tabelas. Finalmente, na última seção, respondemos negativamente a uma questão proposta por Zariski em [Z2], relativa aos semigrupos do tipo $\left\langle v_{0}, v_{0}+1\right\rangle$, dando um contraexemplo para o semigrupo $\langle 7,8\rangle$. Aproveitamos a oportunidade para classificar os semigrupos $\langle 6,7\rangle$ e $\langle 7,8\rangle$, obtendo condições necessárias e suficientes, nestes casos, para a validade do enunciado proposto por Zariski. 


\subsection{Cuvas̈ com Multiplicidade $\leq 3$}

Note que se $v_{0}=1$, então $x=t$ e $\mathcal{O}=\overline{\mathcal{O}}=K[[t]]$. Portanto, todos os ramos de multiplicidade 1 são equivalentes.

Se $C$ é um ramo com $v_{0}=2$, então o semigrupo associado é da forma $\Gamma=\left\langle 2, v_{1}\right\rangle$, cujo condutor é $\mu=v_{1}-1$, ou seja, não pode haver invariante $\lambda$ de Zariski por causa da limitação $v_{1}<\lambda<\mu$ (Veja parágrafo após a Observação 3.2). Portanto, $\Lambda \backslash \Gamma=\{\}$ e $C$ é equivalente à

$$
\left\{\begin{array}{l}
x=t^{2} \\
y=t^{v_{1}}
\end{array}\right.
$$

Consideremos agora os ramos, com $v_{0}=3$. Assim, o semigrupo associado é $\Gamma=\left\langle 3, v_{1}\right\rangle$.

Pelạ Proposição 4.2, podemos representar tais ramos por

$$
C:\left\{\begin{array}{l}
x=t^{3} \\
y=t^{v_{1}}+\sum_{i=2}^{\left[\frac{v_{1}}{3}\right]} a_{i} t^{2 v_{1}-3}\left(\left[\frac{v_{1}}{3}\right]+2-i\right) .
\end{array}\right.
$$

Se $a_{i}=0$ para todo $i=2, \ldots,\left[\frac{v_{1}}{3}\right]$, então o ramo $C$ é equivalente a

$$
\left\{\begin{array}{l}
x=t^{3} \\
y=t^{v_{1}}
\end{array}\right.
$$

e $\Lambda \backslash \Gamma=\{\}$.

Caso contrário, considere o menor índice $k$ para o qual $a_{k} \neq 0$, i.e.,

$$
C:\left\{\begin{array}{l}
x=t^{3} \\
y=t^{v_{1}}+\sum_{i=k}^{\left[\frac{v_{1}}{3}\right]} a_{i} t^{2 v_{1}-3}\left(\left[\frac{v_{1}}{3}\right]+2-i\right)
\end{array}\right.
$$

Desse modo, $\lambda_{k}=2 v_{1}-3\left(\left[\frac{v_{1}}{3}\right]+2-k\right)$ é o invariante de Zariski para o ramo $C$. Usando a Proposição 4.3 e uma homotetia (cf. Exemplo 3.1), podemos considerar

$$
C_{k}:\left\{\begin{array}{l}
x=t^{3} \\
y=t^{v_{1}}+t^{\lambda_{k}}
\end{array}\right.
$$

É óbvio que duas curvas $C_{k}$ e $C_{j}$, como acima, são equivalentes se, e somente se, $k=j$, ou seja, possuem o mesmo invariante de Zariski. 
Veja que, independentemente do valor de $k$, o invariante $\lambda_{k}$ de Zariski satisfaz às hipóteses do Corolário 5.1. Portanto, se $\lambda=2 v_{1}-3 i \operatorname{com} 2 \leq i \leq$ $\left[\frac{v_{1}}{3}\right]$ e $\Omega_{1}=x d y-\frac{v_{1}}{3} y d x$, então

$$
\Lambda \backslash \Gamma=\left\{v\left(\Omega_{1}\right)+1+3 j ; 0 \leq j \leq i-2\right\}=\{\lambda+3 j ; 1 \leq j \leq i-1\} .
$$

\subsection{Lacunas Especiais para Multiplicidade 4}

$\mathrm{O}$ caso $v_{0}=4$ não é tão simples quanto os anteriores. De fato, a primeira diferença significativa é que podemos ter semigrupos com $v_{0}=4$ de gênero 1 ou gênero 2 .

Inicialmentc, vamos considerar os semigrupos de gênero 1 , on seja, aque]es da forma $\Gamma=\left\langle 4, v_{1}\right\rangle$.

Podemos considerar que $o$ invariante $\lambda$ de Zariski está presente na parametrização de tais curvas, pois caso contrário, a curva seria equivalente a

$$
\left\{\begin{array}{l}
x=t^{4} \\
y=t^{v_{1}}
\end{array}\right.
$$

e $\Lambda \backslash \Gamma=\{\}$

Na parametrização de um ramo com semigrupo $\Gamma=\left\langle 4, v_{1}\right\rangle$, podemos considerar apenas algumas lacunas de $\Gamma$ maiores que $v_{1}$, que de acordo com a Proposição 4.2 e a Observação 3.2 são

$$
\begin{aligned}
& 2 v_{1}-4 j \text { com } 2 \leq j \leq\left[\frac{v_{1}}{4}\right], \\
& 3 v_{1}-4 j \text { com } 2 \leq j \leq\left[\frac{v_{1}}{2}\right] .
\end{aligned}
$$

Caso a) $\lambda=3 v_{1}-4 j$, para algum $j=2, \ldots,\left[\frac{v_{1}}{2}\right]$.

Pelo Teorema 4.1 e por uma homotetia (Veja Exemplo 3.1), podemos, nesse caso, supor a curva dada por uma parametrização da forma

$$
\left\{\begin{array}{l}
x=t^{4} \\
y=t^{v_{1}}+t^{3 v_{1}-4 j}+\sum_{i=2}^{j-\left[\frac{v_{1}}{4}\right]-1} a_{i} t^{2 v_{1}-4\left(j-\left[\frac{v_{1}}{4}\right]+1-i\right)} .
\end{array}\right.
$$

Pelo Corolário 5.1, qualquer que seja o valor de $j$ teremos apenas uma DNEM, a saber $\Omega_{1}=x d y-\frac{v_{1}}{4} y d x$ e portanto,

$$
\Lambda \backslash \Gamma=\{\lambda+4 i ; 1 \leq i \leq j-1\} .
$$


Note que se $v_{1}<8$, então a parametrização acima se resume a

$$
\left\{\begin{array}{l}
x=t^{4} \\
y=t^{v_{1}}+t^{\lambda}
\end{array}\right.
$$

e temos apenas uma classe de equivalência.

A análise da equivalência para os ramos da forma (6.1) será feita posteriormente.

Caso b) $\lambda=2 v_{1}-4 j$, para algum $j=2, \ldots,\left[\frac{v_{1}}{4}\right]$.

O Teorema 4.1 e o Exemplo 3.1 garantem que, nessa situação, todos os ramos podem ser representados por uma parametrização da forma

$$
\left\{\begin{array}{l}
x=t^{4} \\
y=t^{v_{1}}+t^{2 v_{1}-1 j}+\sum_{i=1}^{\left[\frac{v_{1}}{4}\right]} a_{i} t^{3 n_{1}-1}\left(\left[\frac{v_{1}}{4}\right]+j+1-i\right) .
\end{array}\right.
$$

O Teorema 5.2 permite determinar todos os possíveis conjuntos de lacunas especiais. No entanto, aplicaremos o algoritmo do Teorema 2.2, juntamente com a Proposição 3.3, para encontrar quais restrições sobre os parâmetros $a_{i}, \operatorname{com} i=1, \ldots,\left[\frac{v_{1}}{4}\right]$, devemos impor para obter determinados conjuntos de lacunas especiais.

Temos portanto, $\Omega_{1}=x d y-\frac{v_{1}}{4} y d x$, e conseqüentemente,

$$
\begin{aligned}
\psi\left(\Omega_{1}\right)= & \left(v_{1}-4 j\right) t^{2 v_{1}-4(j-1)-1}+ \\
& +\sum_{i=1}^{\left[\frac{v_{1}}{4}\right]}\left(2 v_{1}-4\left(\left[\frac{v_{1}}{4}\right]+j+1-i\right)\right) a_{i} t^{3 v_{1}-4\left(\left[\frac{v_{1}}{4}\right]+j-i\right)-1},
\end{aligned}
$$

onde $\psi$ é o homomorfismo definido em $(2.2)$ e $v\left(\Omega_{1}\right)+1=2 v_{1}-4(j-1)$.

Uma vez que tomando $B=\left\{d x, d y, \Omega_{1}\right\}$, a lacuna limitante é $l=3 v_{1}-4 j$. O próximo $S$-processo a ser considerado é o de menor altura dentre,

$$
y^{2} \Omega_{1}-\frac{v_{1}-4 j}{4} x^{v_{1}-j} d x
$$

e

$$
v_{1} x^{j-1} \Omega_{1}-\left(v_{1}-4 j\right) y d y .
$$

Como a ordem da altura do primeiro $S$-processo acima supera o condutor $\mu$ de $\Gamma$, pois

$$
2 v_{1}+2 v_{1}-4(j-1)-1 \geq 4 v_{1}+4-4\left[\frac{v_{1}}{4}\right]-1>3 v_{1}>\mu=3\left(v_{1}-1\right)
$$


e como a ordem da altura do segundo $S$-processo é $4(j-1)+2 v_{1}-4(j-1)=$ $2 v_{1}$, temos que

$$
\Omega_{2}=v_{1} x^{j-1} \Omega_{1}-\left(v_{1}-4 j\right) y d y
$$

e conseqüentemente,

$$
\begin{aligned}
\psi\left(\Omega_{2}\right)= & \sum_{i=1}^{\left[\frac{v_{1}}{4}\right]-j} v_{1} a_{i}\left(2 v_{1}-4\left(\left[\frac{v_{1}}{4}\right]+j+1-i\right)\right) t^{3 v_{1}-4\left(\left[\frac{v_{1}}{4}\right]+1-i\right)-1}+ \\
& +\left(v_{1} a_{\left[\frac{v_{1}}{4}\right]+1-j}\left(2 v_{1}-8 j\right)-\left(v_{1}-4 j\right)\left(3 v_{1}-4 j\right)\right) t^{3 v_{1}+1 j-1}+\cdots
\end{aligned}
$$

Se existe $a_{i} \neq 0$ para algım $i=1, \ldots,\left[\frac{v_{1}}{4}\right]-j$, então $\Omega_{2}$ é uma DNEM, $\operatorname{com} v\left(\Omega_{2}\right)+1=3 v_{1}-4\left(\left[\frac{v_{1}}{4}\right]+1-k\right)$, onde $k=\min \left\{i ; a_{i} \neq 0\right\}$. Assim, o algoritmo finaliza, pois temos $v_{0}-2$ DNEM's, que pela Observação 3.3, sabemos ser o número máximo de DNEM's possíveis.

Nesse caso, temos

$$
\begin{aligned}
\Lambda \backslash \Gamma & =\left\{v\left(\Omega_{1}\right)+1+\gamma \notin \Gamma ; \gamma \in \Gamma\right\} \cup\left\{v\left(\Omega_{2}\right)+1+\gamma \notin \Gamma ; \gamma \in \Gamma\right\}= \\
& =\left\{2 v_{1}-4 i ; 1 \leq i \leq j-1\right\} \cup\left\{3 v_{1}-4 i ; 1 \leq i \leq\left[\frac{v_{1}}{4}\right]+1-k\right\} .
\end{aligned}
$$

Se $a_{i}=0$ para todo $i \operatorname{com} 1 \leq i \leq\left[\frac{v_{1}}{4}\right]-j$ e $a_{\left[\frac{v_{1}}{4}\right]+1-j} \neq \frac{v_{1}+\lambda}{2 v_{1}}$, então $\Omega_{2}$ é DNEM com $v\left(\Omega_{2}\right)+1=3 v_{1}-4 j$. Novamente, o algoritmo finaliza, pois obtemos o número máximo de DNEM's.

Nessa situação, temos

$$
\Lambda \backslash \Gamma=\left\{2 v_{1}-4 i ; 1 \leq i \leq j-1\right\} \cup\left\{3 v_{1}-4 i ; 1 \leq i \leq\left[\frac{v_{1}}{4}\right]+1-j\right\}
$$

Por outro lado, se $a_{i}=0$ para todo $i=1, \ldots,\left[\frac{v_{1}}{4}\right]-j$ e $a_{\left[\frac{v_{1}}{4}\right]+1-j}=\frac{v_{1}+\lambda}{2 v_{1}}$, então a ordem de $\Omega_{2}$ é maior ou igual à lacuna limitante, o que indica o fim do algoritmo. Nessas condições, teremos que $\Omega_{1}$ é a única DNEM e

$$
\Lambda \backslash \Gamma=\left\{2 v_{1}-4 i ; 1 \leq i \leq j-1\right\} \cup\left\{3 v_{1}-4 i ; 1 \leq i \leq j-1\right\}
$$

Se nosso interesse fosse apenas a análise da equidiferenciabilidade, então o estudo estaria concluido. 


\subsection{Formas Normais para Multiplicidade 4}

Vamos mostrar que se um ramo da forma (6.2) admite uma DNEM $\Omega_{2}=$ $v_{1} x^{j-1} \Omega_{1}-\left(v_{1}-4 j\right) y d y$, então podemos eliminar todos os termos com ordem $4(\alpha-1)+v\left(\Omega_{2}\right)+1$ com $\alpha \geq 0$ que figuram na parametrização.

Observe que para os ramos com invariante de Zariski da forma $\lambda=2 v_{1}-4 j$ com $2 \leq j \leq\left[\frac{v_{1}}{4}\right]$, todos os termos de ordem maior ou igual a $3 v_{1}-4 j$ são elimináveis de modo a deixar os termos de ordem inferior inalterados, pois se $\gamma \geq 3 v_{1}-4 j$, então $\gamma \in \Gamma \cup\left(\Gamma+v_{1}-4\right) \cup\left(\Gamma^{*}+v\left(\Omega_{1}\right)+1-4\right)$ e a afirmação segue do Teorema 4.1 .

Escrevamos $\Omega_{2}$ na forma $q d x-p d y$, ou seja,

$$
\Omega_{2}=v_{1} x^{j-1} \Omega_{1}-\left(v_{1}-4 j\right) y d y=-\frac{v_{1}^{2}}{4} x^{j-1} y d x-\left(-v_{1} x^{j}+\left(v_{1}-4 j\right) y\right) d y
$$

e cousideremos para $\beta$ arbitrário, a mudança de coordenadas

$$
\begin{aligned}
& t_{1}=t\left(1+\frac{\beta x^{\alpha} p}{t^{4}}\right)^{\frac{1}{4}} \\
& y_{1}=y+\beta x^{\alpha} q .
\end{aligned}
$$

Como vimos 110 Exemplo 3.2, tal mudauça nos dá

$$
\begin{aligned}
& x_{1}=t_{1}^{4} \\
& y_{1}=t_{1}^{v_{1}}+t_{1}^{\lambda}+\sum_{i=k}^{\left[\frac{v_{1}}{4}\right]} a_{i} t_{1}^{3 v_{1}-4\left(\left[\frac{v_{1}}{4}\right]+j+1-i\right)}+\frac{\beta x^{\alpha} \Omega_{2}}{d x}- \\
& -t^{v_{1}}\left(\sum_{s=2}^{\infty}\left(\begin{array}{c}
\frac{v_{1}}{4} \\
s
\end{array}\right)\left(\frac{\beta x^{\alpha} p}{t^{4}}\right)^{s}\right)-t^{\lambda}\left(\sum_{s=2}^{\infty}\left(\begin{array}{l}
\frac{\lambda}{4} \\
s
\end{array}\right)^{d x}\left(\frac{\beta x^{\alpha} p}{t^{4}}\right)^{s}\right)-
\end{aligned}
$$

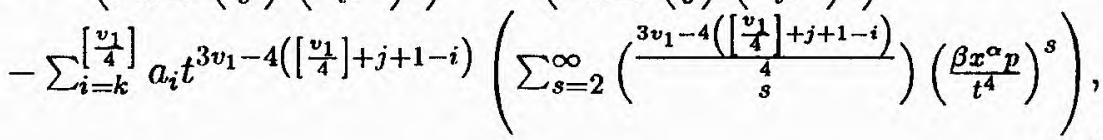

onde $k=\min \left\{i ; a_{i} \neq 0\right\}$.

Vejamos mais de perto cada parcela da última expressão acima que é da forma

$$
A_{r}=a_{i} t^{r} \sum_{s=2}^{\infty}\left(\begin{array}{l}
\frac{r}{4} \\
s
\end{array}\right)\left(\frac{\beta x^{\alpha} p}{t^{4}}\right)^{s}
$$

Se $r \in\left\{3 v_{1}-4\left(\left[\frac{v_{1}}{4}\right]+j+1-i\right) ; i=k, \ldots,\left[\frac{v_{1}}{4}\right]\right\}$, então como $s \geq 2$ e $\alpha \geq 0$ temos

$$
\begin{aligned}
v\left(A_{r}\right) & =3 v_{1}-4\left(\left[\frac{v_{1}}{4}\right]+j+1-i\right)+8 j+8(\alpha-1)= \\
& =3 v_{1}-4\left(\left[\frac{v_{1}}{4}\right]+1-i\right)+4 j+8(\alpha-1)=
\end{aligned}
$$




$$
=\left\{\begin{array}{c}
3 v_{1}-4\left(\left[\frac{v_{1}}{4}\right]+1-k\right)+4 j+8(\alpha-1)> \\
>3 v_{1}-4\left(\left\lceil\frac{v_{1}}{4}\right]+1-k\right)+4(\alpha-1)= \\
=4(\alpha-1)+v\left(\Omega_{2}\right)+1 \\
\text { se } k=\min \left\{i ; a_{i} \neq 0\right\} \\
\text { com } i \leq k \leq\left[\frac{v_{1}}{4}\right]-j \\
3 v_{1}-4\left(\left[\frac{v_{1}}{4}\right]+1-\left[\frac{v_{1}}{4}\right]+j-1\right)+4 j+8(\alpha-1)= \\
=3 v_{1}-4 j+4(\alpha-1)+4 j+4(\alpha-1)> \\
>3 v_{1}-4 j+4(\alpha-1)=4(\alpha-1)+v\left(\Omega_{2}\right)+1 \\
\text { se } a_{i}=0 \text { para } \\
\text { todo } i \leq k \leq\left[\frac{v_{1}}{4}\right]-j
\end{array}\right.
$$

ou seja, não precisamos nos preocupar com cssas parcelas, pois a ordem destas superam a ordem do termo que desejamos eliminar.

Veja que, para $s \geq 2$, temos

$$
\begin{aligned}
t^{r}\left(\frac{x^{\alpha} p}{t^{4}}\right)^{s}= & t^{r} x^{\alpha s}\left(\left(-v_{1}\right)^{s} x^{s(j-1)}+\right. \\
& \left.+s\left(-v_{1}\right)^{s-1}\left(v_{1}-4 j\right) x^{(s-1)(j-1)-1} y+\begin{array}{c}
\text { termos de ordem } \\
\geq 2 v_{1}-4 j
\end{array}\right) \\
= & t^{4 \alpha s+r}\left(\left(-v_{1}\right)^{s} t^{4 s(j-1)}+s\left(-v_{1}\right)^{s-1}\left(v_{1}-4 j\right)\left(t^{v_{1}+4((s-1)(j-1)-1)}+\right.\right. \\
& \left.\left.+t^{\lambda+4((s-1)(j-1)-1)}\right)+\begin{array}{c}
\text { termos de ordem } \\
>2 v_{1}-4
\end{array}\right) .
\end{aligned}
$$

Se $r=v_{1}$, temos

$$
\begin{aligned}
t^{v_{1}}\left(\frac{x^{\alpha} p}{t^{4}}\right)^{s} & =\left(-v_{1}\right)^{s} t^{4 s(j-1+\alpha)+v_{1}}+ \\
& +s\left(-v_{1}\right)^{s-1}\left(v_{1}-4 j\right) t^{2 v_{1}+4(\alpha s+s(j-1)-j)}+\begin{array}{c}
\text { termos de ordem } \\
>3 v_{1}-4 i
\end{array}
\end{aligned}
$$

Se $r=\lambda=2 v_{1}-4 j$, temos

$$
t^{\lambda}\left(\frac{x^{\alpha} p}{t^{4}}\right)^{s}=\left(-v_{1}\right)^{s} t^{2 v_{1}+4(\alpha s+s(j-1)-j)}+\begin{gathered}
\text { termos com ordem } \\
\geq 3 v_{1}-4 j
\end{gathered}
$$


Note que $s(j-1)-j \geq 0$, pois $s \geq 2$ e $j \geq 2$, ou seja,

$$
2 v_{1}+4(\alpha s+s(j-1)-j) \in\left\langle 4, v_{1}\right\rangle \text {. }
$$

-Podemos reescrever (6.4) como

$$
\begin{aligned}
x_{1}= & t_{1}^{4} \\
y_{1}= & t_{1}^{v_{1}}+t_{1}^{\lambda}+\sum_{i=k}^{\left[\frac{v_{1}}{4}\right]} a_{i} t_{1}^{3 v_{1}-4\left(\left[\frac{v_{1}}{4}\right]+j+1-i\right)}+\frac{\beta x^{\alpha} \Omega_{2}}{d x}- \\
& -\sum_{s=2}^{\infty}\left(\left(\begin{array}{c}
\frac{v_{1}}{4} \\
s
\end{array}\right)\left(-v_{1}\right)^{s} t^{v_{1}+4 s(\alpha+j-1)}+\right. \\
& \left.\quad+\left(-v_{1}\right)^{s-1}\left(\left(\begin{array}{c}
\frac{v_{1}}{4} \\
s
\end{array}\right) s\left(v_{1}-4 j\right)-\left(\begin{array}{c}
\frac{\lambda}{4} \\
s
\end{array}\right) v_{1}\right) t^{2 v_{1}+4(\alpha s+s(j-1)-j)}\right)+
\end{aligned}
$$

+ termos de ordem superior a $v\left(\frac{x^{\alpha} \Omega_{2}}{d x}\right)$.

Reservemos a expressão acima e analisemos as expansões de $x^{s(\alpha+j-1)} y$ e $x^{\alpha s+s(j-1)-j} y^{2}$ para $\alpha \geq 0$ e $s \geq 2$. Por uma análise semelhante àquela feita em (6.5), temos

$$
\begin{aligned}
& x^{s(\alpha+j-1)} y=t^{v_{1}+4 s(\alpha+j-1)}+t^{2 v_{1}-4 j+4 s(\alpha+j-1)}+\left[\begin{array}{c}
\text { termos de ordem } \\
>v\left(\frac{x^{\alpha} \Omega_{2}}{d x}\right)
\end{array}\right. \\
& x^{\alpha s+s(j-1)-j} \eta^{2}=t^{2 v_{1}+4(\alpha s+s(j-1)-j)}+\begin{array}{c}
\text { termos de ordem } \\
>3 v_{1}-4 j
\end{array}
\end{aligned}
$$

Assim, se ao invés de considerarmos a mudança de coordenadas dada em (6.3), tomarmos

$$
\begin{aligned}
& t_{1}=t\left(1+\frac{\beta x^{\alpha} p}{t^{4}}\right)^{\frac{2}{4}} \\
& y_{1}=y+\beta x^{\alpha} q+\sum_{s=2}^{\infty}\left(\gamma_{s} x^{s(j-1)}+\delta_{s} x^{s(j-1)-j} y\right) x^{\alpha s} y
\end{aligned}
$$

podemos escolher $\beta, \gamma_{s}$ e $\delta_{s}$ de modo a eliminar o termo de ordem $v\left(\frac{x^{\alpha} \Omega_{2}}{d x}\right)$ com $\alpha \geq 0$, sem que alteremos os termos de ordem inferior.

Portanto, se em (6.2) existe $a_{i} \neq 0$ para algum $i=1, \ldots,\left[\frac{v_{1}}{4}\right]-j$, então podemos nos redizir a ramos da forma

$$
\left\{\begin{array}{l}
\cdot x=t^{4} \cdot \\
y=t^{v_{1}}+t^{2 v_{1}-4 j}+\sum_{i=k}^{j-2+k} a_{i} t^{3 v_{1}-4}\left(\left[\frac{v_{1}}{4}\right]+j+1-i\right),
\end{array}\right.
$$


$\operatorname{com} k=\min \left\{i ; a_{i} \neq 0\right\}$ e cujo conjunto de lacunas especiais, conforme vimos ua Seção 2, é

$\Lambda \backslash \Gamma=\left\{2 v_{1}-4 i ; 1 \leq i \leq j-1\right\} \cup\left\{3 v_{1}-.4 i ; 1 \leq i \leq\left[\frac{v_{1}}{4}\right]+1-k\right\}$.

Se $a_{i}=0$ para todo $i=1, \ldots,\left[\frac{v_{1}}{4}\right]-j$ e $a_{\left[\frac{v_{1}}{4}\right]-j+1} \neq \frac{v_{1}+\lambda}{2 v_{1}}$, então podemos nos reduzir a

$$
\left\{\begin{array}{l}
x=t^{4} \\
y=t^{v_{1}}+t^{2 v_{1}-1 j}+\sum_{i=\left[\frac{v_{1}}{4}\right]-j+1}^{\left[\frac{v_{1}}{4}\right]-1} a_{i} t^{3 v_{1}-4}\left(\left[\frac{v_{1}}{4}\right]+j+1-i\right)
\end{array}\right.
$$

e, conforme vimos na Seção 2 .

$$
\Lambda \backslash \Gamma=\left\{2 v_{1}-4 i ; 1 \leq i \leq j-1\right\} \cup\left\{3 v_{1}-4 i ; 1 \leq i \leq\left[\frac{v_{1}}{4}\right]+1-j\right\} .
$$

Por outro lado, se $a_{i}=0$ para todo $i=1, \ldots,\left[\frac{v_{1}}{4}\right]-j$ e $a_{\left[\frac{v_{1}}{4}\right]-j+1}=\frac{v_{1}+\lambda}{2 v_{1}}$, então basta considerar os ramos da forma

$$
\left\{\begin{array}{l}
x=t^{4} \\
y=t^{v_{1}}+t^{2 v_{1}-4 j}+\left(\frac{\lambda+v_{1}}{2 v_{1}}\right) t^{3 v_{1}-8 j}+\sum_{i=\left[\frac{v_{1}}{4}\right]-j+2}^{\left[\frac{v_{1}}{4}\right]} a_{i} t^{3 v_{1}-4\left(\left[\frac{v_{1}}{4}\right]+j+1-i\right)}
\end{array}\right.
$$

onde

$$
\Lambda \backslash \Gamma=\left\{2 v_{1}-4 i ; 1 \leq i \leq j-1\right\} \cup\left\{3 v_{1}-4 i ; 1 \leq i \leq j-1\right\} .
$$

No caso de ramos com $v_{0}=4$ e gênero 2, o Exemplo 4.1 permite que nos restrinjamos aos ramos da forma

$$
\left\{\begin{array}{l}
x=t^{4} \\
y=t^{v_{1}}+t^{v_{2}+v_{1}-4 m_{1}}+\sum_{k=2}^{\left[\frac{v_{1}}{4}\right]} a_{k} t^{v_{2}-4}\left(\left[\frac{v_{1}}{4}\right]+2-k\right),
\end{array}\right.
$$

com $m_{1}=\frac{v_{1}}{2}$. Neste caso, como $\Omega_{1}=x d y-\frac{v_{1}}{v_{0}} y d x$ é a única DNEM, o conjunto das lacunas especiais é

$$
\Lambda \backslash \Gamma=\left\{v_{2}+v_{1}-4 i ; 1 \leq i \leq m_{1}-1\right\} .
$$

No que segue, nos referiremos às últimas quatro parametrizações acima como formas normais para os ramos de multiplicidade 4 . 
Observe que em todas as formas normais acima, os expoentes superiores a $\lambda$ que podem estar presentes na parametrização não pertencem a $v\left(\frac{\mathcal{O} d \mathcal{O}}{d x}\right)$. Em particular, os termos de ordem maiores que $l-4$, onde $l$ é a lacuna limitante de $\Lambda=v(\mathcal{O} d \mathcal{O})+1$, podem ser simplesmente desprezados, ou seja, se

$$
C_{1}:\left\{\begin{array}{l}
x_{1}=t^{4} \\
y_{1}=t^{v_{1}}+\sum_{i>v_{1}} a_{i} t^{i}
\end{array} \quad C_{2}:\left\{\begin{array}{l}
x_{2}=t^{4} \\
y_{2}=t^{v_{1}}+\sum_{i>v_{1}} a_{i}^{\prime} t^{i}
\end{array}\right.\right.
$$

e. $y_{1}=y_{2} \bmod t^{l-1+1}$, então $C_{1} \sim C_{2}$. De fato, se $\delta>l-4$, então existe. $\Omega \in \mathcal{O} d \mathcal{O}$ tal que $v(\Omega)+1=\delta+4>l$. Assim, $v\left(\frac{\Omega}{d x}\right)=\delta$ e, como vimos, todos os termos cuja ordem pertence a $v\left(\frac{\mathcal{O} d \mathcal{O}}{d x}\right)$ podem ser eliminados sem que alteremos os termos de ordem inferior.

\subsection{Classificação das Curvas de Multiplici- dade 4}

Obviamente, dois ramos que possuem $\Lambda \backslash \Gamma$ distintos não podem ser equivalentes (Proposição 3.1). Mostraremos que, dois ramos que possuem o mesmo conjunto de lacunas especiais e estão na forma normal descrita acima, são equivalentes se, e somente se, diferem por uma homotetia, i.e., as únicas mudanças de coordenadas que preservam os geradores dos Anéis de Puiseux na forma normal são aquelas apresentadas no Exemplo 3.1, que são em número finito. De fato, como as formas normais dadas anteriormente possuem coeficiente de $t^{\lambda}$ igual a 1 e nas homotetias

$$
\begin{aligned}
& t_{1}=c t \\
& y_{1}=c^{v_{1}} y
\end{aligned}
$$

devemos ter $c \in K^{*} \operatorname{com} c^{v_{1}-\lambda}=1$, segue que temos apenas $\lambda-v_{1}$ homotetias que preservam as formas normais apresentadas.

A estratégia que usaremos para mostrar que as únicas mudanças de coordenadas que preservam as formas normais são as homotetias, é mostrar que mudanças de coordenadas da forma

$$
\begin{aligned}
& t_{1}=t\left(1+\frac{p}{t^{4}}\right)^{\frac{1}{4}} \\
& y_{1}=y+q,
\end{aligned}
$$

$\operatorname{com} p, q \in \mathcal{O}, v(p)>4$ e $v(q)>v_{1}$, que preservam formas normais agem como a identidade, ou seja, não alteram os coeficientes nem eliminam e/ou 
introduzem termos com ordens inferiores a $l-4$, onde $l$ é a lacuna limitante. de $v(\mathcal{O} d \mathcal{O})+1$. Dessa forma, como toda mundança de coordenadas é uma composição de uma homotetia (Veja Exemplo 3.1) e uma mudança de coordenadas como no Exemplo 3.2, segue que apenas as homotetias nos dão ramos equivalentes com mesmo tipo de forma normal.

Inicialmente, note que ao efetuarmos ıma mudança da forma

$$
\begin{aligned}
& t_{1}=t\left(1+\frac{p}{t^{4}}\right)^{\frac{1}{4}} \\
& y_{1}=y+q
\end{aligned}
$$

teremos

$$
\left\{\begin{array}{l}
x_{1}=t_{1}^{4} \\
y_{1}=y\left(t_{1}\right)+A(t)
\end{array}\right.
$$

com

$$
A(t)=q-\frac{p d y}{d x}-\sum_{i \geq v_{1}} a_{i} t^{i} \sum_{k=2}^{\infty}\left(\begin{array}{l}
\frac{i}{4} \\
k
\end{array}\right)\left(\frac{p}{t^{4}}\right)^{k}
$$

onde $y=\sum_{i \geq v_{1}} a_{i} t^{i}$ está em uma das formas normais apresentadas anteriormente.

Basta nos preocuparmos com os termos de $A(t)$ com ordem menor on igual a $l-4$, onde $l$ é a lacuna limitante de $v(\mathcal{O} d \mathcal{O})+1$ pois, como vimos, os termos de ordem superior a $l-4$ podem ser eliminados sem que alteremos os de ordem inferior.

Vimos na seção anterior, através da aplicação do algoritmo para obter uma Base Standard Mínima para $\mathcal{O} d \mathcal{O}$ que

$$
\begin{aligned}
& \lambda=3 v_{1}-4 j \quad \Longrightarrow l= \begin{cases}\lambda & \text { se } v_{1}<8 \\
2 v_{1}-4 & \text { se } v_{1}>8,\end{cases} \\
& \lambda=2 v_{1}-4 j \quad \Longrightarrow l \leq 3 v_{1}-4 j, \\
& \lambda=v_{2}+v_{1}-4 m_{1} \Longrightarrow l= \begin{cases}\lambda & \text { se } v_{1}<8 \\
v_{2}-4 & \text { se } v_{1}>8\end{cases}
\end{aligned}
$$

Observe que se $\lambda \neq 2 v_{1}-4 j$ e $v_{1}<8$, então as formas normais apresentadas auteriormente se resumem a

$$
\left\{\begin{array}{l}
x=t^{4} \\
y=t^{v_{1}}+t^{\lambda}
\end{array}\right.
$$


e a análise da equivalência é imediata. Assim, nas análises que realizaremos a seguir estaremos considerando $v_{1}>8 \mathrm{e}$

$$
l \begin{cases}=2 v_{1}-4 & \text { se } \lambda=3 v_{1}-4 j \\ \leq 3 v_{1}-4 j & \text { se } \lambda=2 v_{1}-4 j \\ =v_{2}-4 & \text { se } \lambda=v_{2}+v_{1}-4 m_{1}\end{cases}
$$

Para estudarmos os termos de ordens menores on ignais a $l-4 \mathrm{em} A(t)$, basta considerarmos

$$
\begin{aligned}
& p= \begin{cases}\sum_{\alpha=2}^{\left[\frac{v_{1}}{4}\right]} b_{\alpha-1} x^{\alpha} & \text { se } \lambda=3 v_{1}-4 j \\
\sum_{\alpha=2}^{\left[\frac{v_{1}}{4}\right]-1} b_{\alpha-1} x^{\alpha}+\sum_{\beta=0}^{\left[\frac{v_{1}}{4}\right]}\left(c_{\beta} x^{\left[\frac{v_{1}}{4}\right]+\beta}+d_{\beta} x^{\beta} y\right) & \text { se } \lambda=2 v_{1}-4 j \\
\sum_{\alpha}^{\left[\frac{v_{1}}{4}\right]-1} b_{\alpha-1} x^{\alpha}+\sum_{\beta=0}^{\left[\frac{v_{2}-v_{1}}{4}\right]}\left(c_{\beta} x^{\left[\frac{v_{1}}{4}\right]+\beta}+d_{\beta} x^{\beta} y\right) & \text { se } \lambda=v_{2}+v_{1}-4 m_{1}\end{cases}
\end{aligned}
$$

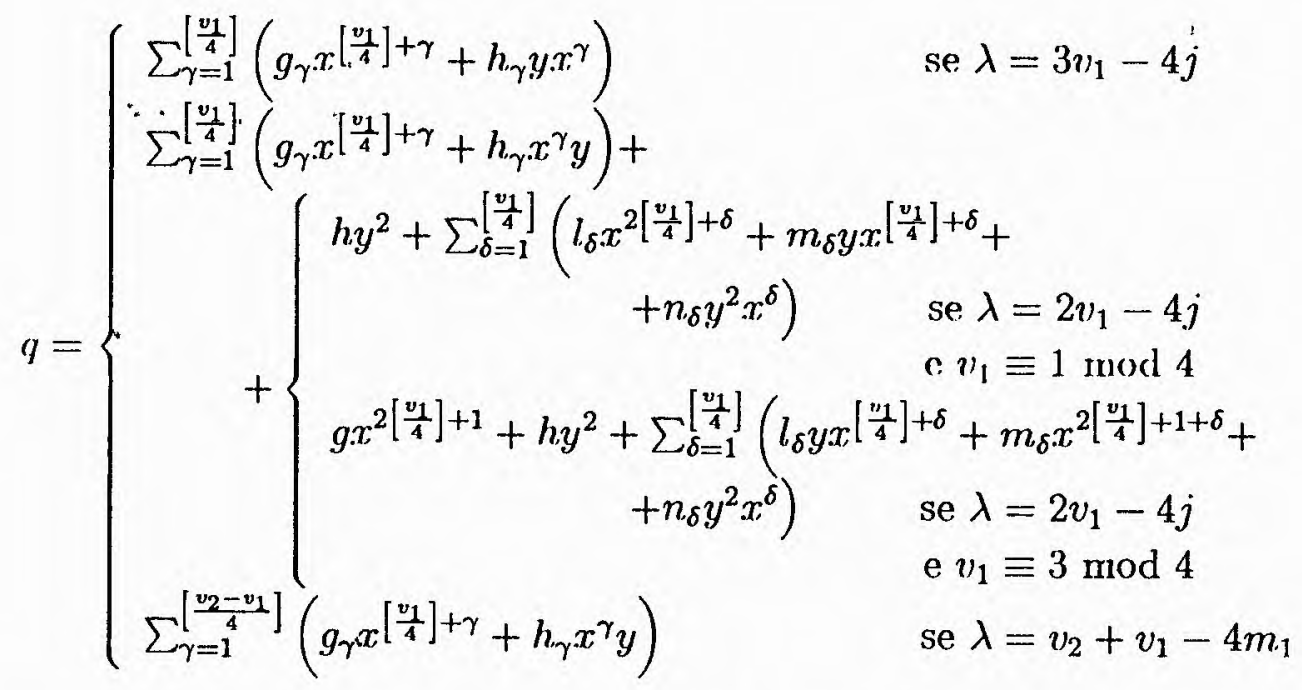

Note que em $A(t)$ o quociente $\frac{p}{t^{4}}$ é um fator relevante para nossa allálise. Vamos mostrar, para todo $k \geq 2$, que $\left(\frac{p}{t^{4}}\right)^{k}=B_{k} \bmod t^{l-v_{1}-v_{0}+1}$, para algum $B_{k} \in \mathcal{O}$.

Desse modo, usando que $\Omega_{1}=\sum_{i \geq \lambda}\left(\lambda-v_{1}\right) a_{i} t^{i+v_{0}-1}$ e a existência de $B_{k}$ como acima, podemos escrever

$$
A(t)=\frac{\omega}{d x}-\Upsilon \bmod t^{l-4+1}
$$


onde

$$
\omega=q d x-p d y-\left(\sum_{k=2}^{\infty}\left(\begin{array}{l}
\frac{v_{1}}{4} \\
k
\end{array}\right) B_{k}\right) y d x-\left(\sum_{k=2}^{\infty}\left(\left(\begin{array}{c}
\frac{\lambda}{4} \\
k
\end{array}\right)-\left(\begin{array}{c}
\frac{v_{1}}{4} \\
k
\end{array}\right)\right) B_{k}\right) \frac{v_{0}}{\lambda-v_{1}} \Omega_{1}
$$

e

$$
\Upsilon=\sum_{i>\lambda} a_{i} \sum_{k=2}^{\infty}\left(\left(\begin{array}{l}
\frac{i}{4} \\
k
\end{array}\right)-\left(\begin{array}{c}
\frac{v_{1}}{4} \\
k
\end{array}\right)-\frac{i-v_{1}}{\lambda-v_{1}}\left(\left(\begin{array}{c}
\frac{\lambda}{4} \\
k
\end{array}\right)-\left(\begin{array}{c}
\frac{v_{1}}{4} \\
k
\end{array}\right)\right)\right) B_{k} t^{i},
$$

onde obviamente $\omega \in \mathcal{O} d \mathcal{O}$.

Como queremos preservar a forma normal, que como observamos na Seçño 2 não possui termos de ordem em $v\left(\frac{\mathcal{O d O}}{d x}\right)$, vejamos que condições sobrr us parâmetros de $p$ e $q$ devemos impor para que $A(t)$ não introḍua tîrmos cujas ordens estiejam em $v\left(\frac{\mathcal{O d O}}{d x}\right)$.

Note que se $v\left(\frac{\omega}{d x}\right)<v(\Upsilon)$, então $v(A(t))=v\left(\frac{\omega}{d x}\right) \in v\left(\frac{\mathcal{O} d \mathcal{O}}{d x}\right)$, possibilidade que queremos descartar.

Suponhamos que $v\left(\frac{\omega}{d x}\right)=v(\Upsilon)=v\left(B_{2}\right)+\zeta$.

Como $\zeta$ é a menor ordem dos termos em $y$, acima'de $\lambda$, e como $y$ está numa das formas normais, temos que $\zeta \notin v\left(\frac{\mathcal{O d O}}{d x}\right)$. Por outro lado, por uma análise de cada forma normal, conclui-se que se $v\left(B_{2}\right)+\zeta=v\left(\frac{\omega}{d x}\right)\left(\in v\left(\frac{\mathcal{O} d \mathcal{O}}{d x}\right)\right)$, então $v\left(B_{2}\right)+\zeta>l-4$, já que $v\left(B_{2}\right) \in \Gamma$. Outra possibilidade a ser' descartada.

Portanto, se queremos preservar a forma normal de $y$, devemos ter

$$
v\left(\frac{\omega}{d x}\right)>v(\Upsilon)=v\left(B_{2}\right)+\zeta
$$

Vamos no que segue, analisando caso a caso segundo os valores de $\lambda$, mostrar que (6.8) implica $A(t)=0$. Logo a mudança de coordenadas (6.6) age como a identidade, restando apenas a ação das homotetias sobre cada forma normal.

$$
\text { Caso: } \lambda=3 v_{1}-4 j
$$

Inicialmente, veja que $\left(\frac{p}{t^{4}}\right)^{k} \in \mathcal{O}$ para todo $k$. Assim, pondo $B_{k}=\left(\frac{p}{i^{4}}\right)^{k}$, podemos reescrever (6.7) como

$$
\omega=\sum_{\gamma=1}^{\left[\frac{v_{1}}{4}\right]}\left(g_{\gamma} x^{\left[\frac{v_{1}}{4}\right]+\gamma}+h_{\gamma} x^{\gamma} y\right) d x-\sum_{\alpha=2}^{\left[\frac{v_{1}}{4}\right]} b_{\alpha-1} x^{\alpha} d y-
$$




$$
-\Theta_{1} y d x-\frac{v_{0}}{\lambda-v_{1}} \Theta_{2} \Omega_{1}
$$

onde

$$
\Theta_{1}=\sum_{k=2}^{\infty}\left(\begin{array}{c}
\frac{v_{1}}{4} \\
k
\end{array}\right)\left(\sum_{\alpha=2}^{\left[\frac{v_{1}}{4}\right]} b_{\alpha-1} x^{\alpha-1}\right)^{k}
$$

e

$$
\Theta_{2}=\sum_{k=2}^{\infty}\left(\left(\begin{array}{l}
\frac{\lambda}{4} \\
k
\end{array}\right)-\left(\begin{array}{c}
\frac{v_{1}}{4} \\
k
\end{array}\right)\right)\left(\sum_{\alpha=2}^{\left[\frac{v_{1}}{4}\right]} b_{\alpha-1} x^{\alpha-1}\right)^{k}
$$

Note que $v\left(\Theta_{1}\right)=v\left(\Theta_{2}\right)=8(\alpha-1)$, para algum $\alpha \geq 2$.

Veja que a desigualdade de (6.8), levando em conta (6.1), nos indica que

$$
\begin{aligned}
v(\omega)-v_{0}+1 & >v\left(B_{2}\right)+2 v_{1}-4\left(j-\left[\frac{v_{1}}{4}\right]-1\right)= \\
& >2 v_{1}-4 j+v_{1}-4+4+v\left(B_{2}\right)= \\
& =v\left(\frac{\Omega_{1}}{d x}\right)+v\left(B_{2}\right) .
\end{aligned}
$$

Portanto,

$$
v(\omega)>v\left(\Omega_{1}\right)+v\left(B_{2}\right)=\lambda+4+v\left(B_{2}\right)=\lambda+4+2(v(p)-4) \geq \lambda+12 .
$$

Assim, para que tenhamos a condição (6.8), devemos ter $g_{1}=0$ e $h_{1}=$ $\frac{v_{1}}{v_{0}} b_{1}$. Ou seja,

$$
\begin{aligned}
\omega= & h_{1} x \Omega_{1}+\sum_{\gamma=2}^{\left[\frac{v_{1}}{4}\right]}\left(g_{\gamma} x^{\left[\frac{\nu_{1}}{4}\right]+\gamma}+h_{\gamma} x^{\gamma} y\right) d x-\sum_{\alpha=3}^{\left[\frac{v_{1}}{4}\right]} b_{\alpha-1} x^{\alpha} d y- \\
& -\Theta_{1} y d x-\frac{v_{0}}{\lambda-v_{1}} \Theta_{2} \Omega_{1} .
\end{aligned}
$$

Uma vez que $\max \left\{v\left(x \Omega_{1}\right), v\left(x^{\left[\frac{v_{1}}{4}\right]+2} d x\right)\right\}<\lambda+4+v\left(B_{2}\right)$, devemos impor que $g_{2}=h_{1}=0$; mas isto implica que $b_{1}=0$.

Note que a condição $b_{1}=0$ eleva a ordem de $p$ e conseqüientemente eleva as ordens de $B_{2}, \Theta_{1}$ e $\Theta_{2}$. Assim, a condição (6.8) se reescreve como

$$
v(\omega)>\lambda+4+v\left(B_{2}\right) \geq \lambda+20 .
$$

Para que a condição acima seja satisfeita devemos impor $g_{2}=0$ e $h_{2}=$ $\frac{v_{1}}{v_{0}} b_{2}$. Temos portanto, 


$$
\begin{aligned}
\omega= & h_{2} x^{2} \Omega_{1}+\sum_{\gamma=3}^{\left[\frac{v_{1}}{4}\right]}\left(g_{\gamma} x^{\left[\frac{v_{1}}{4}\right]+\gamma}+h_{\gamma} x^{\gamma} y\right) d x-\sum_{\alpha=4}^{\left[\frac{v_{1}}{4}\right]} b_{\alpha-1} x^{\alpha} d y- \\
& -\Theta_{1} y d x-\frac{v_{0}}{\lambda-v_{1}} \Theta_{2} \Omega_{1} .
\end{aligned}
$$

Como $\max \left\{v\left(x^{2} \Omega_{1}\right), v\left(x^{\left[\frac{v_{1}}{v_{0}}\right]+3} d x\right)\right\}<\lambda+20$, devemos ter $g_{3}=h_{2}=0$. Mas a última igualdade implica que $b_{2}=0$, o que eleva a ordem de $p$ e consequientemente eleva as ordens de $B_{2}, \Theta_{1}$ e $\Theta_{2}$.

Continuando com o mesmo raciocínio, chega-se à conclusão que em algum momento $v(\Upsilon)=v\left(B_{2}\right)+\zeta>l-4$. Logo $\Upsilon \in \frac{\mathcal{O d O}}{d x}$ e conseqüentemente $A(t) \in \frac{\mathcal{O d O}}{d x}$, o que não é permitido, a menos que $A(t)=0$.

$$
\text { Caso: } \lambda=2 v_{1}-4 j
$$

Veja que, neste caso, para todo $k \geq 2$ temos

$$
\begin{aligned}
\left(\frac{p}{t^{4}}\right)^{k} & =\left(\sum_{\alpha=2}^{\left[\frac{v_{1}}{4}\right]} b_{\alpha-1} x^{\alpha-1}+\sum_{\beta=0}^{\left[\begin{array}{c}
v_{1} \\
4
\end{array}\right]}\left(d_{\beta} x^{\beta-1} y+c_{\beta} x^{\left[\frac{v_{1}}{4}\right]+\beta}\right)\right)^{k}= \\
& =(C+D)^{k}=\sum_{i=0}^{k}\left(\begin{array}{c}
k \\
i
\end{array}\right) C^{k-i} D^{i} .
\end{aligned}
$$

Como estamos considerando $v_{1}>.8$ e $k \geq 2$, para $i \geq 2$ temos $v\left(C^{k-i} D^{i}\right) \geq 2 v_{1}-8 \geq l-v_{1}$. Deste modo temos que $C^{k}+k C^{k-1} D \in \mathcal{O}$. Assim, $\left(\frac{p}{t^{4}}\right)^{k} \equiv B_{k}=(C+D)^{k} \bmod t^{l-v_{1}-v_{0}+1}$.

Substituindo as expressões de $p, q$ e $B_{k}$ em (6.7), temos.

$$
\begin{aligned}
& \omega=\left(\sum_{\gamma=1}^{\left[\frac{v_{1}}{4}\right]}\left(g_{\gamma} x^{\left[\frac{v_{1}}{4}\right]+\gamma}+h_{\gamma} y x^{\gamma}\right)+\right.
\end{aligned}
$$

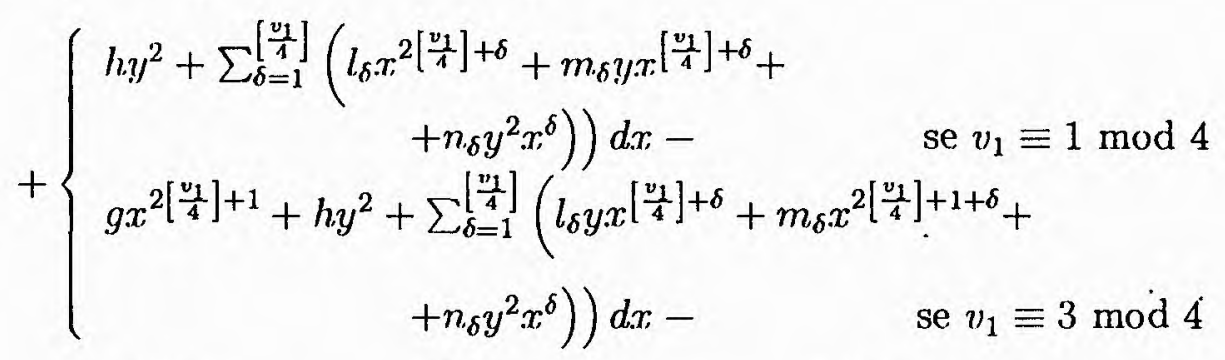

$$
\begin{aligned}
& -\left(\sum_{\alpha=2}^{\left[\frac{v_{1}}{4}\right]-1} b_{\alpha-1} x^{\alpha}+\sum_{\beta=0}^{\left[\frac{v_{1}}{4}\right]}\left(c_{\beta} x^{\left[\frac{v_{1}}{4}\right]+\beta}+d_{\beta} x^{\beta} y\right)\right) d y- \\
& -\Theta_{1} y d x-\frac{v_{0}}{\lambda-v_{1}} \Theta_{2} \Omega_{1} \bmod t^{l-4}
\end{aligned}
$$


onde

$$
\Theta_{1}=\sum_{k=2}^{\infty}\left(\begin{array}{l}
\frac{v_{1}}{4} \\
k
\end{array}\right)\left(\sum_{\alpha=2}^{\left[\frac{v_{1}}{4}\right]} b_{\alpha-1} x^{\alpha-1}+\sum_{\beta=0}^{\left[\frac{v_{1}}{4}\right]}\left(d_{\beta} \dot{x}^{\beta-1} y+c_{\beta} x^{\left[\frac{v_{1}}{4}\right]+\beta}\right)\right)^{k}
$$

e

$$
\Theta_{2}=\sum_{k=2}^{\infty}\left(\left(\begin{array}{c}
\frac{\lambda}{4} \\
k
\end{array}\right)-\left(\begin{array}{c}
v_{1} \\
k \\
k
\end{array}\right)\right)\left(\sum_{\alpha=2}^{\left[\frac{v_{1}}{4}\right]} b_{\alpha-1} x^{\alpha-1}+\sum_{\beta=0}^{\left[\frac{v_{1}}{4}\right]}\left(d_{\beta} x^{\beta-1} y+c_{\beta} x^{\left[\frac{v_{1}}{4}\right]+\beta}\right)\right)^{k}
$$

Note que

$$
\begin{aligned}
& v\left(x^{\left[\frac{v_{1}}{4}\right]+\gamma} d x\right)+1=4\left[\frac{v_{1}}{4}\right]+4 \gamma+4, \\
& v\left(y x^{\gamma} d x\right)+1=v_{1}+4 \gamma+4, \\
& v\left(\Theta_{1} y d x\right)+1=v_{1}+8(\alpha-1)+4, \quad \text { para algum } \alpha \geq 2, \\
& v\left(x^{\alpha} d y\right)+1=v_{1}+4 \alpha \\
& v\left(x^{\left[\frac{v_{1}}{4}\right]+\beta} d y\right)+1=v_{1}+4\left[\frac{v_{1}}{4}\right]+4 \beta, \\
& v\left(x^{\beta} y d y\right)+1=2 v_{1}+4 \beta, \\
& v\left(\Theta_{2} \Omega_{1}\right)+1=\lambda+8(\alpha-1)+4, \quad \text { para algum } \alpha \geq 2
\end{aligned}
$$

Lembremos que pela condição (6.8) devemos ter

$$
v(\omega)>v\left(B_{2}\right)+\lambda+v_{1}+4-4\left[\frac{v_{1}}{4}\right]>8(\alpha-1)+\lambda+4 \geq \lambda+12
$$

Assim, devemos ter $g_{1}=0$ e $h_{1}=\frac{v_{1}}{4} b_{1}$. Ou seja,

$$
\begin{aligned}
& \omega=h_{1} x \Omega_{1}+\left(\sum_{\gamma=2}^{\left[\frac{v_{1}}{4}\right]}\left(g_{\gamma} x^{\left[\frac{v_{1}}{4}\right]+\gamma}+h_{\gamma} y x^{\gamma}\right)+\right. \\
& +\left\{\begin{array}{cc}
h y^{2}+\sum_{\delta=1}^{\left[\frac{v_{1}}{4}\right]}\left(l_{\delta} x^{2\left[\frac{v_{1}}{4}\right]+\delta}+m_{\delta} y x^{\left[\frac{v_{1}}{4}\right]+\delta}+\right. & \text { se } v_{1} \equiv 1 \bmod 4 \\
& \left.\left.+n_{\delta} y^{2} x^{\delta}\right)\right) d x- \\
g x^{2\left[\frac{v_{1}}{4}\right]+1}+h y^{2}+\sum_{\delta=1}^{\left[\frac{v_{1}}{4}\right]}\left(l_{\delta} y x^{\left[\frac{v_{1}}{4}\right]+\delta}+m_{\delta} x^{2\left[\frac{v_{1}}{4}\right]+1+\delta}+\right. & \text { se } v_{1} \equiv 3 \bmod 4 \\
\left.\left.+n_{\delta} y^{2} x^{\delta}\right)\right) d x- &
\end{array}\right. \\
& -\left(\sum_{\alpha=3}^{\left[\frac{v_{1}}{4}\right]-1} b_{\alpha-1} x^{\alpha}+\sum_{\beta=0}^{\left[\frac{v_{1}}{4}\right]}\left(c_{\beta} x^{\left[\frac{v_{1}}{4}\right]+\beta}+d_{\beta} x^{\beta} y\right)\right) d y-
\end{aligned}
$$


$-\Theta_{1} y d x-\frac{v_{0}}{\lambda-v_{1}} \Theta_{2} \Omega_{1} \bmod t^{l-4}$.

Como $\max \left\{v\left(x \Omega_{1}\right), v\left(x^{\left[\frac{v_{1}}{4}\right]+2} d x\right)\right\}=\max \left\{\lambda+8,4\left[\frac{v_{1}}{4}\right]+4+8\right\}<\lambda+12$, segue que $g_{2}=h_{1}=0, \log 0 b_{1}=0$, que por sua vez implica que a ordem de $p$ suba e conseqüentemente as ordens de $B_{2}, \Theta_{1}$ e $\Theta_{2}$ também subam. Disto concluímos que $h_{2}=\frac{v_{1}}{4} b_{2}$ e $v(\omega)>v\left(B_{2}\right)+\lambda+v_{1}-4\left[\frac{v_{1}}{4}\right]+4 \geq \lambda+20$. Assim,

$$
\begin{aligned}
& \omega=h_{2} x^{2} \Omega_{1}+\left(\sum_{\gamma=3}^{\left[\frac{v_{1}}{4}\right]}\left(g_{\gamma} x^{\left.\frac{v_{1}}{4}\right]+\gamma}+h_{\gamma} y x^{\gamma}\right)+\right. \\
& +\left\{\begin{array}{cc}
h y^{2}+\sum_{\delta=1}^{\left[\frac{v_{1}}{4}\right]}\left(l_{\delta} x^{2\left[\frac{v_{1}}{4}\right]+\delta}+m_{\delta} y x:^{\left[\frac{v_{1}}{4}\right]+\delta}+\right. & \text { se } v_{1} \equiv 1 \bmod 4 \\
\left.\left.+n_{\delta} y^{2} x^{\delta}\right)\right) d x- & \text { se } v_{1} \equiv 3 \bmod 4 \\
g x^{2\left[\frac{v_{1}}{4}\right]+1}+h y^{2}+\sum_{\delta=1}^{\left[\frac{v_{1}}{4}\right]}\left(l_{\delta} y x x^{\left[\frac{v_{1}}{4}\right]+\delta}+m_{\delta} x^{2\left[\frac{v_{1}}{4}\right]+1+\delta}+\right. \\
\left.\left.+n_{\delta} y^{2} x^{\delta}\right)\right) d x-
\end{array}\right. \\
& -\left(\sum_{\alpha=4}^{\left[\frac{v_{1}}{4}\right]-1} b_{\alpha-1} x^{\alpha}+\sum_{\beta=0}^{\left[\frac{v_{1}}{4}\right]}\left(c_{\beta} x^{\left[\frac{v_{1}}{4}\right]+\beta}+d_{\beta} x^{\beta} y\right)\right) d y- \\
& -\Theta_{1} y d x-\frac{v_{0}}{\lambda-v_{1}} \Theta_{2} \Omega_{1} \quad \bmod t^{l-4} \text {. }
\end{aligned}
$$

Usando o mesmo argumento acima teremos $h_{2}=g_{3}=0$, o que implica $b_{2}=0$ e que aumentamos a ordem de $p$. Consequientemente as ordens de $B_{2}, \Theta_{1}$ e $\Theta_{2}$ também aumentam. Concluímos ainda que $h_{3}=\frac{v_{1}}{4} b_{3}$ e que' $v\left(B_{2}\right)+\lambda+v_{1}-4\left[\frac{v_{1}}{4}\right]+4 \geq \lambda+28$.

Repetindo esse raciocínio podemos concluir que se a desigualdade em (6.8) ocorre, a ordem de $B_{2}$ é suficientemente grande de modo que a ordem de $\Upsilon$ supere $l-4$, o que garante o resultado, como no caso anterior.

$$
\text { Caso: } \lambda=v_{2}+v_{1}-4 m_{1}
$$

Antes de mais nada, observe que se definimos por $C=\sum_{\alpha=2}^{\left[\frac{v_{1}}{4}\right]-1} b_{\alpha-1} x^{\alpha-1}$ e $D=\sum_{\beta=0}^{\left[\frac{v_{2}-v_{1}}{4}\right]}\left(c_{\beta} x^{\left[\frac{v_{1}}{4}\right]+\beta-1}+d_{\beta} x^{\beta-1} y\right)$, temos para todo $k \geq 2$ que

$$
\left(\frac{p}{t^{4}}\right)^{k}=(C+D)^{k}=\sum_{i=0}^{k}\left(\begin{array}{c}
k \\
i
\end{array}\right) C^{k-i} D^{i}
$$


Obviamente as duas primeiras parcelas da última expressão são elementos de $\mathcal{O}$. Em $D^{i} \operatorname{com} i \geq 2$, a única parcela que não pertence ao auel local $\mathcal{O}$ é o termo da forma $\frac{y^{i}}{x^{i}}$. Como $i \geq 2$, escrevemos $i=2 r+s \operatorname{com} r \geq 1$ e $0 \leq s<2$. Assim, usando a expressão $h_{2}=y^{2}-x^{m_{1}}$, dada pela Proposição $2 . \overline{1}$, temos que $\left\{x, y, h_{2}\right\}$ é uma Base Standard Mínima para $\mathcal{O}$. Podemos então escrever

$$
\frac{y^{i}}{x^{i}}=\frac{y^{2 r} y^{s}}{x^{i}}=\frac{\left(x^{m_{1}}+h_{2}\right)^{r} y^{s}}{x^{i}}=\left(x^{r m_{1}-i}+\frac{\sum_{u=1}^{r}\left(\begin{array}{l}
r \\
u
\end{array}\right) x^{(r-u) m_{1}} h_{2}^{u}}{x^{i}}\right) y^{s} .
$$

Observe agora que a ordem de cada parcela do somatório acima é

$$
\begin{aligned}
4\left((r-u) m_{1}-i\right)+u v_{2} & =4\left(r\left(m_{1}-2\right)-u m_{1}-s\right)+u v_{2}= \\
& =u\left(v_{2}-8\right)+(r-u)\left(2 v_{1}-8\right)-4 s \geq 1 \\
& \geq v_{2}-8-4 s \geq v_{2}-12>l-v_{1}-v_{0} .
\end{aligned}
$$

Mostramos assim, que existe $B_{k} \in \mathcal{O}$ tal que $B_{k}=(C+$ $D)^{k} \bmod t^{l-v_{1}-v_{0}+1}$ para todo $k \geq 2$.

Utilizando o fato acima e as expressões de $p$ e $q$, para este caso, podemos escrever

$$
\begin{aligned}
\omega= & \left(\sum_{\gamma=1}^{\left[\frac{v_{2}-v_{1}}{4}\right]}\left(g_{\gamma} x^{\left[\frac{v_{1}}{4}\right]+\gamma}+h_{\gamma} x^{\gamma} y\right)\right) d x- \\
& -\left(\sum_{\alpha=2}^{\left[\frac{v_{1}}{4}\right]-1} b_{\alpha-1} x^{\alpha}+\sum_{\beta=0}^{\left[\frac{v_{2}-v_{1}}{4}\right]}\left(c_{\beta} x^{\left[\frac{v_{1}}{4}\right]+\beta}+d_{\beta} x^{\beta} y\right)\right) d y- \\
& -\Theta_{1} y d x-\frac{v_{0}}{\lambda-v_{1}} \Theta_{2} \Omega_{1} \bmod t^{l-v_{0}+1},
\end{aligned}
$$

onde

$$
\begin{aligned}
\Theta_{1} & =\sum_{k=2}^{\infty} B_{k}= \\
& =\sum_{k=2}^{\infty}\left(\frac{v_{1}}{4}\right)\left(\sum_{\alpha=2}^{\left[\frac{v_{1}}{4}\right]-1} b_{\alpha-1} x^{\alpha-1}+\sum_{\beta=0}^{\left[\frac{v_{2}-v_{1}}{4}\right]}\left(c_{\beta} x^{\left[\frac{v_{1}}{4}\right]+\beta-1}+d_{\beta} x^{\beta-1} y\right)\right)^{k}
\end{aligned}
$$

e

$$
\Theta_{2}=\sum_{k=2}^{\infty}\left(\left(\begin{array}{l}
\frac{\lambda}{4} \\
k
\end{array}\right)-\left(\begin{array}{c}
\frac{v_{1}}{4} \\
k
\end{array}\right)\right) B_{k}=
$$

\footnotetext{
${ }^{1}$ lembre que $m_{1} v_{0}=n_{1} v_{1}$ e que neste caso $v_{0}=4$ e $n_{1}=n_{2}=2$.
} 


$$
\begin{aligned}
=\sum_{k=2}^{\infty}\left(\left(\begin{array}{c}
\frac{\lambda}{\alpha} \\
k
\end{array}\right)-\left(\begin{array}{c}
\frac{v_{1}}{4} \\
k
\end{array}\right)\right)\left(\sum_{\alpha=2}^{\left[\frac{v_{1}}{4}\right]-1} b_{\alpha-1} x^{\alpha-1}+\right. \\
\left.+\sum_{\beta=0}^{\left[\frac{v_{2}-v_{1}}{4}\right]}\left(c_{\beta} x^{\left[\frac{v_{1}}{4}\right]+\beta-1}+d_{\beta} x^{\beta-1} y\right)\right)^{k},
\end{aligned}
$$

$\operatorname{com} v\left(\Theta_{1}\right)=v\left(\Theta_{2}\right)=8(\alpha-1)$ para algum $\alpha \geq 2$. Assim,

$$
v\left(\Theta_{1} y d x\right)+1=8(\alpha-1)+v_{1}+4<8(\alpha-1)+\lambda+4=v\left(\Theta_{2} \Omega_{1}\right)+1
$$

Note que a condição (6.8) para esta forma normal é

$$
v(\omega)>2 v(p)-4+v_{2}-4\left[\frac{v_{1}}{4}\right]>2 v(p)+v_{2}-v_{1}-4=\lambda+8 \alpha-4
$$

Como em $\omega$ temos

$$
\begin{aligned}
& v\left(x^{\left[\frac{v_{1}}{4}\right]+\gamma} d x\right)+1=4 \gamma+4+4\left[\frac{v_{1}}{4}\right] \\
& v\left(x^{\gamma} y d x\right)+1=4 \gamma+4+v_{1} \\
& v\left(x^{\alpha} d y\right)+1=4 \alpha+v_{1}
\end{aligned}
$$

e como $2 v(p)-4+v_{2}-4\left[\frac{v_{1}}{4}\right]>\lambda+12$, devemos ter $g_{1}=0$ e $h_{1}=\frac{v_{1}}{v_{0}} b_{1}$, ou seja,

$$
\begin{aligned}
\omega= & h_{1} x \Omega_{1}+\left(\sum_{\gamma=2}^{\left[\frac{v_{2}-v_{1}}{4}\right]} g_{\gamma} x^{\left[\frac{v_{1}}{4}\right]+\gamma}+h_{\gamma} x^{\gamma} y\right) d x- \\
& -\left(\sum_{\alpha=3}^{\left[\frac{v_{1}}{4}\right]-1} b_{\alpha-1} x^{\alpha}+\sum_{\beta=0}^{\left[\frac{v_{2}-v_{1}}{4}\right]} c_{\beta} x^{\left[\frac{v_{1}}{4}\right]+\beta}+d_{\beta} x^{\beta} y\right) d y+ \\
& -\Theta_{1} y d x-\frac{v_{0}}{\lambda-v_{1}} \Theta_{2} \Omega_{1} \bmod t^{l-v_{0}+1} .
\end{aligned}
$$

Novamente, uma análise sobre as ordens dos termos envolvidos, leva-nos a concluir que $g_{2}=h_{1}=0$ e $h_{2}=\frac{v_{1}}{v_{0}} b_{2}$, mas dessa forma teremos $b_{1}=0$, $v\left(\Theta_{1} y d x\right)+1=20+v_{1}<\lambda+20=v\left(\Theta_{2} \Omega_{1}\right)+1$ e $2 v(p)-4+v_{2}-4\left[\frac{v_{1}}{4}\right]>\lambda+20$.

Repetindo sucessivamente o processo acima descrito, concluimos que $v(\Upsilon)>l-4$ e portanto, $A(t)=0$.

A fim de ilustrar a dificuldade em identificar ramos equivalentes, quando esses não se encontram na forma normal, apresentamos o exemplo abaixo. 
Exemplo 6.1 Sejam

$$
\begin{aligned}
& C_{1}:\left\{\begin{array}{l}
x=t^{4} \\
y=t^{9}+t^{10}+\frac{19}{18} t^{11}+t^{15}
\end{array}\right. \\
& C_{2}:\left\{\begin{array}{l}
x=t^{4} \\
y=t^{9}+t^{10}+\frac{19}{18} t^{11}+2 t^{15}
\end{array}\right. \\
& C_{3}:\left\{\begin{array}{l}
x=t^{4} \\
y=t^{9}+3 t^{10}+\frac{19}{2} t^{11}+243 t^{14}+2268 t^{15}+ \\
+137781 t^{17}+413343 t^{18}+1869885 t^{19}
\end{array}\right.
\end{aligned}
$$

O que dizer sobre os ramos acịma, no que diz respeito à equivalência?

Antes da apresentação das tabelas deste capítulo, nossa intuição levaria a afirmar erroneamente que $C_{1} \sim C_{2}$. Como os ramos $C_{1}$ e $C_{2}$ possuem $\lambda=10=2 v_{1}-4 j, a_{i}=0$ para todo $i=1, \ldots,\left[\frac{v_{1}}{4}\right]-j$ e $a_{[}\left[\frac{v_{1}}{4}\right]-j+1=\frac{v_{1}+\lambda}{2 v_{1}}=\frac{19}{18}$. Consultando a tabela para $\Gamma=\left\langle 4, v_{1}\right\rangle$, vemos que $C_{1}$ e $C_{2}$ estão na forma normal. Assim, teríamos que $C_{1} \sim C_{2}$ se, e somente se, houvesse $c \in K^{*}$ com $a_{i}=c^{i-v_{1}} b_{i}$ para todos os coeficientes $a_{i}$ de $C_{1}$ e $b_{i}$ de $C_{2}$ de $t^{i}$, com $i>v_{1}$.

Como $a_{\lambda}=1=c=c^{\lambda-v_{1}} b_{\lambda}$ e $a_{15}=1 \neq 2=c^{15-v_{1}} b_{15}$, temos que $C_{1} \not C_{2}$.

Mas o que dizer de $C_{3}$ com relação a $C_{1}$ e $C_{2}$ ?

Observe que $C_{3}$ não está na forma normal.

Para obter a forma normal de $C_{3}$ considere inicialmente a homotetia

$$
\begin{array}{r}
t_{1}=3 t \\
y_{1}=3 y,
\end{array}
$$

donde obtemos

$$
\left\{\begin{array}{l}
x_{1}=t_{1}^{4} \\
y_{1}=t_{1}^{9}+t_{1}^{10}+\frac{19}{18} t_{1}^{11}+t_{1}^{14}+\frac{28}{9} t_{1}^{15}+21 t_{1}^{17}+21 t_{1}^{18}+\frac{95}{3} t_{1}^{19}
\end{array}\right.
$$

Uma vez que $14=v\left(\frac{x \Omega_{1}}{d x}\right)$, onde $\Omega_{1}=x d y-\frac{9}{4} y d x$, podemos eliminar $o$ termo $t_{1}^{14}$. Para isso, usemos a mudança

$$
t_{2}=t_{1}\left(1+\frac{4 x_{1}^{2}}{t_{1}^{4}}\right)^{\frac{1}{4}}
$$




$$
y_{2}=y_{1}+9 x_{1} y_{1}
$$

que nos fornece

$$
\left\{\begin{array}{l}
x_{2}=t_{2}^{4} \\
y_{2}=t_{2}^{9}+t_{2}^{10}+\frac{19}{18} t_{2}^{11}+t_{2}^{14}+\frac{28}{9} t_{2}^{15}+21 t_{2}^{17}+21 t_{2}^{18}+\frac{95}{3} t_{2}^{19}+A\left(t_{1}\right),
\end{array}\right.
$$

com

$$
A\left(t_{1}\right)=\frac{-4 x_{1}^{2} d y_{1}+9 x_{1} y_{1} d x_{1}}{d x_{1}}-t_{1}^{9}\left(\frac{9}{4}\right)\left(\frac{4 x_{1}^{2}}{2}\right)^{2}-t_{1}^{10}\left(\frac{10}{\frac{1}{4}} \frac{4}{2}\right)\left(\frac{4 x_{1}^{2}}{t_{1}^{1}}\right)^{2}
$$

$\bmod t_{1}^{20}$

$$
=-t_{1}^{14}-\frac{19}{9} t_{1}^{15}-21 t_{1}^{17} i_{i}^{i}-35 t_{1}^{18} \bmod t_{1}^{20} .
$$

Note que

$$
-\frac{45}{2}
$$

$$
t_{2}^{i}=t_{1}^{i}\left(1+\frac{4 x_{1}^{2}}{t_{1}^{4}}\right)^{\frac{i}{4}}=t_{1}^{i}\left(1+4 t_{1}^{4}\right)^{\frac{i}{4}}=t_{1}^{i}+i t_{1}^{i+4} \quad \bmod t_{1}^{i+8} .
$$

Assim, escrevendo $\frac{28}{9} t_{2}^{15}=t_{2}^{15}+\frac{19}{9} t_{2}^{15} e$

$$
t_{2}^{14}+\frac{19}{9} t_{2}^{15}+21 t_{2}^{17}+21 t_{2}^{18}+\frac{95}{3} t_{2}^{19}=t_{1}^{14}+\frac{19}{9} t_{1}^{15}+21 t_{1}^{17}+35 t_{1}^{18} \cdot \bmod t_{1}^{20},
$$

temos

$$
y_{2}=t_{2}^{9}+t_{2}^{10}+\frac{19}{18} t_{2}^{11}+t_{2}^{15} \bmod t_{2}^{20}
$$

Uma vez que todo inteiro maior ou igual a 20 pertence ao conjunto $\Gamma U$ $\left(\Gamma+v_{1}-v_{0}\right)$, podemos eliminar tais termos sem que alteremos os termos de ordem inferior, seguindo assim que $C_{3}$ é equivalente à $C_{1}$.

\subsection{Ramos com Multiplicidade 5}

A análise geral dos ramos com $v_{0}=5$ seria bem mais trabalhosa do que o caso $v_{0}=4$, pois teríamos muitas possibilidades a analisar. Como no Exemplo 5.4 fizemos o estudo completo das classes de equidiferenciabilidades para os ramos com semigrupo $\Gamma=\langle 5,11\rangle$, escolhemos o valor 11 como limitante para $v_{1}$. 
O Exemplo 5.3, nos indica.que os ramos com semigrupo $\Gamma=\left\langle 5, v_{1}\right\rangle$, onde $v_{1}<10$, admitem apenas uma DNEM. Assim, usando o Teorema 4.1, o Corolário 5.1 e uma homotetia, temos as seguintes possibilidades:

Se $\lambda=4 v_{1}-5 j$, então podemos representar cada classe de equivalência por um ramo com parametrização da forma

$$
\left\{\begin{array}{l}
x=t^{5} \\
y=t^{v_{1}}+t^{\lambda}+\sum_{i=1}^{j-\left[\frac{v_{1}}{5}\right]-1} a_{i} t^{3 v_{1}-5\left(j-\left[\frac{v_{1}}{5}\right]+1-i\right)},
\end{array}\right.
$$

onde $\Lambda \backslash \Gamma=\left\{4 v_{1}-5 k ; k=1, \ldots, j-1\right\}$.

Se $\lambda=3 v_{1}-5 j$, então um representante para cada classe de equivalência, pode ser dado por

$$
\left\{\begin{array}{l}
x=t^{5} \\
y=t^{v_{1}}+t^{\lambda}+\sum_{i=1}^{\left[\frac{v_{1}}{5}\right]} a_{i} t^{4 v_{1}-5\left(\left[\frac{v_{1}}{5}\right]+j+1-i\right)},
\end{array}\right.
$$

e $\Lambda \backslash \Gamma=\left\{3 v_{1}-5 k, 4 v_{1}-5 k ; k=1, \ldots, j-1\right\}$.

Na verdade, mesmo para ramos com semigrupo $\Gamma=\langle 5,11\rangle$ e $\lambda=4 v_{1}-5 j$ ou $\lambda=3 v_{1}-5 j$, salvo o caso $\lambda=23$, conforme o Exemplo 5.4 indica, temos as formas normais acima.

Se $\lambda=23$ e $a \neq 0$ em (5.2), a mudança de coordenadas

$$
\begin{aligned}
& t_{1}=t\left(1+\frac{b\left(55 x^{2}-60 y^{2}\right)}{143 a t^{5}}\right)^{\frac{1}{5}} \\
& y_{1}=y+\frac{11 b}{13 a} x y+\frac{33 b^{2}}{169 a^{2}} x^{2} y+\frac{11 b^{3}}{2197 a^{3}} x^{3} y
\end{aligned}
$$

em (5.2) elimina o termo $b t_{1}^{29}$ sem alterar os termos de ordem inferior. Como todos os termos de ordem superior a 29 se enquandram no Teorema 4.1, podemos considerar que cada representante de uma classe de equivalência com semigrupo $\Gamma=\langle 5,11\rangle$ e invariante $\lambda$ de Zariski igual 23 pode ser dado por

se $a \neq 0$ ou por

$$
\left\{\begin{array}{l}
x=t^{5} \\
y=t^{11}+t^{23}+a t^{24}
\end{array}\right.
$$

$$
\left\{\begin{array}{l}
x=t^{5} \\
y=t^{11}+t^{23}+b t^{29}
\end{array}\right.
$$

Para os demais casos, i.e., quando $\lambda=12$, vamos indicar os passos que realizamos para proceder à eliminação dos parâmetros em (5.3), correspondentes aos termos com ordem $v(\Omega)-v_{0}+\gamma \operatorname{com} \gamma \in \Gamma$ e $\Omega$ uma DNEM com 
ordem maior que a ordem de $\Omega_{1}=x d y-\frac{11}{5} y d x$, sem alterar os termos de ordem inferior. Não apresentamos explicitamente os cálculos envolvidos por serem extensos e repetitivos. No que segue usaremos as notações usadas no Exemplo 5.4.

Inicialmente escolha um termo da forma $a_{l} t^{l}$ na parametrização em (5.3), com $l=v(\Omega)-5+\gamma$, onde $\gamma \in \Gamma$ e $\Omega$ uma DNEM com $v(\Omega)+1>\lambda+5=$ $v\left(\Omega_{1}\right)+1$.

Escolla $h \in \mathcal{O} \operatorname{com} v(h)=\gamma$ e escreva $h \Omega=q_{l} d x-p_{l} d y$. Note que $v\left(p_{l}\right)=5+\delta$ com $\delta \in \Gamma$ para toda $\Omega$ no Exemplo 5.4 .

Se $l<2 v\left(p_{l}\right)-2 v_{0}+v_{1}=2 v\left(p_{l}\right)+1$, então o Lema 4.1 garante que a mudança de coordenadas

$$
\begin{aligned}
& t_{1}=t\left(1+\frac{\alpha_{l} p_{l}}{t^{5}}\right)^{\frac{1}{5}} \\
& y_{1}=y+\alpha_{l} q_{l}
\end{aligned}
$$

permite eliminar o termo $a_{l} t^{\prime}$ da parametrização (5.3), para alguma escolha conveniente de $\alpha_{l}$, sem que alteremos os temos de ordem inferior.

Se $l \geq 2 v\left(p_{l}\right)-2 v_{0}+v_{1}=2 v\left(p_{l}\right)+1=2(5+\delta)+1=\delta+11 \in \Gamma$, então para cada valor de $\theta \in v\left(\frac{\mathcal{O d O}}{d x}\right)$ com $2 v\left(p_{l}\right)+1 \leq \theta<l$ escolha uma $\Omega_{\theta}=q_{\theta} d x-p_{\theta} d y, \operatorname{com} v\left(\frac{\Omega_{\theta}}{d x}\right)=\theta$ e considere a mudança de coordenadas

$$
\begin{aligned}
& t_{1}=t\left(1+\frac{P}{t^{5}}\right)^{\frac{1}{5}} \\
& y_{1}=y+Q
\end{aligned}
$$

$\operatorname{com} P=\sum_{\theta=2 v\left(p_{l}\right)+1}^{l} \alpha_{\theta} p_{\theta}$ e $Q=\sum_{\theta=2 v\left(p_{l}\right)+1}^{l} \alpha_{\theta} q_{\theta}$.

Ao analisar a série $y_{1}$, veremos que existe uma escollha para $\alpha_{\theta} \operatorname{com} \theta=$ $2 v\left(p_{l}\right)+1, \ldots, l$ que elimina o termo $a_{l} t_{1}^{l}$ sem que alteremos os termos de ordem inferior.

Procedendo como indicado acima, apartir das potências de mais baixa ordem, podemos obter as seguintes formas normais para os ramos com semigrupo $\Gamma=\langle 5,11\rangle$ e $\lambda=12$ :

- Se $a \neq \frac{23}{22}$ e $b \neq \frac{(2 a+1)(2 a-1)}{3}$, então $\Lambda \backslash \Gamma=\{17,23,28,29,34,39\}$ e podemos considerar

$$
\left\{\begin{array}{l}
x=t^{5} \\
y=t^{11}+t^{12}+a t^{13}+b t^{14}+d t^{19}
\end{array}\right.
$$


- Se $a \neq \frac{23}{22}$ e $b=\frac{(2 n+1)(2 a-1)}{3}$, temos $\Lambda \backslash \Gamma=\{17,23,28,34,39\}$ e basta nos restringirmos a

$$
\left\{\begin{array}{l}
x=t^{5} \\
y=t^{11}+t^{12}+a t^{13}+\frac{(2 a+1)(2 a-1)}{3} t^{14}+d t^{19}+e t^{24}
\end{array}\right.
$$

- Caso $a=\frac{23}{22}$ e $b \neq \frac{136}{121}$, teremos $\Lambda \backslash \Gamma=\{17,24,28,29,34,39\}$ e a forma normal

$$
\left\{\begin{array}{l}
x=t^{5} \\
y=t^{11}+t^{12}+\frac{23}{22} t^{13}+b t^{14}+c t^{18}
\end{array}\right.
$$

- Se $a=\frac{23}{22}, b=\frac{136}{121}$ e $A \neq 0$ (Veja Exemplo 5.4), o conjunto das lacunas especiais é $\Lambda \backslash \Gamma=\{17,28,29,34,39\}$ e temos

$$
\left\{\begin{array}{l}
x=t^{5} \\
y=t^{11}+t^{12}+\frac{23}{22} t^{13}+\frac{136}{121} t^{14}+c t^{18}+d t^{19}
\end{array}\right.
$$

- Quando $a=\frac{23}{22}, b=\frac{136}{121}, A=0$ e $B \neq 0$ (Veja Exemplo 5.4), teremos $\Lambda \backslash \Gamma=\{17,28,34,39\}$ e a forma normal

$$
\left\{\begin{array}{l}
x=t^{5} \\
y=t^{11}+t^{12}+\frac{23}{22} t^{13}+\frac{136}{121} t^{14}+c t^{18}+d t^{19}+e t^{24}
\end{array}\right.
$$

- Se $a=\frac{23}{22}, b=\frac{136}{12 !}, A=0$ e $B=0$ (Veja Exemplo 5.4), então $\Lambda \backslash \Gamma=$ $\{17,28,39\}$ e teinos

$$
\left\{\begin{array}{l}
x=t^{5} \\
y=t^{11}+t^{12}+\frac{23}{22} t^{13}+\frac{136}{121} t^{14}+c t^{18}+d t^{19}+e t^{24}+f t^{3429}
\end{array}\right.
$$

Por estar $v_{1}$ limitado, ou seja, $v_{1} \in\{6,7,8,9,11\}$, pode-se mostrar que dois ramos com o mesmo conjunto $\Lambda \backslash \Gamma$ e que são dados pelas formas normais acima são equivalentes se, e somente se, diferem por uma homotetia. Não explicitaremos os cálculos feitos, mas indicaremos o roteiro que seguimos para efetuá-los.

Inicialmente, note que por analogia ao caso $v_{0} \leq 4, l$ é a lacuna limitante de $\Lambda$, então todos os termos com ordem maior do que $l-5$ podem ser eliminados, como indicamos anteriormente, de modo a deixar inalterados os termos de ordem inferior. 
Para cada semigrupo $\Gamma=\left\langle 5, v_{1}\right\rangle$ e forma normal fixados, tome a expressão genérica de funções $p, q \in \mathcal{O}$, de modo que $5<v(p)<l-5$ e $v_{1}<v(q)<l-5$, i.e.,

$$
p=\sum_{\alpha=\left(\alpha_{1}, \alpha_{2}\right) \in \mathrm{N}^{2}} c_{\alpha} x^{\alpha_{1}} y^{\alpha_{2}}
$$

e

$$
q=\sum_{\delta=\left(\delta_{1}, \delta_{2}\right) \in \mathrm{N}^{2}} d_{\delta} x^{\delta_{1}} y^{\delta_{2}}
$$

$\operatorname{com} \alpha_{2}<5$ e $\delta_{2}<5$.

Agora considere a mudança de coordenadas

$$
\begin{aligned}
& t_{1}=t\left(1+\frac{p}{t^{5}}\right)^{\frac{1}{5}} \\
& y_{1}=y+q
\end{aligned}
$$

Ao analisar a série $y_{1}$, coustataremos que as escollias dos parâmetros $c_{c k}$ e $d_{\delta}$ a serem feitas de modo a não iutroduzir termos estraulıos à forma norınal, acabam por fixar todos os termos de ordens menores ou iguais a $l-5$, como os termos de ordem superior são irrelevantes, concluímos que qualquer mudança de coordenadas da forma (6.9) que mantém a forma normal conșiderada, age como a identidade. Assim, as únicas mudanças de coordenadas não triviais são as homotetias.

Dessa forma, classificamos todos os ramos com $v_{0} \leq 4$ e aqueles com $v_{0}=5$ e $v_{1} \leq 11$. A saber, tais ramos podem ser unicamente representados, a menos de homotetias, como nas tabelas que apresentamos no final deste. capítulo, onde omitimos os ramos com $v_{0}=1$, uma vez que todos esses são equivalentes. 


\subsection{Tabelas}

\begin{tabular}{|c|c|}
\hline \multicolumn{2}{|c|}{$\Gamma=\left\langle 2, v_{1}\right\rangle$} \\
\hline$\left\{\begin{array}{l}x=t^{2} \\
y=t^{v_{1}}\end{array}\right.$ & $\Lambda \backslash \Gamma$ \\
\hline
\end{tabular}

\begin{tabular}{|c|c|}
\hline \multicolumn{2}{|c|}{$\Gamma=\left\langle 3, v_{1}\right\rangle$} \\
\hline Forma Normal & $\Lambda \backslash \Gamma$ \\
\hline$\left\{\begin{array}{l}x=t^{3} \\
y=t^{v_{1}}\end{array}\right.$ & \{\} \\
\hline$\left\{\begin{array}{l}x=t^{3} \\
y=t^{v_{1}}+t^{2 v_{1}-3 j}\end{array}\right.$ & $\begin{array}{c}2 v_{1}-3 s \\
\end{array}$
\end{tabular}

\begin{tabular}{|c|c|}
\hline \multicolumn{2}{|l|}{$\Gamma=\left\langle 4, v_{1}\right\rangle$} \\
\hline Forma Normal & $\Lambda \backslash \Gamma$ \\
\hline$\left\{\begin{array}{l}x=t^{4} \\
y=t^{v_{1}}\end{array}\right.$ & \{\} \\
\hline $\begin{aligned} x= & t^{4} \\
y= & t^{v_{1}}+t^{3 v_{1}-4 j}+ \\
& +\sum_{i=2}^{j-\left[\frac{v_{1}}{4}\right]-1} a_{i} t^{2 v_{1}-4\left(j-\left[\frac{v_{1}}{4}\right]+1-i\right)}\end{aligned}$ & $\begin{array}{c}3 v_{1}-4 s \\
1 \leq s \leq j-1\end{array}$ \\
\hline$\left\{\begin{array}{l}x=t^{4} \\
y=t^{v_{1}}+t^{2 v_{1}-4 j}+ \\
\quad+\sum_{i=k}^{j-2+k} a_{i} t^{3 v_{1}-4}\left(\left[\frac{v_{1}}{4}\right]+j+1-i\right) \\
\quad 1 \leq k \leq\left[\frac{v_{1}}{4}\right]-j\end{array}\right.$ & $\begin{array}{c}2 v_{1}-4 s \\
1 \leq s \leq j-1 \\
3 v_{1}-4 s \\
1 \leq s \leq\left[\frac{v_{1}}{4}\right]+1-k\end{array}$ \\
\hline $\begin{aligned} x= & t^{4} \\
y= & t^{v_{1}}+t^{2 v_{1}-4 j}+ \\
& +\sum_{i=\left[\frac{v_{1}}{4}\right]-1}^{\left[\frac{v_{1}}{4}\right]-j+1} a_{i} t^{3 v_{1}-4}\left(\left[\frac{v_{1}}{4}\right]+j+1-i\right) \\
& a_{\left[\frac{v_{1}}{4}\right]-j+1} \neq \frac{v_{1}+\lambda}{2 v_{1}}\end{aligned}$ & $\begin{array}{c}2 v_{1}-4 s \\
1 \leq s \leq j-1 \\
3 v_{1}-4 s \\
1 \leq s \leq\left[\frac{v_{1}}{4}\right]+1-j\end{array}$ \\
\hline $\begin{aligned} x= & t^{4} \\
y= & t^{v_{1}}+t^{2 v_{1}-4 j}+ \\
& +\sum_{i=\left[\frac{v_{1}}{4}\right]-j+1}^{\left[v_{1}\right.} a_{i} t^{3 v_{1}-4}\left(\left[\frac{v_{1}}{4}\right]+j+1-i\right) \\
& a_{\left[\frac{v_{1}}{4}\right]-j+1}=\frac{v_{1}+\lambda}{2 v_{1}}\end{aligned}$ & $\begin{array}{c}2 v_{1}-4 s \\
3 v_{1}-4 s \\
1 \leq s \leq j-1\end{array}$ \\
\hline
\end{tabular}




\begin{tabular}{|c|c|}
\hline \multicolumn{2}{|c|}{$\Gamma=\left\langle 4, v_{1}, v_{2}\right\rangle$} \\
\hline Forma Normal & $\Lambda \backslash \Gamma$ \\
\hline$\left\{\begin{array}{c}x=t^{4} \\
y=t^{v_{1}}+t^{v_{2}+v_{1}-4 m_{1}}+ \\
+\sum_{i=2}^{\left[\frac{v_{1}}{4}\right]} a_{i} t^{v_{2}-4}\left(\left[\frac{v_{1}}{4}\right]+2-i\right)\end{array}\right.$ & $\begin{array}{c}v_{2}+v_{1}-4 s \\
\end{array}$
\end{tabular}

\begin{tabular}{|c|c|}
\hline \multicolumn{2}{|c|}{$\Gamma=\left\langle 5, v_{1}\right\rangle v_{1} \leq 11 \mathbf{e} \lambda \neq 12$} \\
\hline Forma Normal & $\Lambda \backslash \Gamma$ \\
\hline$\left\{\begin{array}{l}x=t^{5} \\
y=t^{v_{1}}\end{array}\right.$ & \{\} \\
\hline $\begin{aligned} x= & t^{5} \\
y= & t^{v_{1}}+t^{4 v_{1}-5 j}+ \\
& +\sum_{i=1}^{j-\left[\frac{v_{1}}{5}\right]-1} a_{i} t^{3 v_{1}-5\left(j-\left[\frac{v_{1}}{5}\right]+1-i\right)}\end{aligned}$ & $\begin{array}{c}4 v_{1}-5 s \\
1 \leq s \leq j-1\end{array}$ \\
\hline$\left\{\begin{aligned} x= & t^{5} \\
y= & t^{v_{1}}+t^{3 v_{1}-5 j}+ \\
& +\sum_{i=1}^{\left[\frac{v_{1}}{5}\right]} a_{i} t^{4 v_{1}-5\left(\left[\frac{v_{1}}{5}\right]+j+1-i\right)} \\
& j \neq 2 \text { se } v_{1}=11\end{aligned}\right.$ & $\begin{array}{c}3 v_{1}-5 s \\
4 v_{1}-5 s \\
1 \leq s \leq j-1\end{array}$ \\
\hline $\begin{array}{l}x=t^{5} \\
y=t^{11}+t^{23}+a t^{24} \\
\quad a \neq 0\end{array}$ & $28,34,39$ \\
\hline $\begin{array}{l}x=t^{5} \\
y=t^{11}+t^{23}+b t^{29}\end{array}$ & 28,39 \\
\hline
\end{tabular}




\begin{tabular}{|c|c|}
\hline$\Gamma=\langle 5,11\rangle \lambda$ & \\
\hline Forma Normal & $\Lambda \backslash \Gamma$ \\
\hline $\begin{aligned} x= & t^{5} \\
y= & t^{11}+t^{12}+a t^{13}+b t^{14}+d t^{19} \\
& a \neq \frac{23}{22} \text { e } b \neq \frac{(2 a+1)(2 a-1)}{3}\end{aligned}$ & $17,23,28,29,34,39$ \\
\hline$\left\{\begin{aligned} x= & t^{5} \\
y= & t^{11}+t^{12}+a t^{13}+b t^{14}+ \\
& +d t^{19}+e t^{24} \\
& \neq \frac{23}{22} \text { e } b=\frac{(2 a+1)(2 a-1)}{3}\end{aligned}\right.$ & $17,23,28,34,39$ \\
\hline $\begin{array}{c}x=t^{5} \\
y=t^{11}+t^{12}+\frac{23}{22} t^{13}+b t^{14}+c t^{18} \\
b \neq \frac{136}{121}\end{array}$ & $17,24,28,29,34,39$ \\
\hline$\left\{\begin{array}{c}x=t^{5} \\
y=t^{11}+t^{12}+\frac{23}{22} t^{13}+ \\
+\frac{136}{121} t^{14}+c t^{18}+d t^{19} \\
A \neq 0\end{array}\right.$ & $17,28,29,34,39$ \\
\hline $\begin{array}{l}x=t^{5} \\
y=t^{11}+t^{12}+\frac{23}{22} t^{13}+ \\
+\frac{136}{121} t^{14}+c t^{18}+d t^{19}+e t^{24} \\
A=0 \\
B \neq 0\end{array}$ & $17,28,34,39$ \\
\hline$\left\{\begin{array}{c}x=t^{5} \\
y=t^{11}+t^{12}+\frac{23}{22} t^{13}+\frac{136}{121} t^{14} \\
+c t^{18}+d t^{19}+e t^{24}+f t^{34} \\
A=0 \\
B=0\end{array}\right.$ & $17,28,39$ \\
\hline
\end{tabular}

$$
\begin{gathered}
A=88 d-\frac{440215}{644204}-267 c \\
B=\frac{1807239518543737}{5379063454198368}-\frac{4174016}{71289} d^{2}+143 e+\frac{462825631}{379542636} d
\end{gathered}
$$

\subsection{Resposta à uma questão de Zariski}

Zariski dedica os parágrafos 4, 5 e 6 do Capítulo VI de [Z2], ao estudo dos ramos com semigrupo da forma $\Gamma=\left\langle v_{0}, v_{0}+1\right\rangle$. Dentre outras coisas, calcula a dimensão da componente genérica do espaço que parametriza todas as 
classes de equivalência dos ramos numa classe de equisingularidade dada por $\Gamma$ como acima (ou mais geralmente para $\left.\Gamma=\left\langle v_{0}, h v_{0}+1\right\rangle \operatorname{com} h \geq 1\right\rangle$. Tal resultado foi posteriormente estendido por Delorme, em [D2], para qualquer semigrupo do tipo $\Gamma=\left\langle v_{0}, v_{1}\right\rangle$.

Zariski deixa uma pergunta a respeito da classificação dos ramos com semigrupos da forma $\Gamma=\left\langle v_{0}, v_{0}+1\right\rangle$. Nesta seção responderemos tal pergunta, que para ser formulada necessita das consideraçōes que seguem.

Seja $\Gamma=\left\langle v_{0}, v_{1}\right\rangle=\left\langle v_{0}, v_{0}+1\right\rangle$ um semigrupo de um ramo. Para cada $s \in\left\{1, \ldots, v_{0}-2\right\}$ defina

$$
\begin{aligned}
& \mathcal{L}_{s}=\left\{s v_{0}+s+1, s v_{0}+s+2, \ldots, s v_{0}+s+v_{0}-1-s\right\}, \\
& \bar{s}=v_{0}-s-2 \quad \text { e } \quad s^{\prime}=\min \{s, \bar{s}\} .
\end{aligned}
$$

Observação 6.1 Note que as lacunas de $\Gamma$ maiores que $s\left(v_{0}+1\right)$ para $s \geq 1$, são da forma $i\left(v_{0}+1\right)-j v_{0}$ com $s+1 \leq i \leq v_{0}-1 e 1 \leq j \leq i-s$.

Deste modo, podemos interpretar $\mathcal{L}_{s}$ como o conjunto dos naturais consecutivos que são lacunas de $\Gamma$ maiores que $s\left(v_{0}+1\right)$ e menores que $(s+1) v_{0}$.

De fato, se $i\left(v_{0}+1\right)-j v_{0}$ é uma lacuna maior que $s\left(v_{0}+1\right)$, escrevemos

$$
i\left(v_{0}+1\right)-j v_{0}=s v_{0}+s+(i-s-j) v_{0}+k,
$$

onde $k=i-s$. Tomando $j=i-s$ e observando que $1 \leq k \leq v_{0}-s-1$, concluimos o fato.

Observe ainda que para todo $1 \leq s \leq v_{0}-2$, temos que

$$
\min \left(\mathcal{L}_{s}\right)=s v_{0}+s+1=(s+1)\left(v_{0}+1\right)-v_{0},
$$

i.e., $\min \left(\mathcal{L}_{s}\right) \in \Gamma+v_{1}-v_{0}$ para todo $1 \leq s \leq v_{0}-2$.

De acordo corn a Proposição 4.2 (ou o Exemplo 3.2) todo ramo com semigrupo $\Gamma$ é equivalente a um ramo da forma

$$
\left\{\begin{array}{l}
x=t^{v_{0}} \\
y=t^{v_{0}+1}+\sum_{i \in \cup_{s=1}^{v_{0}-2} \overline{\mathcal{C}}_{s}} a_{i} t^{i},
\end{array}\right.
$$

onde $\overline{\mathcal{L}}_{s}=\mathcal{L}_{s} \backslash\left\{\min \left(\mathcal{L}_{s}\right)\right\}$ e $1 \leq s \leq v_{0}-2$. Note que $\overline{\mathcal{L}}_{v_{0}-2}=\{\}$, pois $\mathcal{L}_{v_{0}-2}=\left\{\left(v_{0}-1\right)\left(v_{0}+1\right)-v_{0}\right\}$.

Com as notações anteriores, Zariski em [Z2], prova o seguinte teorema. 
Teorema 6.1. Fixado $s \in\left\{2, \ldots, v_{0}-3\right\}$ qualquer, existe um polinômio $R_{s} \in$ $\mathbb{Z}\left[a_{v_{0}+3}, \ldots, a_{v_{0}+s^{\prime}+1}\right]^{*}$, tal que todo ramo dado por

$$
\left\{\begin{array}{l}
x=t^{v_{0}} \\
y=t^{v_{0}+1}+a_{v_{0}+3} t^{v_{0}+3}+\cdots+a_{2 v_{0}-1} t^{2 v_{0}-1}+\sum_{\substack{i>2 v_{0}-1 \\
i \notin \Gamma}} a_{i} t^{i}
\end{array}\right.
$$

$\operatorname{com} R_{s}\left(a_{v_{0}+3}, \ldots, a_{v_{0}+s^{\prime}+1}\right) \neq 0$ é equivalente a um ramo do tipo

$$
\left\{\begin{array}{l}
x=t^{v_{0}} \\
y=t^{v_{0}+1}+a_{v_{0}+3} t^{v_{0}+3}+\cdots+a_{2 v_{0}-1} t^{2 v_{0}-1}+\sum_{\substack{i>2 v_{0}-1 \\
i \notin \Gamma \Gamma}} a_{i}^{\prime} t^{i},
\end{array}\right.
$$

$\operatorname{com} a_{i}^{\prime}=0$ se $i$ é um dos $s^{\prime}$ primeiros elementos de $\overline{\mathcal{L}}_{s}$.

Dem.: Veja [Z2], théorème 6.1 pag. 131.

Definição 6.1 (Zariski, [Z2] Définition 6.10 pag. 139)

Dizem.os que um ramo admite uma representação paramétrica canônica se este pode ser dado por

$$
\left\{\begin{array}{l}
x=t^{v_{0}} \\
y=t^{v_{0}+1}+a_{v_{0}+3} t^{v_{0}+3}+\cdots+a_{2 v_{0}-1} t^{2 v_{0}-1}+\sum_{i \in \cup_{s=2}^{v_{0}-4} \overline{\mathcal{L}}_{s}} a_{i} t^{i}
\end{array}\right.
$$

com $a_{i}=0$ se $i$ é um dos $s^{\prime}$ primeiros elementos de $\overline{\mathcal{L}}_{s}$ para todo $2 \leq s \leq$ $v_{0}-4$.

Veja que se $s \geq \frac{v_{0}-2}{2}$, então $s^{\prime}=\bar{s}=\sharp\left(\overline{\mathcal{L}}_{s}\right)$. Assim, a parametrização acima definida pode ser reescrita como

$$
\left\{\begin{array}{l}
x=t^{v_{0}} \\
y=t^{v_{0}+1}+a_{v_{0}+3} t^{v_{0}+3}+\cdots+a_{2 v_{0}-1} t^{2 v_{0}-1}+\sum_{i \in \cup_{s=2}^{r} \overline{\mathcal{L}}_{s}} a_{i} t^{i}
\end{array}\right.
$$

onde $r=\left[\frac{v_{0}-3}{2}\right]$ e $a_{i}=0$ se $i$ é um dos $s^{\prime}$ primeiros elementos de $\overline{\mathcal{L}}_{s}$ para todo $2 \leq s \leq r$. 
Observação 6.2 Note que para cada $\Gamma=\left\langle v_{0}, v_{0}+.1\right\rangle$ a parametrização canônica de Zariski não contém representantes de todas as classes de equivalên.cia em $\mathbf{M}_{\Gamma}$. De fato, se $v_{0} \geq 4$ e $\lambda=\left(v_{0}-1\right)\left(v_{0}+1\right)-2 v_{0}, a$ curva

$$
\left\{\begin{array}{l}
x=t^{v_{0}} \\
y=t^{v_{0}+1}+t^{\lambda}
\end{array}\right.
$$

possui invariante de Zariski $\lambda$ e não pode ser expressa sob a forma de uma parametrização canônica de Zariski, uma vez que $\lambda=\left(v_{0}-3\right) v_{0}+\left(v_{0}-3\right)+2 \epsilon$ $\overline{\mathcal{L}}_{v_{0}-3}$ er $r=\left[\frac{v_{0}-3}{2}\right]<v_{0}-3$.

Entretanto, em vista do Teorema 6.1, o ramo genérico admite uma parametrização canônica de Zariski.

Para as parametrizações canônicas genéricas, Zariski apresenta o seguinte. resultado.

Teorema 6.2 Seja $v_{0} \geq 5$ e sejam $C$ e $C^{\prime}$ ramos genéricos que admitem as parametrizações canônicas

$$
C:\left\{\begin{array}{l}
x=t^{v_{0}} \\
y=t^{v_{0}+1}+a_{v_{0}+3} t^{v_{0}+3}+\cdots+a_{2 v_{0}-1} t^{2 v_{0}-1}+\sum_{i \in \cup_{s=2}^{r} \overline{\mathcal{L}}_{s}}^{\dot{i}} a_{i} t^{i}
\end{array}\right.
$$

$e$

$$
C^{\prime}:\left\{\begin{array}{l}
x=t^{v_{0}} \\
y=t^{v_{0}+1}+a_{v_{0}+3}^{\prime} t^{v_{0}+3}+\cdots+a_{2 v_{0}-1}^{\prime} t^{2 v_{0}-1}+\sum_{i \in \cup_{s=2}^{r} \overline{\mathcal{L}}_{s}} a_{i}^{\prime} t^{i} .
\end{array}\right.
$$

Se $C \sim C^{\prime}$, então existe $c \in K^{*}$ tal que $a_{i}=a_{i}^{\prime} c^{i-\left(v_{0}+1\right)}$ para todo $i \geq$ $v_{0}+3$.

Dem.: Veja [Z2] Théorème 6.12 pag. 140.

$\mathrm{Na}$ pag. 141 de [Z2], Zariski observa que o teorema anterior foi verificado para $2 \leq v_{0} \leq 6$ sem a condição de generalidade sobre os parâmetros e levanta a seguinte questão:

"Le Théorème 6.12 (Teorema 6.2)est-il vrai sans hypothèse de généricité sur $C$ et $C^{\prime}$ ?" 
A resposta a esta pergunta é não e um exemplo pode ser dado na classe de equisingularidade determinada por $\Gamma=\langle 7,8\rangle$.

Considere

$$
C:\left\{\begin{array}{l}
x=t^{7} \\
y=t^{8}+t^{10}+t^{11}+\frac{11}{4} t^{12}+a_{13} t^{13}+a_{20} t^{20}
\end{array}\right.
$$

com $a_{13} \neq \frac{21}{4}$.

Obviamente $C$ está sob uma forma paramétrica canônica de Zariski e não é genérica.

Para construir um contraexemplo à pergunta de Zariski, bastará exibir uma mudança de coordenadas como no Exemplo 3.2 que não age como a identidade sobre a parametrização canônica de Zariski.

Note que todo inteiro maior que 27 pertence ao conjunto $\Gamma \cup\left(\Gamma+v_{1}-\right.$ $\left.v_{0}\right) \cup\left(\Gamma^{*}+\lambda\right)$ e portanto, pelo Teorema 4.1 pode ser eliminado de qualquer parametrização sem alterar os termos de ordem inferior.

Desta forma, basta considerar mudanças de coordenadas do tipo

$$
\begin{aligned}
& t_{1}=t\left(1+\frac{p}{t^{7}}\right)^{\frac{1}{7}} \\
& y_{1}=y+q
\end{aligned}
$$

com

$$
p=b_{1} y+b_{2} x^{2}+b_{3} x y+b_{4} y^{2}+b_{5} x^{3}+b_{6} x^{2} * y+b_{7} x y^{2}+b_{8} y^{3}
$$

e

$$
q=c_{1} x^{2}+c_{2} x y+c_{3} y^{2}+c_{4} x^{3}+c_{5} x^{2} y+c_{6} x y^{2}+c_{7} y^{3},
$$

onde não consideramos termos de ordem superior em $p$ e $q$, pois estes só afetariam termos de ordem superior a 27 na parametrização, que são para nós irrelevantes.

Para que uma mụdança de coordenadas como acima mantenha a parametrização canônica de Zariski devemos impor as seguintes condições 
sobre os parâmetros $b$ 's e c's:

$$
\begin{aligned}
& c_{1}=0 \\
& c_{2}=\frac{8}{7} b_{2} \\
& c_{3}=-\frac{12}{7} b_{2} \\
& c_{4}=R_{4}\left(a_{13}, b_{2}\right) \\
& c_{5}=R_{5}\left(a_{13}, b_{2}, b_{5}\right) \\
& c_{6}=R_{6}\left(a_{13}, b_{2}, b_{6}\right) \\
& c_{7}=R_{7}\left(a_{13}, b_{2}, b_{5}, b_{7}\right) \\
& b_{1}=0 \\
& b_{3}=-\frac{3}{2} b_{2} \\
& b_{4}=-\frac{1}{4} b_{2} \\
& b_{5}=S_{5}\left(a_{13}, b_{2}, b_{6}, b_{8}\right) \\
& b_{6}=S_{6}\left(a_{13}, b_{2}, b_{7}, b_{8}\right) \\
& b_{7}=\left(4 a_{13}-21\right)^{-1} S_{7}\left(a_{13}, a_{20}, b_{2}, b_{8}\right),
\end{aligned}
$$

onde $R_{i}$ e $S_{i}$ são certos polinômios nas variáveis indicadas. ${ }^{2}$

- Mas deste modo, teremos

$$
\left\{\begin{array}{l}
x_{1}=t_{1}^{7} \\
y_{1}=t_{1}^{8}+t_{1}^{10}+t_{1}^{11}+\frac{11}{4} t_{1}^{12}+a_{13} t_{1}^{13}+\left(a_{20}+5 b_{2}\left(\frac{3}{4}-\frac{1}{7} a_{13}\right)\right) t_{1}^{20}
\end{array}\right.
$$

Como $a_{13} \neq \frac{21}{4}$, para cada $b_{2} \neq 0$ temos um ramo na parametrização canônica de Zariski equivalente à $C$ que não é obtido por uma homotetia, o que responde negativamente à questão de Zariski.

Vamos utilizar o Algoritmo 2.2 para obter todas as classes de equidiferenciabilidade para $M_{\Gamma} \operatorname{com} \Gamma=\langle 7,8\rangle$ e eliminar alguns parâmetros, obtendo formas normais, que de certo modo, era o que Zariski esperava obter para o caso $v_{0}=7$.

Inicialmente veja que cada classe de equivalência contém um representante da forma

$$
C:\left\{\begin{array}{l}
x=t^{7} \\
y=t^{8}+a_{10} t^{10}+a_{11} t^{11}+a_{12} t^{12}+a_{13} t^{13}+a_{18} t^{18}+ \\
+a_{19} t^{19}+a_{20} t^{20}+a_{26} t^{26}+a_{27} t^{27}+a_{34} t^{34}
\end{array}\right.
$$

Se $a_{i}=0$ para todo $i=10, \ldots, 34$, então $C$ é equivalente à

$$
\left\{\begin{array}{l}
x=t^{7} \\
y=t^{8}
\end{array}\right.
$$

\footnotetext{
${ }^{2}$ Estas contas foram executarlas no MAPLE V Release 5 e os polinômios $R_{i}$ 's c $S_{i}$ 's encontrados estão no anexo ao capítulo.
} 
Vamos supor que existe $a_{i} \neq 0$ para algum

$$
i \in\{10,11,12,13,18,19,20,26,27,34\} \text {, }
$$

i.e., existe invariante $\lambda$ de Zariski.

De acordo com o Corolário 5.1 , se $\lambda \in\{11,12,19,20,26,27,34\}$, então existe apenas uma DNEM $\Omega$ e $v(\Omega)+1=\lambda+7$. Desse modo temos

\begin{tabular}{|c|c|}
\hline$\lambda$ & $\Lambda \backslash \Gamma$ \\
\hline 11 & $18,25,26,33,34,41$ \\
12 & $19,26,27,33,34,41$ \\
19 & $26,33,34,41$ \\
20 & $27,34,41$ \\
26 & 33,41 \\
27 & 34,41 \\
34 & 41 \\
\hline
\end{tabular}

Usando o Teorema 4.1, podemos representar tódos os ramos em cada classe de equidiferenciabilidade dada pelos conjuntos $\Lambda \backslash \Gamma$, listados anteriormente, sob a forma normal

$$
\begin{aligned}
& \left\{\begin{array}{l}
x=t^{7} \\
y=t^{8}+t^{11}+a_{12} t^{12}+a_{13} t^{13}+a_{20} t^{20}
\end{array}\right. \\
& \left\{\begin{array}{l}
\cdot x=t^{7} \\
y=t^{8}+t^{12}+a_{13} t^{13}+a_{18} t^{18}
\end{array}\right. \\
& \left\{\begin{array}{l}
x=t^{7} \\
y=t^{8}+t^{19}+a_{20} t^{20}
\end{array}\right. \\
& \left\{\begin{array}{l}
x=t^{7} \\
y=t^{8}+t^{20}+a_{26} t^{26}
\end{array}\right. \\
& \left\{\begin{array}{l}
x=t^{7} \\
y=t^{8}+t^{26}+a_{27} t^{27}
\end{array}\right. \\
& \left\{\begin{array}{l}
x=t^{7} \\
y=t^{8}+t^{27} \\
y=t^{8}+t^{34}
\end{array}\right.
\end{aligned}
$$


Passemos ao caso $\lambda=10$.

O Teorema 4.1, permite que nos restrinjamos aos ramos da forma

$$
\left\{\begin{array}{l}
x=t^{7} \\
y=t^{8}+t^{10}+a_{11} t^{11}+a_{12} t^{12}+a_{13} t^{13}+a_{19} t^{19}+a_{20} t^{20}+a_{27} t^{27}
\end{array}\right.
$$

Aplicando o Algoritmo 2.2 juntamente com a Proposição 3.3, obtemos as seguintes possibilidades:

\begin{tabular}{|l|l|l|c|}
\hline \multicolumn{2}{|l|}{$a_{12} \neq \frac{13+9 a_{11}^{2}}{8}$} & $\Lambda \backslash \Gamma=\{17,26,27,33,34,41\}$ \\
\hline \multirow{3}{*}{$a_{12}=\frac{13+9 a_{11}^{2}}{8}$} & $a_{13} \neq \frac{78 a_{11}+27 a_{11}^{3}}{20}$ & $\Lambda \backslash \Gamma=\{17,25,27,33,34,41\}$ \\
\cline { 2 - 4 } & $a_{13}=\frac{78 a_{11}+27 a_{11}^{3}}{20}$ & $a_{20} \neq A$ & $\Lambda \backslash \Gamma=\{17,25,33,34,41\}$ \\
\cline { 2 - 4 } & & $a_{20}=A$ & $\Lambda \backslash \Gamma=\{17,25,33,41\}$ \\
\hline
\end{tabular}

onde $A=-\frac{47399}{2560} a_{11}^{2}-\frac{10097}{320} a_{11}^{4}-\frac{17523}{1280} a_{11}^{6}-\frac{357}{512}-\frac{2187}{1280} a_{11}^{8}+\frac{11}{4} a_{11} a_{19}$.

Vejamos como eliminar alguns termos em (6.10) para as três primeiras possibilidades para o conjunto $\Lambda \backslash \Gamma$ dadas acima.

Se $\Lambda \backslash \Gamma=\{17,26,27,33,34,41\}$, então podemos eliminar os termos de ordens 19 e 27, uma vez que estes satisfazem as condições do Lema 4.1. Portanto, podemos nos restringir, neste caso, aos ramos da forma:

$$
\left\{\begin{array}{l}
x=t^{7} \\
y=t^{8}+t^{10}+a_{11} t^{11}+a_{12} t^{12}+a_{13} t^{13}+a_{20} t^{20}
\end{array}\right.
$$

Observe que a parametrização acima coincide com a parametrização canônica de Zariski.

Se $\Lambda \backslash \Gamma=\{17,25,27,33,34,41\}$, então os termos de ordens 20 e 27 também satisfazem as condições do Lema 4.1. Assim, ıessa sitıação, basta considerar os ramos dados por

$$
\left\{\begin{array}{l}
x=t^{7} \\
y=t^{8}+t^{10}+a_{11} t^{11}+\left(\frac{13}{8}+\frac{9}{8} a_{11}^{2}\right) t^{12}+a_{13} t^{13}+a_{19} t^{19}
\end{array}\right.
$$

Se $\Lambda \backslash \Gamma=\{17,25,33,34,41\}$, então podemos eliminar o termo de ordem $l=27$. Note que $l$ não satisfaz as condições do Lema 4.1.

Para eliminar o termo de ordem $l$ sem alterar os de ordem inferior considere $\Omega_{l}=q_{l} d x-p_{l} d y \in \mathcal{O} d \mathcal{O}$ obtida pelo Algoritmo 2.2, tal que $v\left(\Omega_{l}\right)+1=34$. Se considerarmos a mudança de coordenadas

$$
\begin{aligned}
& t_{1}=t\left(1+\frac{\alpha_{1} p_{l}}{t^{7}}\right)^{\frac{1}{7}} \\
& y_{1}=y+q_{l}
\end{aligned}
$$


podemos, para uma escolla conveniente de $\alpha_{l}$, eliminar o termo de ordem $l$, no entanto, estaremos introduzindo termos com ordens inferiores a 27 e superiores a 21 .

Como para cada $j \in\{22,23,24,25,26\}$ existe $\Omega_{j} \in \mathcal{O} d \mathcal{O}$ de modo que $v\left(\frac{\Omega_{j}}{d x}\right)+1=j$, escreva $\Omega_{j}=q_{j} d x-p_{j} d y$ e considere a mundança de coordenadas

$$
\begin{aligned}
& t_{1}=t\left(1+\frac{P}{t^{7}}\right)^{\frac{1}{7}} \\
& y_{1}=y+Q
\end{aligned}
$$

onde $P=\sum_{j=22}^{27} \alpha_{j} p_{j}$ e $Q=\sum_{j=22}^{27} \alpha_{j} q_{j}$.

Ao expandirmos $y_{1}$ em $t_{1}$, veremos que existe uma escolha (única) para $\alpha_{j}$ com $22 \leq j \leq 27$, de modo que podemos eliminar todos os termos com ordem em $\{22, \ldots, 27\}$.

Obtemos assim, a seguinte forma normal

$$
\left\{\begin{aligned}
x=t^{7} & \\
y=t^{8}+t^{10}+\dot{a}_{11} t^{11}+\left(\frac{13}{8}+\frac{9}{8} a_{11}^{2}\right) t^{12}+\left(\frac{39}{10} a_{11}\right. & \left.+\frac{27}{20} a_{11}^{3}\right) t^{13}+ \\
& +a_{19} t^{19}+a_{20} t^{20}
\end{aligned}\right.
$$

Se $\Lambda \backslash \Gamma=\{17,25,33,41\}$, então consideramos (6.10) como forma normal, que devido as restrições impostas, assume a forma

$$
\begin{aligned}
& \left\{\begin{array}{l}
x=t^{7} \\
y=t^{8}+t^{10}+a_{11} t^{11}+\left(\frac{13}{8}+\frac{9}{8} a_{11}^{2}\right) t^{12}+\left(\frac{39}{10} a_{11}+\frac{27}{20} a_{11}^{3}\right) t^{13}+ \\
\quad+a_{19} t^{19}+A t^{20}+a_{27} t^{27},
\end{array}\right. \\
& \operatorname{com} A=-\frac{47399}{2560} a_{11}^{2}-\frac{10097}{320} a_{11}^{4}-\frac{17523}{1280} a_{11}^{6}-\frac{357}{512}-\frac{2187}{1280} a_{11}^{8}+\frac{11}{4} a_{11} a_{19} .
\end{aligned}
$$

Tomemos o caso $\lambda=13$.

Neste caso o Teorema 4.1 permite considerarmos apenas os ramos da forma

$$
\left\{\begin{array}{l}
x=t^{7} \\
y_{c}=t^{8}+t^{13}+a_{18} t^{18}+a_{19} t^{19}+a_{26} t^{26}
\end{array}\right.
$$

A aplicação do Algoritmo 2.2 e da Proposição 3.3 nos fornece as seguintes situaçõès

\begin{tabular}{|c|c|}
\hline restrições & $\Lambda \backslash \Gamma$ \\
\hline$a_{18}=-\frac{1}{2}$ & $20,27,34,41$ \\
\hline$a_{18} \neq-\frac{1}{2}$ & 20,$27 ; 33,34,41$ \\
\hline
\end{tabular}


Se $a_{18} \neq-\frac{1}{2}$, então eliminamos o termo de ordem $l=26$.

Para tanto, consideramos a mundança de coordenadas

$$
\begin{aligned}
& t_{1}=t\left(1-\left(\frac{\alpha_{2} x y+\alpha_{1} y^{3}}{t^{7}}\right)\right)^{\frac{1}{7}} \\
& y_{1}=y+\alpha_{0} y^{3}-\frac{\alpha_{2}}{7}\left(8 y^{2}+5 x^{3}\right) .
\end{aligned}
$$

A expansão de $y_{1}$ em $t_{1}$ mostra que existe uma escolha para $\alpha_{0}, \alpha_{1}$ e $\alpha_{2}$ de forma a eliminar o termo de ordem 26 , sem alterar os de ordem inferior.

Assim, neste caso, basta nos restringirmos ao ramos da forma

$$
\left\{\begin{array}{l}
x=t^{7} \\
y=t^{8}+t^{13}+a_{18} t^{18}+a_{19} t^{19}
\end{array}\right.
$$

Se $a_{18}=-\frac{1}{2}$, então consideramos

$$
\left\{\begin{array}{l}
x=t^{7} \\
y=t^{8}+t^{13}-\frac{1}{2} t^{18}+a_{19} t^{19}+a_{26} t^{26}
\end{array}\right.
$$

como forma normal.

Caso $\lambda=18$.

Para esta situação, o Teorema 4.1 indica que é suficiente considerarmos os ramos da forma

$$
\left\{\begin{array}{l}
x=t^{7} \\
y=t^{8}+t^{18}+a_{19} t^{19}+a_{20} t^{20}+a_{27} t^{27}
\end{array}\right.
$$

Novamente, aplicando o Algoritmo 2.2 e a Proposição 3.3 , obtemos os seguintes casos:

$$
\begin{array}{|c|c|}
\hline \text { restrições } & \Lambda \backslash \Gamma \\
\hline a_{20}=\frac{121}{120} a_{19}^{2} & 25,33,41 \\
\hline a_{20} \neq \frac{21}{120} a_{19}^{2} & 25,33,34,41 \\
\hline
\end{array}
$$

Se $a_{20} \neq \frac{121}{120} a_{19}^{2}$, então podemos eliminar o termo de ordem 27 , sem alterar os de ordem inferior. A mudança de coordenadas utilizada segue os mesmos passos usados para eliminar o termo com esta ordem na situaçãọ $\lambda=10 \mathrm{e}$ $\Lambda \backslash \Gamma=\{17,27,33,34,41\}$.

Assim, temos

$$
\left\{\begin{array}{l}
x=t^{7} \\
y=t^{8}+t^{18}+a_{19} t^{19}+a_{20} t^{20}
\end{array}\right.
$$


Se $a_{20}=\frac{121}{120} a_{19}^{2}$, consideramos a forma normal

$$
\left\{\begin{array}{l}
x=t^{7} \\
y=t^{8}+t^{18}+a_{19} t^{19}+\frac{121}{120} a_{19}^{2} t^{20}+a_{27} t^{27}
\end{array}\right.
$$

Afirmamos que duas formas normais com meșmo $\Lambda \backslash \Gamma$ são equivalentes se, e somente se, diferem por uma homotetia. A verificação deste fato pode ser feita realizando os mesmos procedimentos indicados para o caso do semigrupo $\Gamma=\left\langle 5, v_{1}\right\rangle$ como indicados na pag. 165 .

Apresentamos na sequiência as tabelas com as formas normais e os conjuntos $\Lambda \backslash \Gamma$ para os semigrupos $\langle 6,7\rangle$ e $\langle 7,8\rangle$, onde duas tais formas normais são equivalentes se, e somente se, diferem por uma homotetia.

O caso $\Gamma=\langle 6,7\rangle$, tratado por Zariski em [Z2] no parágrafo 5 do Capítulo $\mathrm{V}$, apesar de não desenvolvido aqui, pode ser obtido com os mesmos métodos usados para o semigrupo $\Gamma=\langle 7,8\rangle$.

\begin{tabular}{|c|c|}
\hline$\Gamma=\langle 6,7\rangle$ & \\
\hline Forma Normal & $\Lambda \backslash \Gamma$ \\
\hline$\left\{\begin{array}{l}x=t^{6} \\
y=t^{7}\end{array}\right.$ & \{\} \\
\hline$\left\{\begin{array}{l}x=t^{6} \\
y=t^{7}+t^{9}+a_{10} t^{10}+a_{11} t^{11} \\
a_{11} \neq \frac{9}{8} a_{10}^{2}+\frac{23}{14}\end{array}\right.$ & $15,22,23,29$ \\
\hline $\begin{array}{l}x=t^{6} \\
y=t^{7}+t^{9}+a_{10} t^{10}+\left(\frac{9}{8} a_{10}^{2}+\frac{23}{14}\right) t^{11}+a_{17} t^{17}\end{array}$ & $15,22,29$ \\
\hline $\begin{array}{l}x=t^{6} \\
y=t^{7}+t^{10}+a_{11} t^{11}\end{array}$ & $16,22,23,29$ \\
\hline $\begin{array}{l}x=t^{6} \\
y=t^{7}+t^{11}+a_{16} t^{16}\end{array}$ & $17,23,29$ \\
\hline $\begin{array}{l}x=t^{6} \\
y=t^{7}+t^{16}+a_{17} t^{17}\end{array}$ & 22,29 \\
\hline$\left\{\begin{array}{l}x=t^{6} \\
y=t^{7}+t^{17}\end{array}\right.$ & 23,29 \\
\hline $\begin{array}{l}x=t^{6} \\
y=t^{7}+t^{23}\end{array}$ & 29 \\
\hline
\end{tabular}




\begin{tabular}{|c|c|}
\hline$\Gamma=\langle 7,8\rangle$ & \\
\hline Forma Normal & $\Lambda \backslash \Gamma$ \\
\hline$\left\{\begin{array}{l}x=t^{7} \\
y=t^{8}\end{array}\right.$ & \{\} \\
\hline $\begin{array}{l}x=t^{7} \\
y=t^{8}+t^{10}+a_{11} t^{11}+a_{12} t^{12}+a_{13} t^{13}+a_{20} t^{20} \\
a_{12} \neq \frac{13}{8}+\frac{9}{8} a_{11}^{2}\end{array}$ & $17,25,26,33,34,41$ \\
\hline$\left\{\begin{array}{l}x=t^{7} \\
y=t^{8}+t^{10}+a_{11} t^{11}+a_{12} t^{12}+a_{13} t^{13}+a_{19} t^{19} \\
\quad a_{12}=\frac{13}{8}+\frac{9}{8} a_{11}^{2} \text { e } a_{13} \neq \frac{39}{11} a_{11}+\frac{27}{20} a_{11}^{3}\end{array}\right.$ & $17,25,27,33,34,41$ \\
\hline $\begin{array}{l}\left\{\begin{array}{l}x=t^{7} \\
y=t^{8}+t^{10}+a_{11} t^{11}+a_{12} t^{12}+ \\
\\
\quad+a_{13} t^{13}+a_{19} t^{19}+a_{20} t^{20}\end{array}\right. \\
a_{12}=\frac{13}{8}+\frac{9}{8} a_{11}^{2}, a_{13}=\frac{39}{11} a_{11}+\frac{27}{20} a_{11}^{3} \text { e } a_{20} \neq A\end{array}$ & $17,25,33,34,41$ \\
\hline $\begin{array}{l}\left\{\begin{array}{l}x=t^{7} \\
y=t^{8}+t^{10}+a_{11} t^{11}+a_{12} t^{12}+ \\
\\
\quad+a_{13} t^{13}+a_{19} t^{19}+a_{20} t^{20}+a_{27} t^{27}\end{array}\right. \\
a_{12}=\frac{13}{8}+\frac{9}{8} a_{11}^{2}, a_{13}=\frac{39}{11} a_{11}+\frac{27}{20} a_{11}^{3} \text { e } a_{20}=A\end{array}$ & $17,25,34,41$ \\
\hline$\left\{\begin{array}{l}x=t^{7} \\
y=t^{8}+t^{11}+a_{12} t^{12}+a_{13} t^{13}+a_{20} t^{20}\end{array}\right.$ & $18,25,26,33,34,41$ \\
\hline$\left\{\begin{array}{l}x=t^{7} \\
y=t^{8}+t^{12}+a_{13} t^{13}+a_{18} t^{18}\end{array}\right.$ & $19,26,27,33,34,41$ \\
\hline$\left\{\begin{array}{l}x=t^{7} \\
y=t^{8}+t^{13}+a_{18} t^{18}+a_{19} t^{19} \\
a_{18} \neq-\frac{1}{2}\end{array}\right.$ & $20,27,33,34,41$ \\
\hline $\begin{array}{l}x=t^{7} \\
y=t^{8}+t^{13}-\frac{1}{2} t^{18}+a_{19} t^{19}+a_{26} t^{26}\end{array}$ & $20,27,34,41$ \\
\hline$\left\{\begin{array}{l}x=t^{7} \\
y=t^{8}+t^{18}+a_{19} t^{19}+a_{20} t^{20} \\
\quad a_{20} \neq \frac{121}{120} a_{19}^{2}\end{array}\right.$ & $25,33,34,41$ \\
\hline $\begin{array}{l}x=t^{7} \\
y=t^{8}+t^{18}+a_{19} t^{19}+\frac{121}{120} a_{19}^{2} t^{20}+a_{27} t^{27}\end{array}$ & $25,33,41$ \\
\hline
\end{tabular}




\begin{tabular}{|c|c|}
\hline \multicolumn{1}{|c|}{ Forma Normal } & $\Lambda \backslash \Gamma$ \\
\hline$\left\{\begin{array}{l}x=t^{7} \\
y=t^{8}+t^{19}+a_{20} t^{20}\end{array}\right.$ & $26,33,34,41$ \\
$\left\{\begin{array}{l}x=t^{7} \\
y=t^{8}+t^{20}+a_{26} t^{26}\end{array}\right.$ & $27,34,41$ \\
\hline$\left\{\begin{array}{l}x=t^{7} \\
y=t^{8}+t^{26}+a_{27} t^{27}\end{array}\right.$ & 33,41 \\
\hline$\left\{\begin{array}{l}x=t^{7} \\
y=t^{8}+t^{27}\end{array}\right.$ & 34,41 \\
\hline$\left\{\begin{array}{l}x=t^{7} \\
y=t^{8}+t^{34}\end{array}\right.$ \\
\hline
\end{tabular}

\section{ANEXO}

Neste anexo apresentamos explicitamente os polinômios $R_{i}$ 's e $S_{i}$ 's mencionados na pag. 173 .

$$
\begin{aligned}
& R_{4}\left(a_{13} ; b_{2}\right)=-\frac{135}{28} b_{2}-\frac{15}{14} a_{13} b_{2} \\
& R_{5}\left(a_{13}, b_{2}, b_{5}\right)=-\frac{25}{4} b_{2}-\frac{29}{28} a_{13} b_{2}+\frac{8}{7} b_{5}+\frac{4}{49} b_{2}^{2} \\
& R_{6}\left(a_{13}, b_{2}, b_{6}\right)=-\frac{3}{2} a_{13} b_{2}+\frac{8}{7} b_{6}-\frac{641}{56} b_{2}-\frac{12}{49} b_{2}^{2} \\
& R_{7}\left(a_{13}, b_{2}, b_{5}, b_{7}\right)=-\frac{52}{7} b_{2}+\frac{8}{7} b_{7}-\frac{81}{28} a_{13} b_{2}+\frac{2}{7} b_{5}+\frac{18}{49} b_{2}^{2} \\
& \cdots S_{5}\left(a_{13}, b_{2}, b_{6}, b_{8}\right)=\frac{4}{7} b_{2}^{2}-\frac{227}{6} b_{2}+\frac{199}{24} a_{13} b_{2}-\frac{2}{3} b_{6}-\frac{8}{3} b_{8} \\
& S_{6}\left(a_{13}, b_{2}, b_{7}, b_{8}\right)=6 b_{7}-\frac{45}{2} b_{2} a_{13}^{2}+\frac{9565}{16} b_{2}-40 b_{8}+\frac{72}{7} b_{2}^{2}+\frac{4297}{16} a_{13} b_{2} \\
& S_{7}\left(a_{13}, a_{20}, b_{2} ; b_{8}\right)=\frac{1}{1120}\left(-2720 a_{13} b_{2}^{2}+557690 a_{13} b_{2}-277200 b_{2} a_{13}^{2}+\right. \\
& +16800 b_{2} a_{13}^{3}+26880 a_{13} b_{8}+2459289 b_{2}-141120 b_{8}+ \\
& +14280 b_{2}^{2}+2688 b_{2} a_{20} \text { ) }
\end{aligned}
$$




\section{Apêndice A}

\section{Semigrupos do tipo $\left\langle 2 n_{1}, 2 m_{1}, v_{2}\right\rangle$}

Em [LuP], Luengo e Pfister mostram que o número $\tau$ de Tjurina é constante para todos os ramos cujo semigrupo de valores é $\Gamma=\left\langle v_{0}, v_{1}, v_{2}\right\rangle$ fixado, com $e_{1}=\mathrm{MDC}\left(v_{0}, v_{1}\right)=2$. A saber, $\tau=\mu-\left(n_{1}-1\right)\left(m_{1}-1\right)$, onde $n_{1}=\frac{v_{0}}{e_{1}}$ e $m_{1}=\frac{v_{1}}{e_{1}}$. Conseqüentemente, temos a constância do número de lacunas especiais, ou seja, $\sharp(\Lambda \backslash \Gamma)=\left(n_{1}-1\right)\left(m_{1}-1\right)$. Isto não quer dizer que o conjunto $\Lambda \backslash \Gamma$ seja sempre o mesmo, como teremos oportunidade de verificar.

Nesta seção, mostraremos como o algoritmo para obter uma Base Standard Mínima de $\mathcal{O} d \mathcal{O}$ pode ser otimizado, no sentido de que poderemos indicar precisamente o $S$-processo cuja redução final origina uma DNEM para semigrupos da forma mencionanda. Além disso, indicaremos como os diagramas de lacunas especiais, ou equivalentemente, os conjuntos de lacunas especiais, que ocorrem para curvas que admitem tais semigrupos estão totalmente determinados pelas lacunas especiais do primeiro bloco, on seja, pelas lacunas da forma $v(\Omega)+1 \operatorname{com} \Omega \in \mathcal{O} d \mathcal{O} \mathrm{DNE}$ 'do primeiro bloco.

Todo ramo com semigrupo $\Gamma=\left\langle v_{0}, v_{1}, v_{2}\right\rangle$ pode ser representado por

$$
C:\left\{\begin{array}{l}
x=t^{v_{0}} \\
y=t^{v_{1}}+\sum_{v_{1}<i<\beta_{2}} a_{i} t^{i}+a_{\beta_{2}} t^{\beta_{2}}+\sum_{i>\beta_{2}} a_{i} t^{i},
\end{array}\right.
$$

onde $\beta_{0}=v_{0}, \beta_{1}=v_{1}$ e $\beta_{2}$ é a seqüểncia característica, $a_{i} \in K, a_{\beta_{2}} \in K^{*} \mathrm{e}$ $e_{1} \mid i$ para todo $i<\beta_{2}$ com $a_{i} \neq 0$.

Uma vez que $v_{2}=\left(n_{1}-1\right) v_{1}+\beta_{2}$ e $n_{1} v_{1}=m_{1} v_{0}$, podemos escrever $\beta_{2}=v_{2}+v_{1}-m_{1} v_{0}$.

O diagrama de lacunas especiais, que estará contido em $\mathbb{N}^{3}$, possui apenas dois blocos, o primeiro bloco composto pelos elementos de $\Lambda \backslash \Gamma$ que são divisíveis por $e_{1}$, que são da forma $s_{1} v_{1}-s_{0} v_{0}$ com $1<s_{1}<n_{1}$ e $0<s_{0}$, e o 
segundo, formado pelos outros elementos, que são da forma $v_{2}+s_{1} v_{1}-s_{0} v_{0}$ com $0 \leq s_{1}<n_{1}$ e $s_{0}>0$, já que $n_{2}=2$.

Sabemos que o invariante $\lambda$ de Zariski é tal que $v_{1}<\lambda \leq \beta_{2}$ (Veja parágrafo após Observação 3.2).

Vejamos inicialmente o caso extremo, isto é, quando $\lambda=\beta_{2}$.

Nesse caso, $h_{2}=y^{n_{1}}-x^{m_{1}}$ é tal que $v\left(h_{2}\right)=v_{2}$ e $F=\left\{x, y, h_{2}\right\}$ é uma Base Standard Mínima para $\mathcal{O}$ (Veja Proposição 2.1).

Iniciando com $B=\left\{d x, d y, d h_{2}\right\}$ e aplicando o algoritmo para obter uma Base Standard Mínima para $\mathcal{O} d \mathcal{O}$, temos que $\Omega_{1}=x d y-\frac{m_{1}}{n_{1}} y d x$ é uma DNEM com $v\left(\Omega_{1}\right)+1=\lambda+v_{0}=v_{2}+v_{1}-\left(m_{1}-1\right) v_{0}$. Analisando os demais $S$-processos mínimos vemos que, se existisse uma outra DNEM $\Omega$, então $v(\Omega)+1>v_{2}$, ou seja, $\Omega$ pertenceria ao segundo bloco. Portanto, deveríamos ter

$$
v(\Omega)+1=v_{2}+\alpha v_{1}-\gamma v_{0},
$$

com $0<\alpha<n_{1}$ e $0<\gamma<m_{1}$.

Mas, dessa forma

$$
\begin{aligned}
v_{2}+\alpha v_{1}-\gamma v_{0} & =v_{2}+v_{1}-\left(m_{1}-1\right) v_{0}+(\alpha-1) v_{1}+\left(m_{1}-1-\gamma\right) v_{0}= \\
& =v\left(\Omega_{1}\right)+1+(\alpha-1) v_{1}+\left(m_{1}-1-\gamma\right) v_{0},
\end{aligned}
$$

com $(\alpha-1) v_{1}+\left(m_{1}-1-\gamma\right) v_{0} \in \Gamma$. Contrariando o fato de $\Omega$ ser DNEM.

Portanto, o algoritmo finaliza com apenas uma DNEM $\Omega_{1}$ e temos

$$
\Lambda \backslash \Gamma=\left\{v_{1}+\alpha v_{1}-\gamma v_{0} ; 0<\alpha<n_{1} \text { e } 0<\gamma<m_{1}\right\}
$$

oil seja, $\sharp(\Lambda \backslash \Gamma)=\left(n_{1}-1\right)\left(m_{1}-1\right)$.

Para o caso em que $\lambda<\beta_{2}$, apresentaremos determinados $S$-processos e mostraremos que suas reduções finais correspondem a DNE, em seguida procedemos a contagem das DNE da forma $F^{\delta} \Omega$, onde $\delta \in \mathbb{N}^{3}$ e $F=\left\{x, y, h_{2}\right\}$ é uma Base Standard Mínima para $\mathcal{O}$, dada como na Proposição 2.1 e $\Omega$ uma DNE construída. Constataremos que existem $\left(n_{1}-1\right)\left(m_{1}-1\right)$ tais DNE, conseqüentemente, pelo resultado de Luengo e Pfister, estas DNE são na verdade todas.

Relembremos as notações do Capítulo 3, mais especificamente as envolvidas na Proposição 3.3. Denotando $\Omega_{-1}=d x$ e $\Omega_{0}=d y$, temos que se $\left\{\Omega_{1}, \ldots, \Omega_{i}\right\}$ com $v\left(\Omega_{k}\right)<v\left(\Omega_{k+1}\right)$ para $k=1, \ldots, i-1$ são as DNEM's do primeiro bloco, obtidas pela aplicação do algoritmo da Proposição 3.3 até $11 \mathrm{~m}$ determinado passo, então havendo uma outra $\mathrm{DNEM} \Omega_{i+1}$ no. primeiro bloco 
$\operatorname{com} v\left(\Omega_{i}\right)<v\left(\Omega_{i+1}\right)$, esta pode ser obtida pela redução final do $S$-processo $S\left(\Omega_{i}, \Omega_{j}\right)$, com $h t\left(S\left(\Omega_{i}, \Omega_{j}\right)\right)=h t_{i}$, onde

$$
h t_{i}=\min \left\{h t_{x}, h t_{y}\right\}, \quad h t^{i}=\max \left\{h t_{x}, h t_{y}\right\}
$$

$h t_{x}=\min _{-1 \leq j<i}\left\{\right.$ menor altura de um $S$ - processo da forma $\left.x^{\alpha} \Omega_{i}-a y^{\gamma} \Omega_{j}\right\}$, denominado de altura mínima dos $S$-processos de $\Omega_{i}$ na direção $x$, e

$h t_{y}=\min _{-1 \leq j<i}\left\{\right.$ menor altura de um $S-$ processo da forma $\left.y^{\alpha} \Omega_{i}-a x^{\gamma} \Omega_{j}\right\}$, denominado de altura mínima dos $S$-processos de $\Omega_{i}$ na direção $y$.

Desse modo, para as DNEM's do primeiro bloco, os $S$-processos essenciais, são como os indicados na Proposição 3.3.

Vamos mostrar que para os semigrupos que estamos considerando, i.e., aqueles da forma $\Gamma=\left\langle 2 n_{1}, 2 m_{1}, v_{2}\right\rangle$, as DNEM's do segundo bloco são obtidas por uma redução fiulal, módulo as DNEM's do primeiro bloco, dos $S$-processos da forma $S\left(\Omega_{i}, \Omega_{j}\right)$, com $h t\left(S\left(\Omega_{i}, \Omega_{j}\right)\right)=h t^{i}$ e $-1 \leq j<i$, para cada DNEM $\Omega_{i}$ do primeiro bloco.

Antes porém, necèssitamos de alguus resultados auxiliares.

Definição A.1 Se $\Omega \in \mathcal{O} d \mathcal{O}$, definimos $\ln (\Omega)$ como sendo a menor ordem $s$ de um termo de $\psi(\Omega)$, tal que $e_{1} \chi(s+1)$, onde $\psi$ é o homomorfismo definido em (2.2). Se tal termo não existe, definimos $\ln (\Omega)=\infty$.

Lema A.1 Sejam $\left\{\Omega_{1}, \ldots, \Omega_{r}\right\}$ o conjunto das DNEM's do primeiro bloco, $\Omega_{-1}=d x$ e $\Omega_{0}=d y$. Escreva $\Omega_{i}=q_{i} d x-p_{i} d y$ e defina $v\left(G^{i}\right)=v\left(h t_{i}\right)-v\left(\Omega_{i}\right)$ para $i=0, \ldots, r-1$. Então $\ln \left(\Omega_{0}\right)=\beta_{2}-1$ e para $i=0, \ldots, r-1$,

$$
\ln \left(\Omega_{i+1}\right)=v\left(G^{i}\right)+v\left(p_{i}\right)+\beta_{2}-1=v\left(p_{i+1}\right)+\beta_{2}-1,
$$

e para todo $\alpha, \delta \in \mathbb{N}$,

$$
\ln \left(x^{\alpha} y^{\delta} \Omega_{i+1}\right)=\alpha v_{0}+\delta v_{1}+\dot{l n}\left(\Omega_{i+1}\right) .
$$

Dem.: Consideremos os ramos da forma

$$
\left\{\begin{array}{l}
x=t^{v_{0}} \\
y=t^{v_{1}}+\sum_{v_{1}<i<\beta_{2}} a_{i} t^{i}+a_{\beta_{2}} t^{\beta_{2}}+\sum_{i>\beta_{2}} a_{i} t^{i},
\end{array}\right.
$$

com semigrupo de valores $\Gamma=\left\langle v_{0}, v_{1}, v_{2}\right\rangle$. 
Observe inicialmente que na expansão em $t$ de um monồmio da forma $x^{\alpha} y^{\delta}$, o termo de menor ordem cujo expoente nã̃o é divisivel por $e_{1}$ é $\delta a_{\beta_{2}} t^{\alpha v_{0}+(\delta-1) v_{1}+\beta_{2}}=\delta a_{\beta_{2}} t^{v\left(x^{\alpha} y^{\delta}\right)+\beta_{2}-v_{1}}$.

Desse modo, é fácil verificar que

$$
\ln \left(x^{\alpha} y^{\delta} d x\right)=\alpha v_{0}+(\delta-1) v_{1}+\beta_{2}+v_{0}-1=v\left(x^{\alpha} y^{\delta} d x\right)+\beta_{2}-v_{1}
$$

cujo coeficiente do termo correspondente é $v_{0} \delta a_{\beta_{2}}$ e

$$
\ln \left(x^{\alpha} y^{\delta} d y\right)=\alpha v_{0}+(\delta-1) v_{1}+\beta_{2}+v_{1}-1=v\left(x^{\alpha} y^{\delta} d y\right)+\beta_{2}-v_{1}
$$

e o coeficiente do termo correspondente é $\left(\delta v_{1}+\beta_{2}\right) a_{\beta_{2}}$.

Conseqüentemente, se $v\left(x^{\alpha_{1}} y^{\delta_{1}} \Omega_{i}\right)<v\left(x^{\alpha_{2}} y^{\delta_{2}} \Omega_{j}\right)$, com $\Omega_{i}, \Omega_{j} \in\{d x, d y\}$, então

$$
\ln \left(x^{\alpha_{1}} y^{\delta_{1}} \Omega_{i}+x^{\alpha_{2}} y^{\delta_{2}} \Omega_{j}\right)=\ln \left(x^{\alpha_{1}} y^{\delta_{1}} \Omega_{i}\right)
$$

Uma vez que

$$
\begin{aligned}
& \Omega_{1}=\left(-\frac{m_{1}}{n_{1}} y+\cdots\right) d x-(-x+\cdots) d y= \\
& .=x d y-\frac{m_{1}}{n_{1}} y d x+\sum_{\alpha=\left(\boldsymbol{\alpha}_{1}, \alpha_{2}\right) \in \mathbb{N}^{2}} b_{\alpha} x^{\alpha_{1}} y^{\boldsymbol{\alpha}_{2}} \Omega_{\alpha},
\end{aligned}
$$

$\operatorname{com} \Omega_{\alpha} \in\{d x, d y\}, v\left(x^{\alpha_{1}} y^{\alpha_{2}} \Omega_{\alpha}\right)>v(x d y)=v(y d x)$ e $x d y-\frac{m_{1}}{n_{1}} y d x=$ $\sum_{i \geq \lambda} a_{i}\left(i-v_{1}\right) t^{i+v_{0}-1} d t$, temos que

$$
\begin{aligned}
\ln \left(x d y-\frac{m_{1}}{n_{1}} y d x\right) & =\beta_{2}+v_{0}-1=v_{1}+v_{0}-1+\beta_{2}-v_{1}= \\
& =\ln (x d y)=\ln (y d x)<\ln \left(x^{\alpha_{1}} y^{\alpha_{2}} \Omega_{\alpha}\right) .
\end{aligned}
$$

Segue assim que

$$
\ln \left(\Omega_{1}\right)=\ln \left(x d y-\frac{m_{1}}{n_{1}} y d x\right)=\ln (x d y)=v_{0}+\beta_{2}-1=v\left(G^{0}\right)+v\left(p_{0}\right)+\beta_{2}-1,
$$

e portanto pelo Lema 3.2

$$
\ln \left(\Omega_{1}\right)=v\left(p_{1}\right)+\beta_{2}-1 .
$$

Como o coeficiente de $t^{\ln \left(x^{\alpha} y^{\delta} y d x\right)}$ em $x^{\alpha} y^{\delta} y d x$ é $v_{0}(\delta+1) a_{\beta_{2}}$ e o de $t^{l n\left(x^{\alpha} y^{\delta} x d y\right)}$ em $x^{\alpha} y^{\delta} x d y$ é $\left(\beta_{2}+\delta v_{1}\right) a_{\beta_{2}}$, o termo em $x^{\alpha} y^{\delta}\left(x d y-\frac{m_{1}}{n_{1}} y d x\right)$ de menor ordem, cujo expoente, acrescido de 1 , não é divisível por $e_{1}$ tem coeficiente $\left(\beta_{2}+\delta v_{1}-\frac{m_{1}}{n_{1}} v_{0}(\delta+1)\right) a_{\beta_{2}}=\left(\beta_{2}-v_{1}\right) a_{\beta_{2}} \neq 0$.

Desse modo, o mesmo argumento usado para $\Omega_{1}$, nos leva a concluir que

$$
\ln \left(x^{\alpha} y^{\delta} \Omega_{1}\right)=\ln \left(x^{\alpha} y^{\delta} x d y\right)=\alpha v_{0}+\delta v_{1}+\ln \left(\Omega_{1}\right),
$$


o que prova o lema para $i=0$.

Suponha que o lema seja verdadeiro para todo $j$ com $0 \leq j \leq i<r-1$ e considere

$$
\Omega_{i+1}=F^{\alpha} \Omega_{i}+F^{\delta} \Omega_{k}+\sum_{l=-1}^{i} F^{\alpha_{l}} \Omega_{l},
$$

como na Proposição 3.3, onde $v\left(F^{\alpha} \Omega_{i}\right)=v\left(F^{\delta} \Omega_{k}\right)=v\left(h t_{i}\right)$ para $-1 \leq k<i$ e $F=\{x, y\}$.

Uma vez que

$$
\ln \left(F^{\alpha_{-1}} \Omega_{-1}\right)=v\left(F^{\alpha_{-1}}\right)+\ln \left(\Omega_{-1}\right)=v\left(F^{\alpha_{-1}}\right)+v_{0}-v_{1}+\beta_{2}-1,
$$

e, por hipótese de indução, que

$$
\ln \left(F^{\alpha_{j}} \Omega_{j}\right)=v\left(F^{\alpha_{j}}\right)+\ln \left(\Omega_{j}\right)=v\left(F^{\alpha_{j}}\right)+v\left(p_{j}\right)+\beta_{2}-1,
$$

para todo $j$ tal que $0 \leq j \leq i$, então segue da Observação 3.7 que

$$
\ln \left(\Omega_{i+1}\right)=\ln \left(F^{\alpha_{i}} \Omega_{i}\right)=v\left(F^{\alpha_{i}}\right)+v\left(p_{i}\right)+\beta_{2}-1 .
$$

Como $v\left(h t_{i}\right)=v\left(F^{\alpha_{i}}\right)+v\left(\Omega_{i}\right)$, temos que $v\left(F^{\alpha_{i}}\right)=v\left(G^{i}\right)$, e assim pelo Lema 3.2

$$
\begin{aligned}
\ln \left(\Omega_{i+1}\right) & =v\left(G^{i}\right)+v\left(p_{i}\right)+\beta_{2}-1 \\
& =v\left(p_{i+1}\right)+\beta_{2}-1
\end{aligned}
$$

provando parte do resultado.

Agora, como $v\left(\Omega_{i+1}\right)>v\left(p_{i+1} d y\right)=v\left(p_{i+1}\right)+v_{1}-1$, temos que $\ln \left(x^{\alpha} y^{\delta} \Omega_{i+1}\right)$, assume o valor

$$
\begin{aligned}
\alpha v_{0}+ & \min \left\{\delta v_{1}+v\left(p_{i+1}\right)+\beta_{2}-1,(\delta-1) v_{1}+\beta_{2}+v\left(\Omega_{i+1}\right)\right\}= \\
= & \alpha v_{0}+\delta v_{1}+v\left(p_{i+1}\right)+\beta_{2}-1=\alpha v_{0}+\delta v_{1}+\ln \left(\Omega_{i+1}\right),
\end{aligned}
$$

e conseqüentemente,

$$
\ln \left(x^{\alpha} y^{\delta} \Omega_{i+1}\right)=\alpha v_{0}+\delta v_{1}+\ln \left(\Omega_{i+1}\right) .
$$

No Capítulo 3, vimos que todo elemento de $\mathcal{O} d \mathcal{O}$ cuja ordem șeja maior ou igual a $v\left(h t^{i}\right)$, para algum $i=-1, \ldots, r$, terá redução final, módulo $\left\{\Omega_{-1}, \ldots, \Omega_{i}\right\}$, igual a zero ou pertencerá a blocos superiores. 
Usando o lema anterior, podemos avaliar a ordem das DNE's obtidas por uma redução final, módulo as DNEM's do primeiro bloco, de. um $S$-processo $S\left(\Omega_{i}, \Omega_{j}\right)=F^{\alpha_{i}} \Omega_{i}-F^{\alpha_{j}} \Omega_{j}$ com altura $h t^{i}$, onde $F=\{x, y\}$. De fato, como mencionamos acima, enquanto tivermos termos cujas ordens mais 1 sejam divisíveis por $e_{1}$, podemos prossegir com o processo de redução.

Resta assim avaliar qual a menor ordem de um termo que ocorrerá no processo de redução, tal que acrescido de uma unidade não seja divisível por $e_{1}$.

Seja

$$
\Omega=F^{\alpha_{i}} \Omega_{i}-F^{\alpha_{j}} \Omega_{j}+\sum_{k=-1}^{i} F^{\delta_{k}} \Omega_{k},
$$

$\operatorname{com} v\left(h t^{i}\right)=v\left(F^{\alpha_{i}} \Omega_{i}\right)=v\left(F^{\alpha_{j}} \Omega_{j}\right)<v\left(F^{\delta_{k}} \Omega_{k}\right)$, para todo $k=-1, \ldots, i$.

Pcla Observação 3.7, temos que

$$
v\left(F^{\alpha_{i}}\right)+v\left(p_{i}\right)<\min _{-1 \leq k<i}\left\{v\left(F^{\alpha_{k}}\right)+v\left(p_{k}\right)\right\} .
$$

Desse modo, segue do lema anterior que podemos reduzir o $S$-processo $F^{\alpha_{i}} \Omega_{i}-F^{\alpha_{j}} \Omega_{j}$, módulo $\left\{\Omega_{-1}, \ldots, \Omega_{i}\right\}$, de modo a obtermos $\Omega^{i}$ no segundo bloco, com

$$
v(\Omega)+1=\ln \left(F^{\alpha_{i}} \Omega_{i}\right)+1=v\left(F^{\alpha_{i}}\right)+v\left(p_{i}\right)+\beta_{2},
$$

que obviamente não é divisível por $e_{1}$, pois $v\left(F^{\alpha_{i}}\right)$ e $v\left(p_{i}\right)$ o são e $\beta_{2}$ não.

Observação A.1 Denotaremos por $G^{i}$ e por $F^{i}$ os $\{x, y\}$-produtos de potências, tais que

$$
v\left(G^{i}\right)=v\left(h t_{i}\right)-v\left(\Omega_{i}\right)
$$

$e$

$$
v\left(F^{i}\right)=v\left(h t^{i}\right)-v\left(\Omega_{i}\right) .
$$

Note que pelo lema anterior $\ln \left(\Omega_{i+1}\right)=v\left(G^{i}\right)+v\left(p_{i}\right)+\beta_{2}-1$. Como $v\left(\Omega^{i}\right)=\ln \left(F^{i} \Omega_{i}\right)=v\left(F^{i}\right)+v\left(p_{i}\right)+\beta_{2}-1$, temos que

$$
\ln \left(\Omega_{i+1}\right)=v\left(\Omega^{i}\right)-v\left(F^{i}\right)+v\left(G^{i}\right) .
$$

O próximo passo será avaliar explicitamente $v\left(\Omega^{i}\right)+1$. 
Proposição A.1 Sejam $\left\{\Omega_{-1}, \ldots, \Omega_{r}\right\}$ o conjunto das DNEM's do primeiro bloco, $\Omega_{-1}=d x$ e $\Omega_{0}=d y$. Se $\left\{v\left(\Omega_{j}\right)+1 ; j=1, \ldots, r\right\}=\left\{\alpha_{i} v_{1}-\delta_{i} v_{0} ; i=\right.$ $1, \ldots, r\}$, onde a representação usada para $\alpha_{i} v_{1}-\delta_{i} v_{0}$ é a escrita privilegiada (Veja pag. 69). Então as ordens das DNE's do segundo bloco, obtidas por uma redução final módulo $\left\{\Omega_{-1}, \ldots, \Omega_{r}\right\}$, de $S$-processos $S\left(\Omega_{i}, \Omega_{j}\right)$ com altura $h t_{i}$ ou $h t^{i}$ são da forma

$$
v_{2}+\left(n_{1}-\alpha_{i+1}+1\right) v_{1}-\left(m_{1}-\delta_{i}-1\right) v_{0}
$$

para $i=0, \ldots, r$, onde $\delta_{0}=0, \alpha_{0}=1, \delta_{r+1}=m_{1}-1$ e $\alpha_{r+1}=n_{1}$.

Dem.: Indicaremos as diferenciais do primeiro bloco por $\Omega_{k}$ e as do segundo por $\Omega^{k}$.

Suponha $\Omega_{1}$ tal que $v\left(\Omega_{1}\right)+1=\alpha v_{1}-\delta v_{0}$. Assim, $\alpha_{0}<\alpha<\alpha_{r+1}$ e $\delta_{0}<\delta<\delta_{r+1}$.

Como na observação anterior, indicamos por $G^{1} \Omega_{1}$ e por $F^{1} \Omega_{1}$ os elementos de menor e de maior ordem do conjunto $\left\{y^{\alpha_{r+1}-\alpha} \Omega_{1}, x^{\delta-\delta_{0}} \Omega_{1}\right\}$.

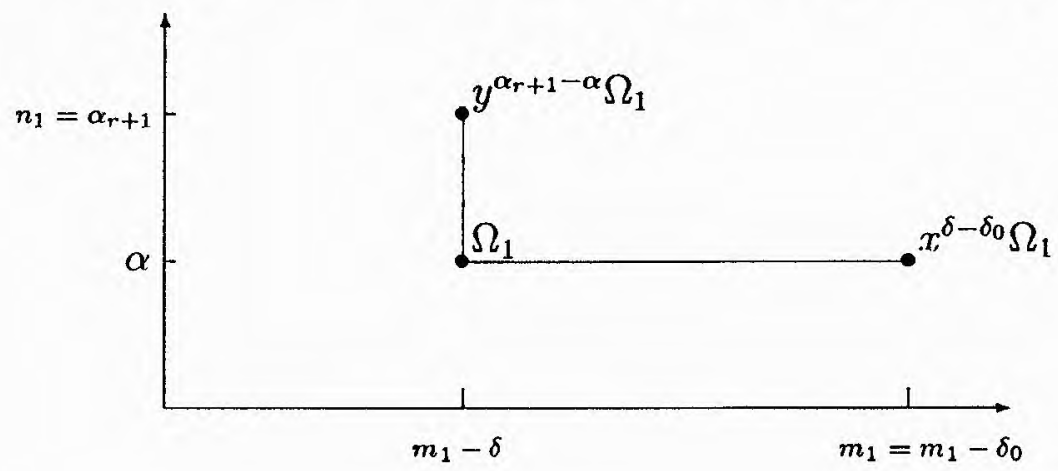

Duas possibilidades podem ocorrer:

Caso a) Não existe uma outra DNEM do primeiro bloco.

Nesse caso, $r=1, \alpha_{1}=\alpha, \delta_{1}=\delta$ e temos 2 DNE's no segundo bloco, obtidas por uma redução final, módulo $\left\{d x, d y, \Omega_{1}\right\}$, dos $S$-processos envolvèndo $G^{1} \Omega_{1}$ e $F^{1} \Omega_{1}$, que pelo lema anterior possuem ordens:

$$
\begin{aligned}
\left(\alpha_{r+1}-\alpha\right) v_{1}+\ln \left(\Omega_{1}\right) & =\left(\alpha_{2}-\alpha_{1}\right) v_{1}+v_{0}+\beta_{2}-1= \\
& =v_{2}+\left(n_{1}-\alpha_{1}+1\right) v_{1}-\left(m_{1}-1\right) v_{0}-1,
\end{aligned}
$$

e 


$$
\begin{aligned}
\left(\delta-\delta_{0}\right) v_{0}+\ln \left(\Omega_{1}\right) & =\left(\delta_{1}-\delta_{0}\right) v_{0}+v_{0}+\beta_{2}-1= \\
& =v_{2}+v_{1}-\left(m_{1}-\delta_{1}-1\right) v_{0}-\dot{1}
\end{aligned}
$$

provando o resultado para $i=1$ neste caso.

Caso b) A redução fiual do $S$-processo envolvendo $G^{1} \Omega_{1}$, módulo $\left\{d x, d y, \Omega_{1}\right\}$, origina uma nova DNEM $\Omega_{2}$ no primeiro bloco. Nesse caso, dois fenômenos podem ocorrer:

$\therefore G^{1}=y^{\alpha_{r+1}-\alpha} \Omega_{1}$.

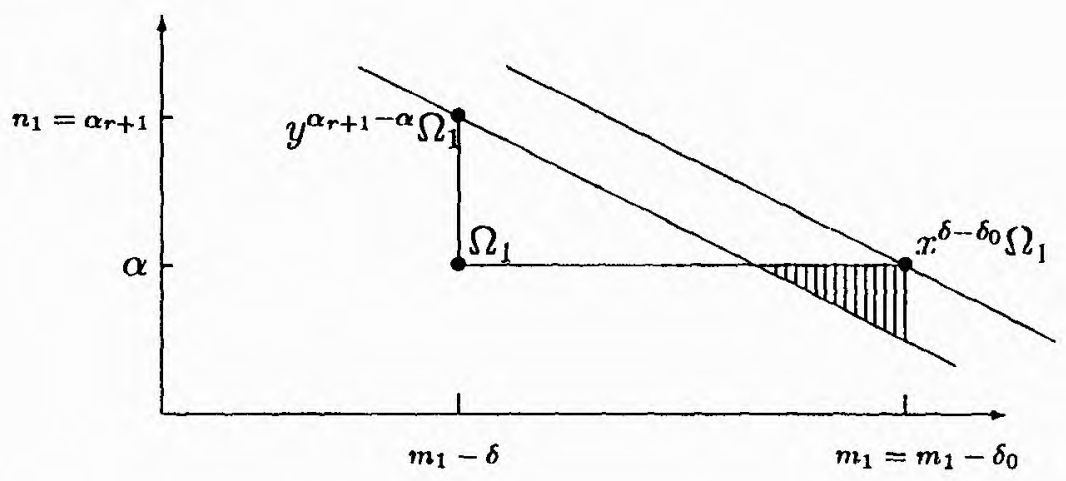

Isto implica que $\alpha_{r}=\alpha, \delta_{r}=\delta$ e que a redução final do $S$-processo envolvendo $F^{1} \Omega_{1}$ nos dará uma DNE $\Omega^{1}$ do segundo bloco, cuja ordem é

$$
\left(\delta-\delta_{0}\right) v_{0}+\ln \left(\Omega_{1}\right)=v_{2}+v_{1}-\left(m_{1}-\delta_{r}-1\right) v_{0}-1
$$

- $G^{1}=x^{\delta-\delta_{0}} \Omega_{1}$.

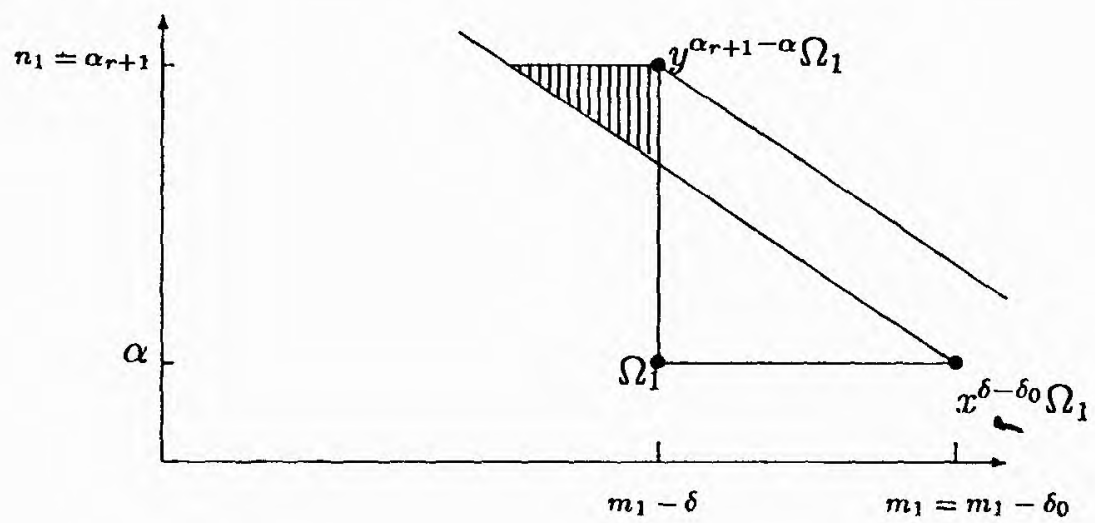


Temos que $\alpha_{1}=\alpha$ e $\delta_{1}=\delta$. Além disso, no segundo bloco temos uma DNE $\Omega^{1}$ obtida pela redução final, módulo $\left\{d x, d y, \Omega_{1}\right\}$ do $S$-processo envolvendo $F^{1} \Omega_{1}$, cuja ordem é

$$
\left(\alpha_{r+1}-\alpha\right) v_{1}+\ln \left(\Omega_{1}\right)=v_{2}+\left(n_{1}-\alpha_{1}+1\right) v_{1}-\left(m_{1}-1\right) v_{0}-1
$$

o que conclui o resultado para $i=1$.

Vamos supor que tenhamos construído as DNEM's do primeiro bloco $\Omega_{1}, \ldots, \Omega_{k+s}$, com $v\left(\Omega_{k+s-1}\right)+1=\alpha v_{1}-\delta v_{0}$. Consequientemente, temos no segundo bloco $\Omega^{1}, \ldots, \Omega^{k+s-1}$.

Situemos $\alpha_{k}<\alpha<\alpha_{r+1-s}$ e $\delta_{k}<\delta<\delta_{r+1-s}$, com $k$ e $s$ máximos.

Dois casos podem ocorrer:

1. $v\left(F^{k+s-1}\right)=\left(\alpha_{r-s+1}-\alpha\right) v_{1}$ e $v\left(G^{k+s-1}\right)=\left(\delta-\delta_{k}\right) v_{0}$.

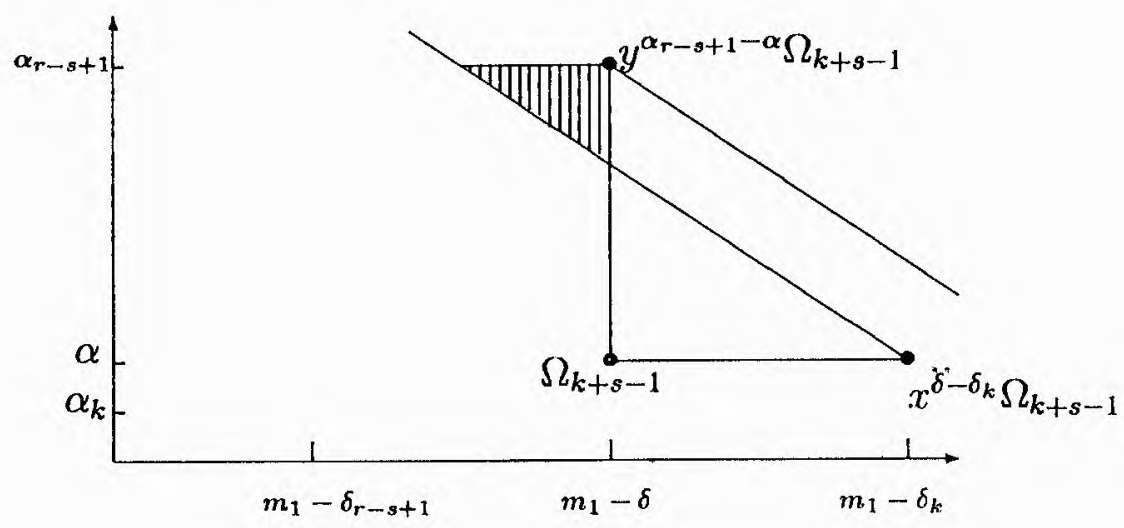

Neste caso, $\alpha \doteq \alpha_{k+1}, \delta=\delta_{k+1}, \Omega_{k+s}$ é obtida por uma redução final de um $S$-processo que envolve a parcela $x^{\delta-\delta_{k}} \Omega_{k+s-1}$ e, por hipótese de indução, temos

$$
v\left(\Omega^{k+s-1}\right)+1=v_{2}+\left(n_{1}-\alpha_{k+1}+1\right) v_{1}-\left(m_{1}-\delta_{k}-1\right) v_{0} .
$$

A análise agora se desdobra em dois subcasos.

(a) Se não existir uma nova DNEM no primeiro bloco, então $v\left(\Omega_{k+s}\right)+$ $1=\bar{\alpha} v_{1}-\bar{\delta} v_{0}$ é tal que $\alpha_{k+2}=\alpha_{r-s}=\bar{\alpha}, \delta_{k+2}=\delta_{r-s}=\bar{\delta} \mathrm{e}$ as reduções finais dos dois $S$-processos que envolvem $F^{k+s} \Omega_{k+s}$ e 
$G^{k+s} \Omega_{k+s}$ originam DNE's no segundo bloco cujas ordens, pelo Lema A.1 e para Observação A.1, são:

$$
\begin{gathered}
\left.\begin{array}{l}
\ln \left(F^{k+s} \Omega_{k+s}\right) \\
\ln \left(G^{k+s} \Omega_{k+s}\right)
\end{array}\right\}=v\left(\Omega^{k+s-1}\right)+v\left(G^{k+s-1}\right)-v\left(F^{k+s-1}\right)+\left\{\begin{array}{l}
v\left(F^{k+s}\right) \\
v\left(G^{k+s}\right)
\end{array}=\right. \\
=v_{2}+\left(n_{1}-\alpha_{k+1}+1\right) v_{1}-\left(m_{1}-\delta_{k}-1\right) v_{0}+\left(\delta_{k+1}-\delta_{k}\right) v_{0}- \\
-\left(\alpha_{r-s+1}-\alpha_{k+1}\right) v_{1}+\left\{\begin{array}{l}
v\left(F^{k+s}\right) \\
v\left(G^{k+s}\right)-1
\end{array}\right. \\
=v_{2}+\left(n_{1}-\alpha_{r-s+1}+1\right) v_{1}-\left(m_{1}-\delta_{k+1}-1\right) v_{0}+\left\{\begin{array}{l}
v\left(F^{k+s}\right) \\
v\left(G^{k+s}\right)
\end{array}-1\right.
\end{gathered}
$$

Como $\left\{v\left(F^{k+s}\right), v\left(G^{k+s}\right)\right\}=\left\{\left(\alpha_{r-s+1}-\alpha_{r-s}\right) v_{1},\left(\delta_{r-s}-\delta_{k+1}\right) v_{0}\right\}$ temos que as ordens acima são

$$
v_{2}+\left(n_{1}-\alpha_{r-s}+1\right) v_{1}-\left(m_{1}-\delta_{k+1}-1\right) v_{0}-1
$$

e

$$
v_{2}+\left(n_{1}-\alpha_{r-s+1}+1\right) v_{1}-\left(m_{1}-\delta_{r-s}-1\right) v_{0}-1 .
$$

Como $k+2=r-s$ o resultado segue neste subcaso.

(b) Se existir uma nova DNEM no primeiro bloco, então teremos $v\left(\dot{\Omega}_{k+s}\right)+1=\bar{\alpha} v_{1}-\bar{\delta} v_{0}$, com duas situações podendo ocorrer.

i. $v\left(F^{k+s}\right)=\left(\alpha_{r-s+1}-\bar{\alpha}\right) v_{1}$ e $v\left(G^{k+s}\right)=(\bar{\delta}-\delta) v_{0}$. Neste caso $\bar{\alpha}=\alpha_{k+2}$ e $\bar{\delta}=\delta_{k+2}$.

Temos; então que a redução final do $S$-processo envolvendo $F^{k+s} \Omega_{k+s}$ origina $\Omega^{k+s}$, cuja ordem pelo Lema A.1 e Observação A.1 é

$$
\begin{aligned}
v\left(\Omega^{k+s}\right) & =v\left(\Omega^{k+s-1}\right)+v\left(F^{k+s}\right)-v\left(F^{k+s-1}\right)+v\left(G^{k+s-1}\right)= \\
& =v_{2}+\left(n_{1}-\alpha_{k+2}-1\right) v_{1}-\left(m_{1}-\delta_{k+1}-1\right) v_{0}-1
\end{aligned}
$$

e o resutado segue na presente situação. 
ii. $v\left(F^{k+s}\right)=(\bar{\delta}-\delta) v_{0}$ e $v\left(G^{k+s}\right)=\left(\alpha_{r-s+1}-\bar{\alpha}\right) v_{1}$. Neste caso $\bar{\alpha}=\alpha_{r-s}$ e $\bar{\delta}=\delta_{r-s}$.

Temos, então que

$$
v\left(\Omega^{k+s}\right)=v_{2}+\left(n_{1}-\alpha_{r-s+1}+1\right) v_{1}-\left(m_{1}-\delta_{r-s}-1\right) v_{0}-1 .
$$

Concluindo assim a prova no Caso 1).

2. $v\left(F^{k+s-1}\right)=\left(\delta-\delta_{k}\right) v_{0}$ e $v\left(G^{k+s-1}\right)=\left(\alpha_{r-s+1}-\alpha\right) v_{1}$.

A demonstração deste caso é totalmente análoga ao Caso 1) e será omitida.

A proposição anterior nos diz que se $\left\{\Omega_{1}, \ldots, \Omega_{r}\right\}$ é o coujunto das DNEM's do primeiro bloco e $\left\{v\left(\Omega_{i}\right)+1 ; i=1, \ldots, r\right\}=\left\{\alpha_{i} v_{1}-\delta_{i} v_{0} ; i=\right.$ $1, \ldots, r\}$, então existem $r+1 \mathrm{DNE}$ 's $\Omega^{i}$ no segundo bloco, tal que o conjunto $\left\{v\left(\Omega^{i}\right)+1 ; i=0, \ldots, r\right\}$ é igual a

$$
\left\{v_{2}+\left(n_{1}-\alpha_{i+1}+1\right) v_{1}-\left(m_{1}-\delta_{i}-1\right) v_{0} ; i=0, \ldots, r\right\},
$$

$\operatorname{com} \delta_{0}=0, \alpha_{0}=1, \alpha_{r+1}=n_{1}$ e $\delta_{r+1}=m_{1}-1$.

Observação A.2 Da Proposição A.1 se deduz que as ordens de toda DNE decrita é menor do que $v_{2}-v_{0}-1$.

De fato a afirmação é óbvia no caso de DNE's do primeiro bloco.

Por outro lado se $\Omega$ é $D N E$ do segundo bloco com $v(\Omega) \geq v_{2}-v_{0}-1$, então para algum $i \geq 0, v(\Omega)=v_{2}+\left(n_{1}-\alpha_{i+1}+1\right) v_{1}-\left(m_{1}-\delta_{i}-1\right) v_{0}-1 \geq v_{2}-v_{0}-1$.

Como $n_{1} v_{1}=m_{1} v_{0}$, temos que a desigualdade acima se escreve como $v_{1}+v_{0} \geq \alpha_{i+1} v_{1}-\delta_{i} v_{0}-v_{0}$. Desse modo teríamos

$$
\begin{aligned}
v_{1}+v_{0} & \geq \alpha_{i+1} v_{1}-\delta_{i} v_{0}-v_{0} \geq \alpha_{i} v_{1}-\delta_{i} v_{0}+v_{1}-v_{0} \geq \\
& \geq \alpha_{i} v_{1}-\delta_{i} v_{0}+1>\alpha_{i} v_{1}-\delta_{i} v_{0}=v\left(\Omega_{k}\right)+1,
\end{aligned}
$$

para alguma $D N E M \Omega_{k}$ do primeiro bloco, o que é um absurdo!

Em particular, temos que a lacuna limitante é menor ou igual a $v_{2}-v_{0}$.

Vamos provar que não existe $g \in \mathcal{O}$, tal que $v\left(g \Omega^{i}\right)=v\left(\Omega^{j}\right)$ para $\Omega^{i}$ e $\Omega^{j}$ DNE's do segundo bloco como descritas anteriormente. 
Lema A.2 Com as notações da Proposição A.1 temos que se a,b,c $\in \mathbb{N}$ então

$$
\begin{aligned}
a v_{0}+b v_{1}+c v_{2}+v_{2}+ & \left(n_{1}-\alpha_{i+1}+1\right) v_{1}-\left(m_{1}-\delta_{i}-1\right) v_{0}= \\
& =v_{2}+\left(n_{1}-\alpha_{j+1}+1\right) v_{1}-\left(m_{1}-\delta_{j}-1\right) v_{0}
\end{aligned}
$$

se, e somente se, $a=b=c=0$ e $i=j$.

Dem.: Se $c \neq 0$ ou $a \geq m_{1}$ ou $b \geq n_{1}$, então teríamos que

$$
v_{2}+\left(n_{1}-\alpha_{j+1}+1\right) v_{1}-\left(m_{1}-\delta_{j}-1\right) v_{0}>v_{2}
$$

o que pela observação anterior não pode ocorrer.

Consideremos então $c=0, a<m_{1}$ e $b<n_{1}$.

$\mathrm{A}$ igualdade do enunciado é equivalente a $a v_{0}+b v_{1}-\alpha_{i+1} v_{1}+\delta_{i} v_{0}=$ $-\alpha_{j+1} v_{1}+\delta_{j} v_{0}$, ou ainda,

$$
\left(b-\alpha_{i+1}+\alpha_{j+1}\right) m_{1}=\left(\delta_{j}-\delta_{i}-a\right) n_{1} .
$$

Como $\left(n_{1}, m_{1}\right)=i$, temos que $b-\alpha_{i+1}+\alpha_{j+1}=k n_{1}$ e $\delta_{j}-\delta_{i}-a=k m_{1}$, $\operatorname{com} k \in \mathbb{Z}$.

Se $k>0$, então $\delta_{j}=a+\delta_{i}+k m_{1}>m_{1}$, o que é uma contradição.

Se $k<0$, então $\alpha_{i+1}=\alpha_{j+1}+b-k n_{1}>n_{1}$ que também é uma contradição.

Segue que $k=0$, -i.e., $\alpha_{i+1}=\alpha_{j+1}+b$ e $\delta_{j}=\delta_{i}+a$. A primeira igualdade implica que $i \leq j$, enquanto que a segunda $j \leq i$, o que permite concluir que $i=j$ e $a=b=0$.

O lema anterior garante que o diagrama de lacunas especiais de uma curva com semigrupo $\Gamma=\left\langle 2 n_{1}, 2 m_{1}, v_{2}\right\rangle$ tem como primeiro bloco

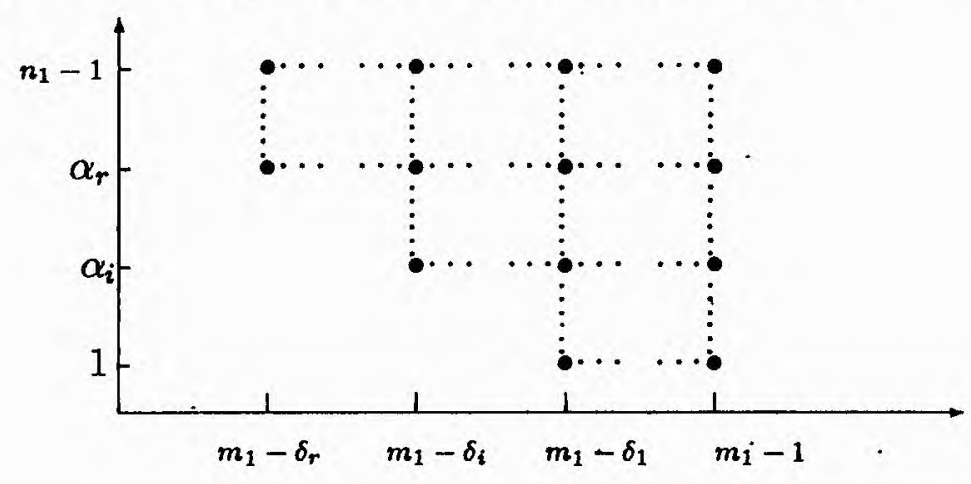


e o segundo bloco contém pelo menos os pontos

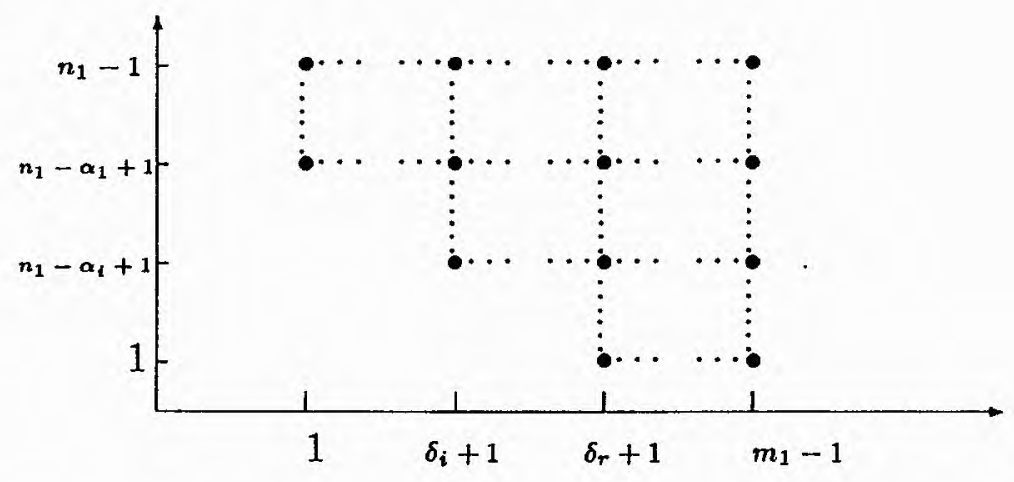

O número de pontos no primeiro bloco é

$$
A=\sum_{i=1}^{r}\left(\delta_{i}-\delta_{i-1}\right)\left(n_{1}-\alpha_{i}\right)
$$

pontos e no segundo bloco pelo menos

$$
B=\sum_{i=0}^{r}\left(\delta_{i+1}-\delta_{i}\right)\left(\alpha_{i+1}-1\right)
$$

onde $\delta_{0}=0$.

Deste modo, ao todo, teremos no diagrama de lacunas especiais pelo menos $A+B=\left(n_{1}-1\right)\left(m_{1}-1\right)$ pontos.

Como pelo resultado de Laudal e Pfister,

$$
\sharp(\Lambda \backslash \Gamma)=\left(n_{1}-1\right)\left(m_{1}-1\right),
$$

temos que as DNE's do segundo bloco, determinadas na Proposição A.1 são na verdade todas as DNEM's do segundo bloco.

Observe que se tomarmos as duas figuras anteriores e girarmos $180^{\circ}$ uma delas, então poderemos encaixá-las de modo a obter um retângulo com área $\left(n_{1}-1\right)\left(m_{1}-1\right)$. Portanto, as figuras, ou melhor, os blocos que compõem o diagrama de lacunas especiais para ramos com semigrupo $\left\langle 2 n_{1}, 2 m_{1}, v_{2}\right\rangle$ se determinam mutuamente.

Reunindo os resultados obtidos anteriormente, temos o seguinte teorema. 
Teorema A.1 Seja $C$ um ramo com semigrupo $\Gamma=\left\langle 2 n_{1}, 2 m_{1}, v_{2}\right\rangle$. Defina $\Omega_{-1}=d x, \Omega_{0}=d y$ e $\Omega^{0}=d h_{2}, c o m h_{2}$ como dado na Proposição 2.1. Então uma Base Standard Mínima para $\mathcal{O} d \mathcal{O}$ pode ser dada por

$$
\left\{\Omega_{-1}, \Omega_{0}, \Omega_{1}, \ldots, \Omega_{r}\right\} \cup\left\{\Omega^{0}, \Omega^{1}, \ldots, \Omega^{r+1}\right\},
$$

onde cada $\Omega_{i+1}$ para $i=0, \ldots, r-1$ é uma DNEM do primeiro bloco dada pela Proposição 3.3, e cada $\Omega^{i+1}$ para $i=0, \ldots r$ é uma $D N E M$ do segundo bloco, dada pela Proposição A.1.

Além disso, se $\left\{v\left(\Omega_{i}\right)+1 ; i=1, \ldots, r\right\}=\left\{\alpha_{i} v_{1}-\delta_{i} v_{0} ; i=1, \ldots, r\right\}$, então $\left\{v\left(\Omega^{i+1}\right)+1 ; i=0, \ldots, r\right\}=\left\{v_{2}+\left(n_{1}-\alpha_{i+1}-1\right) v_{1}-\left(m_{1}-\delta_{i}-1\right) v_{0} ; i=\right.$ $0, \ldots, r\}$, onde $\alpha_{r+1}=n_{1}$ e $\delta_{0}=0$.

Note que se não tivermos DNEM no primeiro bloco, então $r=0$ e $\lambda=\beta_{2}$. Assim, no segundo bloco temos apenas uma DNEM $\Omega^{1}$, com $v\left(\Omega^{1}\right)+1=$ $v_{2}+v_{1}-\left(m_{1}-1\right) v_{0}=\beta_{2}+v_{0}$, como já havíamos observado no ínicio desta seção.

Como já tivemos á oportunidade de observar, o invariante $\Lambda \backslash \Gamma$ é muito mais fino do que o invariante $\lambda$ de Zariski e o número $\tau$ de Tjurina, mesmo que considerados simultaneamente, i.e., podemos ter ramos equisingulares que possuem mesmo invariante $\lambda$ de Zariski, mesmo número $\tau$ de Tjurina, porém com conjuntos de lacunas especiais distintos, como mostra o seguinte exemplo.

Exemplo A.1 Considere os ramos da forma

$$
\left\{\begin{array}{l}
x=t^{8} \\
y=t^{18}+t^{20}+a t^{22}+b t^{23}+\cdots
\end{array}\right.
$$

com $a \in K e b \in K^{*}$.

Utilizando a Proposição 2.1, vemos que o semigrupo de valores dos ramos da forma acima é $\Gamma=\langle 8,18,77\rangle$, cujo condutor é $\mu=124$.

Note que $20 \notin \Gamma \cup\left(\Gamma+v_{1}-v_{0}\right)$, ou seja, o invariante de Zariski é $\lambda=20$ para todos os ramos como acima.

Uma vez que $n_{1}=4, n_{2}=2$ e $m_{1}=9$, temos que

$$
\mu-\tau=\left(n_{1}-1\right)\left(m_{1}-1\right)=24,
$$

ou seja, todos os ramos descritos acima possuem os mesmos valores para os invariantes $\lambda e \tau$. 
Vejamos o conjunto de lacunas especiais $\Lambda \backslash \Gamma$.

Pelo teorema-anterior, basta encontrarmos as ordens das DNEM's do primeiro bloco. Para tanto, aplicamos a Proposição 3.3.

A saber, temos

$$
\begin{gathered}
\Omega_{1}=x d y-\frac{9}{4} y d x=\left(2 t^{27}+4 a t^{29}+5 b t^{30}+\cdots\right) d t \\
\Omega_{2}=9 x \Omega_{1}-y d y=\left((36 a-38) t^{37}+45 b t^{38}+\cdots\right) d t .
\end{gathered}
$$

Se $a \neq \frac{19}{18}$, então as DNEM's do primeiro bloco são tais que $v\left(\Omega_{1}\right)+1=28$ e $v\left(\Omega_{2}\right)+1=38$.

Se $a=\frac{19}{18}$, então a única DNEM do primeiro bloco é $\Omega_{1}, \operatorname{com} v\left(\Omega_{1}\right)+1=$ 28.

Desse modo, pelo teorema anterior temos:

\begin{tabular}{|c|c|}
\hline condição & $\Lambda \backslash \Gamma$ \\
\hline$a \neq \frac{19}{18}$ & $28,38,46,47,55,57,63,65,67,71,73,75$ \\
& $79,81,83,87,89,91,97,99,105,107,115,123$ \\
\hline$a=\frac{19}{18}$ & $28,39,46,47,55,57,63,65,67,71,73,75$ \\
& $79,81,83,87,89,91,97,99,105,107,115,123$ \\
\hline
\end{tabular}




\section{Bibliografia}

[An] ANGERmüller, G., Die Wertehalbgruppe einer Ebenen Irreduzibien Algebroiden Kurve, Math. Zeitschr. 153, 267-282 (1977).

[AM] AbhyankaR, S.S. AND Moh, T., Newton-Puiseux Expansion and Generalized Tschirnhausen Transformation, J. Reine Angew. Math. 260, 47-83 (1973) and 261, 29-54 (1973).

[Az] Azevedo, A., The Jacobian Ideal of a Plane Algebroid Curve, Ph. D. Thesis, Purdue University (1967).

[Bec1] BECKer, T., Standard Bases and Some computations in Rings of Power Series, J. Symbolic Computation 10, 165-178 (1990).

[Bec2] Becker, T., Standard Bases in Power Series Rings: Uniqueness and Superfluous Critical Pairs, J. Symbolic Computation 15, 251-265 (1993).

[Ber] BERGER, R.W., Differentialmoduln Eindimensionaler Lokaler Ringe, Math. Z. 81, 326-354 (1963).

[BGa] Bruce, J.W. and Gaffney, T.J., Simple Singularities of Mappings $\mathbb{C}, 0 \longrightarrow \mathbb{C}^{\mathfrak{Q}}, 0$, J. London Math. Soc. (2) 26, 465-474 (1982).

[BGM] Briançon, J.; Granger, M. and Maisonobe, Ph. Le Nombre de. Modules du Germe de Curbe Plane $X^{a}+Y^{b}=0$, Math. Ann. 279, 535-551 (1988).

[Car] Carbonne, P., Sur le Différentielles de Torsion, Journal of Algebra 202, 367-403 (1998).

[CF] Clausen, M. and Fortenbacher, A., Efficient Solution of Linear Diophantine Equations, J. Symbolic Computation 8, 201-216 (1989). 
[D1] Delorme, C., Sous-Monoides d'Intersection Complète de $\mathbb{N}$, Anu. Sci. École Norm. Sup. 9, 145-154 (1976).

[D2] Delorme, C., Sur les Modules des Singularités de Courbes Planes, Bull. Soc. Math. France 106, 417-446 (1978).

[E] EBEY, S., The Classification of Singular Points of Algebraic Curves, Trans. amer. Math. Soc. 118, 454-471 (1965).

[He] Heinrich, J., On a Conjeture of Azevedo, Arch. Math. Vol. 64, 188-198 (1995).

[HK] Herzog, J. and Kunz, E., Die Wertihalbgruppe eines Lokalen Rings der Dimension 1, Ber. Heidelberg Akad. Wiss. 2, 1-46 (1971).

[LaP] Laudal, O. A. ANd Pfister, G., Local Moduli and Singularities, Lecture Notes in Mathematics, 1310.

[LuP] Luengo, I. And Pfister, G., Normal Forms and Moduli Spaces of Curve Singularities with Semigroup $\langle 2 p, 2 q, 2 p q+d\rangle$, Compositio Mathematica 76, 247-264 (1990).

[M] Miller, J.L., Analogs of Groebner Bases in Polynomial Rings over a Ring, J. Symbolic Computation 21, 139-153 (1996).

[Pe1] PeraIre, R., Tjurina Number of a Generic Irreducible Curve Singularity, Journal of Algebra 196, 114-157 (1997).

[Pe2] Peraire, R., Moduli of Plane Curve Singularities with a Single Characteristic Exponent, Proceedings of the American Mathematical Society 126 (1), 25-34 (1998).

[Pi] Pinkham, H. C., Deformations of Algebraic Varieties with $G_{m}$-action, Astérisque 20, Sociéte Mathématique de France (1974).

[RS] Robbiano, L. And Sweedler, M., Subalgebra bases, Proc. Commutative Algebra Salvador, Lectures Notes in Math. Vol 1430, 61-87.

[Ru] Ruiz, J. M., The Basic Theory of Power Series, Advanced Lectures in Mathematics, Vieweg (1993).

[T] TeIssier, B., Appendice in. [Z2]. 
[Z1] ZARISKI, O., Characterization of Plane Algebroid Curves whose Module of Differentials has Maximum Torsion, Proceedings Nat. Acad. of Science U.S.A. 56, 781-786 (1966) .

[Z2] Zariski, O., Le Problème des Modules pour les Branches Planes, Cours donné au Centre de Mathématiques de L'École Polytechinique (1973). 


\section{Símbolos e Notações}

$K[[\underline{X}]]$
$\underline{X}^{\alpha}$
$\mathbb{T}$
$\preceq$
$D^{*}$
$\operatorname{car}(K)$
$\operatorname{deg}_{\rho}(f)$
$\mathcal{M}_{\underline{X}}$
$\mathcal{M}$
$h t(f)$
$F^{\alpha}$
$\left\langle h_{0}, \ldots, h_{r}\right\rangle$
$v_{0}, v_{1}, \ldots, v_{g}$
$S(f, g)$
$\mathcal{O}$
$\overline{\mathcal{O}}$
$v(g)$
$\Gamma$
$\mu$
$I(f, g)$
$R Y(f, g)$

$K\left[\left[X_{1}, \ldots, X_{n}\right]\right]$

$\prod_{i=1}^{n} X_{i}^{\alpha_{i}}$

$\left\{\underline{X}^{\alpha} ; \alpha \in \mathbb{N}^{n}\right\}$

ordem monomial

$D \backslash\{0\}$

característica do corpo $K$

grau pesado de $f$ com respeito à $\rho$

ideal maximal de $K[[\underline{X}]]$

ideal maximal de $K[[t]]$

altura de $F$

$\prod_{i=1}^{\sharp F} f_{i}^{\alpha_{i}} \operatorname{com} f_{i} \in F$

semigrupo (ideal) gerado pelos(as) inteiros (séries) $h_{0}, \ldots, h_{r}$ sistema mínimo de geradores de geradores de $1 \mathrm{~m}$ semigrupo

$S$-processo de $f$ e $g$

anel local

fecho inteiro de $\mathcal{O}$

valorização de $\mathcal{O}$ calculada em $g$

semigrupo

condutor de um semigrupo

multiplicidade de interseção de $g \operatorname{com} f$

resultante em $Y$ de $f$ e $g$ 


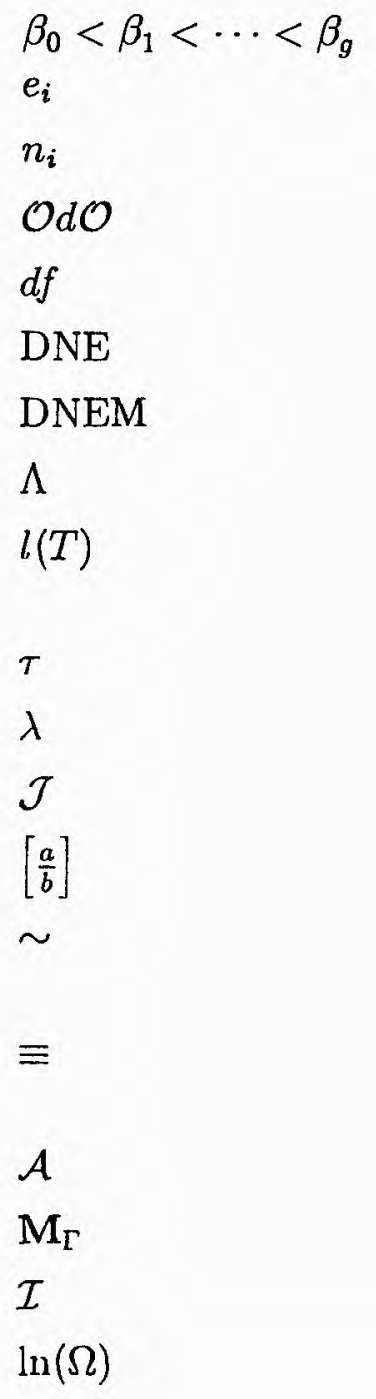

seqüência característica

$\operatorname{MDC}\left(\beta_{0}, \ldots, \beta_{i}\right)=\operatorname{MDC}\left(v_{0}, \ldots, v_{i}\right)$

$\frac{e_{i-1}}{e_{i}}$

$\mathcal{O}$-módulo de diferenciais

diferencial de $f$

Diferencial Não Exata

Diferencial Não Exata Minimal

$\{v(\Omega)+1 ; \Omega \in \mathcal{O} d \mathcal{O}\}$

comprimento do submódulo de torção cle $\mathcal{O} d \mathcal{O}$

número de Tjurina

invariante de Zariski

ideal jacobiano

parte inteira da fração $\frac{a}{b}$

equivalência de curvas algebróides irredutíveis

equisingularidade de curvas

algebróides irredutíveis

anel de Puiseux

Classe de equisingularidade

ideal $\left\langle f, f_{x}, f_{y}\right\rangle$

menor expoente $l$ de $t$ em $\Omega$

tal que $\operatorname{MDC}\left(v_{0}, v_{1}\right) \times(l+1)$ 


\section{Índice Remissivo}

A

Abhyankar-Moh, método de 37 álgebras, Base Standard de 5 altura de uma série 4 amplitude 4

analítica, equivalência xiii, 60 anéis de Puiseux 26, 64

Apéry, sequiência de 67 aproximação de raízes 37 Azevedo, conjectura de 76 Azevedo-Zariski, método de 36

B

Base Standard 1

de álgebras 5,6

de módılos 17

Mínima 8, 22

Reduzida 16, 22

bloco, diferencial de $i$-ésimo 77

C

canônica de Zariski, parametrização 170

canônico, ramo 76

classe de equisingularidade 66

codimensão do ideal jacobiano 53

coeficiente líder 3

completa, interseção 35

condutor de um semigrupo 27

conjectura de Azevedo 76

contraexemplo de Heinrich 76 coordenadas, mudanças de 65

curva

algebóide irredutível 25

gênero de uma 26

semigrupo de uma 26

curvas, equivalência de 59

D

Delorme, fórmula de 109

diagrama de lacunas 71

especiais 69

Dickson, lema de 2

diferencial, de $i$-ésimo bloco 77

exata 44

não exata 44

ordem de uma 43

diferenciais,

módulo de 42

não exatas minimais 45

$\mathbf{E}$

equidiferenciabilidade 50

equisingularidade 34

classe de, 66

equivalência

analítica xiii, 60

de curvas 59

topológica xiii, 60 
escrita

privilegiada 69

única 69

especiais,

diagrama de lacunas 69 lacunas 46

exata, diferencial 44

F

formas normais 149

fórmula de Delorme 109

G

genérica, curva 71

gênero de

um semigrupo 26

uma curva 26

Gorenstein 35

grau 2

pesado 3

$\mathbf{H}$

Heinrich, contraexemplo de 76

homotetias 65

I

ideal jacobiano, 53, 104

codimensão do 53

multiplidade do 53

interseção completa 35

invariante de Zariski 68

$\mathbf{J}$

jacobiano, ideal 53, 104

$\mathbf{L}$

$\lambda$-canônico, ramo 121

lacuna 67

limitante 44 lacunas especiais, 46 diagrama de 69

M

método de

Abhyankar-Moh 37

Azevedo-Zariski 36

Mínima, Base Standard 8, 22

módulo de diferenciais 42

Módulos, Base Standard de 17

Moh, método de Abhyankar- 37

mudanças de coordenadas 65

multiplicidade

de uma curva 26

do ideal jacobiano 53

$\mathbf{N}$

normais, formas 149

número de Tjurina 53

$\mathbf{O}$

ordem

de uma diferencial 43

lexicográfica 2

graduada 2

monomial 1

pesada 3

$\mathbf{P}$

parametrização 26

canônica de Zariski 170

primitiva 26

potência líder 3

primitiva, parametrização 26

privilegiada, escrita 69

produto de potências 4

Puiseux

anéis de 26,64

parametrização de 26 
$\mathbf{R}$

raízes, aproximação de 37

ramo 36,59

$\lambda$-canônico 121

canônico 76

redução

completa 5,18

final 5,18

reduzida, Base Standard 16, 22

$\mathrm{S}$

$S$-processo 8, 19

mínimos 11, 19

em uma direção 81, 183

SAGBI 1

semigrupo, 27

condutor de um 26

gênero de um 26

seqüência

característica 36

de Apéry 67

SG-Base 17

sistema mínimo de geradores 14

submódulo de torção 43

$\mathrm{T}$

termo líder 3

Tjurina, número de 53

topológica, equivalência xiii, 60

torção, submódulo de 43

$\mathrm{U}$

única, escrita 69

$\mathbf{Z}$

Zariski, invariante de 68 método de Azevedo- 36

paramet. canônica de 170 


\section{ERRATA}

Além dos enganos ortográficos, os quais não prejudicam o entendimento dos resultados apresentados no trabalho, algumas observações e correções se fazem necessárias.

Na página 15 antes do Teorema 1.3, devemos observar que a definição de Base Standard, bem como os resultados: Proposição 1.2, Teorema 1.1, Lema 1.3 e o Teorema 1.2, permanecem verdadeiros considerando $F$ um conjunto infinito e realizando as devidas modificações.

Deste modo, verifica-se que o Teorema 1:3, sempre fornece uma Base Standard para uma subálgebra finitamente gerada e finaliza em um número finito de passos se a subálgebra possuir uma Base Standard finita.

Na página 22, como no caso de subálgebras, uma observação similar a feita acima deve ser feita antes do Teorema 1.5.

Na página 43, na demonstração da Proposição 2.2, onde se lê "é equivalente a dizer", leia-se "é consequiência do fato".

Na página 56, na demonstração do Corolário 2.1, onde se lê "Pelo teorema anterior", leia-se "Pela proposição anterior".

Na página 64, a Proposição 3.2, deve ser considerada no contexto das curvas algebróides irredutíveis planas (ramos).

São Carlos, abril de 2001.

Marcelo Escudeiro Hernandes mehernandes@uem.br 